\title{
Accelerated Aging Studies and Environmental Stability of Prototype Tamper Tapes
}
B. W. Wright
C. W. Wright
A. R. Bunk
B. A. Metz
G. E. Pickett

May 1995

Prepared for the U.S. Department of Energy. Office of Research and Development (NN-20) under Contract DE-AC06-76RLO 1830

Pacific Northwest Laboratory Operated for the U.S. Department of Energy by Battelle Memorial Institute

\section{\%Battelle}




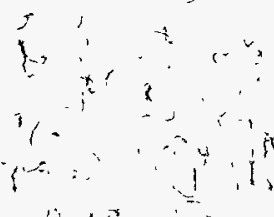

'

$\because !$

$1 \%$

C'sir

DISCLAIMER.

This report was prepared as an account of work sponsored by an agency of the

United States Government. Neither thie United States Government nor any agency thereof, nor Battelle Memorial Institute, nor any of their employees, makes any ( wàranty, expressed or implied, or assumes any legal liability or responsibility for the accuracy, complèteness, or usefulness of any information, apparatus, product, or process disclosed, or represents that its use would not infringe privately owned rights. Reference herein to any specific commercial product, process, or service by trade name, trademark; manúfacturer, or otherwise dóes nòt necessarily constitute or imply its endorsement, recommendation, or favoring by the United States Government or any agency thereof, or Battelle Mémorial Institute. The viéws and opinions of authors expressed herein do not necessarily state or reflect those of the

\section{United States Government or any ágency thêreof.}

\section{PACIFIĆ NOORTHWEST LABOORATÓRY operated by \\ BATTELLEMEMÓRIALLINSTITUUTTE for, the \\ UNITED STATES DEPARTMENT ÓF ENERGY} under Contract DE-ACO6-76RLO 1830

$$
\text { Printed in the United States of Ámerica }
$$

Available to DOE and DOE contractors from the Office of Scientific and Technical Information, P.O. Box 62,-Oak Ridge, TN 37831; prices availabléfrom (615) 576-8401. FTS 626-8401.

Available to the public from the National Technical Information Service, U.S. Department of Commerce, 5285 Port Royal Rd., Springfield, VA 22161. 
ACCELERATED AGING STUDIES AND ENVIRONMENTAL STABLITY OF PROTOTYPE TAMPER TAPES

B. W. Wright

C. W. Wright

A. R. Bunk*

B. A. Metz*

G. E. Pickett*

May 1995

Prepared for

the U.S. Department of Energy

Office of Research and Development (NN-20)

under Contract DE-AC06-76RLO 1830

Pacific Northwest Laboratory

Richland, Washington 99352

* Battelle Columbus Laboratory

Columbus, Ohio

FI DISTRIBUTION OF THIS DOCIMMENT IS UNLIMITED 


\section{DISCLAIMER}

Portions of this document may be illegible in electronic image products. Images are produced from the best available original document. 


\section{ABSTRACT}

This report describes the results of accelerated aging experiments (weathering) conducted on prototype tamper tapes bonded to a variety of surface materials. The prototype tamper tapes were based on the patented Confirm ${ }^{\circledR}$ tamper-indicating technology developed and produced by $3 \mathrm{M}$ Company. Tamper tapes bonded to surfaces using pressure sensitive adhesive (PSA) and four rapid-set adhesives were evaluated. The configurations of the PSA-bonded tamper tapes were 1.27-cm-wide Confirm ${ }^{\circledR} 1700$ windows with vinyl underlay and 2.54-cm-wide Confirm ${ }^{\circledR} 1700$ windows with vinyl and polyester underlays. The configurations of the rapid-set adhesive-bonded tamper tapes were $2.54-\mathrm{cm}$-wide Confirm ${ }^{\circledR}(1700,1500$ with and without primer, and 1300) windows with vinyl underlay. Surfaces used for bonding included aluminum, steel, stainless steel, $\operatorname{Kevlar}^{\circledR}$, brass, copper, fiberglass/resin with and without gel coat, polyurethane-painted steel, acrylonitrile:butadiene:styrene plastic, polyester fiberglass board, Lexan polycarbonate, and cedar wood. Weathering conditions included a QUV cabinet (ultraviolet light at $60^{\circ} \mathrm{C}$, condensing humidity at $40^{\circ} \mathrm{C}$ ), a thermal cycling cabinet $\left(-18^{\circ} \mathrm{C}\right.$ to $46^{\circ} \mathrm{C}$ ), a Weather-O-Meter (Xenon lamp), and exposure outdoors in Daytona Beach, Florida. Environmental aging exposures lasted from 7 weeks to 5 months. After exposure, the tamper tapes were visually examined and tested for transfer resistance. Tamper tapes were also exposed to a variety of chemical liquids (including organic solvents, acids, bases, and oxidizing liquids) to determine chemical resistance and to sand to determine abrasion resistance. 



\section{EXECUTIVE SUMMARY}

A tamper tape is an adhesive-backed label that possesses various tamper-indicating, transferresistant, or counterfeit-resistant properties. Ongoing research has been conducted to develop tamper tapes that combine the best features of commercially-available tamper tapes with state-ofthe-art design, unique identification, and counterfeit-resistant features. As part of this development, numerous prototype tamper tapes adhered to different surfaces were evaluated under various environmental aging conditions. The exposed tamper tapes were visually inspected and evaluated for transfer resistance to determine the effects of the weathering conditions.

The prototype tamper tapes are based on the patented Confirm ${ }^{\circledR}$ tamper-indicating technology developed and produced by 3M Company (Safety and Security Systems Division, 3M Center, St. Paul, Minnesota). Several interim prototype designs have been produced as more advanced features are developed. The complete tamper tape consists of a top layer of Confirm ${ }^{\circledR}$ bonded to an underlay material (vinyl or polyester) that provides support around three sides of the tamper tape to allow the fragile Confirm ${ }^{\circledR}$ to be efficiently applied to a surface. The area where the underlay is not present is known as the Confirm ${ }^{\circledR}$ window. The Confirm ${ }^{\circledR}$ is made of glass beads embedded in a brittle bonding material. If transfer is attempted, the logo pattern reflected from beneath the glass beads is distorted as the beads are disrupted from the bonding layer.

Weathering studies were performed using prototype tamper tapes applied to surfaces using both pressure sensitive adhesives (PSA) and reactive, rapid-set adhesives. Size configurations were approximately $5.1 \mathrm{~cm}$ by $10.2 \mathrm{~cm}$ with $1.27-\mathrm{cm}$ - and $2.54-\mathrm{cm}$-wide Confirm ${ }^{\circledR}$ windows. Surfaces for bonding included aluminum, steel, stainless steel, $\operatorname{Kevlar}{ }^{\circledR}$, brass, copper, fiberglass/resin with and without gel coat, acrylonitrile:butadiene:styrene (ABS) plastic, polyurethane-painted steel, polyester fiberglass board, Lexan polycarbonate, and cedar wood. Weathering conditions included a QUV cabinet (ultraviolet light at $60^{\circ} \mathrm{C}$ for 4 hours, condensing humidity at $40^{\circ} \mathrm{C}$ for 4 hours), a thermal cycling cabinet ( 12 hours at $-18^{\circ} \mathrm{C}$, followed by a 3 -hour warming to $46^{\circ} \mathrm{C}, 6$ hours at $46^{\circ} \mathrm{C}$, and, finally, a 3-hour cooling to $-18^{\circ} \mathrm{C}$ ), a Weather-O-Meter (Xenon lamp, continuous light, no water spray), and outdoor exposure at Daytona Beach, Florida (vertical south orientation). Environmental aging exposures lasted from 7 weeks to 5 months. After exposure, the tamper tapes were visually examined and tested for transfer resistance.

In initial studies, tamper tapes with PSA, 1.27-cm-wide Confirm ${ }^{\circledR} 1700$ windows, and a vinyl underlay were subjected to weathering in the QUV chamber, at Florida, and in the thermal cycling cabinet. Surfaces to which the tamper tapes were bonded included aluminum, steel, stainless steel, $\operatorname{Kevlar}^{\circledR}$, brass, copper, fiberglass/resin/gel coat, fiberglass/resin, and ABS plastic. 
QUV results were as follows: slight yellowing of the tamper tapes was noted after the first day or two of exposure; the steel panels rusted, resulting in staining of the tamper tapes; adhesion to the rough fiberglass/resin surface was poor; the security feature appeared to "dim" over time; and cracks appeared in the window areas near the conclusion of the test. Thermal cycling had no visual effects on the tamper tapes. After 7 weeks of exposure in Florida, the tamper tapes showed slight yellowing and some showed cracks in the upper corner. After 20 weeks, mildew was growing on some of the tamper tapes, and some tapes were beginning to "flake" in small areas and appeared to be losing adhesion to the surface.

Tamper tapes were evaluated for transfer resistance with and without exposure to weathering. Methods of tape removal included exposure to temperature extremes, sharp instruments, and chemicals/liquids. The results indicated that with care and patience, the tamper tapes (PSA, 1.27-cm-wide Confirm ${ }^{\circledR}$ window) could be removed using a razor blade and were, therefore, not secure against this threat.

The chemical compatibility of the Confirm ${ }^{\circledR}$ tamper tapes was investigated by applying tapes to aluminum plates and then soaking them in various chemical liquids. The tamper tapes were visually evaluated immediately after exposure and again after 24 hours. Organic solvents used were ethanol (no change), methylene chloride (adhesive softened), chloroform (adhesive softened), carbon tetrachloride (no change), hexane (discoloration after 24 hours), and dimethyl sulfoxide (printing discolored, adhesive softened). Acids included concentrated nitric acid (discoloration, ink destroyed, easily peeled from surface) and sulfuric acid (discoloration, ink degradation, easily scratched). Bases included two molar strengths of sodium hydroxide $(0.1 \mathrm{M}$, easily scratched, and $6 \mathrm{M}$, easily scratched and ink/printing destroyed). In addition, an oxidizing agent, hypochlorite (bleach, no change), was used.

In later studies, weathering studies were performed on tamper tapes with PSA, 2.54-cmwide Confirm ${ }^{\circledR} 1700$ windows, and both polyester and vinyl underlay. The tamper tapes with polyester underlay (12/93) were bonded to four surfaces, i.e., aluminum, steel, polyester fiberglass board, and a Military Specification (Mil. Spec.) polyurethane-painted steel. They were exposed to weathering conditions at Florida, in the Weather-O-Meter, in the QUV chamber, and in the thermal cycling cabinet. The tamper tapes with vinyl underlay were bonded to six surfaces, i.e., wood and Lexan polycarbonate in addition to those listed above, and were exposed to the same weathering conditions (with the exception of the Weather-O-Meter).

The evaluation/examination of tamper tapes after weathering was done by two or three persons who provided a descriptive commentary on the tamper tapes' appearance (with and without the $3 \mathrm{M}$ security illuminator) and evaluated the adhesion of the tamper tapes to the surfaces. 
After 138 days under the Weather-O-Meter conditions, the general appearance of the tamper tapes was fair to good, the security emblem appeared to be strong, and there was less than $2 \%$ debonding. After 5 months under the Florida exposure conditions, the general appearance of the tamper tapes was good to very good (except on steel where it was fair to good), the security emblem appeared to be strong in the window area, and there was less than $2 \%$ debonding. Under the QUV cabinet exposure conditions for 100 days, the general appearance of the tamper tapes was fair or fair to good, the security emblem was not visible (the area turned black), and greater than $25 \%$ debonding occurred on steel and aluminum. Few to no effects occurred from thermal cycling exposure for 126 days.

The transfer resistance of the $12 / 93$ prototype tamper tapes on four surfaces (roughened aluminum, roughened steel, fiberglass board, and polyurethane-painted steel) was evaluated after 14 days of exposure to QUV, thermal cycling, and control $\left(23^{\circ} \mathrm{C}, 50 \%\right.$ relative humidity) .conditions. All of the tapes could be removed from the test surfaces, although adhesive transfer occurred and residue remained on the roughened steel and polyurethane-painted steel surfaces.

The abrasion resistance of the 12/93 tamper tapes using sand was also evaluated. The evaluation indicated that the ink faded more and more in the bar code area after two passes of $2 \mathrm{~kg}$ each of sand. The ink was sufficiently removed after six passes of sand to prevent the bar code from being read with a reader. After three passes of sand, one-half of the security emblem was gone as determined with the security light. The entire emblem was destroyed after five passes of sand.

As development of the tamper tapes progressed, it was determined that increased security performance could be achieved by eliminating the PSA on the tamper tapes and substituting a more suitable reactive, rapid-set adhesive for surface bonding. Four candidate rapid-set adhesives (two epoxies, one polyurethane, and one commercial acrylic) were chosen for weathering evaluation. Two weathering studies using tamper tapes bonded to surfaces using the rapid-set adhesives were performed.

In the first study using rapid-set adhesives, tamper tapes with $2.54-\mathrm{cm}$-wide Confirm ${ }^{\circledR}$ windows were made in the laboratory out of Confirm ${ }^{\circledR} 1700$ and vinyl underlay materials bonded together using a PSA supplied by $3 \mathrm{M}$. Each of the four candidate rapid-set adhesives were used to bond these tamper tapes to six surfaces; i.e., roughened steel, roughened aluminum, cedar wood, polyester fiberglass board, Lexan polycarbonate, and Mil. Spec. polyurethane-painted steel. The tamper tapes were cured for several days and then were tested in the QUV cabinet, the thermal cycling cabinet, in a constant temperature room $\left(23^{\circ} \mathrm{C}, 50 \%\right.$ relative humidity), and at Florida. 
The tamper tapes were then visually examined to assess their appearance, the appearance of the security feature with the $3 \mathrm{M}$ security illuminator, and the adhesion of the tamper tapes to the surfaces. After 2 months of Florida exposure, neither of the epoxy adhesive-bonded tamper tapes showed loss of adhesion or changes to the appearance of their security features. The security features in the window area of the tamper tapes bonded with the polyurethane or the acrylic adhesive had faded substantially or completely degraded, with no loss of adhesion. The results of the 56 days of QUV exposure indicated that all of the rapid-set adhesives had very good to excellent adhesion on all surfaces (except for the epoxy 1 adhesive on Lexan polycarbonate); the acrylic adhesive attacked the Confirm ${ }^{\circledR}$ material, causing the security features to be faded and barely visible and the window area to be black or grayish-black at the beginning of the exposure; the security features of the tamper tapes bonded with the polyurethane adhesive were very faded after 4 to 7 weeks of exposure; the security features of the tamper tapes bonded with the epoxy 1 adhesive became badly faded after 2 to 8 weeks of exposure, depending on the surface; and the epoxy 2 adhesive-bonded tamper tapes had the best overall performance on all surfaces. The results of thermal cycling exposure for 56 days showed little or no effects to the security features of the tamper tapes (the acrylic-bonded tamper tapes did show slight to moderate fading of their security feature). The tapes displayed excellent adhesion, and their appearance with the acrylic and epoxy adhesive 1 changed only slightly. The tamper tapes held under control exposure conditions showed no change in appearance, adhesion, or security features during the 7 weeks of storage.

In the second study using rapid-set adhesives, tamper tapes with 2.54-cm-wide Confirm ${ }^{\circledR}$ windows were made in the laboratory to determine if another $3 \mathrm{M}$ Confirm ${ }^{\circledR}$ material might perform better than the 1700 series previously used in all other weathering studies. Three Confirm ${ }^{\circledR}$ materials, i.e., Confirm ${ }^{\circledR} 1500$ with primer, Confirm $1500^{\circledR}$ without primer, and Confirm $1300^{\circledR}$, were used. The tamper tapes were prepared as described for the first study using the same four candidate rapid-set adhesives. They were bonded to four surfaces, i.e., roughened aluminum, roughened steel, wood, and Mil. Spec. polyurethane-painted steel. They were tested at Florida, in the QUV. cabinet, and in the thermal cycling cabinet.

After exposure, the tamper tapes were examined as they were for the first study using reactive adhesives. The results from 2 months exposure at Florida indicated there was little to no change in adhesion, however, significant changes occurred in the security feature of the tamper tapes (the tamper tapes made of the Confirm ${ }^{\circledR} 1500$ materials turned black). The epoxy 2 adhesive produced the least amount of change in the visibility of the security features. The results from 42 days of exposure in the QUV cabinet indicated that, in general, the candidate adhesives performed better on tamper tapes prepared with the Confirm ${ }^{\circledR} 1300$ material than they did on the other 
Confirm ${ }^{\circledR}$ materials. The acrylic adhesive did not attack the 1300 material. The most loss in visibility of the security features was with the acrylic adhesive bonded to fiberglass board; otherwise, there was only slight or no change. Adhesion, in general, was very good. The epoxy 2 adhesive essentially displayed no change. The results of thermal cycling indicated that changes in temperature did not appear to affect the security feature or the adhesion of the tamper tapes nearly as much as did the conditions in the QUV cabinet. The tamper tapes, in general, held up very well in this exposure.

The overall results of the rapid-set adhesive weathering studies indicated that the epoxy 2 adhesive weathered better than the other adhesives on tamper tapes prepared with any of the Confirm ${ }^{\circledR}$ materials. 


\section{CONTENTS}

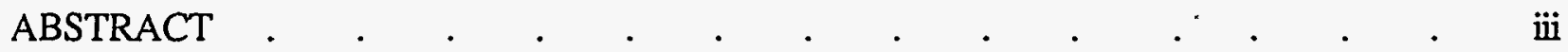

EXECUTIVE SUMMARY.

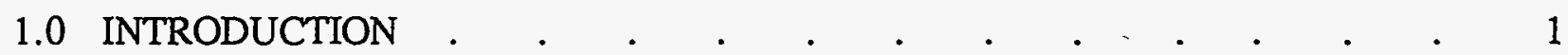

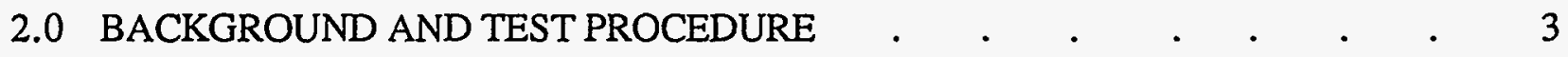

3.0 TAMPER TAPES WITH PRESSURE SENSITTVE ADHESIVE ～. • • . 7

3.1 1.27-CM-WIDE CONFIRM ${ }^{\circledR}$ WINDOW PROTOTYPE TAMPER TAPES 7

3.1 .1 Weathering . . . . . . $\quad$. . . . . 7

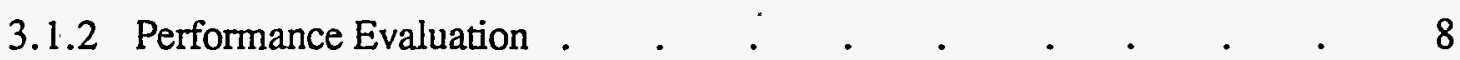

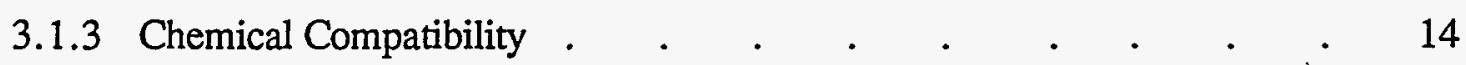

3.2 2.54-CM-WDE CONFIRM ${ }^{\circledR}$ WINDOW PROTOTYPE TAMPER TAPES 16

3.2 .1 Weathering . . . . . . . . . . . . . . 17

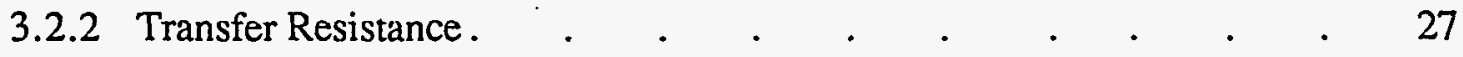

3.2.3 Abrasion Resistance . . . . . . . . . . . . . . 28

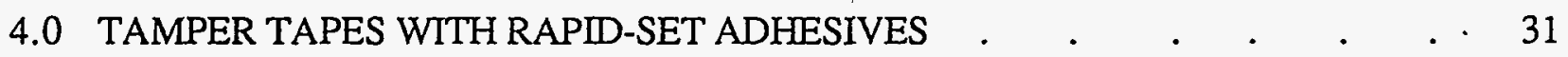

4.1 WEATHERING OF CONFIRM ${ }^{\circledR} 1700$ TAMPER TAPES . . . . . . 31

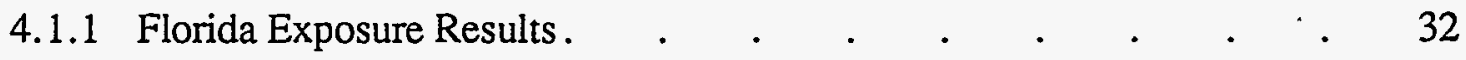

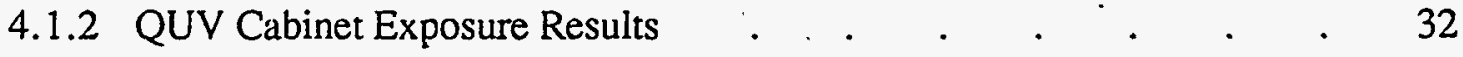

4.1.3 Thermal Cycling Exposure Results . . . . . . . . 40

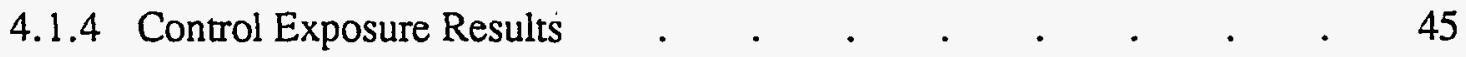

4.2 WEATHERING OF TAMPER TAPES MADE WITH THREE DIFFERENT CONFIRM ${ }^{\circledR}$ PRODUCTS .

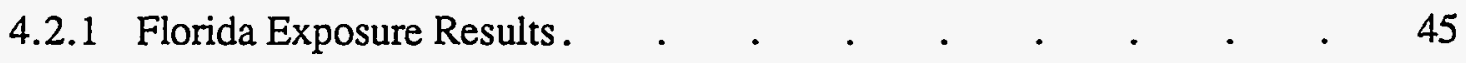

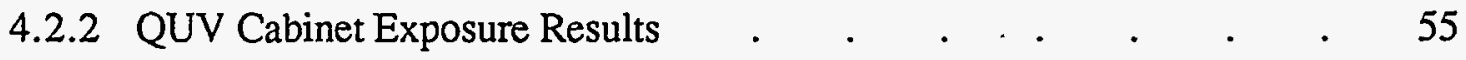

4.2.3 Thermal Cycling Exposure Results . . . . . . . 65

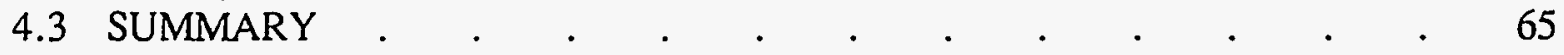




\section{LIST OF FIGURES}

2.1 Schematic Diagram of Basic Tamper Tape Design. . . . . . . . - 3

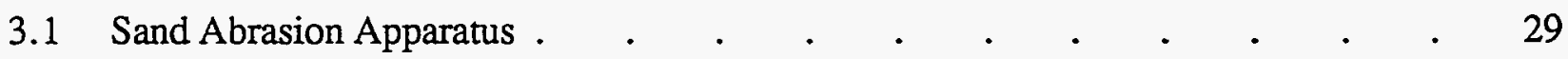

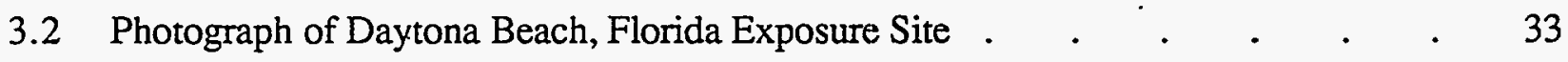

\section{LIST OF TABLES}

3.1 Results of Weathering in QUV Cabinet for 1.27-Centimeter-Wide Confirm ${ }^{\circledR}$

Window Tamper Tapes with Pressure Sensitive Adhesive Bonded to Various

Surfaces .

3.2 Results of Weathering in QUV Cabinet for 1.27-Centimeter-Wide Confirm ${ }^{\circledR}$

Window Tamper Tapes with Pressure Sensitive Adhesive Bonded to Various

Surfaces, $8 / 23 / 91$

3.3 Results of Weathering in Thermal Cycling Cabinet for 1.27-Centimeter-Wide Confirm ${ }^{\circledR}$ Window Tamper Tapes with Pressure Sensitive Adhesive Bonded to Various Surfaces, $8 / 23 / 91$

3.4 Results of Attempts to Remove the 1.27-Centimeter-Wide Confirm ${ }^{\circledR}$ Window Tamper Tapes with Pressure Sensitive Adhesive from Various Surfaces .

3.5 Results of Attempts to Remove the 1.27-Centimeter-Wide Confirm ${ }^{\circledR}$ Window Tamper Tapes with Pressure Sensitive Adhesive from Various Surfaces After QUV Weathering for at Least Seven Weeks . . . . . . . . . .

3.6 Results of Attempts to Remove the 1.27-Centimeter-Wide Confirm ${ }^{\circledR}$ Window Tamper Tapes with Pressure Sensitive Adhesive from Various Surfaces After Hot and Cold Thermal Cycling for at Least Seven Weeks . . . . . . 15

3.7 Effects of Chemical Exposure to Confirm ${ }^{\circledR}$ Tamper Tape Material . . . . $\quad 16$

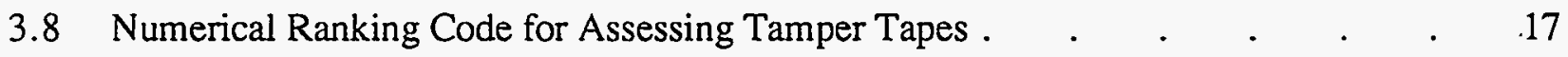

3.9 Results of Weather-O-Meter Exposure for 2.54-Centimeter-Wide Confirm ${ }^{\circledR}$

Window Tamper Tapes with Pressure Sensitive Adhesive and Polyester Underlay .

3.10 Results of Daytona Beach, Florida Exposure for 2.54-Centimeter-Wide Confirm ${ }^{\circledR}$ Window Tamper Tapes with Pressure Sensitive Adhesive and Polyester Underlay .

3.11 Results of QUV Cabinet Exposure for 2.54-Centimeter-Wide Confirm ${ }^{\circledR}$ Window Tamper Tapes with Pressure Sensitive Adhesive and Polyester Underlay.

3.12 Results of Thermal Cycling Exposure for 2.54-Centimeter-Wide Confirm ${ }^{\circledR}$ Window Tamper Tapes with Pressure Sensitive Adhesive and Polyester Underlay. . 


\section{TABLES (Continued)}

3.13 Results of Daytona Beach, Florida Exposure for 2.54-Centimeter-Wide Confirm ${ }^{\circledR}$ Window Tamper Tapes with Pressure Sensitive Adhesive and Vinyl Underlay .

3.14 Results of QUV Cabinet Exposure for 2.54-Centimeter-Wide Confirm ${ }^{\circledR}$ Window Tamper Tapes with Pressure Sensitive Adhesive and Vinyl Underlay

3.15 Results of Thermal Cycling Exposure for 2.54-Centimeter-Wide Confirm ${ }^{\circledR}$ Window Tamper Tapes with Pressure Sensitive Adhesive and Vinyl Underlay . . $\quad 26$

3.16 Summary of Transfer Resistance of 12/93 Prototype Tamper Tapes _ . . 28

3.17 Abrasion Resistance of 12/93 Prototype Tamper Tape with Sand . . . . 30

4.1 Evaluation of Tamper Tapes Applied with Candidate Rapid-Set Adhesives and Aged in Daytona Beach, Florida at $90^{\circ}$ South $\quad . \quad$. $\quad . \quad . \quad . \quad . \quad$.

4.2 Evaluation of Tamper Tapes Applied with Candidate Rapid-Set Adhesives and Aged in QUV Cabinet

4.3 Evaluation of Tamper Tapes Applied with Candidate Rapid-Set Adhesives and Aged in Thermal Cycling Cabinet

4.4 Evaluation of Tamper Tapes Applied with Candidate Rapid-Set Adhesives Held Under Control Conditions .

4.5 Evaluation of Tamper Tapes Prepared from Different Confirm ${ }^{\circledR}$ Materials, Applied with Candidate Rapid-Set Adhesives, and Exposed in Daytona Beach, Florida.

4.6 Evaluation of Confirm ${ }^{\circledR} 1500$ Without Primer Tamper Tapes Applied with Candidate Rapid-Set Adhesives and Aged in QUV Cabinet . . . . . 56

4.7 Evaluation of Confirm ${ }^{\circledR} 1500$ With Primer Tamper Tapes Applied with Candidate Rapid-Set Adhesives and Aged in QUV Cabinet . . . . . .

4.8 Evaluation of Confirm ${ }^{\circledR} 1300$ Tamper Tapes Applied with Candidate Rapid-Set Adhesives and Aged in QUV Cabinet .

4.9 Evaluation of Confirm ${ }^{\circledR} 1500$ Without Primer Tamper Tapes Applied with Candidate Rapid-Set Adhesives and Aged in the Thermal Cycling Cabinet

4.10 Evaluation of Confirm ${ }^{\circledR} 1500$ With Primer Tamper Tapes Applied with Candidate Rapid-Set Adhesives and Aged in the Thermal Cycling Cabinet

4.11 Evaluation of Confirm ${ }^{\circledR} 1300$ Tamper Tapes Applied with Candidate Rapid-Set Adhesives and Aged in the Thermal Cycling Cabinet 


\subsection{INTRODUCTION}

A tamper tape is an adhesive-backed label that possesses various tamper-indicating, transferresistant, or counterfeit-resistant properties. Tamper tapes are appealing for many applications because they are easy to use and are relatively robust. Readily and reliably applied to surfaces like an adhesive bandage, the tamper tape is practical and desirable for many scenarios. Applications include seals for temporary area denial, protection of sensitive equipment, chain-of-custody audit trails, and inventory control practices.

For the past few years, the Pacific Northwest Laboratory (PNL)a has investigated the development of tamper tapes for many applications. The objective of this ongoing work is to combine the best features of commercially-available tamper tapes with state-of-the-art design, unique identification, and counterfeit-resistant features. The PNL tamper tape is based on the patented Confirm ${ }^{\circledR}$ tamper-indicating technology developed and produced by $3 \mathrm{M}$ Company (Safety and Security Systems Division, 3M Center, St. Paul, Minnesota). Several interim prototype designs have been produced as more advanced features are developed. As part of this development, numerous prototype tamper tapes adhered to different surfaces have been evaluated under a various environmental aging conditions. The exposed tamper tapes were visually inspected and evaluated for transfer resistance to determine the effects of the weathering conditions. This report provides the results of these inspections and evaluations.

\footnotetext{
a Pacific Northwest Laboratory is operated by Battelle Memorial Institute for the U.S. Department of Energy under Contract DE-AC06-76RLO 1830.
} 



\subsection{BACKGROUND AND TEST PROCEDURES}

The basic layout and design of the tamper-indicating material of a Confirm ${ }^{\circledR}$ tamper tape is shown in Figure 2.1. The complete tamper tape consists of a top layer of Confirm ${ }^{\circledR}$ bonded to an underlay material (vinyl or polyester). The Confirm ${ }^{\circledR}$ top layer is made of glass beads embedded in a brittle bonding material. If transfer is attempted, the logo pattern reflected from beneath the

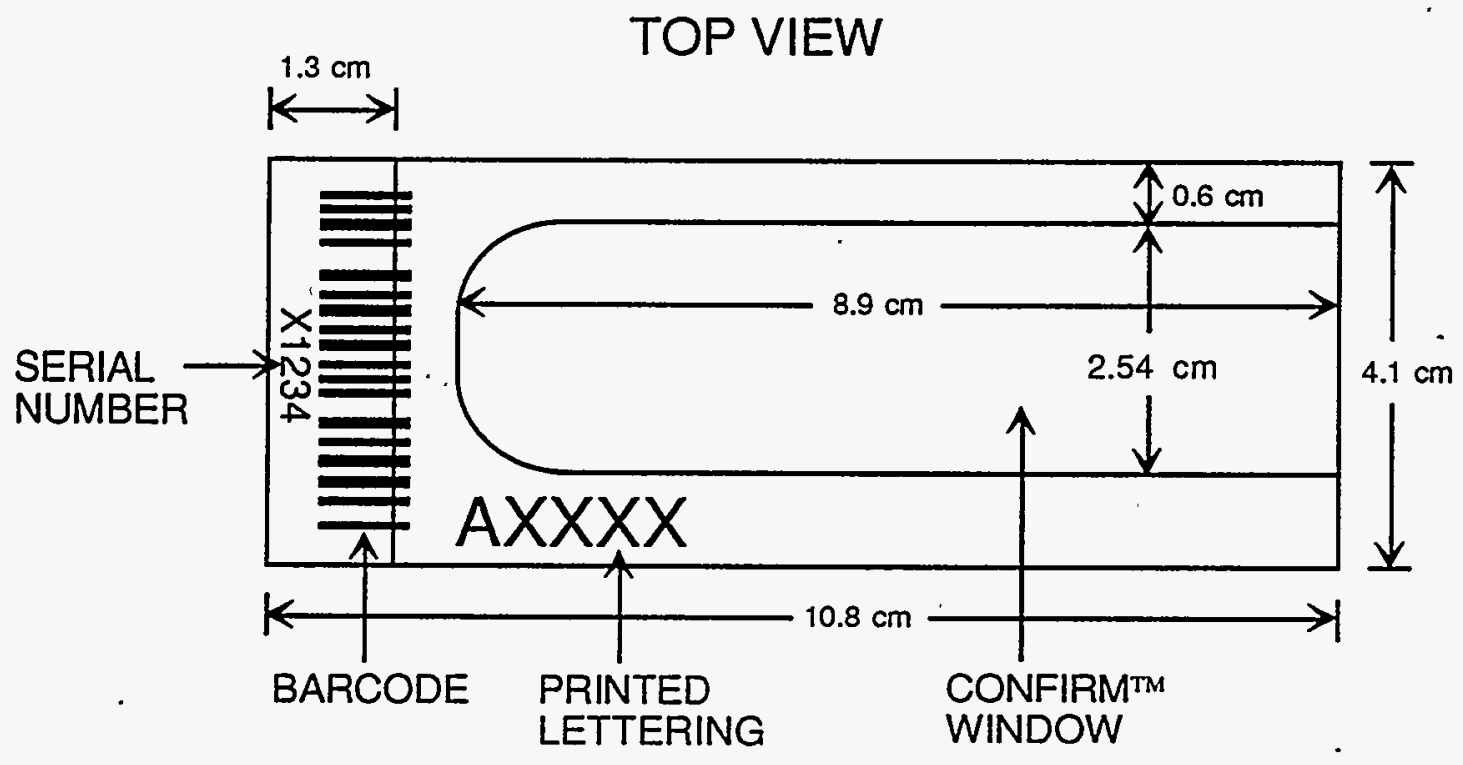

\section{ENLARGED SIDE VIEW}

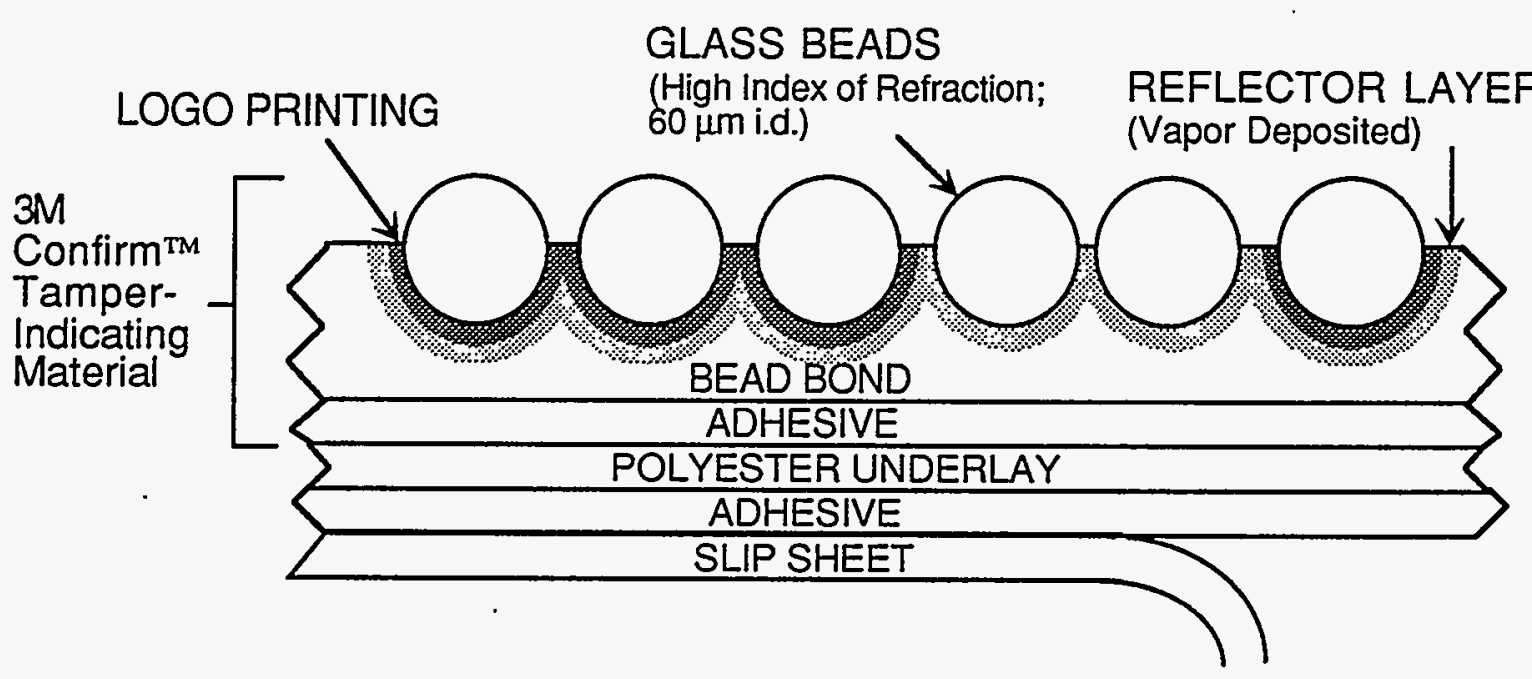

Figure 2.1 Schematic Diagram of Basic Tamper Tape Design 
glass beads is distorted as the beads are disrupted from the bonding layer. The integrity of the logo pattern is easily verified by visual observation when the tamper tape is illuminated with a light source (e.g., a flashlight) held perpendicular to the tape surface. Alterations to the tamperindicating material can be detected by examining the integrity of the logo printing.

The vinyl or polyester underlay material provides support around three sides of the tamper tape to allow the fragile Confirm ${ }^{\circledR}$ to be efficiently applied to a surface. The area where the polyester underlay is not present is known as the Confirm ${ }^{\circledR}$ window. The Confirm ${ }^{\circledR}$ window shown in Figure 2.1 is $2.54 \mathrm{~cm}$ wide with a narrow support frame. This is the current configuration that was chosen after several iterations of size and shape were evaluated for ease-ofuse and acceptable transfer resistance. Weathering studies were performed on prototype tamper tapes of this or a similar configuration $(5.1 \mathrm{~cm}$ by $10.2 \mathrm{~cm})$, as well as a previous configuration $(5.1 \mathrm{~cm}$ by $10.2 \mathrm{~cm})$ with a narrower Confirm ${ }^{\circledR}$ window $(1.27 \mathrm{~cm})$ and a wider support frame. Larger window regions render the tamper tape more transfer resistant, but also more difficult to apply.

In the configuration shown in Figure 2.1, the tamper tape is applied to a surface by removing the slip sheet and bonding with a pressure-sensitive adhesive (PSA). The PSA provides userfriendly application and generally bonds well to a wide variety of surface materials. Weathering studies were performed on tamper tapes of different configurations that used PSA for surface bonding. In initial studies, tamper tapes with PSA, 1.27-cm-wide Confirm ${ }^{\circledR}$ windows $\left(\right.$ Confirm ${ }^{\circledR}$ 1700 ), and a vinyl underlay were subjected to weathering in a QUV chamber, an outdoor exposure in a vertical south orientation in Daytona Beach, Florida, and cycling between hot and cold conditions (thermal cycling). The QUV chamber was set for cycling between 4 hours of simulated sunlight at $60^{\circ} \mathrm{C}$ and 4 hours of condensing humidity/moisture at $40^{\circ} \mathrm{C}$. The thermal cycling conditions were 12 hours at $-18^{\circ} \mathrm{C}$, followed by a 3-hour warming to $46^{\circ} \mathrm{C}, 6$ hours at $46^{\circ} \mathrm{C}$, and, finally, a 3-hour cooling to $-18^{\circ} \mathrm{C}$. Surfaces to which the tamper tapes were bonded included aluminum, steel, stainless steel, $\operatorname{Kevlar}^{\circledR}$, brass, copper, fiberglass/resin/gel coat, fiberglass/resin, and acrylonitrile:butadiene:styrene (ABS) plastic.

After weathering, the tamper tapes were visually inspected to determine the effects of the various environmental aging conditions. They were inspected at different time intervals for at least 7. weeks (up to 20 weeks) of exposure. The QUV-and thermal cycling-exposed tamper tapes were also subjected to a transfer resistance/performance evaluation to determine if they could be removed from the surfaces without disrupting the security feature. Methods used to determine transfer resistance included temperature extremes, sharp instruments, and chemicals/liquids. Tamper tapes of the same configuration (PSA, $1.27 \mathrm{~cm}$-wide Confirm ${ }^{\circledR}$ window, and vinyl underlay) bonded to 
a variety of surfaces but not exposed to any weathering conditions were also subjected to the same transfer resistance/performance evaluation.

The chemical compatibility of the Confirm ${ }^{\circledR}$ tamper tapes was investigated by applying the tapes to aluminum plates and then soaking them in various chemical liquids. Organic solvents used were ethanol, methylene chloride, chloroform, carbon tetrachloride, hexane, and dimethyl sulfoxide. Acids included concentrated nitric and sulfuric. Bases included two molar strengths of sodium hydroxide $(0.1 \mathrm{M}$ and $6 \mathrm{M})$ ): In addition, an oxidizing agent, hypochlorite (bleach), was used. The tamper tapes were visually evaluated immediately after exposure and again after 24 hours.

In later studies, after it was determined that a larger Confirm ${ }^{\circledR}$ window was necessary for adequate transfer resistance, weathering studies were performed on tamper tapes with PSA, 2.54-cm-wide Confirm ${ }^{\circledR}$ windows (Confirm ${ }^{\circledR} 1700$ ), and both polyester and vinyl underlay. The tamper tapes with polyester underlay (12/93) were bonded to four surfaces, i.e., aluminum, steel, polyester fiberglass board, and a Military Specification (Mil. Spec.) polyurethane-painted steel. They were exposed to weathering conditions in Daytona Beach, Florida, a Weather-O-Meter (continuous light but no water spray), the QUV chamber, and the thermal cycling cabinet. The tamper tapes with vinyl underlay were bonded to six surfaces, i.e., wood and Lexan polycarbonate in addition to those listed above, and were exposed to the same weathering conditions (with the exception of the Weather-O-Meter). These tamper tapes were also visually inspected to determine the effects of the various environmental aging conditions at different time intervals for at least 56 days to up to 5 months of exposure. The evaluation/examination of tamper tapes was done by two or three persons who provided a descriptive commentary on the tamper tapes' appearance (with and without the 3M security illuminator) and an evaluation of the adhesion of the tamper tapes' to the surfaces. The transfer resistance of the 12/93 prototype tamper tapes on four surfaces (aluminum, steel, fiberglass board, and polyurethane-painted steel) was evaluated after 14 days of exposure to QUV, thermal cycling, and control $\left(23^{\circ} \mathrm{C}, 50 \%\right.$ relative humidity) conditions. The abrasion resistance of the 12/93 tamper tapes using sand was also evaluated.

As further tamper tape development progressed, it was determined that increased security performance could be achieved by eliminating the PSA on the tamper tapes and substituting a more suitable reactive, rapid-set adhesive for surface bonding. Extensive adhesive formulation work was performed to obtain a rapid-set adhesive that had the best balance of (1) fast cure time, (2) good adhesion to a wide range of test surfaces, and (3) resistance to mechanical, heat, and solvent attack that may be used by an adversary to remove the tamper tape without damaging it. Formulation work was carried out using commercially available resin and hardening components 
that included three types of reactive adhesives: polyurethanes, epoxies, and acrylics. The rapid-set adhesives were evaluated by bonding tamper tapes to a wide variety of surfaces. Commerciallyavailable, two-component adhesives were also evaluated. As a result of these evaluations, four candidate rapid-set adhesives (two epoxies, one polyurethane, and one commercial acrylic) were chosen for weathering evaluation. Two weathering studies using tamper tapes bonded to surfaces using the four candidate rapid-set adhesives were performed.

In the first study using rapid-set adhesives, tamper tapes with 2.54-cm-wide Confirm ${ }^{\circledR}$ windows were made in the laboratory out of Confirm ${ }^{\circledR} 1700$ and vinyl underlay materials bonded together using a PSA supplied by $3 \mathrm{M}$. Each of the four candidate rapid-set adhesives were used to bond these tamper tapes to six surfaces, i.e., roughened steel, roughened aluminum, cedar wood, polyester fiberglass board, Lexan polycarbonate, and Mil. Spec. polyurethane-painted steel. The tamper tapes were cured for several days and then were tested in the QUV cabinet, the thermal cycling cabinet, in a constant temperature room $\left(23^{\circ} \mathrm{C}, 50 \%\right.$ relative humidity), and in Florida. The tamper tapes were then visually examined to assess their appearance, the appearance of the security feature with the $3 \mathrm{M}$ security illuminator, and the adhesion of the tamper tapes to the surfaces.

In the second study using rapid-set adhesives, tamper tapes with 2.54 -cm-wide Confirm ${ }^{\circledR}$ windows were made in the laboratory to determine if another $3 \mathrm{M}$ Confirm ${ }^{\circledR}$ material might perform better than the 1700 series previously used in all other weathering studies. Three Confirm ${ }^{\circledR}$ materials, i.e., Confirm ${ }^{\circledR} 1500$ with primer, Confirm $1500^{\circledR}$ without primer, and Confirm $1300^{\circledR}$, were used. The primary differences between these $3 \mathrm{M}$ products were in the bead-bond layers used. The 1700 products contained an "alkyd" layer, the 1500 products contained a polyurethane layer, and the 1300 product contained a "latex" layer. The tamper tapes were prepared as described for the first study using the same four candidate rapid-set adhesives. They were bonded to four surfaces, i.e., roughened aluminum, roughened steel, wood, and Mil. Spec. polyurethane-painted steel. They were tested in Florida, in the QUV cabinet, and in the thermal cycling cabinet. After exposure, the tamper tapes were examined just as they were in the first study using reactive adhesives.

Results of all of the weathering studies using tamper tapes with PSA (1.27- and 2.54-cmwide Confirm ${ }^{\circledR}$ windows, vinyl and polyester underlay) and tamper tapes with rapid-set adhesives (2.54-cm-wide Confirm ${ }^{\circledR}$ window, vinyl underlay) for surface bonding are given in the following sections. 


\subsection{TAMPER TAPES WITH PRESSURE SENSITIVE ADHESIVE}

During the course of the project, various prototype designs of Confirm ${ }^{\circledR}$ tamper tapes using PSA were evaluated. In initial studies, the Confirm ${ }^{\circledR}$ window was $1.27 \mathrm{~cm}$ wide, whereas in later studies, the Confirm ${ }^{\circledR}$ window was increased to $2.54 \mathrm{~cm}$ for added security reasons (i.e., the 1.27-cm-wide windows proved to have inadequate transfer-resistance characteristics). For most of the studies, a vinyl underlay material was used. However, for one prototype using PSA (12/93), a polyester underlay material was used. Various prototype tamper tapes bonded to many different surfaces were subjected to a variety of environmental aging conditions after which they were visually examined and tested for transfer resistance.

\subsection{27-CM-WIDE CONFIRM@ WINDOW PROTOTYPE TAMPER TAPES}

Two weathering studies of tamper tapes $(5.1 \mathrm{~cm}$ by $10.2 \mathrm{~cm})$ with $1.27-\mathrm{cm}$-wide Confirm ${ }^{\circledR}$ 1700 windows that used PSA for surface bonding were performed. After weathering, the tamper tapes were visually inspected to determine the effects of the environmental aging conditions. In addition, their tamper-resistance performance was evaluated.

\subsubsection{Weathering}

In the first sample set (7/23/91), two different tamper tapes, one with rounded corners and one with square corners (corresponding to different PSA), were each attached to the surfaces of panels made of aluminum, steel, and $\mathrm{Kevlar}^{\circledR}$. Several of the panels with attached tamper tapes were placed in a QUV chamber, which was set for cycling between 4 hours of simulated sunlight at $60^{\circ} \mathrm{C}$ and 4 hours of condensing humidity/moisture at $40^{\circ} \mathrm{C}$. Exposure lasted for 11 weeks. One of the aluminum panels was sent to the Battelle-Columbus, Daytona Beach, Florida laboratory for outdoor exposure in a vertical south orientation for 20 weeks.

In the second sample set (8/23/91), similar tamper tapes identified with (1) a larger letter "A" and (2) numbers only were attached to the surfaces of panels made from painted aluminum, painted steel, stainless steel, brass, copper, fiberglass/resin/gel coat (smooth front surface), fiberglass/resin (rough back surface), and ABS plastic. Part of these panels with attached tamper tapes were then exposed to the QUV conditions. The other part of the panels (exclusive of the painted aluminum and steel surfaces) were cycled between hot and cold conditions in a thermal cycling cabinet. The thermal cycling exposures involved 12 hours at $-18^{\circ} \mathrm{C}$, followed by a 3 -hour warming to $46^{\circ} \mathrm{C}, 6$ hours at $46^{\circ} \mathrm{C}$, and finally a 3 -hour cooling to $-18^{\circ} \mathrm{C}$. Exposures lasted for 7 weeks. 
Data showing the results of visual inspection of the first sample set exposures in the QUV chamber are given in Table 3.1. Data showing the results of visual inspection of the second sample set exposures in the QUV chamber are given in Table 3.2. Data showing the results of visual inspection of the second sample set exposures in the thermal cycling cabinet are given in Table 3.3. The data are summarized as follows:

- Slight yellowing of the tamper tapes was noted after the first day or two of exposure in the QUV cabinet. The amount of yellowing did not increase in the subsequent exposure time period.

- Under the QUV conditions, the steel panels rusted, as might be expected, and stained the tamper tapes.

- Adhesion to the rough fiberglass/resin surface without the gel coat (back) appeared to be poor under QUV conditions.

- On the tamper tapes exposed in the QUV cabinet, the security feature (3M's Confirm $\left.{ }^{\circledR}\right)$ appeared to "dim" over time and cracks appeared in the window areas near the conclusion of the tests.

- Hot/cold cycling had no effects that could be visually observed on the tamper tapes.

- The tamper tapes exposed in Florida showed slight yellowing, similar to the QUVexposed tamper tapes, and the square-cornered tamper tapes showed cracking in the upper corner after about 7 weeks.

An examination of the panel exposed to salt air at the Daytona, Florida laboratory after 20 weeks found that (1) the tamper tapes with the rounded corners remained as previously reported but mildew was growing on them, and (2) the tamper tapes with the square corners were beginning to "flake" in small areas and appeared to be losing adhesion to the surface.

\subsubsection{Performance Evaluation}

A variety of methods were investigated to determine if the prototype tamper tapes $(1.27-\mathrm{cm}-$ wide Confirm ${ }^{\circledR}$ window, bonded with PSA) could be removed from various surfaces without disrupting the security feature. These methods included the use of temperature extremes, sharp instruments, and chemicals/liquids. The transfer resistance of the tamper tapes with and without exposure to weathering conditions was evaluated.

\section{Transfer Evaluations of Tamper Tapes Without Weathering}

In the evaluation of the tamper resistance of the tamper tapes without weathering, no attempts were made to evaluate the influence of set time. Tamper resistance. was attempted by numerous 
Table 3.1. Results of Weathering in QUV Cabinet for 1.27-Centimeter-Wide Confirm ${ }^{\circledR}$ Window Tamper Tapes with Pressure Sensitive Adhesive Bonded to Various Surfaces, 7/23/91

\begin{tabular}{|c|c|c|c|c|c|c|c|c|c|c|c|c|c|c|}
\hline \multirow[b]{2}{*}{$\begin{array}{l}\text { Surface } \\
\text { (Ident. No.b) }\end{array}$} & \multicolumn{14}{|c|}{ Exposure Time } \\
\hline & $\begin{array}{c}1 \\
\text { Day } \\
\end{array}$ & $\begin{array}{c}6 \\
\text { Days } \\
\end{array}$ & $\begin{array}{c}8 \\
\text { Days } \\
\end{array}$ & $\begin{array}{c}10 \\
\text { Days }\end{array}$ & $\begin{array}{c}13 \\
\text { Days }\end{array}$ & $\begin{array}{c}3 \\
\text { Wks } \\
\end{array}$ & $\begin{array}{c}4 \\
4 \\
\text { Wks } \\
\end{array}$ & $\begin{array}{c}5 \\
\text { Wks } \\
\end{array}$ & $\begin{array}{c}6 \\
\text { Wks } \\
\end{array}$ & $\begin{array}{c}7 \\
\text { Wks } \\
\end{array}$ & $\begin{array}{c}8 \\
\text { Wks } \\
\end{array}$ & $\begin{array}{c}9 \\
\text { Wks } \\
\end{array}$ & $\begin{array}{c}10 \\
\text { Wks } \\
\end{array}$ & $\begin{array}{c}11 \\
\text { Wks } \\
\end{array}$ \\
\hline Aluminum (A41) & SY & $\mathrm{NC}$ & $\mathrm{NC}$ & $\mathrm{NC}$ & NC & NC & $\mathrm{NC}$ & $\mathrm{NC}$ & $\mathrm{NC}$ & $\mathrm{NC}$ & $\mathrm{NC}$ & $\mathrm{NC}$ & $\mathrm{NC}$ & NC \\
\hline Aluminum (A42) & $\mathrm{NC}$ & SY & $\mathrm{NC}$ & $\mathrm{NC}$ & $\mathrm{NC}$ & NC & $\mathrm{NC}$ & NC & $\mathrm{NC}$ & $\mathrm{NC}$ & $\mathrm{NC}$ & $\mathrm{NC}$ & $\mathrm{NC}$ & $\mathrm{NC}$ \\
\hline Kevlar ${ }^{\circledR}(\mathrm{K} 41)$ & SY & $\mathrm{NC}$ & $\mathrm{NC}$ & $\mathrm{NC}$ & $\mathrm{NC}$ & $\mathrm{NC}$ & $\mathrm{NC}$ & $\mathrm{NC}$ & $\mathrm{NC}$ & $\mathrm{NC}$ & NC & $\mathrm{NC}$ & $\mathrm{NC}$ & $\mathrm{NC}$ \\
\hline Kevlar $^{(\mathbb{B}}(\mathrm{K} 42)$ & $\mathrm{NC}$ & SY & $\mathrm{NC}$ & $\mathrm{NC}$ & $\mathrm{NC}$ & $\mathrm{NC}$ & $\mathrm{NC}$ & $\mathrm{NC}$ & $\mathrm{NC}$ & $\mathrm{NC}$ & $\mathrm{NC}$ & $\mathrm{NC}$ & $\mathrm{NC}$ & $\mathrm{NC}$ \\
\hline Steel (S51) & $S Y, R$ & NC, R & $\mathrm{NC}$ & $\mathrm{NC}$ & $\mathrm{NC}$ & $\mathrm{NC}$ & $\mathrm{NC}$ & $\mathrm{NC}$ & $\mathrm{NC}$ & $\mathrm{NC}$ & $\mathrm{NC}$ & $\mathrm{NC}$ & $\mathrm{NC}$ & $\mathrm{NC}$ \\
\hline Steel (S52) & $\mathrm{NC}, \mathrm{R}$ & SY,R & $\mathrm{NC}$ & $\mathrm{NC}$ & $\mathrm{NC}$ & $\mathrm{NC}$ & $\mathrm{NC}$ & $\mathrm{NC}$ & $\mathrm{NC}$ & $\mathrm{NC}$ & $\mathrm{NC}$ & $\mathrm{NC}$ & $\mathrm{NC}$ & $\mathrm{NC}$ \\
\hline
\end{tabular}

a $\mathrm{S}=$ slight; $\mathrm{Y}=$ yellowing; $\mathrm{NC}=$ no change from previous evaluation; $\mathrm{R}=$ rusting

b 41 and 51 indicate tamper tapes with rounded corners; 42 and 52 indicate tamper tapes with square corners 
Table 3.2. Results of Weathering in QUV Cabinet for 1.27-Centimeter-Wide Confirm ${ }^{\circledR}$ Window Tamper Tapes with Pressure Sensitive Adhesive Bonded to Various Surfaces, 8/23/91

\begin{tabular}{|c|c|c|c|c|c|c|c|}
\hline \multirow{2}{*}{$\begin{array}{l}\text { Surface } \\
\text { (Ident. No.b) }\end{array}$} & \multicolumn{7}{|c|}{ Exposure Timea } \\
\hline & 12 Days & 17 Days & 23 Days & 30 Days & 5 Weeks & 6 Weeks & 7 Weeks \\
\hline $\begin{array}{l}\text { Painted Aluminum } \\
\text { (A31) }\end{array}$ & SY & $\mathrm{NC}$ & $\mathrm{NC}$ & $\mathrm{NC}$ & $\mathrm{NC}$ & $\mathrm{NC}$ & $\mathrm{NC}$ \\
\hline $\begin{array}{l}\text { Painted Aluminum } \\
\text { (A32) }\end{array}$ & SY & NC & NC & NC & NC & $\mathrm{NC}$ & $\mathrm{NC}$ \\
\hline Painted Steel (S11) & SY & $\mathrm{NC}$ & $\mathrm{NC}$ & $\mathrm{NC}$ & $\mathrm{NC}$ & $\mathrm{NC}$ & $\mathrm{NC}$ \\
\hline Painted Steel (S12) & SY & $\mathrm{NC}$ & $\mathrm{NC}$ & $\mathrm{NC}$ & NC & $\mathrm{NC}$ & $\mathrm{NC}$ \\
\hline $\begin{array}{l}\text { Stainless Steel } \\
\text { (SS1AQ) }\end{array}$ & SY & NC & $\mathrm{NC}$ & NC & $\mathrm{NC}$ & $\mathrm{NC}$ & $\mathrm{NC}$ \\
\hline $\begin{array}{l}\text { Stainless Steel } \\
\text { (SS 1BQ) }\end{array}$ & SY & $\mathrm{NC}$ & $\mathrm{NC}$ & NC & $\mathrm{NC}$ & NC & $\mathrm{NC}$ \\
\hline Brass (B1AQ) & SY & $\mathrm{NC}$ & $\mathrm{NC}$ & NC & $\mathrm{NC}$ & $\mathrm{NC}$ & $\mathrm{NC}$ \\
\hline Brass (B $1 \mathrm{BQ})$ & SY & $\mathrm{NC}$ & $\mathrm{NC}$ & $\mathrm{NC}$ & $\mathrm{NC}$ & $\mathrm{NC}$ & $\mathrm{NC}$ \\
\hline Copper (C1AQ) & SY & $\mathrm{NC}$ & $\mathrm{NC}$ & $\mathrm{NC}$ & $\mathrm{NC}$ & $\mathrm{NC}$ & $\mathrm{NC}$ \\
\hline Copper (C1BQ) & SY & NC & NC & NC & $\mathrm{NC}$ & $\mathrm{NC}$ & $\mathrm{NC}$ \\
\hline $\begin{array}{l}\text { Fiberglass/resin, } \\
\text { back (P1AQB) }\end{array}$ & $S Y$ & $\mathrm{NC}$ & PA & $\mathrm{NC}$ & $\mathrm{NC}$ & $\mathrm{NC}$ & $\mathrm{NC}$ \\
\hline $\begin{array}{l}\text { Fiberglass/resin, } \\
\text { back (P1BQB) }\end{array}$ & SY & $\mathrm{NC}$ & PA & NC & SPL & $\mathrm{NC}$ & $\mathrm{NC}$ \\
\hline $\begin{array}{l}\text { Fiberglass/resin, } \\
\text { front }(\mathrm{P} 2 \mathrm{AQF})\end{array}$ & $S Y$ & $\mathrm{NC}$ & $\mathrm{NC}$ & NC & $\mathrm{NC}$ & $\mathrm{NC}$ & $\mathrm{NC}$ \\
\hline $\begin{array}{l}\text { Fiberglass/resin, } \\
\text { front (P2BQF) }\end{array}$ & SY & $\mathrm{NC}$ & $\mathrm{NC}$ & NC & $\mathrm{NC}$ & $\mathrm{NC}$ & $\mathrm{NC}$ \\
\hline $\begin{array}{c}\text { ABS Plastic } \\
\text { (ABSIAQ) }\end{array}$ & SY & $\mathrm{NC}$ & $\mathrm{NC}$ & $\mathrm{NC}$ & $\mathrm{NC}$ & NC & $\mathrm{NC}$ \\
\hline $\begin{array}{l}\text { ABS Plastic } \\
\text { (ABS1BQ) }\end{array}$ & SY & $\mathrm{NC}$ & $\mathrm{NC}$ & $\mathrm{NC}$ & $\mathrm{NC}$ & $\mathrm{NC}$ & $\mathrm{NC}$ \\
\hline $\begin{array}{l}\text { a } \mathrm{PA}=\text { poor adhe } \\
\mathrm{NC}=\text { no chang }\end{array}$ & $\begin{array}{l}\overline{=w i} \\
\text { vious }\end{array}$ & $\begin{array}{l}\text { was sp } \\
\text { dation }\end{array}$ & $\overline{\text { igth }}$ & abov & ode; & $=$ slig & lowing; \\
\hline $\begin{array}{l}\text { b Identified as foll } \\
\mathrm{P}=\text { fiberglass } / \mathrm{re} \\
\text { "A"; } 1 \mathrm{~B}=\operatorname{tamp} \\
\mathrm{F}=\text { front of pan } \\
\text { comers; and } 11\end{array}$ & alum & ; S $=\mathrm{st}$ & $S=$ & less & $\begin{array}{l}3=b \\
=t a\end{array}$ & $\begin{array}{l}\mathrm{C}=\mathrm{co} \\
\mathrm{tapes} \\
\mathrm{B}=\mathrm{b}\end{array}$ & $\begin{array}{l}\text { th } \\
\text { th a large } \\
\text { k of panel; } \\
\text { ith square }\end{array}$ \\
\hline
\end{tabular}


Table 3.3. Results of Weathering in Thermal Cycling Cabinet for 1.27-Centimeter-Wide Confirm ${ }^{\circledR}$ Window Tamper Tapes with Pressure Sensitive Adhesive Bonded to Various Surfaces, 8/23/91

\begin{tabular}{|c|c|c|c|c|c|c|c|}
\hline \multirow{2}{*}{$\begin{array}{l}\text { Surface } \\
\text { (Ident. No.b) }\end{array}$} & \multicolumn{7}{|c|}{ Exposure Time } \\
\hline & 12 Days & 17 Days & 23 Days & 30 Days & 5 Weeks & 6 Weeks & 7 Weeks \\
\hline $\begin{array}{l}\text { Stainless Steel } \\
\text { (SS2AC) }\end{array}$ & $\mathrm{NC}$ & $\mathrm{NC}$ & $\mathrm{NC}$ & $\mathrm{NC}$ & NC & $\mathrm{NC}$ & $\mathrm{NC}$ \\
\hline $\begin{array}{l}\text { Stainless Steel } \\
\text { (SS2BC) }\end{array}$ & $\mathrm{NC}$ & $\mathrm{NC}$ & NC & $\mathrm{NC}$ & NC & NC & $\mathrm{NC}$ \\
\hline Brass (B2AC) & NC & $\mathrm{NC}$ & $\mathrm{NC}$ & NC & NC & $\mathrm{NC}$ & $\mathrm{NC}$ \\
\hline Brass (B2BC) & NC & $\mathrm{NC}$ & $\mathrm{NC}$ & $\mathrm{NC}$ & NC & $\mathrm{NC}$ & $\mathrm{NC}$ \\
\hline Copper (C2AC) & NC & $\mathrm{NC}$ & NC & NC & $\mathrm{NC}$ & $\mathrm{NC}$ & $\mathrm{NC}$ \\
\hline Copper (C2BC) & $\mathrm{NC}$ & $\mathrm{NC}$ & $\mathrm{NC}$ & NC & $\mathrm{NC}$ & $\mathrm{NC}$ & NC \\
\hline $\begin{array}{c}\text { Fiberglass/resin, } \\
\text { back (P1ACB) }\end{array}$ & NC & $\mathrm{NC}$ & $\mathrm{NC}$ & $\mathrm{NC}$ & $\mathrm{NC}$ & NC & $\mathrm{NC}$ \\
\hline $\begin{array}{c}\text { Fiberglass/resin, } \\
\text { back (PIBCB) }\end{array}$ & $\mathrm{NC}$ & NC & $\mathrm{NC}$ & NC & $\mathrm{NC}$ & $\mathrm{NC}$ & $\mathrm{NC}$ \\
\hline $\begin{array}{l}\text { Fiberglass/resin, } \\
\text { front (P2ACF) }\end{array}$ & $\mathrm{NC}$ & $\mathrm{NC}$ & $\mathrm{NC}$ & NC & $\mathrm{NC}$ & $\mathrm{NC}$ & $\mathrm{NC}$ \\
\hline $\begin{array}{l}\text { Fiberglass/resin, } \\
\text { front }(\mathrm{P} 2 \mathrm{BCF})\end{array}$ & NC & $\mathrm{NC}$ & $\mathrm{NC}$ & $\mathrm{NC}$ & $\mathrm{NC}$ & NC & $\mathrm{NC}$ \\
\hline $\begin{array}{l}\text { ABS Plastic } \\
\text { (ABS2AC) }\end{array}$ & $\mathrm{NC}$ & NC & $\mathrm{NC}$ & $\mathrm{NC}$ & NC & $\mathrm{NC}$ & NC \\
\hline $\begin{array}{l}\text { ABS Plastic } \\
(\mathrm{ABS} 2 \mathrm{BC}) \\
\end{array}$ & $\mathrm{NC}$ & $\mathrm{NC}$ & $\mathrm{NC}$ & $\mathrm{NC}$ & $\mathrm{NC}$ & $\mathrm{NC}$ & $\mathrm{NC}$ \\
\hline
\end{tabular}

methods of removal from various steel and aluminum surfaces as listed in Table 3.4. The results of the evaluations are also given in Table 3.4.

In general, the most straightforward method for removing the tamper tapes was to use a razor blade and carefully peel the tamper tape off the surface. On the aluminum, the tamper tape could be removed with the adhesive layer intact and without destroying the security feature. Therefore, it was possible to re-adhere the removed tamper tape to another surface. It was slightly more 
Table 3.4. Results of Attempts to Remove the 1.27-Centimeter-Wide Tamper Tapes with Pressure Sensitive Adhesive from Various Surfaces

\begin{tabular}{|c|c|c|c|c|c|c|c|c|}
\hline $\begin{array}{l}\text { Method of } \\
\text { Removal }\end{array}$ & $\begin{array}{l}\text { Type of } \\
\text { Surface } \\
\text { (Ident.) }\end{array}$ & $\begin{array}{l}\text { Security } \\
\text { Feature } \\
\text { Destroyed }\end{array}$ & $\begin{array}{c}\text { Causeda } \\
\text { Tamper Tape } \\
\text { Release }\end{array}$ & $\begin{array}{c}\text { Caused }^{b} \\
\text { Adhesive } \\
\text { Release }\end{array}$ & $\begin{array}{l}\text { Destroyedc } \\
\text { Printing }\end{array}$ & $\begin{array}{l}\text { Destroyed } \\
\text { Tamper } \\
\text { Tapes } \\
\end{array}$ & $\begin{array}{l}\text { Tamper } \\
\text { Tapes } \\
\text { Applied } \\
\text { In Lab } \\
\end{array}$ & $\begin{array}{l}\text { Overall } \\
\text { Rating }\end{array}$ \\
\hline Hotplate & steel (S6) & yes & yes & no & & yes & & 3 \\
\hline Freezer & aluminum (A7) & no & no & slight & & no & & 4 \\
\hline Quick freeze & aluminum (A6) & no & no & no & & yes & & 5 \\
\hline Hot air gun & steel (S6) & no & no & no & no & no & $*$ & 5 \\
\hline Heat/Quick freeze & steel (S6) & no & no & no & & no & . & 5 \\
\hline Razor blade & $\begin{array}{l}\text { aluminum (A6) } \\
\text { steel (S6) }\end{array}$ & $\begin{array}{l}\text { no } \\
\text { no }\end{array}$ & $\begin{array}{l}\text { no } \\
\text { no }\end{array}$ & $\begin{array}{l}\text { yes } \\
\text { yes }\end{array}$ & no & $\begin{array}{l}\text { no } \\
\text { yes }\end{array}$ & * & $\begin{array}{l}1 \\
2\end{array}$ \\
\hline Hot soapy water & aluminum (A7) & no & no & slight & yes, black & no & * & 4 \\
\hline Hot coffee/creamer & aluminum (A6) & no & no & slight & yes, black & no & * & 4 \\
\hline Tilex & steel (S6) & no & no & slight & & no & & 4 \\
\hline Acetone & $\begin{array}{l}\text { aluminum (A6) } \\
\text { steel (S6) }\end{array}$ & $\begin{array}{l}\text { yes } \\
\text { yes }\end{array}$ & $\begin{array}{l}\text { no } \\
\text { no }\end{array}$ & $\begin{array}{l}\text { yes } \\
\text { yes }\end{array}$ & & $\begin{array}{l}\text { yes } \\
\text { yes }\end{array}$ & * & $\begin{array}{l}5 \\
5\end{array}$ \\
\hline Methyl Ethyl Ketone & $\begin{array}{l}\text { aluminum (A6) } \\
\text { steel (S6) }\end{array}$ & $\begin{array}{l}\text { yes } \\
\text { yes }\end{array}$ & $\begin{array}{l}\text { no } \\
\text { no }\end{array}$ & $\begin{array}{l}\text { yes } \\
\text { yes }\end{array}$ & & $\begin{array}{l}\text { yes } \\
\text { yes }\end{array}$ & $*$ & $\begin{array}{l}5 \\
5\end{array}$ \\
\hline Xylene & $\begin{array}{l}\text { aluminum (A6) } \\
\text { steel (S6) }\end{array}$ & $\begin{array}{l}\text { yes } \\
\text { yes }\end{array}$ & $\begin{array}{l}\text { no } \\
\text { no }\end{array}$ & $\begin{array}{l}\text { yes } \\
\text { yes }\end{array}$ & & $\begin{array}{l}\text { yes } \\
\text { yes }\end{array}$ & * & $\begin{array}{l}5 \\
5\end{array}$ \\
\hline
\end{tabular}

a Tamper tapes separated from adhesive which remained on surface

b Adhesive separated from surface (entire tamper tapes' structure removed)

c Some tamper tapes did not have printing

d A quick reference for method of removal: 1, most advantageous (i.e., easiest to remove), through 5, least advantageous (i.e., most difficult to remove) 
Table 3.4. Continued

\begin{tabular}{|c|c|c|c|c|c|c|c|c|}
\hline $\begin{array}{l}\text { Method of } \\
\text { Removal }\end{array}$ & $\begin{array}{l}\text { Type of } \\
\text { Surface } \\
\text { (Ident.) }\end{array}$ & $\begin{array}{c}\text { Security } \\
\text { Feature } \\
\text { Destroyed }\end{array}$ & $\begin{array}{c}\text { Causeda } \\
\text { Tamper Tape } \\
\text { Release } \\
\end{array}$ & $\begin{array}{l}\text { Caused } \\
\text { Adhesive } \\
\text { Release }\end{array}$ & $\begin{array}{c}\text { Destroyedc } \\
\text { Printing }\end{array}$ & $\begin{array}{l}\text { Destroyed } \\
\text { Tamper } \\
\text { Tapes }\end{array}$ & $\begin{array}{c}\text { Tamper } \\
\text { Tapes } \\
\text { Applied } \\
\text { In Lab } \\
\end{array}$ & $\begin{array}{l}\text { Overalld } \\
\text { Rating }\end{array}$ \\
\hline Methylene chloride & aluminum (A7) & yes & no & yes & yes & yes & $*$ & 15 \\
\hline Tetrahydrofuran & aluminum (A7) & yes & yes & yes & yes & yes & * & 5 \\
\hline Turpsol & aluminum (A7) & yes & no & slight & yes & yes & * & 5 \\
\hline Aromatic 100 & aluminum (A7) & yes & yes & no & yes & yes & * & 5 \\
\hline Dimethyl formamide & steel (S6) & yes & yes & no & yes & yes & * & 5 \\
\hline Mineral oil & aluminum (S6) & no & no & slight & no & no & & 4 \\
\hline Silicone oil & aluminum (S6) & no & no & no & no & no & $*$ & 5 \\
\hline Mold release & aluminum (S6) & no & no & no & no & no & $*$ & 5 \\
\hline $1 \% \mathrm{NaOH}$ & aluminum (A7) & yes & no & slight & yes & yes & $*$ & 5 \\
\hline $1 \%$ Sulfuric acid & aluminum (A7) & yes & no & no & yes & yes & * & 5 \\
\hline Methanol & aluminum (A6) & yes & no & no & & yes & $*$ & 5 \\
\hline Isopropyl alcohol & aluminum (A6) & yes & no & no & . & yes & * & 5 \\
\hline Razor blarin & $\begin{array}{l}\text { sand blasted } \\
\text { aluminum }\end{array}$ & no & no & yes & no & no & * & 1 \\
\hline
\end{tabular}

a Tamper tapes separated from adhesive which remained on surface

b Adhesive separated from surface (entire tamper tapes structure removed)

c Some tamper tapes did not have printing

d A quick reference for method of removal: 1, most advantageous (i.e., easiest to remove), through 5, least advantageous (i.e., most difficult to remove) 
difficult to remove the tamper tape from the steel. In this attempt, the window area was slightly damaged. However, the results indicated that with care and patience these prototype tamper tapes most likely could be removed and are, therefore, not secure against this threat.

Various readily-accessible chemicals and liquids were evaluated to determine if they improved the ease with which the tamper tape could be removed. A variety of solvents, oils, and acidic and caustic solutions were applied to the tamper tape adhesive interface while peeling the tamper tape from the substrate. None of the evaluated materials significantly enhanced the removal process. In addition, many of the materials caused the inks on the printed tamper tapes to bleed.

The use of heat and extreme cold were also investigated to determine if they aided in the removal of the tamper tapes. These conditions did not make it easier to remove the tamper tapes to any significant extent.

\section{Transfer Evaluation of Weathered Tamper Tapes}

Tamper tapes exposed to the weathering conditions of the QUV and thermal cycling cabinets were also subjected to transfer resistance/performance evaluation analyses after the exposure time period (a minimum of 7 weeks, up to 11 weeks). The tamper tapes that were exposed in the QUV cabinet were evaluated while they were still wet from the condensation cycle and again after they had dried. The QUV results are listed in Table 3.5. All of the tamper tapes (except on the copper) were damaged during the removal attempts using a razor blade. It appeared that the damage was due to the weathering effects. The tamper tapes became very fragile and brittle during the exposure in the QUV cabinet. The weathering was severe enough, in some cases, to cause cracking around the transparent window. These cracks could be incorrectly perceived as a tampering attempt when interrogated during use in the field.

The results of the transfer-resistance evaluations for the tamper tapes exposed to the hot and cold cycles in the thermal cycling cabinet are given in Table 3.6. Most of the tamper tapes were damaged during the removal attempts with a razor blade. However, these tamper tapes did not appear to become fragile like the tamper tapes exposed in the QUV cabinet. It may have been more difficult to remove these tamper tapes just because they were on the surface for a longer period of time.

\subsubsection{Chemical Compatibility}

The chemical compatibility of the Confirm ${ }^{\circledR}$ tamper tapes was investigated by applying the tapes to aluminum plates and then soaking the plates in various chemicals. Chemicals included organic solvents, acids, bases, and oxidizing liquids. The tamper tapes were visually examined 
Table 3.5. Results of Attempts to Remove the 1.27-Centimeter-Wide Confirm ${ }^{\circledR}$ Window Tamper Tapes with Pressure Sensitive Adhesive from Various Surfaces After QUV Weathering for at Least Seven Weeks

\begin{tabular}{lllll}
\hline Ident. No. & \multicolumn{1}{c}{$\begin{array}{c}\text { Surface (Weeks of } \\
\text { Exposure) }\end{array}$} & $\begin{array}{c}\text { Method of } \\
\text { Removal }\end{array}$ & $\begin{array}{c}\text { Removed Tamper } \\
\text { Tape }\end{array}$ & Damageda \\
\hline $45881-9-1$ & Brass (7) & razor & yes & yes \\
$45881-9-2$ & Stainless Steel (7) & razor & yes & yes \\
$45881-9-3$ & ABS Plastic (7) & razor & yes & yes \\
$45881-10-1$ & Fiberglass back (7) & razor & yes & yes \\
$45881-10-2$ & Painted Aluminum (7) & razor & yes & yes \\
$45881-10-3$ & Kevlar ${ }^{\circledR}(11)$ & razor & yes & yes \\
$45881-10-4$ & Copper (7) & razor & yes & no \\
$45881-11-1$ & Steel (11) & razor & yes & yes \\
$45881-11-2$ & Painted Steel (7) & razor & yes & yes \\
$45881-11-3$ & Fiberglass front (7) & razor & yes & yes \\
$45881-11-4$ & Aluminum (11) & razor & yes & yes \\
\hline
\end{tabular}

a The accelerated weathering affected all the samples in that it diminished the brightness of the security feature, caused cracking in the transparent strip, and made the strip brittle

Table 3.6. Results of Attempts to Remove the 1.27-Centimeter-Wide Confirm ${ }^{\circledR}$ Window Tamper Tapes with Pressure Sensitive Adhesive from Various Surfaces After Hot and Cold Thermal Cycling for at Least Seven Weeks

\begin{tabular}{|c|c|c|c|}
\hline Surface & Method of Removal & $\begin{array}{c}\text { Removed } \\
\text { Tamper Tape }\end{array}$ & Damaged \\
\hline Brass & razor & yes & yes \\
\hline Copper & razor. & yes & yes \\
\hline ABS Plastic & razor & yes & no \\
\hline Stainless Steel & razor & yes & yes \\
\hline Fiberglass/resin back (rough) & razor & yes & yes \\
\hline Fiberglass/resin (smooth) & razor & yes & no \\
\hline
\end{tabular}


immediately after exposure and after 24 hours of exposure to determine the effects of the various chemicals. The results are summarized in Table 3.7.

\subsection{54-CM-WIDE CONFIRM@ WINDOW PROTOTYPE TAMPER TAPES}

Since transfer-resistance/performance evaluations of the 1.27 -cm-wide Confirm $^{\circledR}$ window prototype tamper tapes indicated it was likely they could be removed without evidence of tampering, prototype tamper tapes with a larger $\operatorname{Confirm}^{\circledR}$ window were developed and evaluated. Two prototype tamper tapes with $2.54-\mathrm{cm}$-wide Confirm ${ }^{\circledR} 1700$ windows were subjected to environmental aging exposures. One had a polyester underlay and the other a vinyl underlay.

Table 3.7. Effects of Chemical Exposure to Confirm ${ }^{\circledR}$ Tamper Tape Material

\begin{tabular}{|c|c|c|}
\hline Exposure Chemical & Immediate Effect & After 24 Hour Exposure \\
\hline Ethanol & $\begin{array}{l}\text { No discoloration or deformation, } \\
\text { no softening of adhesive }\end{array}$ & No change \\
\hline Mẹthylene Chloride & $\begin{array}{l}\text { No discoloration or deformation, } \\
\text { significant softening of adhesive }\end{array}$ & No change \\
\hline Chloroform & $\begin{array}{l}\text { No discoloration or deformation, } \\
\text { significant softening of adhesive }\end{array}$ & No.change \\
\hline Carbon Tetrachloride & $\begin{array}{l}\text { No discoloration or deformation, } \\
\text { no softening of adhesive }\end{array}$ & No. change \\
\hline Hexane & $\begin{array}{l}\text { No discoloration or deformation, } \\
\text { no softening of adhesive }\end{array}$ & $\begin{array}{l}\text { Discoloration, no adhesive } \\
\text { softening }\end{array}$ \\
\hline Dimethylsulfoxide & $\begin{array}{l}\text { Printing discolored, adhesive } \\
\text { softened }\end{array}$ & No additional damage \\
\hline $0.1 \mathrm{M}$ Sodium Hydroxide & $\begin{array}{l}\text { Easily scratched after several } \\
\text { minutes }\end{array}$ & No additional damage \\
\hline $6 \mathrm{M}$ Sodium Hydroxide & $\begin{array}{l}\text { Easily scratched, ink/printing } \\
\text { destroyed }\end{array}$ & No additional damage \\
\hline $5 \%$ Hypochlorite (bleach) & $\begin{array}{l}\text { No discoloration or deformation, } \\
\text { no softening of adhesive }\end{array}$ & No change \\
\hline Concentrated Sulfuric Acid & $\begin{array}{l}\text { Yellow discoloration, ink } \\
\text { degradation, easily scratched }\end{array}$ & $\begin{array}{l}\text { Brownish-yellow } \\
\text { adhesive/surface, destroyed } \\
\text { where contacted by acid }\end{array}$ \\
\hline Concentrated Nitric Acid & $\begin{array}{l}\text { Light yellow discoloration, ink } \\
\text { destroyed, easily peeled from } \\
\text { surface }\end{array}$ & $\begin{array}{l}\text { Brownish yellow } \\
\text { adhesive/surface, destroyed } \\
\text { when contacted }\end{array}$ \\
\hline
\end{tabular}




\subsubsection{Weathering}

A prototype tamper tape (12/93) with a $2.54-\mathrm{cm}$-wide Confirm ${ }^{\circledR}$ window, PSA, and a polyester underlay was applied to four surfaces, i.e., bare, roughened aluminum; bare, roughened steel; smooth polyester fiberglass board; and Mil. Spec. polyurethane-painted steel panels. The 12/93 tamper tapes were exposed in Daytona Beach, Florida ( $90^{\circ}$ south) for 5 months, in the Weather-O-Meter (Xenon lamp) for.138 days, in the QUV cabinet (cycling exposure to ultraviolet light at $60^{\circ} \mathrm{C}$ and condensing humidity at $40^{\circ} \mathrm{C}$ ) for 85 days, and in the thermal cycling cabinet (cycling between $-18^{\circ} \mathrm{C}$ and $46^{\circ} \mathrm{C}$ ) for 161 days.

In addition to the studies with the $12 / 93$ tamper tapes, another $2.54-\mathrm{cm}$-wide Confirm ${ }^{\circledR}$ window prototype tamper tape similar to the 12/93 PSA tamper tape (except with a vinyl underlay) was tested later in the program. The exposures were the same as with the 12/93 prototype except the Weather-O-Meter exposures were not done, the Florida exposure lasted 2 months, and both the QUV cabinet and thermal cycling cabinet exposures lasted 56 days. Six surfaces were used with these tamper tapes, i.e., wood and Lexan polycarbonate in addition to the four listed above.

Visual evaluation/examination of the weathered tamper tapes was done at different time intervals by two or three persons who described the tamper tapes' appearance (with and without the $3 \mathrm{M}$ security illuminator) and evaluated the adhesion of the tamper tapes to the surface. A key to the numerical ranking codes used for assessing the weathered tamper tapes is given in Table 3.8.

Table 3.8. Numerical Ranking Code ${ }^{\mathrm{a}}$ for Assessing Tamper Tapes

\begin{tabular}{|c|c|c|}
\hline Appearance ${ }^{b}$ & Security Emblem ${ }^{\mathrm{c}}$ & Adhesived $^{d}$ \\
\hline $1=$ Poor & $1=$ Emblem not visible - area black & $1=>40 \%$ Debonding \\
\hline 2 = Fair & $2=$ Emblem barely visible-area grayish black & $2=26-40 \%$ debonding \\
\hline $3=$ Fair-Good & $3=$ Emblem grayish black and faded & $3=11-25 \%$ debonding \\
\hline $4=$ Good & $4=$ Emblem slightly faded & $4=3-10 \%$ debonding \\
\hline $5=$ Very Good & $5=$ Emblem had strong appearance & $5=2 \%$ or less debonding \\
\hline
\end{tabular}

a Refers to numbers used in Tables 3.9 to 3.15, and all Section 4.0 Tables

$\mathrm{b}$ Appearance was judged by a combination of inspections with and without the $3 \mathrm{M}$ illuminator. Some of the adhesive often attacked the Confirm ${ }^{\circledR}$, which in turn caused dark areas that were judged for how bad they appeared.

c Security emblem appearance was assessed with the $3 \mathrm{M}$ illuminator. Again, if the adhesive attacked the Confirm ${ }^{\circledR}$, the security emblems were damaged and the area appeared black or gray. In other instances the emblem was faded, barely visible, or not visible at all.

$\mathrm{d}$ Adhesion was based on an estimate of the amount of area that was debonded. 
Little or no change in visibility occurred with the tamper tapes' security features and the adhesion of the tamper tapes was excellent for the 12/93 tamper tapes exposed in Florida (5 months) and in the Weather-O-Meter (138 days). The results of the tamper tapes exposed in the Weather-O-Meter are given in Table 3.9. Under these conditions, the tamper tapes turned brownish yellow to light brown with those on the polyurethane paint looking the worst. For the Florida-exposed tamper tapes, there was only a slight change in appearance and in the visibility of the security features, but only on the polyester portion of the tamper tape (Table 3.10).

The greatest change in the 12/93 tamper tapes was observed in those from the QUV cabinet exposures. After 85 days of exposure, the security features were no longer visible on all surfaces (Table 3.11). On the steel and aluminum surfaces, adhesion in the window area was poor after 85 days of exposure. For the other two surfaces, adhesion remained good. All tamper tapes had some change in appearance.

The results of the 12/93 tamper tapes exposed to thermal cycling are given in Table 3.12. There was no change in the visibility of the security features or in adhesion, and there were only slight changes in appearance for the tamper tapes exposed to hot and cold for 161 days.

The results from the weathering of the PSA tamper tapes with vinyl underlay are given in Tables 3.13 through 3.15. After 2 months of exposure in Florida, there was no change in

Table 3.9. Results of Weather-O-Meter (Xenon Lamp) Exposure for 2.54-Centimeter-Wide Confirm ${ }^{\circledR}$ Window Tamper Tapes with Pressure Sensitive Adhesive and Polyester Underlay (12/93)

\begin{tabular}{lccccccc}
\hline & & \multicolumn{5}{c}{ Condition of Tamper Tapea } \\
\cline { 3 - 8 } & & \multicolumn{2}{c}{ Appearance } & Security & Emblem & \multicolumn{2}{c}{ Adhesion to Surface } \\
\cline { 3 - 8 } Surface & Days of & Window & Vinyl & Window & Vinyl & Window & \\
(Ident. No.) & Exposure & Area & Area & Area & Area & Area & Overall \\
\hline 1A Steel & 0 & 5 & 5 & 5 & 5 & 5 & 5 \\
(A1196) & 54 & 5 & 5 & 5 & 5 & 5 & 5 \\
& 68 & 3 & 4 & 5 & 5 & 5 & 5 \\
& 82 & 3 & 3 & 5 & 5 & 5 & 5 \\
& 96 & 3 & 3 & 5 & 5 & 5 & 5 \\
1B Steel & 138 & 3 & 3 & 5 & 5 & 5 & 5 \\
(A1193) & 0 & 5 & 5 & 5 & 5 & 5 & 5 \\
& 54 & 5 & 5 & 5 & 5 & 5 & 5 \\
& 68 & 4 & 4 & 5 & 5 & 5 & 5 \\
& 82 & 3 & 3 & 5 & 5 & 5 & 5 \\
& 96 & 3 & 3 & 5 & 5 & 5 & 5 \\
& 138 & 3 & 3 & 5 & 5 & 5 & 5 \\
\hline
\end{tabular}

a See Table 3.8 for a description of the numerical ranking code for assessing tamper tapes. 
Table 3.9. (Continued)

\begin{tabular}{|c|c|c|c|c|c|c|c|}
\hline \multirow[b]{3}{*}{$\begin{array}{l}\text { Surface } \\
\text { (Ident. No.) }\end{array}$} & \multirow[b]{3}{*}{$\begin{array}{l}\text { Days of } \\
\text { Exposure }\end{array}$} & \multicolumn{6}{|c|}{ Condition of Tamper Tape ${ }^{a}$} \\
\hline & & \multicolumn{2}{|c|}{ Appearance } & \multicolumn{2}{|c|}{ Security Emblem } & \multicolumn{2}{|c|}{ Adhesion to Surface } \\
\hline & & $\begin{array}{c}\text { Window } \\
\text { Area }\end{array}$ & $\begin{array}{l}\text { Vinyl } \\
\text { Area } \\
\end{array}$ & $\begin{array}{c}\text { Window } \\
\text { Area }\end{array}$ & $\begin{array}{l}\text { Vinyl } \\
\text { Area } \\
\end{array}$ & $\begin{array}{c}\text { Window } \\
\text { Area }\end{array}$ & Overall \\
\hline $\begin{array}{l}\text { 2A Aluminum } \\
\text { (A1195) }\end{array}$ & $\begin{array}{r}0 \\
68 \\
82 \\
96 \\
138\end{array}$ & $\begin{array}{c}5 \\
5 \\
4 \\
3-4 \\
3-4\end{array}$ & $\begin{array}{c}5 \\
5 \\
4 \\
3-4 \\
3-4\end{array}$ & $\begin{array}{l}5 \\
5 \\
5 \\
5 \\
5\end{array}$ & $\begin{array}{l}5 \\
5 \\
5 \\
5 \\
5\end{array}$ & $\begin{array}{l}5 \\
5 \\
5 \\
5 \\
5\end{array}$ & $\begin{array}{l}5 \\
5 \\
5 \\
5 \\
5\end{array}$ \\
\hline $\begin{array}{l}\text { 2B Aluminum } \\
\text { (A1194) }\end{array}$ & $\begin{array}{r}0 \\
68 \\
82 \\
96 \\
138\end{array}$ & $\begin{array}{c}5 \\
5 \\
4 \\
3-4 \\
3-4\end{array}$ & $\begin{array}{c}5 \\
5 \\
4 \\
3-4 \\
3-4\end{array}$ & $\begin{array}{l}5 \\
5 \\
5 \\
5 \\
5\end{array}$ & $\begin{array}{l}5 \\
5 \\
5 \\
5 \\
5\end{array}$ & $\begin{array}{l}5 \\
5 \\
5 \\
5 \\
5\end{array}$ & $\begin{array}{l}5 \\
5 \\
5 \\
5 \\
5\end{array}$ \\
\hline $\begin{array}{l}\text { 3A Mil. Spec. } \\
\text { Polyurethane } \\
\text { Painted Steel } \\
\text { (A11097) }\end{array}$ & $\begin{array}{r}0 \\
28 \\
44 \\
68 \\
82 \\
96 \\
138\end{array}$ & $\begin{array}{l}5 \\
5 \\
4 \\
4 \\
3 \\
3 \\
3\end{array}$ & $\begin{array}{l}5 \\
5 \\
5 \\
4 \\
3 \\
3 \\
3\end{array}$ & $\begin{array}{l}5 \\
5 \\
5 \\
5 \\
5 \\
5 \\
5\end{array}$ & $\begin{array}{l}5 \\
5 \\
5 \\
5 \\
5 \\
5 \\
5\end{array}$ & $\begin{array}{l}5 \\
5 \\
5 \\
5 \\
5 \\
5 \\
5\end{array}$ & $\begin{array}{l}5 \\
5 \\
5 \\
5 \\
5 \\
5 \\
5\end{array}$ \\
\hline $\begin{array}{l}\text { 3B Mil. Spec. } \\
\text { Polyurethane } \\
\text { Painted Steel } \\
\text { (A1200) }\end{array}$ & $\begin{array}{r}0 \\
28 \\
\cdot \quad 44 \\
54 \\
68 \\
82 \\
96 \\
138\end{array}$ & $\begin{array}{c}5 \\
5 \\
4 \\
4 \\
3 \\
2-3 \\
2-3 \\
2-3\end{array}$ & $\begin{array}{c}5 \\
5 \\
5 \\
4 \\
4 \\
3 \\
3 \\
2-3\end{array}$ & $\begin{array}{l}5 \\
5 \\
5 \\
5 \\
5 \\
5 \\
5 \\
5\end{array}$ & $\begin{array}{l}5 \\
5 \\
5 \\
5 \\
5 \\
5 \\
5 \\
5\end{array}$ & $\begin{array}{l}5 \\
5 \\
5 \\
5 \\
5 \\
5 \\
5 \\
5\end{array}$ & $\begin{array}{l}5 \\
5 \\
5 \\
5 \\
5 \\
5 \\
5 \\
5\end{array}$ \\
\hline $\begin{array}{l}\text { 4A Fiberglass } \\
\text { Board (A1198) }\end{array}$ & $\begin{array}{r}0 \\
28 \\
44 \\
54 \\
68 \\
82 \\
96 \\
138\end{array}$ & $\begin{array}{c}5 \\
5 \\
4-5 \\
3-4 \\
3 \\
3 \\
3 \\
3\end{array}$ & $\begin{array}{c}5 \\
5 \\
4-5 \\
4 \\
4 \\
3-4 \\
3-4 \\
3\end{array}$ & $\begin{array}{l}5 \\
5 \\
5 \\
5 \\
5 \\
5 \\
5 \\
5\end{array}$ & $\begin{array}{l}5 \\
5 \\
5 \\
5 \\
5 \\
5 \\
5 \\
5\end{array}$ & $\begin{array}{l}5 \\
5 \\
5 \\
5 \\
5 \\
5 \\
5 \\
5\end{array}$ & $\begin{array}{l}5 \\
5 \\
5 \\
5 \\
5 \\
5 \\
5 \\
5\end{array}$ \\
\hline $\begin{array}{l}\text { 4B Fiberglass } \\
\text { Board (A1199) }\end{array}$ & $\begin{array}{r}0 \\
28 \\
44 \\
54 \\
68 \\
82 \\
96 \\
138\end{array}$ & $\begin{array}{c}5 \\
5 \\
4-5 \\
3-4 \\
3 \\
3 \\
3 \\
3\end{array}$ & $\begin{array}{c}5 \\
5 \\
4-5 \\
4 \\
4 \\
3-4 \\
3-4 \\
3\end{array}$ & $\begin{array}{l}5 \\
5 \\
5 \\
5 \\
5 \\
5 \\
.5 \\
5\end{array}$ & $\begin{array}{l}5 \\
5 \\
5 \\
5 \\
5 \\
5 \\
5 \\
5\end{array}$ & $\begin{array}{l}5 \\
5 \\
5 \\
5 \\
5 \\
5 \\
5 \\
5\end{array}$ & $\begin{array}{l}5 \\
5 \\
5 \\
5 \\
5 \\
5 \\
5 \\
5\end{array}$ \\
\hline
\end{tabular}

a See Table 3.8 for a description of the numerical ranking code for assessing tamper tapes 
Table 3.10. Results of Daytona Beach, Florida Exposure for 2.54-Centimeter-Wide Confirm $^{\circledR}$ Window Tamper Tapes with Pressure Sensitive Adhesive and Polyester Underlay (12/93)

\begin{tabular}{|c|c|c|c|c|c|c|c|}
\hline \multirow[b]{3}{*}{$\begin{array}{l}\text { Surface } \\
\text { (Ident. No.) }\end{array}$} & \multirow[b]{3}{*}{$\begin{array}{l}\text { Months } \\
\text { Exposure }\end{array}$} & \multicolumn{6}{|c|}{ Condition of Tamper Tape ${ }^{a}$} \\
\hline & & \multicolumn{2}{|c|}{ Appearance } & \multicolumn{2}{|c|}{ Security Emblem } & \multicolumn{2}{|c|}{ Adhesion to Surface } \\
\hline & & $\begin{array}{c}\text { Window } \\
\text { Area } \\
\end{array}$ & $\begin{array}{l}\text { Vinyl } \\
\text { Area } \\
\end{array}$ & $\begin{array}{c}\text { Window } \\
\text { Area } \\
\end{array}$ & $\begin{array}{l}\text { Vinyl } \\
\text { Area } \\
\end{array}$ & $\begin{array}{c}\text { Window } \\
\text { Area }\end{array}$ & Overall \\
\hline Steel (A1189) & $\begin{array}{l}0 \\
1 \\
2 \\
3 \\
4 \\
5\end{array}$ & $\begin{array}{c}4-5 \\
4 \\
4 \\
4 \\
4 \\
4\end{array}$ & $\begin{array}{l}5 \\
4 \\
4 \\
4 \\
4 \\
4\end{array}$ & $\begin{array}{l}5 \\
-b \\
- \\
5 \\
- \\
5\end{array}$ & $\begin{array}{l}5 \\
- \\
- \\
5 \\
- \\
4\end{array}$ & $\begin{array}{l}5 \\
5 \\
5 \\
5 \\
5 \\
5\end{array}$ & $\begin{array}{l}5 \\
5 \\
5 \\
5 \\
5 \\
5\end{array}$ \\
\hline Steel (A1190) & $\begin{array}{l}0 \\
1 \\
2 \\
3 \\
4 \\
5\end{array}$ & $\begin{array}{c}3 \\
2-3 \\
2-3 \\
2-3 \\
2-3 \\
2-3\end{array}$ & $\begin{array}{c}4-5 \\
4 \\
4 \\
4 \\
4 \\
4\end{array}$ & $\begin{array}{l}5 \\
- \\
- \\
5 \\
- \\
5\end{array}$ & $\begin{array}{l}5 \\
- \\
- \\
5 \\
- \\
4\end{array}$ & $\begin{array}{l}5 \\
5 \\
5 \\
5 \\
5 \\
5\end{array}$ & $\begin{array}{l}5 \\
5 \\
5 \\
5 \\
5 \\
5\end{array}$ \\
\hline $\begin{array}{l}\text { Aluminum } \\
\text { (A1186) }\end{array}$ & $\begin{array}{l}0 \\
1 \\
2 \\
3 \\
4 \\
5\end{array}$ & $\begin{array}{l}4-5 \\
4-5 \\
4-5 \\
4-5 \\
4-5 \\
4-5\end{array}$ & $\begin{array}{l}4-5 \\
4-5 \\
4-5 \\
4-5 \\
4-5 \\
4-5\end{array}$ & $\begin{array}{l}5 \\
- \\
- \\
5 \\
- \\
4\end{array}$ & $\begin{array}{l}5 \\
- \\
- \\
5 \\
- \\
4\end{array}$ & $\begin{array}{l}5 \\
5 \\
5 \\
5 \\
5 \\
5\end{array}$ & $\begin{array}{l}5 \\
5 \\
5 \\
5 \\
5 \\
5\end{array}$ \\
\hline $\begin{array}{l}\text { Aluminum } \\
\text { (A 1187) }\end{array}$ & $\begin{array}{l}0 \\
1 \\
2 \\
3 \\
4 \\
5\end{array}$ & $\begin{array}{l}4-5 \\
4-5 \\
4-5 \\
4-5 \\
4-5 \\
4-5\end{array}$ & $\begin{array}{c}5 \\
5 \\
5 \\
5 \\
5 \\
4-5\end{array}$ & $\begin{array}{l}5 \\
- \\
5 \\
5 \\
- \\
4\end{array}$ & $\begin{array}{l}5 \\
- \\
- \\
5 \\
- \\
4\end{array}$ & $\begin{array}{l}5 \\
5 \\
5 \\
5 \\
5 \\
5\end{array}$ & $\begin{array}{l}5 \\
5 \\
5 \\
5 \\
5 \\
5\end{array}$ \\
\hline $\begin{array}{l}\text { Mil. Spec. } \\
\text { Polyurethane } \\
\text { Painted } \\
\text { Steel (A1185) }\end{array}$ & $\begin{array}{l}0 \\
1 \\
2 \\
3 \\
4 \\
5\end{array}$ & $\begin{array}{l}4-5 \\
4-5 \\
4-5 \\
4-5 \\
4-5 \\
4-5\end{array}$ & $\begin{array}{c}5 \\
5 \\
5 \\
4-5 \\
4-5 \\
4-5\end{array}$ & $\begin{array}{l}5 \\
- \\
- \\
5 \\
- \\
5\end{array}$ & $\begin{array}{l}5 \\
- \\
- \\
5 \\
- \\
4\end{array}$ & $\begin{array}{l}5 \\
5 \\
5 \\
5 \\
5 \\
5\end{array}$ & $\begin{array}{l}5 \\
5 \\
5 \\
5 \\
5 \\
5\end{array}$ \\
\hline $\begin{array}{l}\text { Mil. Spec. } \\
\text { Polyurethane } \\
\text { Painted } \\
\text { Steel (A1188) }\end{array}$ & $\begin{array}{l}0 \\
1 \\
2 \\
3 \\
4 \\
5\end{array}$ & $\begin{array}{l}4-5 \\
4-5 \\
4-5 \\
4-5 \\
4-5 \\
4-5\end{array}$ & $\begin{array}{c}5 \\
5 \\
5 \\
4-5 \\
4-5 \\
4-5\end{array}$ & $\begin{array}{l}5 \\
- \\
- \\
5 \\
- \\
5\end{array}$ & $\begin{array}{l}5 \\
- \\
- \\
5 \\
- \\
4\end{array}$ & $\begin{array}{l}5 \\
5 \\
5 \\
5 \\
5 \\
5\end{array}$ & $\begin{array}{l}5 \\
5 \\
5 \\
5 \\
5 \\
5\end{array}$ \\
\hline
\end{tabular}

a See Table 3.8 for a description of the numerical ranking code for assessing tamper tapes.

b No data 
Table 3.10. (Continued)

\begin{tabular}{|c|c|c|c|c|c|c|c|}
\hline \multirow[b]{3}{*}{$\begin{array}{l}\text { Surfiace } \\
\text { (Ident. No.) }\end{array}$} & \multirow[b]{3}{*}{$\begin{array}{c}\text { Months } \\
\text { Exposure }\end{array}$} & \multicolumn{6}{|c|}{ Condition of Tamper Tape ${ }^{a}$} \\
\hline & & \multicolumn{2}{|c|}{ Appearance } & \multicolumn{2}{|c|}{ Security Emblem } & \multicolumn{2}{|c|}{ Adhesion to Surface } \\
\hline & & $\begin{array}{c}\text { Window } \\
\text { Area }\end{array}$ & $\begin{array}{l}\text { Vinyl } \\
\text { Area }\end{array}$ & $\begin{array}{l}\text { Window } \\
\text { Area } \\
\end{array}$ & $\begin{array}{l}\text { Vinyl } \\
\text { Area }\end{array}$ & $\begin{array}{c}\text { Window } \\
\text { Area }\end{array}$ & Overall \\
\hline $\begin{array}{l}\text { Polyester } \\
\text { Fiberglass } \\
\text { Board } \\
\text { (A1191) }\end{array}$ & $\begin{array}{l}0 \\
1 \\
2 \\
3 \\
4 \\
5\end{array}$ & $\begin{array}{c}5 \\
5 \\
5 \\
5 \\
5 \\
4-5\end{array}$ & $\begin{array}{c}5 \\
5 \\
5 \\
5 \\
5 \\
4-5\end{array}$ & $\begin{array}{l}5 \\
-b \\
- \\
5 \\
- \\
5\end{array}$ & $\begin{array}{l}5 \\
- \\
- \\
5 \\
5\end{array}$ & $\begin{array}{l}5 \\
5 \\
5 \\
5 \\
5 \\
5\end{array}$ & $\begin{array}{l}5 \\
5 \\
5 \\
5 \\
5 \\
5\end{array}$ \\
\hline $\begin{array}{l}\text { Polyester } \\
\text { Fiberglass } \\
\text { Board } \\
\text { (A1192) }\end{array}$ & $\begin{array}{l}0 \\
1 \\
2 \\
3 \\
4 \\
5\end{array}$ & $\begin{array}{c}5 \\
5 \\
5 \\
5 \\
5 \\
4-5\end{array}$ & $\begin{array}{c}5 \\
5 \\
5 \\
5 \\
5 \\
4-5\end{array}$ & $\begin{array}{l}5 \\
- \\
- \\
5 \\
- \\
5\end{array}$ & $\begin{array}{l}5 \\
- \\
5 \\
5 \\
4\end{array}$ & $\begin{array}{l}5 \\
5 \\
5 \\
5 \\
5 \\
5\end{array}$ & $\begin{array}{l}5 \\
5 \\
5 \\
5 \\
5 \\
5\end{array}$ \\
\hline
\end{tabular}

a See Table 3.8 for a description of the numerical ranking code for assessing tamper tapes.

b No data

Table 3.11. Results of QUV Cabinet Exposure-for 2.54-Centimeter-Wide Confirm ${ }^{\circledR}$ Window Tamper Tapes with Pressure Sensitive Adhesive and Polyester Underlay (12/93)

\begin{tabular}{|c|c|c|c|c|c|c|c|}
\hline \multirow[b]{3}{*}{ Surface } & \multirow[b]{3}{*}{$\begin{array}{c}\text { Days of } \\
\text { Exposure }\end{array}$} & \multicolumn{6}{|c|}{ Condition of Tamper Tape } \\
\hline & & \multicolumn{2}{|c|}{ Appearance } & \multicolumn{2}{|c|}{ Security Emblem } & \multicolumn{2}{|c|}{ Adhesion to Surface } \\
\hline & & $\begin{array}{c}\text { Window } \\
\text { Area }\end{array}$ & $\begin{array}{l}\text { Vinyl } \\
\text { Area }\end{array}$ & $\begin{array}{c}\text { Window } \\
\text { Area }\end{array}$ & $\begin{array}{l}\text { Vinyl } \\
\text { Area }\end{array}$ & $\begin{array}{l}\text { Window } \\
\text { Area }\end{array}$ & Overall \\
\hline Steel & $\begin{array}{r}0 \\
7 \\
14 \\
21 \\
28 \\
42 \\
56 \\
70 \\
85 \\
91 \\
100\end{array}$ & $\begin{array}{c}5 \\
5 \\
4-5 \\
4 \\
4 \\
3-4 \\
3 \\
3 \\
3 \\
2 \\
2\end{array}$ & $\begin{array}{c}5 \\
5 \\
5 \\
5 \\
4-5 \\
4 \\
3 \\
3 \\
3 \\
2 \\
2\end{array}$ & $\begin{array}{c}5 \\
5 \\
5 \\
5 \\
5 \\
5 \\
3-4 \\
3 \\
1 \\
1 \\
1\end{array}$ & $\begin{array}{l}5 \\
5 \\
5 \\
5 \\
5 \\
5 \\
4 \\
3 \\
1 \\
1 \\
1\end{array}$ & $\begin{array}{l}5 \\
5 \\
5 \\
5 \\
5 \\
4 \\
3 \\
3 \\
2 \\
2 \\
1\end{array}$ & $\begin{array}{c}5 \\
5 \\
5 \\
5 \\
5 \\
4-5 \\
4 \\
4 \\
3 \\
2 \\
1\end{array}$ \\
\hline Aluminum & $\begin{array}{r}0 \\
7 \\
14 \\
21 \\
28 \\
42 \\
56 \\
70 \\
85 \\
91 \\
100\end{array}$ & $\begin{array}{c}5 \\
5 \\
4 \\
3-4 \\
3-4 \\
3 \\
2 \\
2 \\
2 \\
2 \\
2\end{array}$ & $\begin{array}{c}5 \\
5 \\
5 \\
4-5 \\
4-5 \\
4 \\
3 \\
3 \\
3 \\
2 \\
2\end{array}$ & $\begin{array}{l}5 \\
5 \\
5 \\
5 \\
5 \\
5 \\
4 \\
3 \\
1 \\
1 \\
1\end{array}$ & $\begin{array}{l}5 \\
5 \\
5 \\
5 \\
5 \\
5 \\
4 \\
3 \\
1 \\
1 \\
1\end{array}$ & $\begin{array}{c}5 \\
5 \\
5 \\
5 \\
4 \\
3-4 \\
3 \\
2-3 \\
2 \\
1-2 \\
1-2\end{array}$ & $\begin{array}{c}5 \\
5 \\
5 \\
5 \\
4-5 \\
4-5 \\
4 \\
3 \\
2-3 \\
2 \\
2\end{array}$ \\
\hline
\end{tabular}

a See Table 3.8 for a description of the numerical ranking code for assessing tamper tapes. 
Table 3.11. (Continued)

\begin{tabular}{|c|c|c|c|c|c|c|c|}
\hline \multirow[b]{3}{*}{ Surface } & \multirow[b]{3}{*}{$\begin{array}{l}\text { Days of } \\
\text { Exposure }\end{array}$} & \multicolumn{6}{|c|}{ Condition of Tamper Tape ${ }^{a}$} \\
\hline & & \multicolumn{2}{|c|}{ Appearance } & \multicolumn{2}{|c|}{ Security Emblem } & \multicolumn{2}{|c|}{ Adhesion to Surface } \\
\hline & & $\begin{array}{c}\text { Window } \\
\text { Area } \\
\end{array}$ & $\begin{array}{l}\text { Vinyl } \\
\text { Area }\end{array}$ & $\begin{array}{c}\text { Window } \\
\text { Area }\end{array}$ & $\begin{array}{l}\text { Vinyl } \\
\text { Area } \\
\end{array}$ & $\begin{array}{c}\text { Window } \\
\text { Area }\end{array}$ & Overall \\
\hline $\begin{array}{l}\text { Mil. Spec. } \\
\text { Polyurethane } \\
\text { Painted } \\
\text { Steel }\end{array}$ & $\begin{array}{r}0 \\
7 \\
14 \\
21 \\
28 \\
42 \\
56 \\
70 \\
85 \\
91 \\
100\end{array}$ & $\begin{array}{c}5 \\
5 \\
5 \\
5 \\
4-5 \\
4-5 \\
3 \\
3 \\
2 \\
2 \\
2\end{array}$ & $\begin{array}{c}5 \\
5 \\
5 \\
5 \\
4-5 \\
4-5 \\
4 \\
3-4 \\
3 \\
3 \\
3\end{array}$ & $\begin{array}{l}5 \\
5 \\
5 \\
5 \\
5 \\
5 \\
4 \\
3 \\
1 \\
1 \\
1\end{array}$ & $\begin{array}{l}5 \\
5 \\
5 \\
5 \\
5 \\
5 \\
4 \\
3 \\
1 \\
1 \\
1\end{array}$ & $\begin{array}{l}5 \\
5 \\
5 \\
5 \\
5 \\
5 \\
5 \\
5 \\
5 \\
5 \\
5\end{array}$ & $\begin{array}{l}5 \\
5 \\
5 \\
5 \\
5 \\
5 \\
5 \\
5 \\
5 \\
5 \\
5\end{array}$ \\
\hline $\begin{array}{l}\text { Polyester } \\
\text { Fiberglass } \\
\text { Board }\end{array}$ & $\begin{array}{r}0 \\
7 \\
14 \\
21 \\
28 \\
42 \\
56 \\
70 \\
85 \\
91 \\
100\end{array}$ & $\begin{array}{c}5 \\
5 \\
5 \\
5 \\
4-5 \\
4-5 \\
4 \\
3 \\
2-3 \\
2-3 \\
2-3\end{array}$ & $\begin{array}{c}5 \\
5 \\
5 \\
5 \\
4-5 \\
4-5 \\
4 \\
3-4 \\
3 \\
3 \\
3\end{array}$ & $\begin{array}{l}5 \\
5 \\
5 \\
5 \\
5 \\
5 \\
3 \\
3 \\
1 \\
1 \\
1\end{array}$ & $\begin{array}{l}5 \\
5 \\
5 \\
5 \\
5 \\
5 \\
3 \\
3 \\
1 \\
1 \\
1\end{array}$ & $\begin{array}{l}5 \\
5 \\
5 \\
5 \\
5 \\
5 \\
5 \\
5 \\
5 \\
5 \\
5\end{array}$ & $\begin{array}{l}5 \\
5 \\
5 \\
5 \\
5 \\
5 \\
5 \\
5 \\
5 \\
5 \\
5\end{array}$ \\
\hline
\end{tabular}

a See Table 3.8 for a description of the numerical ranking code for assessing tamper tapes.

visibility of the security features and no loss of adhesion, with only slight changes in appearance (Table 3.13). For the tamper tapes exposed in the QUV cabinet, there was no loss in adhesion after 56 days on any of the surfaces. After 21 to 42 days, there was a slight change in the visibility of the security features, increasing to a moderate change after 49 days (Table 3.14). Usually, only the vinyl portion of the tamper tape was affected. After 56 days, the tamper tape bonded to wood had the worst appearance, probably because of the weathered wood showing through the tamper tape. For the tamper tapes exposed in the thermal cycling cabinet, there were essentially no changes noted after 56 days (Table 3.15).

To compare the prototype $2.54-\mathrm{cm}$-wide Confirm $^{\circledR}$ window tamper tapes with polyester underlay (12/93) to those with vinyl underlay, weathering periods of 2 months in Florida and 56 days in the QUV and thermal cycling cabinet must be used. (The PSA tamper tape with the 
Table 3.12. Results of Thermal Cycling Exposure $\left(-54^{\circ} \mathrm{C}\right.$ to $\left.46^{\circ} \mathrm{C}\right)$ for 2.54 -Centimeter-Wide Confirm ${ }^{\circledR}$ Window Tamper Tapes with Pressure Sensitive Adhesive and Polyester Underlay (12/93)

\begin{tabular}{|c|c|c|c|c|c|c|c|}
\hline \multirow[b]{3}{*}{ Surface. } & \multirow[b]{3}{*}{$\begin{array}{c}\text { Days of } \\
\text { Exposure }\end{array}$} & \multicolumn{6}{|c|}{ Condition of Tamper Tape ${ }^{a}$} \\
\hline & & \multicolumn{2}{|c|}{ Appearance } & \multicolumn{2}{|c|}{ Security Emblem } & \multicolumn{2}{|c|}{ Adhesion to Surface } \\
\hline & & $\begin{array}{c}\text { Window } \\
\text { Area }\end{array}$ & $\begin{array}{l}\text { Vinyl } \\
\text { Area } \\
\end{array}$ & $\begin{array}{c}\text { Window } \\
\text { Area }\end{array}$ & $\begin{array}{l}\text { Vinyl } \\
\text { Area } \\
\end{array}$ & $\begin{array}{c}\text { Window } \\
\text { Area }\end{array}$ & Overall \\
\hline Steel & $\begin{array}{r}0 \\
28 \\
42 \\
56 \\
112 \\
126\end{array}$ & $\begin{array}{c}5 \\
5 \\
4-5 \\
4-5 \\
4-5 \\
4-5\end{array}$ & $\begin{array}{c}5 \\
5 \\
4-5 \\
4-5 \\
4-5 \\
4-5\end{array}$ & $\begin{array}{l}5 \\
5 \\
5 \\
5 \\
5 \\
5\end{array}$ & $\begin{array}{l}5 \\
5 \\
5 \\
5 \\
5 \\
5\end{array}$ & $\begin{array}{r}5 \\
5 \\
5 \\
5 \\
5 \\
5\end{array}$ & $\begin{array}{l}5 \\
5 \\
5 \\
5 \\
5 \\
5\end{array}$ \\
\hline Aluminum & $\begin{array}{r}0 \\
28 \\
42 \\
112 \\
126\end{array}$ & $\begin{array}{c}5 \\
5 \\
4-5 \\
4-5 \\
4-5\end{array}$ & $\begin{array}{c}5 \\
5 \\
4-5 \\
4-5 \\
4-5\end{array}$ & $\begin{array}{l}5 \\
5 \\
5 \\
5 \\
5\end{array}$ & $\begin{array}{l}5 \\
5 \\
5 \\
5 \\
5\end{array}$ & $\begin{array}{l}5 \\
5 \\
5 \\
5 \\
5\end{array}$ & $\begin{array}{l}5 \\
5 \\
5 \\
5 \\
5\end{array}$ \\
\hline $\begin{array}{l}\text { Mil. Spec. } \\
\text { Polyurethane } \\
\text { Painted Steel }\end{array}$ & $\begin{array}{r}0 \\
28 \\
42 \\
56 \\
112 \\
126\end{array}$ & $\begin{array}{c}5 \\
5 \\
4-5 \\
4-5 \\
4-5 \\
4-5\end{array}$ & $\begin{array}{c}5 \\
5 \\
4-5 \\
4-5 \\
4-5 \\
4-5\end{array}$ & $\begin{array}{l}5 \\
5 \\
5 \\
5 \\
5 \\
5\end{array}$ & $\begin{array}{l}5 \\
5 \\
5 \\
5 \\
5 \\
5\end{array}$ & $\begin{array}{c}5 \\
5 \\
5 \\
5 \\
5 \\
5^{b}\end{array}$ & $\begin{array}{c}5 \\
5 \\
5 \\
5 \\
5 \\
5^{b}\end{array}$ \\
\hline $\begin{array}{l}\text { Polyester } \\
\text { Fiberglass } \\
\text { Board }\end{array}$ & $\begin{array}{r}0 \\
28 \\
42 \\
112 \\
126\end{array}$ & $\begin{array}{c}5 \\
5 \\
4-5 \\
4-5 \\
4-5\end{array}$ & $\begin{array}{c}5 \\
5 \\
4-5 \\
4-5 \\
4-5\end{array}$ & $\begin{array}{l}5 \\
5 \\
5 \\
5 \\
5\end{array}$ & $\begin{array}{l}5 \\
5 \\
5 \\
5 \\
5\end{array}$ & $\begin{array}{l}5 \\
5 \\
5 \\
5 \\
5\end{array}$ & $\begin{array}{l}5 \\
5 \\
5 \\
5 \\
5\end{array}$ \\
\hline
\end{tabular}

a See Table 3.8 for a description of the numerical ranking code for assessing tamper tapes.

b Adhesion of polyurethane paint to which tamper tape was adhered was peeling badly, therefore, it was difficult to determine if there was a loss of adhesion.

2.54-cm-wide Confirm ${ }^{\circledR}$ window and vinyl underlay was tested for only 2 months in Florida and only 56 days in the QUV and thermal cycling cabinets, whereas the 12/93 tamper tape was tested for longer periods of time.) After 2 months in Daytona Beach, Florida, there was no loss of adhesion and no change in the visibility of the security features on any of the tamper tapes. They did yellow somewhat in that period of time. In the QUV cabinet, the two types of tamper tapes performed similarly in terms of visibility of the security features; they were within the slight-tomoderate change range. The 12/93 tamper tapes had some loss of adhesion in the window area on steel and aluminum compared to the vinyl underlay tamper tapes. In addition, the vinyl underlay tamper tapes had a slightly better appearance. Essentially no changes to any of the tamper tapes occurred in the thermal cycling cabinet. 
Table 3.13. Results of Daytona Beach, Florida Exposure for 2.54-Centimeter-Wide Confirm ${ }^{\circledR}$ Window Tamper Tapes with Pressure Sensitive Adhesive and Vinyl Underlay (Number and Bar Code, ATM Oceanfront $90^{\circ}$ South)

\begin{tabular}{|c|c|c|c|c|c|c|c|}
\hline \multirow[b]{3}{*}{ Surface } & \multirow[b]{3}{*}{$\begin{array}{c}\text { Months } \\
\text { Exposure }\end{array}$} & \multicolumn{6}{|c|}{ Condition of Tamper Tape ${ }^{a}$} \\
\hline & & \multicolumn{2}{|c|}{ Appearance } & \multicolumn{2}{|c|}{ Security Emblem } & \multicolumn{2}{|c|}{ Adhesion to Surface } \\
\hline & & $\begin{array}{c}\text { Window } \\
\text { Area }\end{array}$ & $\begin{array}{l}\text { Vinyl } \\
\text { Area } \\
\end{array}$ & $\begin{array}{c}\text { Window } \\
\text { Area }\end{array}$ & $\begin{array}{l}\text { Vinyl } \\
\text { Area } \\
\end{array}$ & $\begin{array}{c}\text { Window } \\
\text { Area }\end{array}$ & Overall \\
\hline Steel & $\begin{array}{l}1 \\
2\end{array}$ & $\begin{array}{l}4-5 \\
4-5\end{array}$ & $\begin{array}{l}5 \\
5\end{array}$ & $\begin{array}{l}-b \\
5\end{array}$ & 5 & $\begin{array}{l}5 \\
5\end{array}$ & $\begin{array}{l}5 \\
5\end{array}$ \\
\hline Aluminum & $\frac{1}{2}$ & $\begin{array}{l}4-5 \\
4-5\end{array}$ & $\begin{array}{l}5 \\
5\end{array}$ & $\overline{5}$ & 5 & $\begin{array}{l}5 \\
5\end{array}$ & $\begin{array}{l}5 \\
5\end{array}$ \\
\hline Wood & $\frac{1}{2}$ & $\begin{array}{l}4-5 \\
4-5\end{array}$ & $\begin{array}{c}5 \\
4-5\end{array}$ & $\overline{4}$ & $\overline{4}$ & $\begin{array}{l}5 \\
5\end{array}$ & $\begin{array}{l}5 \\
5\end{array}$ \\
\hline $\begin{array}{l}\text { Polyester } \\
\text { Fiberglass } \\
\text { Board }\end{array}$ & $\begin{array}{l}1 \\
2\end{array}$ & $\begin{array}{l}4-5 \\
4-5\end{array}$ & $\begin{array}{l}5 \\
5\end{array}$ & 5 & 5 & $\begin{array}{l}5 \\
5\end{array}$ & $\begin{array}{l}5 \\
5\end{array}$ \\
\hline $\begin{array}{l}\text { Lexan } \\
\text { Polycarbonate }\end{array}$ & $\begin{array}{l}1 \\
2\end{array}$ & $\begin{array}{l}4-5 \\
4-5\end{array}$ & $\begin{array}{l}5 \\
5\end{array}$ & 5 & 5 & $\begin{array}{l}5 \\
5\end{array}$ & $\begin{array}{l}5 \\
5\end{array}$ \\
\hline $\begin{array}{l}\text { Mil. Spec. } \\
\text { Polyurethanc } \\
\text { Painted Steel }\end{array}$ & $\begin{array}{l}1 \\
2\end{array}$ & $\begin{array}{l}4-5 \\
4-5\end{array}$ & $\begin{array}{l}5 \\
5\end{array}$ & $\overline{4}$ & 4 & $\begin{array}{l}5 \\
5\end{array}$ & $\begin{array}{l}5 \\
5\end{array}$ \\
\hline
\end{tabular}

a See Table 3.8 for a description of the numerical ranking code for assessing tamper tapes.

b No data

Table 3.14. Results of QUV Cabinet Exposure for 2.54-Centimeter-Wide Confirm ${ }^{\circledR}$ Window Tamper Tapes with Pressure Sensitive Adhesive and Vinyl Underlay (Number and Bar Code)

\begin{tabular}{|c|c|c|c|c|c|c|c|}
\hline \multirow[b]{3}{*}{ Surface } & \multirow[b]{3}{*}{$\begin{array}{l}\text { Days of } \\
\text { Exposure }\end{array}$} & \multicolumn{6}{|c|}{ Condition of Tamper Tape ${ }^{a}$} \\
\hline & & \multicolumn{2}{|c|}{ Appearance } & \multicolumn{2}{|c|}{ Security Emblem } & \multicolumn{2}{|c|}{ Adhesion to Surface } \\
\hline & & $\begin{array}{c}\text { Window } \\
\text { Area }\end{array}$ & Vinyl Area & $\begin{array}{c}\text { Window } \\
\text { Area }\end{array}$ & Vinyl Area & $\begin{array}{c}\text { Window } \\
\text { Area } \\
\end{array}$ & Overall \\
\hline Stcel & $\begin{array}{r}0 \\
7 \\
14 \\
21 \\
28 \\
35 \\
42 \\
49 \\
56\end{array}$ & $\begin{array}{l}4 \\
3 \\
3 \\
3 \\
3 \\
3 \\
3 \\
3 \\
3\end{array}$ & $\begin{array}{l}5 \\
5 \\
5 \\
5 \\
5 \\
5 \\
5 \\
5 \\
4\end{array}$ & $\begin{array}{l}5 \\
5 \\
5 \\
5 \\
5 \\
5 \\
5 \\
4 \\
4\end{array}$ & $\begin{array}{l}5 \\
5 \\
5 \\
5 \\
5 \\
5 \\
4 \\
3 \\
3\end{array}$ & $\begin{array}{l}5 \\
5 \\
5 \\
5 \\
5 \\
5 \\
5 \\
5 \\
5\end{array}$ & $\begin{array}{l}5 \\
5 \\
5 \\
5 \\
5 \\
5 \\
5 \\
5 \\
5\end{array}$ \\
\hline
\end{tabular}

\footnotetext{
a See Table 3.8 for a description of the numerical ranking code for assessing tamper tapes.
} 
Table 3.14. (Continued)

\begin{tabular}{|c|c|c|c|c|c|c|c|}
\hline \multirow[b]{3}{*}{ Surface } & \multirow[b]{3}{*}{$\begin{array}{l}\text { Days of } \\
\text { Exposure }\end{array}$} & \multicolumn{6}{|c|}{ Condition of Tamper Tape ${ }^{\mathrm{a}}$} \\
\hline & & \multicolumn{2}{|c|}{ Appearance } & \multicolumn{2}{|c|}{ Security Emblem } & \multicolumn{2}{|c|}{ Adhesion to Surface } \\
\hline & & $\begin{array}{c}\text { Window } \\
\text { Area }\end{array}$ & Vinyl Area & $\begin{array}{c}\text { Window } \\
\text { Area }\end{array}$ & Vinyl Area & $\begin{array}{c}\text { Window } \\
\text { Area }\end{array}$ & Overall \\
\hline Aluminum & $\begin{array}{r}0 \\
7 \\
14 \\
21 \\
28 \\
35 \\
42 \\
49 \\
56\end{array}$ & $\begin{array}{l}4 \\
4 \\
4 \\
4 \\
4 \\
4 \\
4 \\
4 \\
4\end{array}$. & $\begin{array}{l}5 \\
5 \\
5 \\
4 \\
4 \\
4 \\
4 \\
4 \\
4\end{array}$ & $\begin{array}{l}5 \\
5 \\
5 \\
4 \\
4 \\
4 \\
4 \\
4 \\
4\end{array}$ & $\begin{array}{l}5 \\
5 \\
5 \\
4 \\
4 \\
4 \\
4 \\
4 \\
4\end{array}$ & $\begin{array}{l}5 \\
5 \\
5 \\
5 \\
5 \\
5 \\
5 \\
5 \\
5\end{array}$ & $\begin{array}{l}5 \\
5 \\
5 \\
5 \\
5 \\
5 \\
5 \\
5 \\
5\end{array}$ \\
\hline Wood & $\begin{array}{r}0 \\
7 \\
14 \\
21 \\
28 \\
35 \\
42 \\
49 \\
56\end{array}$ & $\begin{array}{l}4 \\
3 \\
2 \\
2 \\
2 \\
2 \\
2 \\
2 \\
2\end{array}$ & $\begin{array}{l}5 \\
5 \\
5 \\
5 \\
5 \\
5 \\
5 \\
4 \\
3\end{array}$ & $\begin{array}{l}5 \\
5 \\
5 \\
4 \\
4 \\
4 \\
4 \\
4 \\
3\end{array}$ & $\begin{array}{l}5 \\
5 \\
5 \\
4 \\
4 \\
4 \\
4 \\
3 \\
3\end{array}$ & $\begin{array}{l}5 \\
5 \\
5 \\
5 \\
5 \\
5 \\
5 \\
5 \\
5\end{array}$ & $\begin{array}{l}5 \\
5 \\
5 \\
5 \\
5 \\
5 \\
5 \\
5 \\
5\end{array}$ \\
\hline $\begin{array}{l}\text { Polyester } \\
\text { Fiberglass Board }\end{array}$ & $\begin{array}{r}0 \\
7 \\
14 \\
21 \\
28 \\
35 \\
42 \\
49 \\
56\end{array}$ & $\begin{array}{l}4 \\
4 \\
4 \\
4 \\
4 \\
4 \\
4 \\
4 \\
4\end{array}$ & $\begin{array}{l}5 \\
5 \\
5 \\
5 \\
5 \\
5 \\
5 \\
5 \\
4\end{array}$ & $\begin{array}{l}5 \\
5 \\
5 \\
5 \\
5 \\
4 \\
4 \\
4 \\
4\end{array}$ & $\begin{array}{l}5 \\
5 \\
5 \\
5 \\
5 \\
3 \\
3 \\
3 \\
3\end{array}$ & $\begin{array}{l}5 \\
5 \\
5 \\
5 \\
5 \\
5 \\
5 \\
5 \\
5\end{array}$ & $\begin{array}{l}5 \\
5 \\
5 \\
5 \\
5 \\
5 \\
5 \\
5 \\
5\end{array}$ \\
\hline $\begin{array}{l}\text { Lexan } \\
\text { Polycarbonate }\end{array}$ & $\begin{array}{r}0 \\
7 \\
14 \\
21 \\
28 \\
35 \\
42 \\
49 \\
56\end{array}$ & $\begin{array}{l}4 \\
4 \\
4 \\
4 \\
4 \\
4 \\
4 \\
4 \\
4\end{array}$ & $\begin{array}{c}5 \\
5 \\
5 \\
5 \\
5 \\
5 \\
5 \\
5 \\
4-5\end{array}$ & $\begin{array}{l}5 \\
5 \\
5 \\
4 \\
4 \\
4 \\
4 \\
4 \\
4\end{array}$ & $\begin{array}{l}5 \\
5 \\
5 \\
4 \\
4 \\
4 \\
4 \\
3 \\
3\end{array}$ & $\begin{array}{l}5 \\
5 \\
5 \\
5 \\
5 \\
5 \\
5 \\
5 \\
5\end{array}$ & $\begin{array}{l}5 \\
5 \\
5 \\
5 \\
5 \\
5 \\
5 \\
5 \\
5\end{array}$ \\
\hline $\begin{array}{l}\text { Mil. Spec. } \\
\text { Polyurethane } \\
\text { Painted Steel }\end{array}$ & $\begin{array}{r}0 \\
7 \\
14 \\
21 \\
28 \\
35 \\
42 \\
49 \\
56 \\
\end{array}$ & $\begin{array}{l}4 \\
4 \\
4 \\
4 \\
4 \\
4 \\
4 \\
4 \\
4 \\
\end{array}$ & $\begin{array}{c}5 \\
5 \\
5 \\
5 \\
5 \\
5 \\
5 \\
5 \\
4-5 \\
\end{array}$ & $\begin{array}{l}5 \\
5 \\
5 \\
5 \\
5 \\
5 \\
5 \\
5 \\
5 \\
\end{array}$ & $\begin{array}{l}5 \\
5 \\
5 \\
5 \\
5 \\
4 \\
4 \\
3 \\
3 \\
\end{array}$ & $\begin{array}{l}5 \\
5 \\
5 \\
5 \\
5 \\
5 \\
5 \\
5 \\
5 \\
\end{array}$ & $\begin{array}{l}5 \\
5 \\
5 \\
5 \\
5 \\
5 \\
5 \\
5 \\
5\end{array}$ \\
\hline
\end{tabular}

a Sce Table 3.8 for a description of the numerical ranking code for assessing tamper.tapes. 
Table 3.15. Results of Thermal Cycling Exposure $\left(-54^{\circ} \mathrm{C}\right.$ to $\left.46^{\circ} \mathrm{C}\right)$ for 2.54 -Centimeter-Wide Confirm ${ }^{\circledR}$ Window Tamper Tapes with Pressure Sensitive Adhesive and Vinyl Underlay (Number and Bar Code)

\begin{tabular}{|c|c|c|c|c|c|c|c|}
\hline \multirow[b]{3}{*}{ Surface } & \multirow[b]{3}{*}{$\begin{array}{c}\text { Days of } \\
\text { Exposure }\end{array}$} & \multicolumn{6}{|c|}{ Condition of Tamper Tape ${ }^{a}$} \\
\hline & & \multicolumn{2}{|c|}{ Appearance } & \multicolumn{2}{|c|}{ Security Emblem } & \multicolumn{2}{|c|}{ Adhesion to Surface } \\
\hline & & $\begin{array}{c}\text { Window } \\
\text { Area }\end{array}$ & $\begin{array}{l}\text { Vinyl } \\
\text { Area }\end{array}$ & $\begin{array}{c}\text { Window } \\
\text { Area }\end{array}$ & $\begin{array}{l}\text { Vinyl } \\
\text { Area }\end{array}$ & $\begin{array}{c}\text { Window } \\
\text { Area }\end{array}$ & Overall \\
\hline Steel & $\begin{array}{r}0 \\
7 \\
14 \\
21 \\
28 \\
35 \\
42 \\
49 \\
56\end{array}$ & $\begin{array}{l}4 \\
4 \\
4 \\
4 \\
4 \\
4 \\
4 \\
4 \\
4\end{array}$ & $\begin{array}{l}5 \\
5 \\
5 \\
5 \\
5 \\
5 \\
5 \\
5 \\
5\end{array}$ & $\begin{array}{l}5 \\
5 \\
5 \\
5 \\
5 \\
5 \\
5 \\
5 \\
5\end{array}$ & $\begin{array}{l}5 \\
5 \\
5 \\
5 \\
5 \\
5 \\
5 \\
5 \\
5\end{array}$ & $\begin{array}{l}5 \\
5 \\
5 \\
5 \\
5 \\
5 \\
5 \\
5 \\
5\end{array}$ & $\begin{array}{l}5 \\
5 \\
5 \\
5 \\
5 \\
5 \\
5 \\
5 \\
5\end{array}$ \\
\hline Aluminum & $\begin{array}{r}0 \\
7 \\
14 \\
21 \\
28 \\
35 \\
42 \\
49 \\
56\end{array}$ & $\begin{array}{l}4 \\
4 \\
4 \\
4 \\
4 \\
4 \\
4 \\
4 \\
4\end{array}$ & $\begin{array}{l}5 \\
5 \\
5 \\
5 \\
5 \\
5 \\
5 \\
5 \\
5\end{array}$ & $\begin{array}{l}5 \\
5 \\
5 \\
5 \\
5 \\
5 \\
5 \\
5 \\
5\end{array}$ & $\begin{array}{l}5 \\
5 \\
5 \\
5 \\
5 \\
5 \\
5 \\
5 \\
4\end{array}$ & $\begin{array}{l}5 \\
5 \\
5 \\
5 \\
5 \\
5 \\
5 \\
5 \\
5\end{array}$ & $\begin{array}{l}5 \\
5 \\
5 \\
5 \\
5 \\
5 \\
5 \\
5 \\
5\end{array}$ \\
\hline Wood & $\begin{array}{r}0 \\
7 \\
14 \\
21 \\
28 \\
35 \\
42 \\
49 \\
56\end{array}$ & $\begin{array}{l}5 \\
5 \\
5 \\
5 \\
5 \\
5 \\
5 \\
5 \\
5\end{array}$ & $\begin{array}{l}5 \\
5 \\
5 \\
5 \\
5 \\
5 \\
5 \\
5 \\
5\end{array}$ & $\begin{array}{l}5 \\
5 \\
5 \\
5 \\
5 \\
5 \\
5 \\
5 \\
5\end{array}$ & $\begin{array}{l}5 \\
5 \\
5 \\
5 \\
5 \\
5 \\
5 \\
5 \\
5\end{array}$ & $\begin{array}{l}5 \\
5 \\
5 \\
5 \\
5 \\
5 \\
5 \\
5 \\
5\end{array}$ & $\begin{array}{l}5 \\
5 \\
5 \\
5 \\
5 \\
5 \\
5 \\
5 \\
5\end{array}$ \\
\hline $\begin{array}{l}\text { Polyester } \\
\text { Fiberglass } \\
\text { Board }\end{array}$ & $\begin{array}{r}0 \\
7 \\
14 \\
21 \\
28 \\
35 \\
42 \\
49 \\
56\end{array}$ & $\begin{array}{l}4 \\
4 \\
4 \\
4 \\
4 \\
4 \\
4 \\
4 \\
4\end{array}$ & $\begin{array}{l}5 \\
5 \\
5 \\
5 \\
5 \\
5 \\
5 \\
5 \\
5\end{array}$ & $\begin{array}{l}5 \\
5 \\
5 \\
5 \\
5 \\
5 \\
5 \\
5 \\
5\end{array}$ & $\begin{array}{l}5 \\
5 \\
5 \\
5 \\
5 \\
5 \\
5 \\
5 \\
5\end{array}$ & $\begin{array}{l}5 \\
5 \\
5 \\
5 \\
5 \\
5 \\
5 \\
5 \\
5\end{array}$ & $\begin{array}{l}5 \\
5 \\
5 \\
5 \\
5 \\
5 \\
5 \\
5 \\
5\end{array}$ \\
\hline
\end{tabular}

a See Table 3.8 for a description of the numerical ranking code for assessing tamper tapes. 
Table 3.15. (Continued)

\begin{tabular}{|c|c|c|c|c|c|c|c|}
\hline \multirow[b]{3}{*}{ Surface } & \multirow[b]{3}{*}{$\begin{array}{c}\text { Days of } \\
\text { Exposurc }\end{array}$} & \multicolumn{6}{|c|}{ Condition of Tàmper Tape ${ }^{a}$} \\
\hline & & \multicolumn{2}{|c|}{ Appearance } & \multicolumn{2}{|c|}{ Security Emblem } & \multicolumn{2}{|c|}{ Adhesion to Surface } \\
\hline & & $\begin{array}{c}\text { Window } \\
\text { Area }\end{array}$ & $\begin{array}{l}\text { Vinyl } \\
\text { Area }\end{array}$ & $\begin{array}{c}\text { Window } \\
\text { Area } \\
\end{array}$ & $\begin{array}{l}\text { Vinyl } \\
\text { Area }\end{array}$ & $\begin{array}{c}\text { Window } \\
\text { Area }\end{array}$ & Overall \\
\hline $\begin{array}{l}\text { Lexan } \\
\text { Polycarbonate }\end{array}$ & $\begin{array}{r}0 \\
7 \\
14 \\
21 \\
28 \\
35 \\
42 \\
49 \\
56\end{array}$ & $\begin{array}{l}4 \\
4 \\
4 \\
4 \\
4 \\
4 \\
4 \\
4 \\
4\end{array}$ & $\begin{array}{l}5 \\
5 \\
5 \\
5 \\
5 \\
5 \\
5 \\
5 \\
5\end{array}$ & $\begin{array}{l}5 \\
5 \\
5 \\
5 \\
5 \\
5 \\
5 \\
5 \\
5\end{array}$ & $\begin{array}{l}5 \\
5 \\
5 \\
5 \\
5 \\
5 \\
5 \\
5 \\
5\end{array}$ & $\begin{array}{l}5 \\
5 \\
5 \\
5 \\
5 \\
5 \\
5 \\
5 \\
5\end{array}$ & $\begin{array}{l}5 \\
5 \\
5 \\
5 \\
5 \\
5 \\
5 \\
5 \\
5\end{array}$ \\
\hline $\begin{array}{l}\text { Mil. Spec. } \\
\text { Polyurethane } \\
\text { Painted Steel }\end{array}$ & $\begin{array}{c}0 \\
7 \\
14 \\
21 \\
28 \\
35 \\
42 \\
49 \\
56\end{array}$ & $\begin{array}{l}4 \\
4 \\
4 \\
4 \\
4 \\
4 \\
4 \\
4 \\
4\end{array}$ & $\begin{array}{l}5 \\
5 \\
5 \\
5 \\
5 \\
5 \\
5 \\
5 \\
5\end{array}$ & $\begin{array}{l}5 \\
5 \\
5 \\
5 \\
5 \\
5 \\
5 \\
5 \\
5\end{array}$ & $\begin{array}{l}5 \\
5 \\
5 \\
5 \\
5 \\
5 \\
5 \\
5 \\
5\end{array}$ & $\begin{array}{l}5 \\
5 \\
5 \\
5 \\
5 \\
5 \\
5 \\
5 \\
5\end{array}$ & $\begin{array}{l}5 \\
5 \\
5 \\
5 \\
5 \\
5 \\
5 \\
5 \\
5\end{array}$ \\
\hline
\end{tabular}

a See Table 3.8 for a description of the numerical ranking code for assessing tamper tapes.

\subsubsection{Transfer Resistance}

Three additional sets of the 12/93 PSA prototype tamper tape were bonded to four surfaces (roughened aluminum, roughened steel, fiberglass board, and polyurethane-painted steel) after which their transfer resistance was evaluated after 14 days. One set was the control kept at $23^{\circ} \mathrm{C}$ and 50\% relative humidity, the second set was kept in the QUV cabinet, and the third set was exposed in the thermal cycling cabinet. Another set of two 12/93 tamper tapes were each allowed to set for 30 minutes at room temperature before trying to remove them from the four surfaces. This test was designed to evaluate the transfer resistance of the 12/93 prototype tamper tapes and determine whether the adhesion of the PSA improves with time.

The data on the removal of the 12/93 tamper tapes is given in Table 3.16. After.30 minutes, the tamper tapes could be removed from all four of the surfaces, with damage to the Confirm ${ }^{\circledR}$ occurring only on the smooth fiberglass board surface. Comparing the adhesion of the tamper tapes at room temperature 30 minutes after application to those aged for 14 days shows that time improved adhesion on the polyurethane-painted surface, i.e., after 14 days adhesive transfer occurred or residue was left on the surface. No change was observed on the other smooth surface 
Table 3.16. Summary of Transfer Resistance of 12/93 Prototype Tamper Tapes

\begin{tabular}{|c|c|c|c|c|}
\hline Surface & $\begin{array}{c}30 \text { minutes } \\
23^{\circ} \mathrm{C}\end{array}$ & $\begin{array}{l}14 \text { days } \\
\text { Control }^{\mathrm{a}} \\
\end{array}$ & $\begin{array}{c}14 \text { days } \\
\text { QUV Cabinet }\end{array}$ & $\begin{array}{c}14 \text { days } \\
\text { Thermal Cycling } \\
\text { Cabinetc } \\
\end{array}$ \\
\hline $\begin{array}{l}\text { Roughed } \\
\text { Aluminum }\end{array}$ & $\begin{array}{l}\text { Both tamper tapes } \\
\text { removed, no } \\
\text { damage }\end{array}$ & $\begin{array}{l}\text { Tamper tape } \\
\text { removed, no } \\
\text { damage }\end{array}$ & $\begin{array}{l}\text { Tamper tape } \\
\text { removed, slight } \\
\text { damage to } \\
\text { Confirm }{ }^{\circledR}\end{array}$ & $\begin{array}{l}\text { Tamper tape } \\
\text { removed, no } \\
\text { damage }\end{array}$ \\
\hline $\begin{array}{l}\text { Roughened } \\
\text { Steel }\end{array}$ & $\begin{array}{l}\text { Both tamper tapes } \\
\text { removed, no } \\
\text { damage }\end{array}$ & $\begin{array}{l}\text { Tamper tape } \\
\text { removed, adhesive } \\
\text { transfer and } \\
\text { adhesive residue } \\
\text { on surface }\end{array}$ & $\begin{array}{l}\text { Tamper tape } \\
\text { removed, slight } \\
\text { damage to } \\
\text { Confirm }{ }^{\circledR} \text {, } \\
\text { adhesive transfer } \\
\text { and residue on } \\
\text { surface }\end{array}$ & $\begin{array}{l}\text { Tamper tape } \\
\text { removed, } \\
\text { Confirm }{ }^{\circledR} \\
\text { damaged, adhesive } \\
\text { transfer and } \\
\text { residue on surface }\end{array}$ \\
\hline $\begin{array}{l}\text { Polyester } \\
\text { Fiberglass } \\
\text { Board }\end{array}$ & $\begin{array}{l}\text { Both tamper tapes } \\
\text { removed, slight } \\
\text { damage to } \\
\text { Confirm }{ }^{\circledR}\end{array}$ & $\begin{array}{l}\text { Tamper tape } \\
\text { removed, slight } \\
\text { damage to } \\
\text { Confirm }{ }^{\circledR}\end{array}$ & $\begin{array}{l}\text { Tamper tape } \\
\text { removed, slight } \\
\text { damage to } \\
\text { Confirm }{ }^{\circledR}\end{array}$ & $\begin{array}{l}\text { Tamper tape } \\
\text { removed, some } \\
\text { damage to } \\
\text { Confirm }^{\circledR}\end{array}$ \\
\hline $\begin{array}{l}\text { Polyurethane } \\
\text { Painted Steel }\end{array}$ & $\begin{array}{l}\text { Both tamper tapes } \\
\text { removed, no } \\
\text { damage }\end{array}$ & $\begin{array}{l}\text { Tamper tape } \\
\text { removed, adhesive } \\
\text { residue on paint } \\
\text { surface, slight } \\
\text { damage to } \\
\text { Confirm }{ }^{\circledR}\end{array}$ & $\begin{array}{l}\text { Tamper tape } \\
\text { removed, adhesive } \\
\text { transfer occurred }\end{array}$ & $\begin{array}{l}\text { Tamper tape } \\
\text { removed, slight } \\
\text { damage to } \\
\text { Confirm }{ }^{\circledR} \text {, } \\
\text { adhesive transfer } \\
\text { and residue on } \\
\text { surface }\end{array}$ \\
\hline
\end{tabular}

a $23^{\circ} \mathrm{C}, 50 \%$ relative humidity

${ }^{b}$ Cycling of UV light at $60^{\circ} \mathrm{C}$ with condensing humidity at $40^{\circ} \mathrm{C}$

c Cycling between $-18^{\circ} \mathrm{C}$ to $46^{\circ} \mathrm{C}$

(fiberglass board), i.e., the tamper tapes were removed after aging with no adhesive or residue left on the surface. Improved adhesion with age also occurred on the roughened steel surface, as adhesive transfer and adhesive residue were found after removal of the aged tamper tape.

However, no change was observed after aging on the other roughened surface (aluminum). These results indicate that the adhesion of the PSA increases with time, improving the transfer resistance of the tamper tapes on some surfaces. However, the PSA is still inadequate as it allows transfer without indication of tampering on some surfaces.

\subsubsection{Abrasion Resistance}

A modification of ASTM Method D968, "Abrasion Resistance of Coatings of Paint, Varnish, Lacquer, and Related Products by the Falling Sand Method," was used to evaluate the abrasion 
resistance of the $12 / 93$ prototype tamper tapes. In this evaluation, flowing sand from a tube contacted the tamper tape at an angle of $75^{\circ}$ to $80^{\circ}$ such that the sand was in contact with the entire bar code area and over an entire security emblem of the Confirm ${ }^{\circledR}$. The purpose of the test was to determine the amount of sand needed to make the bar code unreadable by a bar code reader, or the amount of sand needed to destroy the security emblem as determined by a $3 \mathrm{M}$ Company security light.

A photograph of the sand abrasion apparatus is shown in Figure 3.1. Two kg of sand were placed in the funnel at the top of the apparatus for each pass. For each pass, the sand exited the bottom of the $0.9-\mathrm{m}$ sand dropping tube and landed on the tamper tape. Table 3.17 gives the

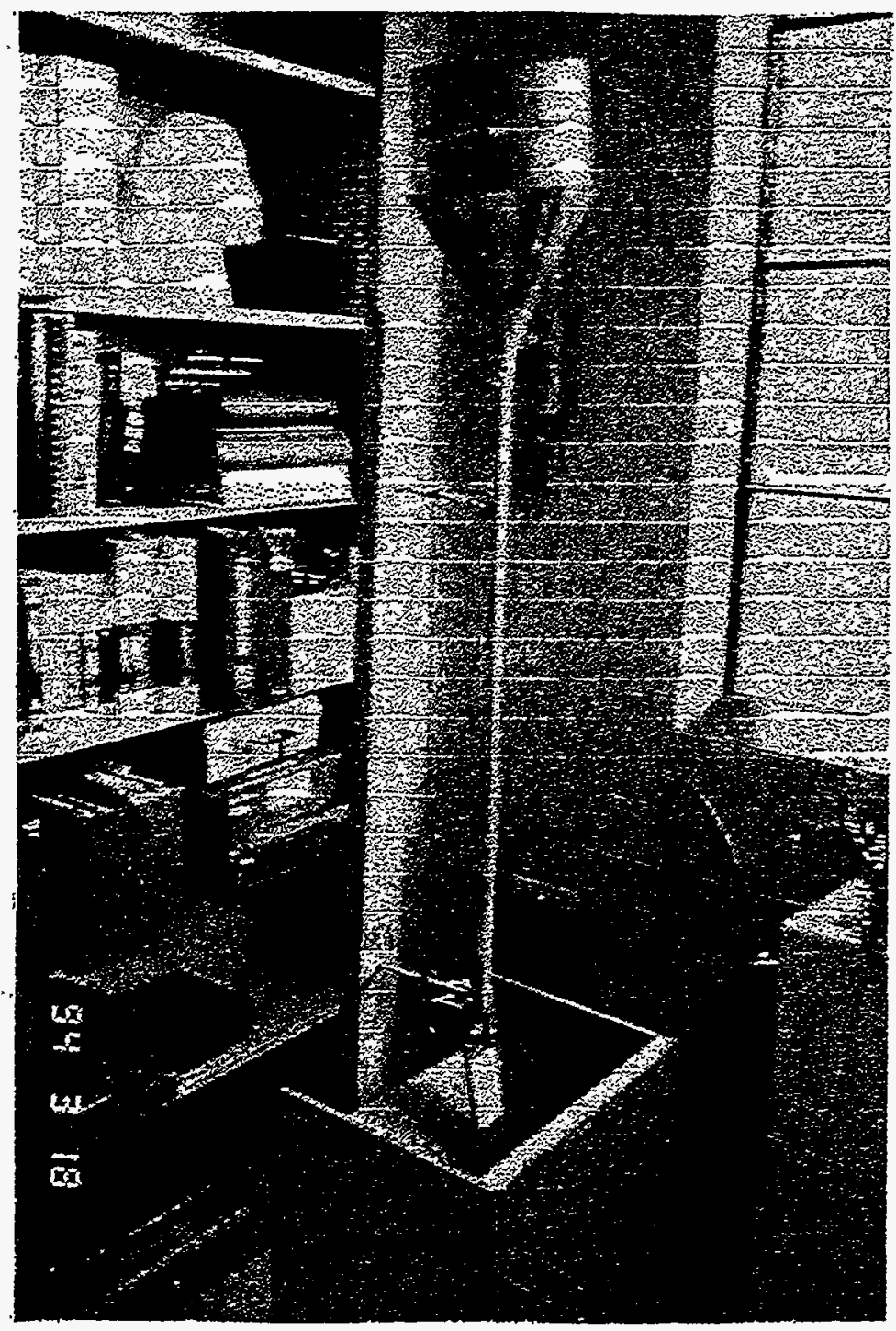

Figure 2.1. Sand Abrasion Apparatus 
Table 3.17. Abrasion Resistance of 12/93 Prototype Tamper Tape with Sand

\begin{tabular}{ccc}
\hline $\begin{array}{c}\text { Number of Passes } \\
(2 \mathrm{~kg} / \text { pass })\end{array}$ & \multicolumn{1}{c}{ Results } \\
\hline 3 & ${\text { Bar } \text { Code }^{\mathrm{a}}}^{\text {Some fading of bar code }}$ & Confirm $^{\circledR}$ Area $^{\mathrm{b}}$ \\
\hline 4 & Security emblem half gone \\
5 & More fading of bar code & \\
6 & More fading of bar code & Security emblem destroyed \\
\hline
\end{tabular}

a Using a bar code reader.

$\mathrm{b}$ Using 3M Company security light.

${ }^{c}$ More of the bar code was removed on the Confirm ${ }^{\circledR}$ than on the vinyl portion of the tamper tape.

results of the test. For the bar code area, there was indication that the ink faded more and more after two passes of sand. The ink was sufficiently removed after six passes of sand to prevent the bar code from being read with a reader. The abrasion resistance of the Confirm ${ }^{\circledR}$ was evaluated over one of the security emblems in the window area of the tamper tape. After three passes of sand, one-half of the emblem was gone as determined with the security light. The entire emblem was destroyed after five passes of sand. 


\subsection{TAMPER TAPES WITH RAPID-SET ADHESIVES}

One of the findings of the previous work with prototype tamper tapes with PSA was that the PSA did not sufficiently adhere to provide the level of tamper resistance desired. It was determined that the security performance of the tamper tapes could be increased by eliminating the PSA on the tamper tapes and substituting a more suitable reactive, rapid-set adhesive. Extensive adhesive formulation work was performed to obtain a rapid-set adhesive with the best balance of (1) fast cure time, (2) good adhesion to a wide range of test surfaces, and (3) resistance to any method of mechanical, heat, and solvent attack that an adversary could use to remove the tamper tape without damaging it. Formulation work was carried out using commercially-available resin and hardening components that included three types of reactive adhesives: polyurethanes, epoxies, and acrylics. The rapid-set adhesives were evaluated by bonding tamper tapes to a wide variety of surfaces. Commercially-available, two-component adhesives were also evaluated. As a result of these evaluations, four candidate rapid-set adhesives (two epoxies, one polyurethane, and one commercial acrylic) were chosen for further evaluation in weathering studies.

Two weathering studies with tamper tapes bonded to surfaces using the four candidate rapidset adhesives were performed. In the first study, tamper tapes with $2.54-\mathrm{cm}$-wide windows made of Confirm ${ }^{\circledR} 1700$ material were evaluated. In the second study, tamper tapes with $2.54-\mathrm{cm}$-wide windows made of three alternate Confirm ${ }^{\circledR}$ materials were evaluated.

\subsection{WEATHERING OF CONFIRM® 1700 TAMPER TAPES}

Tamper tapes with $2.54-\mathrm{cm}$-wide Confirm ${ }^{\circledR}$ windows were made in the laboratory out of Confirm ${ }^{\circledR} 1700$ and vinyl underlay materials bonded using a PSA supplied by $3 \mathrm{M}$ Company. The PSA was applied to the vinyl, the window area was cut out, and the vinyl was then bonded to the Confirm ${ }^{\circledR}$ material. PSA was used to bond the Confirm ${ }^{\circledR}$ and underlay.material since it was found in previous studies that poor weathering characteristics occurred when reactive, rapid-set adhesives were used to bond the vinyl to the Confirm ${ }^{\circledR}$ material. Using a rapid-set adhesive for a structural adhesive may have caused the poor weathering results for two reasons. First, the adhesives caused the Confirm ${ }^{\circledR}$ material to bond sufficiently to the release liner so that some of the Confirm ${ }^{\circledR}$ was separated in removing the release liner, thereby allowing weathering effects to accelerate degradation. Second, some of the adhesives, especially the acrylic type, attacked the Confirm ${ }^{\circledR}$ material. This "attack" allowed the entire area of the Confirm ${ }^{\circledR}$ material to degrade, not just the material in the window area. 
Each of four candidate reactive, rapid-set adhesives (epoxy 1, epoxy 2, polyurethane, and acrylic) were used to bond the prototype tamper tapes made in the laboratory to six surfaces, i.e., roughened steel, roughened aluminum, cedar wood, polyester fiberglass board, Lexan polycarbonate, and Mil. Spec. polyurethane-painted steel. Approximately $0.7 \mathrm{~g}$ of each of the candidate adhesives were applied manually to the tamper tapes using a small brush. The tamper tapes were then applied to the various test surfaces and were allowed to cure for several days. They were then tested in the QUV cabinet (ultraviolet light at $60^{\circ} \mathrm{C}$; condensing humidity at $40^{\circ} \mathrm{C}$ ), in the thermal cycling cabinet $\left(-18^{\circ} \mathrm{C}\right.$ to $\left.46^{\circ} \mathrm{C}\right)$, in a constant temperature room $\left(23^{\circ} \mathrm{C}, 50 \%\right.$ relative humidity) to serve as a control, and in Florida (a photograph of the Florida exposure site is provided in Figure 4.2). After weathering, the tamper tapes were visually examined by two or three persons to provide information regarding their appearance, the security feature with the $3 \mathrm{M}$ security illuminator, and the adhesion of the tamper tapes to the surfaces.

\subsubsection{Florida Exposure Results}

The results of the Daytona Beach, Florida exposure are given in Table 4.1. After 2 months of exposure, neither of the epoxy adhesive-bonded tamper tapes showed a loss of adhesion or changes in the appearance of the security feature. The security features in the window area of the tamper tapes bonded with the polyurethane adhesive or the acrylic adhesive were very faded or completely degraded. There was no loss of adhesion.

\subsubsection{QUV Cabinet Exposure Results}

The results for rapid-set adhesive tamper tapes bonded to various surfaces and exposed in the QUV cabinet for 56 days are given in Table 4.2. All of the candidate rapid-set adhesives had very good to excellent adhesion on all surfaces, except for the epoxy 1 adhesive on Lexan poly-

carbonate. The acrylic adhesive attacked the Confirm ${ }^{\circledR}$ (series 1700), solvating it so the window area wrinkled or "melted" badly. More importantly, it caused the security features to be faded and barely visible when examined with the $3 \mathrm{M}$ illuminator. The window area was black or grayishblack. Since the tamper tape was in bad shape at the beginning of the exposure, it only took 2 to 3 weeks in the QUV cabinet before the security features were compromised.

The Confirm ${ }^{\circledR}$ security features of the tamper tapes bonded with the polyurethane adhesive were very faded after 4 to 7 weeks of exposure. The performance of the tamper tapes bonded with epoxy 1 adhesive, relative to the rate of degradation, depended on the surface to which it was applied. On Lexan, the tamper tape started losing adhesion after 1 week, which led to quick degradation of the security features in 2 weeks. On fiberglass board, the security 


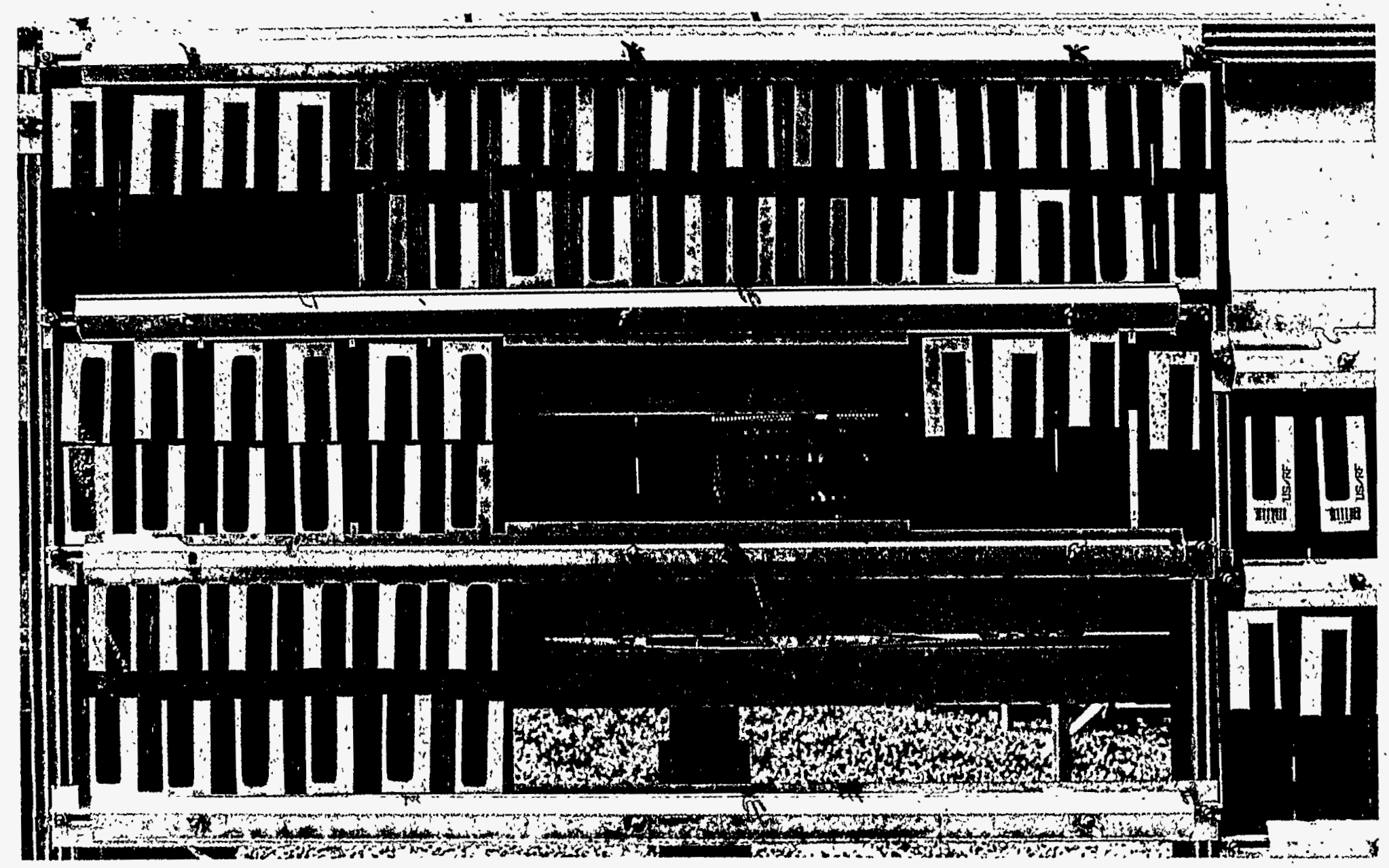

Figure 4.2. Photograph of Daytona Beach, Florida Exposure Site 
Table 4.1. Evaluation of Tamper Tapes Applied with Candidate Rapid-Set Adhesives and Aged in Daytona Beach, Florida at $90^{\circ}$ South

\begin{tabular}{|c|c|c|c|c|c|c|c|c|}
\hline \multirow[b]{3}{*}{ Surface } & \multirow[b]{3}{*}{ Adhesive } & \multirow{3}{*}{$\begin{array}{c}\text { Months } \\
\text { Exposure }\end{array}$} & \multicolumn{6}{|c|}{ Condition of Tamper Tape ${ }^{a}$} \\
\hline & & & \multicolumn{2}{|c|}{ Appearance } & \multicolumn{2}{|c|}{ Security Emblem } & \multicolumn{2}{|c|}{ Adhesion } \\
\hline & & & $\begin{array}{c}\text { Window } \\
\text { Area }\end{array}$ & $\begin{array}{l}\text { Vinyl } \\
\text { Area }\end{array}$ & $\begin{array}{c}\text { Window } \\
\text { Area }\end{array}$ & $\begin{array}{l}\text { Vinyl } \\
\text { Area }\end{array}$ & $\begin{array}{c}\text { Window } \\
\text { Area }\end{array}$ & Overall \\
\hline Aluminum & $\begin{array}{c}\text { epoxy } 1 \\
\text { epoxy } 2 \\
\text { polyurethane } \\
\text { acrylic }\end{array}$ & 0 & $\begin{array}{l}5 \\
5 \\
5 \\
5\end{array}$ & $\begin{array}{l}5 \\
5 \\
5 \\
5\end{array}$ & $\begin{array}{l}5 \\
5 \\
5 \\
5\end{array}$ & $\begin{array}{l}5 \\
5 \\
5 \\
5\end{array}$ & $\begin{array}{r}5 \\
5 \\
5 \\
5\end{array}$ & $\begin{array}{l}5 \\
5 \\
5 \\
5\end{array}$ \\
\hline Aluminum & $\begin{array}{c}\text { epoxy } 1 \\
\text { epoxy } 2 \\
\text { polyurethane } \\
\text { acrylic }\end{array}$ & 1 & $\begin{array}{c}4 \\
5 \\
4-5 \\
4-5\end{array}$ & $\begin{array}{l}4 \\
5 \\
5 \\
5\end{array}$ & $\begin{array}{l}5 \\
5 \\
5 \\
5\end{array}$ & $\begin{array}{l}5 \\
5 \\
5 \\
5\end{array}$ & $\begin{array}{l}5 \\
5 \\
5 \\
5\end{array}$ & $\begin{array}{l}5 \\
5 \\
5 \\
5\end{array}$ \\
\hline Aluminum & $\begin{array}{l}\text { epoxy } 1 \\
\text { epoxy } 2 \\
\text { polyurethane } \\
\text { acrylic }\end{array}$ & 2 & $\begin{array}{l}4 \\
5 \\
4 \\
4\end{array}$ & $\begin{array}{l}4 \\
5 \\
4 \\
4\end{array}$ & $\begin{array}{l}5 \\
5 \\
2 \\
2\end{array}$ & $\begin{array}{l}5 \\
5 \\
4 \\
5\end{array}$ & $\begin{array}{l}5 \\
5 \\
5 \\
5\end{array}$ & $\begin{array}{l}5 \\
5 \\
5 \\
5\end{array}$ \\
\hline Steel & $\begin{array}{c}\text { epoxy } 1 \\
\text { epoxy } 2 \\
\text { polyurethanc } \\
\text { acrylic }\end{array}$ & 0 & $\begin{array}{l}5 \\
5 \\
5 \\
5\end{array}$ & $\begin{array}{l}5 \\
5 \\
5 \\
5\end{array}$ & $\begin{array}{l}5 \\
5 \\
5 \\
5\end{array}$ & $\begin{array}{l}5 \\
5 \\
5 \\
5\end{array}$ & $\begin{array}{l}5 \\
5 \\
5 \\
5\end{array}$ & $\begin{array}{l}5 \\
5 \\
5 \\
5\end{array}$ \\
\hline Steel & $\begin{array}{c}\text { epoxy } 1 \\
\text { epoxy } 2 \\
\text { polyurethane } \\
\text { acrylic }\end{array}$ & 1 & $\begin{array}{c}4 \\
5 \\
4-5 \\
3-4\end{array}$ & $\begin{array}{c}4 \\
5 \\
5 \\
3-4\end{array}$ & $\begin{array}{l}5 \\
5 \\
5 \\
5\end{array}$ & $\begin{array}{l}5 \\
5 \\
5 \\
5\end{array}$ & $\begin{array}{l}5 \\
5 \\
5 \\
5\end{array}$ & $\begin{array}{l}5 \\
5 \\
5 \\
5\end{array}$ \\
\hline Steel & $\begin{array}{c}\text { epoxy } 1 \\
\text { epoxy } 2 \\
\text { polyurethane } \\
\text { acrylic }\end{array}$ & 2 & $\begin{array}{c}4 \\
5 \\
4 \\
4-5\end{array}$ & $\begin{array}{c}4 \\
5 \\
4 \\
4-5\end{array}$ & $\begin{array}{l}5 \\
5 \\
3 \\
1\end{array}$ & $\begin{array}{l}5 \\
5 \\
5 \\
4\end{array}$ & $\begin{array}{l}5 \\
5 \\
5 \\
5\end{array}$ & $\begin{array}{l}5 \\
5 \\
5 \\
5\end{array}$ \\
\hline Wood & $\begin{array}{c}\text { epoxy } 1 \\
\text { epoxy } 2 \\
\text { polyurethane } \\
\text { acrylic }\end{array}$ & 0 & $\begin{array}{c}5 \\
5 \\
5 \\
4-5\end{array}$ & $\begin{array}{c}5 \\
5 \\
5 \\
4-5\end{array}$ & $\begin{array}{l}5 \\
5 \\
5 \\
5\end{array}$ & $\begin{array}{l}5 \\
5 \\
5 \\
5\end{array}$ & $\begin{array}{l}5 \\
5 \\
5 \\
5\end{array}$ & $\begin{array}{l}5 \\
5 \\
5 \\
5\end{array}$ \\
\hline Wood & $\begin{array}{c}\text { epoxy } 1 \\
\text { epoxy } 2 \\
\text { polyurethane } \\
\text { acrylic }\end{array}$ & 1 & $\begin{array}{l}4-5 \\
5 \\
4-5 \\
3-4\end{array}$ & $\begin{array}{c}4-5 \\
5 \\
5 \\
3-4\end{array}$ & $\begin{array}{l}5 \\
5 \\
5 \\
5\end{array}$ & $\begin{array}{l}5 \\
5 \\
5 \\
5\end{array}$ & $\begin{array}{l}5 \\
5 \\
5 \\
5\end{array}$ & $\begin{array}{l}5 \\
5 \\
5 \\
5\end{array}$ \\
\hline Wood & $\begin{array}{c}\text { epoxy } 1 \\
\text { epoxy } 2 \\
\text { polyurethane } \\
\text { acrylic }\end{array}$ & 2 & $\begin{array}{c}4 \\
5 \\
4 \\
3-4\end{array}$ & $\begin{array}{c}4 \\
5 \\
4 \\
3-4\end{array}$ & $\begin{array}{l}5 \\
4 \\
3 \\
1\end{array}$ & $\begin{array}{l}5 \\
5 \\
4 \\
5\end{array}$ & $\begin{array}{l}5 \\
5 \\
5 \\
5\end{array}$ & $\begin{array}{l}5 \\
5 \\
5 \\
5 \\
\end{array}$ \\
\hline
\end{tabular}

a See Table 2.8 for a description of the numerical ranking code for assessing tamper tapes. 
Table 4.1. (Continued)

\begin{tabular}{|c|c|c|c|c|c|c|c|c|}
\hline \multirow[b]{3}{*}{ Surface } & \multirow[b]{3}{*}{ Adhesive } & \multirow[b]{3}{*}{$\begin{array}{c}\text { Months } \\
\text { Exposure }\end{array}$} & \multicolumn{6}{|c|}{ Condition of Tamper Tape ${ }^{a}$} \\
\hline & & & \multicolumn{2}{|c|}{ Appearance } & \multirow{2}{*}{$\begin{array}{c}\text { Security } \\
\text { Window } \\
\text { Area }\end{array}$} & \multirow{2}{*}{$\begin{array}{c}\text { Emblem } \\
\text { Vinyl } \\
\text { Area }\end{array}$} & \multicolumn{2}{|c|}{ Adhesion } \\
\hline & & & $\begin{array}{c}\text { Window } \\
\text { Area }\end{array}$ & $\begin{array}{l}\text { Vinyl } \\
\text { Area }\end{array}$ & & & $\begin{array}{c}\text { Window } \\
\text { Area }\end{array}$ & Overall \\
\hline $\begin{array}{l}\text { Fiberglass } \\
\text { Board }\end{array}$ & $\begin{array}{c}\text { epoxy } 1 \\
\text { epoxy } 2 \\
\text { polyurethane } \\
\text { acrylic }\end{array}$ & 0 & $\begin{array}{l}5 \\
5 \\
5 \\
5\end{array}$ & $\begin{array}{l}5 \\
5 \\
5 \\
5\end{array}$ & $\begin{array}{l}5 \\
5 \\
5 \\
5\end{array}$ & $\begin{array}{l}5 \\
5 \\
5 \\
5\end{array}$ & $\begin{array}{l}5 \\
5 \\
5 \\
5\end{array}$ & $\begin{array}{l}5 \\
5 \\
5 \\
5\end{array}$ \\
\hline $\begin{array}{l}\text { Fiberglass } \\
\text { Board }\end{array}$ & $\begin{array}{c}\text { epoxy } 1 \\
\text { epoxy } 2 \\
\text { polyurethane } \\
\text { acrylic }\end{array}$ & 1 & $\begin{array}{c}4 \\
5 \\
4-5 \\
5\end{array}$ & $\begin{array}{l}4 \\
5 \\
5 \\
5\end{array}$ & $\begin{array}{l}5 \\
5 \\
5 \\
5\end{array}$ & $\begin{array}{l}5 \\
5 \\
5 \\
5\end{array}$ & $\begin{array}{l}5 \\
5 \\
5 \\
5\end{array}$ & $\begin{array}{l}5 \\
5 \\
5 \\
5\end{array}$ \\
\hline $\begin{array}{l}\text { Fiberglass } \\
\text { Board }\end{array}$ & $\begin{array}{c}\text { epoxy } 1 \\
\text { epoxy } 2 \\
\text { polyurethane } \\
\text { acrylic }\end{array}$ & 2 & $\begin{array}{l}4 \\
5 \\
4 \\
5\end{array}$ & $\begin{array}{l}4 \\
5 \\
4 \\
5\end{array}$ & $\begin{array}{l}5 \\
5 \\
2 \\
2\end{array}$ & $\begin{array}{l}5 \\
5 \\
5 \\
5\end{array}$ & $\begin{array}{l}5 \\
5 \\
5 \\
5\end{array}$ & $\begin{array}{l}5 \\
5 \\
5 \\
5\end{array}$ \\
\hline Lexan & $\begin{array}{c}\text { epoxy } 1 \\
\text { epoxy } 2 \\
\text { polyurethane } \\
\text { acrylic }\end{array}$ & 0 & $\begin{array}{l}5 \\
5 \\
5 \\
5\end{array}$ & $\begin{array}{l}5 \\
5 \\
5 \\
5\end{array}$ & $\begin{array}{l}5 \\
5 \\
5 \\
5\end{array}$ & $\begin{array}{l}5 \\
5 \\
5 \\
5\end{array}$ & $\begin{array}{l}5 \\
5 \\
5 \\
5\end{array}$ & $\begin{array}{l}5 \\
5 \\
5 \\
5\end{array}$ \\
\hline Lexan & $\begin{array}{c}\text { epoxy } 1 \\
\text { epoxy } 2 \\
\text { polyurethane } \\
\text { acrylic }\end{array}$ & 1 & $\begin{array}{c}4 \\
5 \\
4-5 \\
4-5\end{array}$ & $\begin{array}{l}4 \\
5 \\
5 \\
5\end{array}$ & $\begin{array}{l}5 \\
5 \\
5 \\
5\end{array}$ & $\begin{array}{l}5 \\
5 \\
5 \\
5\end{array}$ & $\begin{array}{l}5 \\
5 \\
5 \\
5\end{array}$ & $\begin{array}{l}5 \\
5 \\
5 \\
5\end{array}$ \\
\hline Lexan & $\begin{array}{l}\text { epoxy } 1 \\
\text { epoxy } 2 \\
\text { polyurethane } \\
\text { acrylic }\end{array}$ & 2 & $\begin{array}{c}4 \\
5 \\
4 \\
4-5\end{array}$ & $\begin{array}{c}4 \\
5 \\
4 \\
4-5\end{array}$ & $\begin{array}{l}5 \\
5 \\
1 \\
1\end{array}$ & $\begin{array}{l}5 \\
5 \\
5 \\
4\end{array}$ & $\begin{array}{l}5 \\
5 \\
5 \\
5\end{array}$ & $\begin{array}{l}5 \\
5 \\
5 \\
5\end{array}$ \\
\hline $\begin{array}{l}\text { Mil. Spec. } \\
\text { PUb } \\
\text { Painted } \\
\text { Steel }\end{array}$ & $\begin{array}{l}\text { epoxy } 1 \\
\text { epoxy } 2 \\
\text { polyurethane } \\
\text { acrylic }\end{array}$ & 0 & $\begin{array}{c}5 \\
5 \\
5 \\
4-5\end{array}$ & $\begin{array}{c}5 \\
5 \\
5 \\
4-5\end{array}$ & $\begin{array}{l}5 \\
5 \\
5 \\
5\end{array}$ & $\begin{array}{l}5 \\
5 \\
5 \\
5\end{array}$ & $\begin{array}{l}5 \\
5 \\
5 \\
5\end{array}$ & $\begin{array}{l}5 \\
5 \\
5 \\
5\end{array}$ \\
\hline $\begin{array}{l}\text { Mil. Spec. } \\
\text { PU-Painted } \\
\text { Steel } \\
\end{array}$ & $\begin{array}{c}\text { epoxy } 1 \\
\text { epoxy } 2 \\
\text { polyurethane } \\
\text { acrylic }\end{array}$ & 1 & $\begin{array}{c}4 \\
5 \\
4-5 \\
3-4\end{array}$ & $\begin{array}{c}4 \\
5 \\
5 \\
3-4\end{array}$ & $\begin{array}{l}5 \\
5 \\
5 \\
5\end{array}$ & $\begin{array}{l}5 \\
5 \\
5 \\
5\end{array}$ & $\begin{array}{l}5 \\
5 \\
5 \\
5\end{array}$ & $\begin{array}{l}5 \\
5 \\
5 \\
5\end{array}$ \\
\hline $\begin{array}{l}\text { Mil. Spec. } \\
\text { PU-Painted } \\
\text { Steel }\end{array}$ & $\begin{array}{c}\text { cpoxy } 1 \\
\text { epoxy } 2 \\
\text { polyurethane } \\
\text { acrylic }\end{array}$ & 2 & $\begin{array}{c}4 \\
5 \\
4 \\
3-4 \\
\end{array}$ & $\begin{array}{c}4 \\
5 \\
4 \\
3-4\end{array}$ & $\begin{array}{l}5 \\
5 \\
1 \\
2\end{array}$ & $\begin{array}{l}5 \\
5 \\
4 \\
4\end{array}$ & $\begin{array}{l}5 \\
5 \\
5 \\
5\end{array}$ & $\begin{array}{l}5 \\
5 \\
5 \\
5\end{array}$ \\
\hline
\end{tabular}

a See Table 3.8 for a description of the numerical ranking code for assessing tamper tapes.

b Polyurethane 
Table 4.2. Evaluation of Tamper Tapes Applied with Candidate Rapid-Set Adhesives and Aged in QUV Cabinet (UV, $60^{\circ} \mathrm{C}$; Condensing Humidity, $40^{\circ} \mathrm{C}$ )

\begin{tabular}{|c|c|c|c|c|c|c|c|c|}
\hline \multirow[b]{3}{*}{ Surface } & \multirow[b]{3}{*}{ Adhesive } & \multirow[b]{3}{*}{$\begin{array}{l}\text { Days of } \\
\text { Exposure }\end{array}$} & \multicolumn{6}{|c|}{ Condition of Tamper Tape ${ }^{a}$} \\
\hline & & & \multicolumn{2}{|c|}{ Appearance } & \multicolumn{2}{|c|}{ Security Emblem } & \multicolumn{2}{|c|}{ Adhesion } \\
\hline & & & $\begin{array}{c}\text { Window } \\
\text { Area }\end{array}$ & $\begin{array}{l}\text { Vinyl } \\
\text { Area }\end{array}$ & $\begin{array}{c}\text { Window } \\
\text { Area }\end{array}$ & $\begin{array}{l}\text { Vinyl } \\
\text { Area }\end{array}$ & $\begin{array}{c}\text { Window } \\
\text { Area }\end{array}$ & Overall \\
\hline $\begin{array}{c}\text { Aluminum } \\
\text {. }\end{array}$ & epoxy 1 & $\begin{array}{r}0 \\
7 \\
14 \\
21 \\
28 \\
35 \\
42 \\
49 \\
56\end{array}$ & $\begin{array}{l}5 \\
4 \\
3 \\
3 \\
3 \\
3 \\
3 \\
3 \\
2\end{array}$ & $\begin{array}{l}5 \\
4 \\
3 \\
3 \\
3 \\
3 \\
3 \\
3 \\
2\end{array}$ & $\begin{array}{l}5 \\
5 \\
5 \\
4 \\
4 \\
3 \\
3 \\
3 \\
3\end{array}$ & $\begin{array}{l}5 \\
5 \\
5 \\
4 \\
4 \\
3 \\
3 \\
3 \\
3\end{array}$ & $\begin{array}{l}5 \\
5 \\
5 \\
5 \\
5 \\
5 \\
5 \\
5 \\
5\end{array}$ & $\begin{array}{l}5 \\
5 \\
5 \\
5 \\
5 \\
5 \\
5 \\
5 \\
5\end{array}$ \\
\hline Aluminum & epoxy 2 & $\begin{array}{r}0 \\
7 \\
14 \\
21 \\
28 \\
35 \\
42 \\
49 \\
56\end{array}$ & $\begin{array}{l}5 \\
5 \\
4 \\
4 \\
4 \\
4 \\
4 \\
4 \\
4\end{array}$ & $\begin{array}{l}5 \\
5 \\
4 \\
4 \\
4 \\
4 \\
4 \\
4 \\
4\end{array}$ & $\begin{array}{r}5 \\
5 \\
5 \\
5 \\
5 \\
5 \\
5 \\
5 \\
5 \\
5\end{array}$ & $\begin{array}{l}5 \\
5 \\
5 \\
5 \\
4 \\
4 \\
4 \\
4 \\
3\end{array}$ & $\begin{array}{r}5 \\
5 \\
5 \\
5 \\
5 \\
5 \\
5 \\
5 \\
5\end{array}$ & $\begin{array}{l}5 \\
5 \\
5 \\
5 \\
5 \\
5 \\
5 \\
5 \\
5\end{array}$ \\
\hline Aluminum & polyurethane & $\begin{array}{r}0 \\
7 \\
21 \\
28 \\
35 \\
42 \\
49\end{array}$ & $\begin{array}{l}5 \\
5 \\
5 \\
4 \\
3 \\
2 \\
. \mathrm{b}\end{array}$ & $\begin{array}{l}5 \\
5 \\
5 \\
5 \\
4 \\
4\end{array}$ & $\begin{array}{c}5 \\
5 \\
5 \\
4-5 \\
2 \\
1-2 \\
-\end{array}$ & $\begin{array}{l}5 \\
5 \\
5 \\
5 \\
4 \\
4\end{array}$ & $\begin{array}{l}5 \\
5 \\
5 \\
5 \\
5 \\
5 \\
-\end{array}$ & $\begin{array}{l}5 \\
5 \\
5 \\
5 \\
5 \\
5\end{array}$ \\
\hline Aluminum & acrylic & $\begin{array}{r}0 \\
7 \\
14 \\
21 \\
28 \\
35\end{array}$ & $\begin{array}{l}3 \\
3 \\
3 \\
3 \\
3 \\
-\end{array}$ & $\begin{array}{l}4 \\
4 \\
4 \\
4 \\
4\end{array}$ & $\begin{array}{l}3 \\
3 \\
1 \\
1 \\
1 \\
-\end{array}$ & $\begin{array}{l}3 \\
3 \\
3 \\
3 \\
3\end{array}$ & $\begin{array}{l}5 \\
5 \\
5 \\
5 \\
5 \\
-\end{array}$ & $\begin{array}{l}5 \\
5 \\
5 \\
5 \\
5\end{array}$ \\
\hline Steel & epoxy 1 & $\begin{array}{r}0 \\
7 \\
14 \\
21 \\
28 \\
35 \\
42 \\
49 \\
56\end{array}$ & $\begin{array}{l}5 \\
4 \\
4 \\
4 \\
4 \\
4 \\
4 \\
4 . \\
3\end{array}$ & $\begin{array}{l}5 \\
4 \\
4 \\
4 \\
4 \\
4 \\
4 \\
3 \\
3\end{array}$ & $\begin{array}{l}5 \\
5 \\
5 \\
4 \\
3 \\
3 \\
3 \\
3 \\
3\end{array}$ & $\begin{array}{l}5 \\
5 \\
5 \\
4 \\
4 \\
4 \\
4 \\
3 \\
2\end{array}$ & $\begin{array}{l}5 \\
5 \\
5 \\
5 \\
5 \\
5 \\
5 \\
5 \\
5\end{array}$ & $\begin{array}{l}5 \\
5 \\
5 \\
5 \\
5 \\
5 \\
5 \\
5 \\
5\end{array}$ \\
\hline
\end{tabular}

a See Table 3.8 for a description of the numerical ranking code for assessing tamper tapes.

b Samples were removed from QUV cabinet because their security features were compromised. 
Table 4.2. (Continued)

\begin{tabular}{|c|c|c|c|c|c|c|c|c|}
\hline \multirow[b]{3}{*}{ Surface } & \multirow[b]{3}{*}{ Adhesive } & \multirow[b]{3}{*}{$\begin{array}{c}\text { Days of } \\
\text { Exposure }\end{array}$} & \multicolumn{6}{|c|}{ Condition of Tamper Tape ${ }^{a}$} \\
\hline & & & \multicolumn{2}{|c|}{ Appearance } & \multirow{2}{*}{$\begin{array}{c}\text { Security } \\
\text { Window } \\
\text { Area }\end{array}$} & \multirow{2}{*}{$\begin{array}{c}\text { Emblem } \\
\text { Vinyl } \\
\text { Area }\end{array}$} & \multicolumn{2}{|c|}{ Adhesion } \\
\hline & & & $\begin{array}{c}\text { Window } \\
\text { Area }\end{array}$ & $\begin{array}{l}\text { Vinyl } \\
\text { Area } \\
\end{array}$ & & & $\begin{array}{c}\text { Window } \\
\text { Area }\end{array}$ & Overall \\
\hline Steel & epoxy 2 & $\begin{array}{r}0 \\
7 \\
14 \\
21 \\
28 \\
35 \\
42 \\
49 \\
56\end{array}$ & $\begin{array}{l}5 \\
5 \\
5 \\
5 \\
5 \\
5 \\
5 \\
5 \\
4\end{array}$ & $\begin{array}{l}5 \\
5 \\
5 \\
5 \\
5 \\
5 \\
5 \\
5 \\
3\end{array}$ & $\begin{array}{l}5 \\
5 \\
5 \\
5 \\
5 \\
5 \\
5 \\
5 \\
5\end{array}$ & $\begin{array}{l}5 \\
5 \\
5 \\
5 \\
4 \\
4 \\
4 \\
4 \\
3\end{array}$ & $\begin{array}{l}5 \\
5 \\
5 \\
5 \\
5 \\
5 \\
5 \\
5 \\
5\end{array}$ & $\begin{array}{l}5 \\
5 \\
5 \\
5 \\
5 \\
5 \\
5 \\
5 \\
5\end{array}$ \\
\hline Steel & polyurethane & $\begin{array}{r}0 \\
7 \\
14 \\
-\quad 21 \\
28 \\
35 \\
42 \\
49 \\
56\end{array}$ & $\begin{array}{l}4 \\
4 \\
4 \\
3 \\
3 \\
3 \\
3 \\
3 \\
2\end{array}$ & $\begin{array}{l}5 \\
5 \\
5 \\
4 \\
4 \\
4 \\
4 \\
4 \\
4\end{array}$ & $\begin{array}{l}5 \\
5 \\
5 \\
3 \\
1 \\
1 \\
1 \\
1 \\
1\end{array}$ & $\begin{array}{l}5 \\
5 \\
5 \\
4 \\
3 \\
3 \\
3 \\
3 \\
3\end{array}$ & $\begin{array}{l}5 \\
5 \\
5 \\
5 \\
5 \\
5 \\
5 \\
5 \\
5\end{array}$ & $\begin{array}{l}5 \\
5 \\
5 \\
5 \\
5 \\
5 \\
5 \\
5 \\
5\end{array}$ \\
\hline Steel & acrylic & $\begin{array}{r}0 \\
7 \\
14 \\
21 \\
28 \\
35\end{array}$ & $\begin{array}{l}3 \\
2 \\
2 \\
2 \\
2 \\
. \mathrm{b}\end{array}$ & $\begin{array}{l}4 \\
4 \\
4 \\
4 \\
4\end{array}$ & $\begin{array}{l}3 \\
1 \\
1 \\
1 \\
1 \\
-\end{array}$ & $\begin{array}{l}3 \\
2 \\
2 \\
2 \\
2\end{array}$ & $\begin{array}{l}5 \\
5 \\
5 \\
5 \\
5 \\
-\end{array}$ & $\begin{array}{l}5 \\
5 \\
5 \\
5 \\
5\end{array}$ \\
\hline Wood & epoxy 1 & $\begin{array}{r}0 \\
7 \\
14 \\
21 \\
28 \\
35 \\
42 \\
49 \\
56\end{array}$ & $\begin{array}{c}5 \\
4 \\
4 \\
3-4 \\
3 \\
3 \\
3 \\
3 \\
2\end{array}$ & $\begin{array}{c}5 \\
4 \\
4 \\
3-4 \\
3-4 \\
3-4 \\
3-4 \\
3 \\
2\end{array}$ & $\begin{array}{l}5 \\
5 \\
5 \\
5 \\
3 \\
3 \\
3 \\
3 \\
2\end{array}$ & $\begin{array}{l}5 \\
5 \\
5 \\
5 \\
4 \\
4 \\
4 \\
4 \\
4\end{array}$ & $\begin{array}{l}5 \\
5 \\
5 \\
5 \\
5 \\
5 \\
5 \\
5 \\
5\end{array}$ & $\begin{array}{l}5 \\
5 \\
5 \\
5 \\
5 \\
5 \\
5 \\
5 \\
5\end{array}$ \\
\hline Wood & epoxy 2 & $\begin{array}{r}0 \\
7 \\
14 \\
21 \\
28 \\
35 \\
42 \\
49 \\
56\end{array}$ & $\begin{array}{l}5 \\
5 \\
5 \\
4 \\
4 \\
4 \\
4 \\
4 \\
4\end{array}$ & $\begin{array}{l}5 \\
5 \\
5 \\
4 \\
4 \\
4 \\
4 \\
4 \\
4\end{array}$ & $\begin{array}{l}\cdot 5 \\
5 \\
5 \\
5 \\
5 \\
5 \\
5 \\
5 \\
5\end{array}$ & $\begin{array}{l}5 \\
5 \\
5 \\
5 \\
3 \\
3 \\
3 \\
3 \\
.3\end{array}$ & $\begin{array}{l}5 \\
5 \\
5 \\
5 \\
5 \\
5 \\
5 \\
5 \\
5\end{array}$ & $\begin{array}{r}5 \\
5 \\
5 \\
5 \\
5 \\
5 \\
5 \\
.5 \\
5\end{array}$ \\
\hline
\end{tabular}

a See Table 3.8 for a description of the numerical ranking code for assessing tamper tapes.

b Samples were removed from QUV cabinet because their security features were compromised. 
Table 4.2. (Continued)

\begin{tabular}{|c|c|c|c|c|c|c|c|c|}
\hline \multirow[b]{3}{*}{ Surface } & \multirow[b]{3}{*}{ Adhesive } & \multirow[b]{3}{*}{$\begin{array}{c}\text { Days of } \\
\text { Exposure }\end{array}$} & \multicolumn{6}{|c|}{ Condition of Tamper Tape ${ }^{a}$} \\
\hline & & & \multicolumn{2}{|c|}{ Appearance } & \multicolumn{2}{|c|}{ Security Emblem } & \multicolumn{2}{|c|}{ Adhesion } \\
\hline & & & $\begin{array}{c}\text { Window } \\
\text { Area }\end{array}$ & $\begin{array}{l}\text { Vinyl } \\
\text { Area }\end{array}$ & $\begin{array}{c}\text { Window } \\
\text { Area }\end{array}$ & $\begin{array}{l}\text { Vinyl } \\
\text { Area }\end{array}$ & $\begin{array}{c}\text { Window } \\
\text { Area }\end{array}$ & Overall \\
\hline Wood & polyurethane & $\begin{array}{r}0 \\
7 \\
14 \\
21 \\
28 \\
35 \\
42 \\
49 \\
56\end{array}$ & $\begin{array}{l}5 \\
5 \\
4 \\
3 \\
3 \\
3 \\
3 \\
3 \\
2\end{array}$ & $\begin{array}{l}5 \\
5 \\
5 \\
3 \\
3 \\
3 \\
3 \\
3 \\
3\end{array}$ & $\begin{array}{c}5 \\
5 \\
5 \\
5 \\
3 \\
3 \\
3 \\
2 \\
1-2\end{array}$ & $\begin{array}{l}5 \\
5 \\
5 \\
5 \\
3 \\
3 \\
3 \\
3 \\
3\end{array}$ & $\begin{array}{l}5 \\
5 \\
5 \\
5 \\
5 \\
5 \\
5 \\
5 \\
5\end{array}$ & $\begin{array}{l}5 \\
5 \\
5 \\
5 \\
5 \\
5 \\
5 \\
5 \\
5\end{array}$ \\
\hline Wood & acrylic & $\begin{array}{r}0 \\
7 \\
14 \\
21 \\
28 \\
35\end{array}$ & $\begin{array}{c}3 \\
3 \\
3 \\
2-3 \\
2-3 \\
-b\end{array}$ & $\begin{array}{l}4 \\
4 \\
4 \\
3 \\
3\end{array}$ & $\begin{array}{l}3 \\
3 \\
2 \\
2 \\
1 \\
-\end{array}$ & $\begin{array}{l}3 \\
3 \\
3 \\
3 \\
3\end{array}$ & $\begin{array}{l}5 \\
5 \\
5 \\
5 \\
5 \\
-\end{array}$ & $\begin{array}{l}5 \\
5 \\
5 \\
5 \\
5\end{array}$ \\
\hline $\begin{array}{l}\text { Fiberglass } \\
\text { Board }\end{array}$ & epoxy 1 & $\begin{array}{r}0 \\
7 \\
14 \\
21 \\
28 \\
35 \\
42 \\
49 \\
56\end{array}$ & $\begin{array}{c}5 \\
4 \\
4 \\
3-4 \\
3 \\
3 \\
3 \\
3 \\
2\end{array}$ & $\begin{array}{c}5 \\
4 \\
4 \\
3-4 \\
3 \\
3 \\
3 \\
3 \\
2\end{array}$ & $\begin{array}{l}5 \\
5 \\
5 \\
5 \\
2 \\
2 \\
2 \\
2 \\
2\end{array}$ & $\begin{array}{l}5 \\
5 \\
5 \\
5 \\
2 \\
2 \\
2 \\
2 \\
2\end{array}$ & $\begin{array}{l}5 \\
5 \\
5 \\
5 \\
5 \\
5 \\
5 \\
5 \\
5\end{array}$ & $\begin{array}{l}5 \\
5 \\
5 \\
5 \\
5 \\
5 \\
5 \\
5 \\
5\end{array}$ \\
\hline $\begin{array}{l}\text { Fiberglass } \\
\text { Board }\end{array}$ & epoxy 2 & $\begin{array}{r}0 \\
7 \\
14 \\
21 \\
28 \\
35 \\
42 \\
49 \\
56\end{array}$ & $\begin{array}{l}5 \\
5 \\
5 \\
4 \\
4 \\
4 \\
4 \\
4 \\
4\end{array}$ & $\begin{array}{c}5 \\
5 \\
5 \\
4-5 \\
4-5 \\
4-5 \\
4-5 \\
4-5 \\
4\end{array}$ & $\begin{array}{l}5 \\
5 \\
5 \\
5 \\
5 \\
5 \\
5 \\
5 \\
5\end{array}$ & $\begin{array}{l}5 \\
5 \\
5 \\
5 \\
4 \\
4 \\
4 \\
4 \\
3\end{array}$ & $\begin{array}{l}5 \\
5 \\
5 \\
5 \\
5 \\
5 \\
5 \\
5 \\
5\end{array}$ & $\begin{array}{l}5 \\
5 \\
5 \\
5 \\
5 \\
5 \\
5 \\
5 \\
5\end{array}$ \\
\hline $\begin{array}{l}\text { Fiberglass } \\
\text { Board }\end{array}$ & polyurethane & $\begin{array}{r}0 \\
7 \\
14 \\
21 \\
28 \\
35\end{array}$ & $\begin{array}{l}5 \\
5 \\
5 \\
3 \\
3 \\
-\end{array}$ & $\begin{array}{l}5 \\
5 \\
5 \\
4 \\
4\end{array}$ & $\begin{array}{l}5 \\
5 \\
5 \\
3 \\
1 \\
-\end{array}$ & $\begin{array}{l}5 \\
5 \\
5 \\
5 \\
3\end{array}$ & $\begin{array}{l}5 \\
5 \\
5 \\
5 \\
5 \\
-\end{array}$ & $\begin{array}{l}5 \\
5 \\
5 \\
5 \\
5\end{array}$ \\
\hline
\end{tabular}

a See Table 3.8 for a description of the numerical ranking code for assessing tamper tapes.

b Samples were removed from QUV cabinet because their security features were compromised. 
Table 4.2. (Continued)

\begin{tabular}{|c|c|c|c|c|c|c|c|c|}
\hline \multirow[b]{3}{*}{ Surface } & \multirow[b]{3}{*}{ Adhesive } & \multirow[b]{3}{*}{$\begin{array}{c}\text { Days of } \\
\text { Exposure }\end{array}$} & \multicolumn{6}{|c|}{ Condition of Tamper Tape ${ }^{a}$} \\
\hline & & & \multicolumn{2}{|c|}{ Appearance } & \multicolumn{2}{|c|}{ Security Emblem } & \multicolumn{2}{|c|}{ Adhesion } \\
\hline & & & $\begin{array}{c}\text { Window } \\
\text { Area }\end{array}$ & $\begin{array}{l}\text { Vinyl } \\
\text { Area } \\
\end{array}$ & $\begin{array}{c}\text { Window } \\
\text { Area }\end{array}$ & $\begin{array}{l}\text { Vinyl } \\
\text { Area } \\
\end{array}$ & $\begin{array}{c}\text { Window } \\
\text { Area }\end{array}$ & Overall \\
\hline $\begin{array}{l}\text { Fiberglass } \\
\text { Board }\end{array}$ & acrylic & $\begin{array}{r}0 \\
7 \\
14 \\
21 \\
28 \\
35\end{array}$ & $\begin{array}{l}3 \\
3 \\
3 \\
3 \\
3 \\
-b\end{array}$ & $\begin{array}{l}4 \\
4 \\
4 \\
4 \\
4\end{array}$ & $\begin{array}{l}3 \\
2 \\
1 \\
1 \\
1 \\
-\end{array}$ & $\begin{array}{l}3 \\
3 \\
3 \\
3 \\
3\end{array}$ & $\begin{array}{l}5 \\
5 \\
5 \\
5 \\
5 \\
-\end{array}$ & $\begin{array}{l}5 \\
5 \\
5 \\
5 \\
5\end{array}$ \\
\hline Lexan & epoxy 1 & $\begin{array}{r}0 \\
7 \\
14 \\
21 \\
28 \\
35\end{array}$ & $\begin{array}{l}5 \\
2 \\
1 \\
1 \\
1 \\
-\end{array}$ & $\begin{array}{l}5 \\
3 \\
2 \\
1 \\
1\end{array}$ & $\begin{array}{l}5 \\
5 \\
1 \\
1 \\
1 \\
-\end{array}$ & $\begin{array}{l}5 \\
5 \\
3 \\
3 \\
3\end{array}$ & $\begin{array}{l}5 \\
1 \\
1 \\
1 \\
1 \\
-\end{array}$ & $\begin{array}{c}5 \\
2-3 \\
1-2 \\
1 \\
1\end{array}$ \\
\hline Lexan & epoxy 2 & $\begin{array}{r}0 \\
7 \\
14 \\
21 \\
28 \\
35 \\
42 \\
49 \\
56\end{array}$ & $\begin{array}{l}5 \\
5 \\
5 \\
4 \\
4 \\
4 \\
4 \\
4 \\
4\end{array}$ & $\begin{array}{l}5 \\
5 \\
5 \\
4 \\
4 \\
4 \\
4 \\
4 \\
3\end{array}$ & $\begin{array}{l}5 \\
5 \\
5 \\
5 \\
5 \\
5 \\
5 \\
5 \\
5\end{array}$ & $\begin{array}{l}5 \\
5 \\
5 \\
5 \\
3 \\
3 \\
3 \\
3 \\
3\end{array}$ & $\begin{array}{l}5 \\
5 \\
5 \\
5 \\
5 \\
5 \\
5 \\
5 \\
5\end{array}$ & $\begin{array}{l}5 \\
5 \\
5 \\
5 \\
5 \\
5 \\
5 \\
5 \\
5\end{array}$ \\
\hline Lexan & polyurethane & $\begin{array}{r}0 \\
7 \\
14 \\
21 \\
28 \\
35\end{array}$ & $\begin{array}{l}5 \\
5 \\
5 \\
4 \\
4 \\
-\end{array}$ & $\begin{array}{l}5 \\
5 \\
5 \\
4 \\
4\end{array}$ & $\begin{array}{l}5 \\
5 \\
5 \\
3 \\
1 \\
-\end{array}$ & $\begin{array}{l}5 \\
5 \\
5 \\
3 \\
2\end{array}$ & $\begin{array}{l}5 \\
5 \\
5 \\
5 \\
5 \\
-\end{array}$ & $\begin{array}{l}5 \\
5 \\
5 \\
5 \\
5\end{array}$ \\
\hline Lexan & acrylic & $\begin{array}{r}0 \\
7 \\
14 \\
21 \\
28 \\
35\end{array}$ & $\begin{array}{c}3 \\
3 \\
2-3 \\
2-3 \\
2-3 \\
-\end{array}$ & $\begin{array}{l}4 \\
4 \\
4 \\
4 \\
4\end{array}$ & $\begin{array}{l}3 \\
2 \\
2 \\
1 \\
1 \\
-\end{array}$ & $\begin{array}{l}3 \\
3 \\
3 \\
3 \\
3\end{array}$ & $\begin{array}{l}5 \\
5 \\
5 \\
5 \\
5 \\
-\end{array}$ & $\begin{array}{l}5 \\
5 \\
5 \\
5 \\
5\end{array}$ \\
\hline $\begin{array}{l}\text { Mil. Spec. } \\
\text { PUc } \\
\text { Painted } \\
\text { Steel }\end{array}$ & epoxy 1 & $\begin{array}{r}0 \\
7 \\
14 \\
21 \\
28 \\
35 \\
42 \\
49 \\
56\end{array}$ & $\begin{array}{l}5 \\
4 \\
4 \\
3 \\
3 \\
3 \\
3 \\
3 \\
2\end{array}$ & $\begin{array}{l}5 \\
4 \\
4 \\
3 \\
3 \\
3 \\
3 \\
3 \\
3\end{array}$ & $\begin{array}{l}5 \\
5 \\
3 \\
3 \\
3 \\
3 \\
3 \\
2 \\
2\end{array}$ & $\begin{array}{l}5 \\
5 \\
4 \\
4 \\
4 \\
4 \\
4 \\
4 \\
4\end{array}$ & $\begin{array}{l}5 \\
5 \\
5 \\
5 \\
5 \\
5 \\
5 \\
5 \\
5\end{array}$ & $\begin{array}{l}5 \\
5 \\
5 \\
5 \\
5 \\
5 \\
5 \\
5 \\
5\end{array}$ \\
\hline
\end{tabular}

a See Table 3.8 for a description of the numerical ranking code for assessing tamper tapes.

b Samples were removed from QUV cabinet because their security features were compromised. 
Table 4.2. (Continued)

\begin{tabular}{|c|c|c|c|c|c|c|c|c|}
\hline \multirow[b]{3}{*}{ Surface } & \multirow[b]{3}{*}{ Adhesive } & \multirow[b]{3}{*}{$\begin{array}{l}\text { Days of } \\
\text { Exposure }\end{array}$} & \multicolumn{6}{|c|}{ Condition of Tamper Tape ${ }^{a}$} \\
\hline & & & \multicolumn{2}{|c|}{ Appearance } & \multicolumn{2}{|c|}{ Security Emblem } & \multicolumn{2}{|c|}{ Adhesion } \\
\hline & & & $\begin{array}{c}\text { Window } \\
\text { Area }\end{array}$ & $\begin{array}{l}\text { Vinyl } \\
\text { Area }\end{array}$ & $\begin{array}{c}\text { Window } \\
\text { Area } \\
\end{array}$ & $\begin{array}{l}\text { Vinyl } \\
\text { Area }\end{array}$ & $\begin{array}{c}\text { Window } \\
\text { Area }\end{array}$ & Overall \\
\hline $\begin{array}{l}\text { Mil. Spec. } \\
\text { PU-Painted } \\
\text { Steel }\end{array}$ & epoxy 2 & $\begin{array}{r}0 \\
7 \\
14 \\
21 \\
28 \\
35 \\
42 \\
49 \\
56\end{array}$ & $\begin{array}{l}5 \\
5 \\
5 \\
5 \\
5 \\
5 \\
5 \\
5 \\
4\end{array}$ & $\begin{array}{l}5 \\
5 \\
5 \\
5 \\
5 \\
5 \\
5 \\
5 \\
4\end{array}$ & $\begin{array}{l}5 \\
5 \\
5 \\
5 \\
5 \\
5 \\
5 \\
5 \\
5\end{array}$ & $\begin{array}{l}5 \\
5 \\
5 \\
5 \\
3 \\
3 \\
3 \\
3 \\
3\end{array}$ & $\begin{array}{l}5 \\
5 \\
5 \\
5 \\
5 \\
5 \\
5 \\
5 \\
5\end{array}$ & $\begin{array}{l}5 \\
5 \\
5 \\
5 \\
5 \\
5 \\
5 \\
5 \\
5\end{array}$ \\
\hline $\begin{array}{l}\text { Mil. Spec. } \\
\text { PU-Painted } \\
\text { Steel } \\
\quad\end{array}$ & polyurethane & $\begin{array}{r}0 \\
7 \\
14 \\
21 \\
28 \\
35 \\
42 \\
49 \\
56\end{array}$ & $\begin{array}{l}5 \\
5 \\
4 \\
4 \\
3 \\
2 \\
2 \\
1 \\
1\end{array}$ & $\begin{array}{l}5 \\
5 \\
5 \\
5 \\
4 \\
4 \\
4 \\
4 \\
4\end{array}$ & $\begin{array}{c}5 \\
5 \\
5 \\
3 \\
2 \\
1-2 \\
1-2 \\
1 \\
1\end{array}$ & $\begin{array}{c}5 \\
5 \\
5 \\
4 \\
3-4 \\
3-4 \\
3-4 \\
3-4 \\
3-4\end{array}$ & $\begin{array}{l}5 \\
5 \\
5 \\
5 \\
5 \\
5 \\
5 \\
5 \\
5\end{array}$ & $\begin{array}{l}5 \\
5 \\
5 \\
5 \\
5 \\
5 \\
5 \\
5 \\
5\end{array}$ \\
\hline $\begin{array}{l}\text { Mil. Spec. } \\
\text { PU-Painted } \\
\text { Steel }\end{array}$ & acrylic & $\begin{array}{r}0 \\
7 \\
14 \\
21 \\
28 \\
35\end{array}$ & $\begin{array}{l}3 \\
3 \\
3 \\
3 \\
3 \\
-\mathrm{b}\end{array}$ & $\begin{array}{l}4 \\
4 \\
4 \\
4 \\
4\end{array}$ & $\begin{array}{l}3 \\
2 \\
2 \\
1 \\
1 \\
-\end{array}$ & $\begin{array}{l}3 \\
3 \\
3 \\
3 \\
3\end{array}$ & $\begin{array}{l}5 \\
5 \\
5 \\
5 \\
5 \\
-\end{array}$ & $\begin{array}{l}5 \\
5 \\
5 \\
5 \\
5\end{array}$ \\
\hline
\end{tabular}

a Sec Table 3.8 for a description of the numerical ranking code for assessing tamper tapes.

b Samples were removed from QUV cabinet because their security features were compromised.

c Polyurethane

features were very faded after 4 weeks, while on the other surfaces, it took 7 to 8 weeks for the security features to become badly faded.

Epoxy 2 adhesive-bonded tamper tapes had the best overall performance on all surfaces for the 8 weeks of exposure in the QUV cabinet (Table 4.2). The appearance of the tamper tapes was good, the security feature on all of the tamper tapes remained very visible with only slight fading, and adhesion was excellent.

\subsubsection{Thermal Cycling Exposure Results}

The results of thermal cycling exposure of the $2.54-\mathrm{cm}$-wide Confirm $^{\circledR}$ window tamper tapes bonded to surfaces using the four rapid-set adhesives are given in Table 4.3. The 56-day 
Table 4.3. Evaluation of Tamper Tapes Applied with Candidate Rapid-Set Adhesives and Aged in Thermal Cycling Cabinet $\left(-18^{\circ} \mathrm{C}\right.$ to $\left.46^{\circ} \mathrm{C}\right)$

\begin{tabular}{|c|c|c|c|c|c|c|c|c|}
\hline \multirow[b]{3}{*}{ Surface } & \multirow[b]{3}{*}{ Adhesive } & \multirow[b]{3}{*}{$\begin{array}{c}\text { Days of } \\
\text { Exposure }\end{array}$} & \multicolumn{6}{|c|}{ Condition of Tamper Tape ${ }^{a}$} \\
\hline & & & \multicolumn{2}{|c|}{ Appearance } & \multirow{2}{*}{$\begin{array}{c}\text { Security } \\
\text { Window } \\
\text { Area }\end{array}$} & \multirow{2}{*}{$\begin{array}{c}\text { Emblem } \\
\text { Vinyl } \\
\text { Area }\end{array}$} & \multicolumn{2}{|c|}{ Surface Adhesion } \\
\hline & & & $\begin{array}{c}\text { Window } \\
\text { Area }\end{array}$ & $\begin{array}{l}\text { Vinyl } \\
\text { Area } \\
\end{array}$ & & & $\begin{array}{c}\text { Window } \\
\text { Area }\end{array}$ & Overall \\
\hline Aluminum & epoxy 1 & $\begin{array}{r}0 \\
7 \\
14 \\
21 \\
28 \\
42 \\
56\end{array}$ & $\begin{array}{l}4 \\
4 \\
4 \\
4 \\
4 \\
4 \\
4\end{array}$ & $\begin{array}{l}5 \\
5 \\
5 \\
5 \\
5 \\
5 \\
5\end{array}$ & $\begin{array}{l}5 \\
5 \\
5 \\
5 \\
5 \\
5 \\
5\end{array}$ & $\begin{array}{l}5 \\
5 \\
5 \\
5 \\
5 \\
5 \\
5\end{array}$ & $\begin{array}{l}5 \\
5 \\
5 \\
5 \\
5 \\
5 \\
4\end{array}$ & $\begin{array}{l}5 \\
5 \\
5 \\
5 \\
5 \\
5 \\
4\end{array}$ \\
\hline $\begin{array}{c}\text { Aluminum } \\
.\end{array}$ & epoxy 2 & $\begin{array}{r}0 \\
7 \\
14 \\
21 \\
28 \\
42 \\
56\end{array}$ & $\begin{array}{l}5 \\
5 \\
5 \\
4 \\
4 \\
4 \\
4\end{array}$ & $\begin{array}{l}5 \\
5 \\
5 \\
5 \\
5 \\
5 \\
5\end{array}$ & $\begin{array}{l}5 \\
5 \\
5 \\
5 \\
5 \\
5 \\
5\end{array}$ & $\begin{array}{l}5 \\
5 \\
5 \\
5 \\
5 \\
5 \\
5\end{array}$ & $\begin{array}{l}5 \\
5 \\
5 \\
5 \\
5 \\
5 \\
5\end{array}$ & $\begin{array}{l}5 \\
5 \\
5 \\
5 \\
5 \\
5 \\
5\end{array}$ \\
\hline Aluminum & polyurethane & $\begin{array}{r}0 \\
7 \\
14 \\
21 \\
28 \\
42 \\
56\end{array}$ & $\begin{array}{l}5 \\
5 \\
5 \\
4 \\
4 \\
4 \\
4\end{array}$ & $\begin{array}{l}5 \\
5 \\
5 \\
5 \\
5 \\
5 \\
5\end{array}$ & $\begin{array}{l}5 \\
5 \\
5 \\
5 \\
5 \\
5 \\
5\end{array}$ & $\begin{array}{l}5 \\
5 \\
5 \\
5 \\
5 \\
5 \\
5\end{array}$ & $\begin{array}{l}5 \\
5 \\
5 \\
5 \\
5 \\
5 \\
5\end{array}$ & $\begin{array}{l}5 \\
5 \\
5 \\
5 \\
5 \\
5 \\
5\end{array}$ \\
\hline Aluminum & acrylic & $\begin{array}{r}0 \\
7 \\
14 \\
21 \\
28 \\
42 \\
56\end{array}$ & $\begin{array}{r}4 \\
4 \\
3 \\
3 \\
3 \\
3 \\
3\end{array}$ & $\begin{array}{l}5 \\
5 \\
4 \\
4 \\
4 \\
4 \\
4\end{array}$ & $\begin{array}{l}5 \\
5 \\
5 \\
5 \\
5 \\
4 \\
3\end{array}$ & $\begin{array}{l}5 \\
5 \\
5 \\
5 \\
5 \\
5 \\
5\end{array}$ & $\begin{array}{l}5 \\
5 \\
5 \\
5 \\
5 \\
5 \\
5\end{array}$ & $\begin{array}{l}5 \\
5 \\
5 \\
5 \\
5 \\
5 \\
5\end{array}$ \\
\hline Steel & epoxy 1 & $\begin{array}{r}0 \\
7 \\
14 \\
21 \\
28 \\
42 \\
56\end{array}$ & $\begin{array}{l}5 \\
5 \\
5 \\
4 \\
4 \\
4 \\
4\end{array}$ & $\begin{array}{l}5 \\
5 \\
5 \\
5 \\
5 \\
5 \\
5\end{array}$ & $\begin{array}{r}5 \\
-\quad 5 \\
5 \\
5 \\
5 \\
5 \\
5 \\
. \quad 5\end{array}$ & $\begin{array}{l}5 \\
5 \\
5 \\
5 \\
5 \\
5 \\
5\end{array}$ & $\begin{array}{l}5 \\
5 \\
5 \\
5 \\
5 \\
5 \\
5\end{array}$ & $\begin{array}{l}5 \\
5 \\
5 \\
5 \\
5 \\
5 \\
5\end{array}$ \\
\hline Steel & epoxy 2 & $\begin{array}{r}0 \\
7 \\
14 \\
21 \\
28 \\
42 \\
56\end{array}$ & $\begin{array}{c}5 \\
5 \\
5 \\
4-5 \\
4-5 \\
4-5 \\
4-5\end{array}$ & $\begin{array}{c}5 \\
5 \\
5 \\
4-5 \\
4-5 \\
4-5 \\
4-5\end{array}$ & $\begin{array}{l}5 \\
5 \\
5 \\
5 \\
5 \\
5 \\
5\end{array}$ & $\begin{array}{l}5 \\
5 \\
5 \\
5 \\
5 \\
5 \\
5\end{array}$ & $\begin{array}{l}5 \\
5 \\
5 \\
5 \\
5 \\
5 \\
5\end{array}$ & $\begin{array}{l}5 \\
5 \\
5 \\
5 \\
5 \\
5 \\
5\end{array}$ \\
\hline
\end{tabular}

a See Table 3.8 for a description of the numerical ranking code for assessing tamper tapes. 
Table 4.3. (Continued)

\begin{tabular}{|c|c|c|c|c|c|c|c|c|}
\hline \multirow[b]{3}{*}{ Surface } & \multirow[b]{3}{*}{ Adhesive } & \multirow[b]{3}{*}{$\begin{array}{l}\text { Days of } \\
\text { Exposure }\end{array}$} & \multicolumn{6}{|c|}{ Condition of Tamper Tape ${ }^{a}$} \\
\hline & & & \multicolumn{2}{|c|}{ Appearance } & \multicolumn{2}{|c|}{ Security Emblem } & \multicolumn{2}{|c|}{ Surface Adhesion } \\
\hline & & & $\begin{array}{c}\text { Window } \\
\text { Area }\end{array}$ & $\begin{array}{l}\text { Vinyl } \\
\text { Area }\end{array}$ & $\begin{array}{c}\text { Window } \\
\text { Area }\end{array}$ & $\begin{array}{l}\text { Vinyl } \\
\text { Area }\end{array}$ & $\begin{array}{c}\text { Window } \\
\text { Area }\end{array}$ & Overall \\
\hline Steel & polyurethane & $\begin{array}{r}0 \\
7 \\
14 \\
21 \\
28 \\
42 \\
56\end{array}$ & $\begin{array}{l}5 \\
5 \\
5 \\
5 \\
5 \\
5 \\
4\end{array}$ & $\begin{array}{l}5 \\
5 \\
5 \\
5 \\
5 \\
5 \\
5\end{array}$ & $\begin{array}{l}5 \\
5 \\
5 \\
5 \\
5 \\
5 \\
5\end{array}$ & $\begin{array}{l}5 \\
5 \\
5 \\
5 \\
5 \\
5 \\
5\end{array}$ & $\begin{array}{l}5 \\
5 \\
5 \\
5 \\
5 \\
5 \\
5\end{array}$ & $\begin{array}{l}5 \\
5 \\
5 \\
5 \\
5 \\
5 \\
5\end{array}$ \\
\hline Steel & acrylic & $\begin{array}{r}0 \\
7 \\
14 \\
21 \\
28 \\
42 \\
56\end{array}$ & $\begin{array}{c}4 \\
4 \\
4 \\
3-4 \\
3-4 \\
3-4 \\
3-4\end{array}$ & $\begin{array}{c}5 \\
5 \\
5 \\
4-5 \\
4-5 \\
4-5 \\
4-5\end{array}$ & $\begin{array}{l}5 \\
5 \\
4 \\
4 \\
4 \\
4 \\
4\end{array}$ & $\begin{array}{l}5 \\
5 \\
5 \\
5 \\
5 \\
5 \\
5\end{array}$ & $\begin{array}{l}5 \\
5 \\
5 \\
5 \\
5 \\
5 \\
5\end{array}$ & $\begin{array}{l}5 \\
5 \\
5 \\
5 \\
5 \\
5 \\
5\end{array}$ \\
\hline Wood & epoxy 1 & $\begin{array}{r}0 \\
7 \\
14 \\
21 \\
28 \\
42 \\
56\end{array}$ & $\begin{array}{l}4 \\
4 \\
3 \\
3 \\
3 \\
3 \\
3\end{array}$ & $\begin{array}{l}4-5 \\
4-5 \\
4-5 \\
4-5 \\
4-5 \\
4-5 \\
4-5\end{array}$ & $\begin{array}{l}5 \\
5 \\
5 \\
5 \\
5 \\
5 \\
5\end{array}$ & $\begin{array}{l}5 \\
5 \\
5 \\
5 \\
5 \\
5 \\
5\end{array}$ & $\begin{array}{l}5 \\
5 \\
5 \\
5 \\
5 \\
5 \\
5\end{array}$ & $\begin{array}{l}5 \\
5 \\
5 \\
5 \\
5 \\
5 \\
5\end{array}$ \\
\hline Wood & epoxy 2 & $\begin{array}{r}0 \\
7 \\
14 \\
21 \\
28 \\
42 \\
56\end{array}$ & $\begin{array}{l}5 \\
5 \\
5 \\
5 \\
5 \\
5 \\
5\end{array}$ & $\begin{array}{l}5 \\
5 \\
5 \\
5 \\
5 \\
5 \\
5\end{array}$ & $\begin{array}{l}5 \\
5 \\
5 \\
5 \\
5 \\
5 \\
5\end{array}$ & $\begin{array}{l}5 \\
5 \\
5 \\
5 \\
5 \\
5 \\
5\end{array}$ & $\begin{array}{l}5 \\
5 \\
5 \\
5 \\
5 \\
5 \\
5\end{array}$ & $\begin{array}{l}5 \\
5 \\
5 \\
5 \\
5 \\
5 \\
5\end{array}$ \\
\hline Wood & polyurethane & $\begin{array}{r}0 \\
7 \\
14 \\
21 \\
28 \\
42 \\
56\end{array}$ & $\begin{array}{l}5 \\
5 \\
5 \\
5 \\
5 \\
5 \\
4\end{array}$ & $\begin{array}{l}5 \\
5 \\
5 \\
5 \\
5 \\
5 \\
5\end{array}$ & $\begin{array}{l}5 \\
5 \\
5 \\
5 \\
5 \\
5 \\
5\end{array}$ & $\begin{array}{l}5 \\
5 \\
5 \\
5 \\
5 \\
5 \\
5\end{array}$ & $\begin{array}{l}5 \\
5 \\
5 \\
5 \\
5 \\
5 \\
5\end{array}$ & $\begin{array}{l}5 \\
5 \\
5 \\
5 \\
5 \\
5 \\
5\end{array}$ \\
\hline Wood & acrylic & $\begin{array}{r}0 \\
7 \\
14 \\
21 \\
28 \\
42 \\
56\end{array}$ & $\begin{array}{l}4 \\
4 \\
4 \\
4 \\
4 \\
4 \\
3\end{array}$ & $\begin{array}{l}5 \\
5 \\
5 \\
5 \\
5 \\
5 \\
4\end{array}$ & $\begin{array}{l}5 \\
5 \\
4 \\
4 \\
4 \\
4 \\
4\end{array}$ & $\begin{array}{l}5 \\
5 \\
5 \\
5 \\
5 \\
5 \\
5\end{array}$ & $\begin{array}{l}5 \\
5 \\
5 \\
5 \\
5 \\
5 \\
5\end{array}$ & $\begin{array}{l}5 \\
5 \\
5 \\
5 \\
5 \\
5 \\
5\end{array}$ \\
\hline
\end{tabular}

a Sec Table 3.8 for a description of the numerical ranking code for assessing tamper tapes. 
Table 4.3. (Continued)

\begin{tabular}{|c|c|c|c|c|c|c|c|c|}
\hline \multirow[b]{3}{*}{ Surface } & \multirow[b]{3}{*}{ Adhesive } & \multirow[b]{3}{*}{$\begin{array}{c}\text { Days of } \\
\text { Exposure }\end{array}$} & \multicolumn{6}{|c|}{ Condition of Tamper Tape ${ }^{a}$} \\
\hline & & & \multicolumn{2}{|c|}{ Appearance } & \multicolumn{2}{|c|}{ Security Emblem } & \multicolumn{2}{|c|}{ Surface Adhesion } \\
\hline & & & $\begin{array}{c}\text { Window } \\
\text { Area }\end{array}$ & $\begin{array}{c}\text { Vinyl } \\
\text { Area } \\
\end{array}$ & $\begin{array}{c}\text { Window } \\
\text { Area }\end{array}$ & $\begin{array}{l}\text { Vinyl } \\
\text { Area }\end{array}$ & $\begin{array}{c}\text { Window } \\
\text { Area }\end{array}$ & Overall \\
\hline $\begin{array}{l}\text { Fiberglass } \\
\text { Board }\end{array}$ & epoxy 1 & $\begin{array}{r}0 \\
7 \\
14 \\
21 \\
28 \\
42 \\
56\end{array}$ & $\begin{array}{l}5 \\
5 \\
5 \\
5 \\
5 \\
5 \\
5\end{array}$ & $\begin{array}{l}5 \\
5 \\
5 \\
5 \\
5 \\
5 \\
5\end{array}$ & $\begin{array}{r}5 \\
5 \\
5 \\
5 \\
5 \\
5 \\
5 \\
5\end{array}$ & $\begin{array}{l}5 \\
5 \\
5 \\
5 \\
5 \\
5 \\
5\end{array}$ & $\begin{array}{l}5 \\
5 \\
5 . \\
5 \\
5 \\
5 \\
5\end{array}$ & $\begin{array}{l}5 \\
5 \\
5 \\
5 \\
5 \\
5 \\
5\end{array}$ \\
\hline $\begin{array}{l}\text { Fiberglass } \\
\text { Board. }\end{array}$ & epoxy 2 & $\begin{array}{r}0 \\
7 \\
14 \\
21 \\
28 \\
42 \\
56\end{array}$ & $\begin{array}{l}.5 \\
5 \\
5 \\
5 \\
5 \\
5 \\
5\end{array}$ & $\begin{array}{l}5 \\
5 \\
5 \\
5 \\
5 \\
5 \\
5\end{array}$ & $\begin{array}{l}5 \\
5 \\
5 \\
5 \\
5 \\
5 \\
5\end{array}$ & $\begin{array}{l}5 \\
5 \\
5 \\
5 \\
5 \\
5 \\
5\end{array}$ & $\begin{array}{r}5 \\
5 \\
5 \\
5 \\
5 \\
5 \\
5\end{array}$ & $\begin{array}{l}5 \\
5 \\
5 \\
5 \\
5 \\
5 \\
5\end{array}$ \\
\hline $\begin{array}{l}\text { Fiberglass } \\
\text { Board }\end{array}$ & polyurethane & $\begin{array}{r}0 \\
7 \\
14 \\
21 \\
28 \\
42 \\
56\end{array}$ & $\begin{array}{l}5 \\
5 \\
5 \\
5 \\
5 \\
5 \\
4\end{array}$ & $\begin{array}{l}5 \\
5 \\
5 \\
5 \\
5 \\
5 \\
5\end{array}$ & $\begin{array}{r}5 \\
5 \\
5 \\
.5 \\
5 \\
5 \\
5 \\
5\end{array}$ & $\begin{array}{l}5 \\
5 \\
5 \\
5 \\
5 \\
5 \\
5\end{array}$ & $\begin{array}{l}5 \\
5 \\
5 \\
5 \\
5 \\
5 \\
5\end{array}$ & $\begin{array}{l}5 \\
5 \\
5 \\
5 \\
5 \\
5 \\
5\end{array}$ \\
\hline $\begin{array}{l}\text { Fiberglass } \\
\text { Board }\end{array}$ & acrylic & $\begin{array}{r}0 \\
7 \\
14 \\
21 \\
28 \\
42 \\
56\end{array}$ & $\begin{array}{l}4 \\
4 \\
4 \\
4 \\
4 \\
4 \\
4\end{array}$ & $\begin{array}{c}5 \\
5 \\
4-5 \\
4-5 \\
4-5 \\
4-5 \\
4-5\end{array}$ & $\begin{array}{l}5 \\
5 \\
4 \\
4 \\
4 \\
4 \\
4\end{array}$ & $\begin{array}{l}5 \\
5 \\
5 \\
5 \\
5 \\
5 \\
5\end{array}$ & $\begin{array}{l}5 \\
5 \\
5 \\
5 \\
5 \\
5 \\
5\end{array}$ & $\begin{array}{l}5 \\
5 \\
5 \\
5 \\
5 \\
5 \\
5\end{array}$ \\
\hline Lexan & epoxy 1 & $\begin{array}{r}0 \\
7 \\
14 \\
21 \\
28 \\
42 \\
56\end{array}$ & $\begin{array}{l}5 \\
5 \\
5 \\
5 \\
5 \\
5 \\
5\end{array}$ & $\begin{array}{l}5 \\
5 \\
5 \\
5 \\
5 \\
5 \\
5\end{array}$ & $\begin{array}{l}5 \\
5 \\
5 \\
5 \\
5 \\
5 \\
5\end{array}$ & $\begin{array}{l}5 \\
5 \\
5 \\
5 \\
5 \\
5 \\
5\end{array}$ & $\begin{array}{l}5 \\
5 \\
5 \\
5 \\
5 \\
5 \\
5\end{array}$ & $\begin{array}{l}5 \\
5 \\
5 \\
5 \\
5 \\
5 \\
5\end{array}$ \\
\hline Lexan & epoxy 2 & $\begin{array}{r}0 \\
7 \\
14 \\
21 \\
28 \\
42 \\
56\end{array}$ & $\begin{array}{l}5 \\
5 \\
5 \\
5 \\
5 \\
5 \\
5\end{array}$ & $\begin{array}{l}5 \\
5 \\
5 \\
5 \\
5 \\
5 \\
5\end{array}$ & $\begin{array}{l}5 \\
5 \\
5 \\
5 \\
5 \\
5 \\
5\end{array}$ & $\begin{array}{l}5 \\
5 \\
5 \\
5 \\
5 \\
5 \\
5\end{array}$ & $\begin{array}{l}5 \\
5 \\
5 \\
5 \\
5 \\
5 \\
5\end{array}$ & $\begin{array}{l}5 \\
5 \\
5 \\
5 \\
5 \\
5 \\
5 \\
5\end{array}$ \\
\hline
\end{tabular}

a See Table 3.8 for a description of the numerical ranking code for assessing tamper tapes. 


\section{Table 4.3. (Continued)}

\begin{tabular}{|c|c|c|c|c|c|c|c|c|}
\hline \multirow[b]{3}{*}{ Surface } & \multirow[b]{3}{*}{ Adhesive } & \multirow[b]{3}{*}{$\begin{array}{c}\text { Days of } \\
\text { Exposure }\end{array}$} & \multicolumn{6}{|c|}{ Condition of Tamper Tape ${ }^{a}$} \\
\hline & & & \multicolumn{2}{|c|}{ Appearance } & \multicolumn{2}{|c|}{ Security Emblem } & \multicolumn{2}{|c|}{ Surface Adhesion } \\
\hline & & & $\begin{array}{c}\text { Window } \\
\text { Area }\end{array}$ & $\begin{array}{l}\text { Vinyl } \\
\text { Area }\end{array}$ & $\begin{array}{c}\text { Window } \\
\text { Area }\end{array}$ & $\begin{array}{l}\text { Vinyl } \\
\text { Area }\end{array}$ & $\begin{array}{c}\text { Window } \\
\text { Area }\end{array}$ & Overall \\
\hline Lexan & polyurethane & $\begin{array}{r}0 \\
7 \\
14 \\
21 \\
28 \\
42 \\
56\end{array}$ & $\begin{array}{l}5 \\
5 \\
5 \\
5 \\
5 \\
5 \\
4\end{array}$ & $\begin{array}{l}5 \\
5 \\
5 \\
5 \\
5 \\
5 \\
5\end{array}$ & $\begin{array}{l}5 \\
5 \\
5 \\
5 \\
5 \\
5 \\
5\end{array}$ & $\begin{array}{l}5 \\
5 \\
5 \\
5 \\
5 \\
5 \\
5\end{array}$ & $\begin{array}{l}5 \\
5 \\
5 \\
5 \\
5 \\
5 \\
5\end{array}$ & $\begin{array}{l}5 \\
5 \\
5 \\
5 \\
5 \\
5 \\
5\end{array}$ \\
\hline Lexan & acrylic & $\begin{array}{r}0 \\
7 \\
14 \\
21 \\
28 \\
42 \\
56\end{array}$ & $\begin{array}{l}4 \\
4 \\
4 \\
4 \\
4 \\
4 \\
4\end{array}$ & $\begin{array}{l}5 \\
5 \\
5 \\
5 \\
5 \\
5 \\
5\end{array}$ & $\begin{array}{l}5 \\
5 \\
4 \\
4 \\
4 \\
3 \\
3\end{array}$ & $\begin{array}{l}5 \\
5 \\
5 \\
5 \\
5 \\
5 \\
5\end{array}$ & $\begin{array}{l}5 \\
5 \\
5 \\
5 \\
5 \\
5 \\
5\end{array}$ & $\begin{array}{l}5 \\
5 \\
5 \\
5 \\
5 \\
5 \\
5\end{array}$ \\
\hline $\begin{array}{l}\text { Mil. Spec. } \\
\text { PUb } \\
\text { Painted } \\
\text { Steel }\end{array}$ & epoxy 1 & $\begin{array}{r}0 \\
7 \\
14 \\
21 \\
28 \\
42 \\
56\end{array}$ & $\begin{array}{l}5 \\
5 \\
5 \\
5 \\
5 \\
5 \\
4\end{array}$ & $\begin{array}{l}5 \\
5 \\
5 \\
5 \\
5 \\
5 \\
5\end{array}$ & $\begin{array}{l}5 \\
5 \\
5 \\
5 \\
5 \\
5 \\
5\end{array}$ & $\begin{array}{l}5 \\
5 \\
5 \\
5 \\
5 \\
5 \\
5\end{array}$ & $\begin{array}{l}5 \\
5 \\
5 \\
5 \\
5 \\
5 \\
5\end{array}$ & $\begin{array}{l}5 \\
5 \\
5 \\
5 \\
5 \\
5 \\
5\end{array}$ \\
\hline $\begin{array}{l}\text { Mil. Spec. } \\
\text { PU-Painted } \\
\text { Steel }\end{array}$ & cpoxy 2 & $\begin{array}{r}0 \\
7 \\
14 \\
21 \\
28 \\
42 \\
56\end{array}$ & $\begin{array}{l}5 \\
5 \\
5 \\
5 \\
5 \\
5 \\
5\end{array}$ & $\begin{array}{l}5 \\
5 \\
5 \\
5 \\
5 \\
5 \\
5\end{array}$ & $\begin{array}{l}5 \\
5 \\
5 \\
5 \\
5 \\
5 \\
5\end{array}$ & $\begin{array}{l}5 \\
5 \\
5 \\
5 \\
5 \\
5 \\
5\end{array}$ & $\begin{array}{l}5 \\
5 \\
5 \\
5 \\
5 \\
5 \\
5\end{array}$ & $\begin{array}{l}5 \\
5 \\
5 \\
5 \\
5 \\
5 \\
5\end{array}$ \\
\hline $\begin{array}{l}\text { Mil. Spec. } \\
\text { PU-Painted } \\
\text { Steel }\end{array}$ & polyurethane & $\begin{array}{r}0 \\
7 \\
14 \\
21 \\
28 \\
42 \\
56\end{array}$ & $\begin{array}{l}5 \\
5 \\
5 \\
5 \\
5 \\
5 \\
4\end{array}$ & $\begin{array}{l}5 \\
5 \\
5 \\
5 \\
5 \\
5 \\
5\end{array}$ & $\begin{array}{l}5 \\
5 \\
5 \\
5 \\
5 \\
5 \\
5\end{array}$ & $\begin{array}{l}5 \\
5 \\
5 \\
5 \\
5 \\
5 \\
5\end{array}$ & $\begin{array}{l}5 \\
5 \\
5 \\
5 \\
5 \\
5 \\
5\end{array}$ & $\begin{array}{l}5 \\
5 \\
5 \\
5 \\
5 \\
5 \\
5\end{array}$ \\
\hline $\begin{array}{l}\text { Mil. Spec. } \\
\text { PU-Painted } \\
\text { Steel }\end{array}$ & acrylic & $\begin{array}{r}0 \\
7 \\
14 \\
21 \\
28 \\
42 \\
56\end{array}$ & $\begin{array}{l}4 \\
4 \\
4 \\
4 \\
4 \\
3 \\
3\end{array}$ & $\begin{array}{c}5 \\
5 \\
4-5 \\
4-5 \\
4-5 \\
4 \\
4\end{array}$ & $\begin{array}{l}5 \\
5 \\
4 \\
4 \\
4 \\
4 \\
3\end{array}$ & $\begin{array}{l}5 \\
5 \\
5 \\
5 \\
5 \\
5 \\
5\end{array}$ & $\begin{array}{l}5 \\
5 \\
5 \\
5 \\
5 \\
5 \\
5\end{array}$ & $\begin{array}{l}5 \\
5 \\
5 \\
5 \\
5 \\
5 \\
5\end{array}$ \\
\hline
\end{tabular}

\footnotetext{
a See Table 3.8 for a description of the numerical ranking code for assessing tamper tapes.

$b$ Polyurethanc
} 
exposure to cycles of $-18^{\circ} \mathrm{C}$ to $46^{\circ} \mathrm{C}$ showed little or no effects on the security features of the tamper tapes. The acrylic-bonded tamper tapes did show slight to moderate fading of their security feature during the exposure period. The tamper tapes displayed excellent adhesion to the surfaces. The appearance of the tamper tapes using the acrylic and epoxy 1 adhesives changed slightly. Overall, the tamper tapes held up very well to thermal cycling.

\subsubsection{Control Exposure Results}

The results for the tamper tapes held under the control exposure conditions $\left(23^{\circ} \mathrm{C}, 50 \%\right.$ relative humidity) are given in Table 4.4. These rapid-set adhesive-bonded tamper tapes showed no change in appearance, adhesion, or security features during the seven weeks of storage.

\subsection{WEATHERING OF TAMPER TAPES MADE WITH THREE DIFFERENT CONFIRM® PRODUCTS}

In the second study using rapid-set adhesives, tamper tapes with $2.54-\mathrm{cm}$-wide Confirm ${ }^{\circledR}$ windows were made in the laboratory to determine if another $3 \mathrm{M}$ Confirm ${ }^{\circledR}$ material might perform better than the 1700 series previously used in all other weathering studies. Three Confirm $^{\circledR}$ materials, i.e., Confirm ${ }^{\circledR} 1500$ with primer, Confirm $1500^{\circledR}$ without primer, and Confirm $1300^{\circledR}$, were used. The primary differences between these $3 \mathrm{M}$ products were in the bead-bond layers. The 1700 products contain an "alkyd" layer, the 1500 products a polyurethane layer, and the 1300 product a "latex" layer. The tamper tapes were made in the laboratory, as described for the first study with rapid-set adhesives, by bonding the various Confirm ${ }^{\circledR}$ materials to vinyl underlay using a 3M Company PSA. The same four candidate rapid-set adhesives, i.e., epoxy 1 , epoxy 2, polyurethane, and acrylic, were used to bond the prototype tamper tapes to four surfaces, i.e., roughened aluminum, roughened steel, wood, and Mil. Spec. polyurethane-painted steel. They were tested at Daytona Beach, Florida, in the QUV cabinet (ultraviolet light, $60^{\circ} \mathrm{C}$; condensing humidity, $40^{\circ} \mathrm{C}$ ), and in the thermal cycling cabinet $\left(-18^{\circ} \mathrm{C}\right.$ to $\left.46^{\circ} \mathrm{C}\right)$. After exposure, the tamper tapes were examined as was done in the first study using rapid-set adhesives.

\subsubsection{Florida Exposure Results}

The results from the exposure of the various Confirm ${ }^{\circledR}$ material tamper tapes in Daytona Beach, Florida are given in Table 4.5. Over 2 months, there was little to no change in the adhesion of the tamper tapes to the various surfaces. However, significant changes occurred in the security

feature of the tamper tapes. The security feature on the tamper tapes prepared with Confirm ${ }^{\circledR} 1500$ (with and without a primer) turned black, an effect not noted previously with other prototype tamper tapes. This change probably was due to corrosion of the reflective layer under the glass 
Table 4.4. Evaluation of Tamper Tapes Applied with Candidate Rapid-Set Adhesives Held Under Control Conditions $\left(23^{\circ} \mathrm{C}, 50 \%\right.$ Relative Humidity)

\begin{tabular}{|c|c|c|c|c|c|c|c|c|}
\hline \multirow[b]{3}{*}{ Surface } & \multirow[b]{3}{*}{ Adhesive } & \multirow[b]{3}{*}{$\begin{array}{c}\text { Days of } \\
\text { Exposure }\end{array}$} & \multicolumn{6}{|c|}{ Condition of Tamper Tape ${ }^{a}$} \\
\hline & & & \multicolumn{2}{|c|}{ Appearance } & \multirow{2}{*}{$\begin{array}{l}\text { Security } \\
\text { Window } \\
\text { Area }\end{array}$} & \multirow{2}{*}{$\begin{array}{c}\text { Emblem } \\
\text { Vinyl } \\
\text { Area } \\
\end{array}$} & \multicolumn{2}{|c|}{ Surface Adhesion } \\
\hline & & & $\begin{array}{c}\text { Window } \\
\text { Area }\end{array}$ & $\begin{array}{l}\text { Vinyl } \\
\text { Area }\end{array}$ & & & $\begin{array}{c}\text { Window } \\
\text { Area }\end{array}$ & Overall \\
\hline \multirow[t]{5}{*}{ Aluminum } & $\begin{array}{c}\text { epoxy } 1 \\
\text { epoxy } 2 \\
\text { polyurethane } \\
\text { acrylic }\end{array}$ & 7 & $\begin{array}{c}5 \\
5 \\
4-5 \\
4\end{array}$ & $\begin{array}{c}5 \\
5 \\
4-5 \\
5\end{array}$ & $\begin{array}{l}5 \\
5 \\
5 \\
5\end{array}$ & $\begin{array}{l}5 \\
5 \\
5 \\
5\end{array}$ & $\begin{array}{l}5 \\
5 \\
5 \\
5\end{array}$ & $\begin{array}{l}5 \\
5 \\
5 \\
5\end{array}$ \\
\hline & $\begin{array}{c}\text { epoxy } 1 \\
\text { epoxy } 2 \\
\text { polyurethane } \\
\text { acrylic }\end{array}$ & 21 & $\begin{array}{c}5 \\
5 \\
4-5 \\
4\end{array}$ & $\begin{array}{c}5 \\
5 \\
4-5 \\
5\end{array}$ & $\begin{array}{l}5 \\
5 \\
5 \\
5\end{array}$ & $\begin{array}{l}5 \\
5 \\
5 \\
5\end{array}$ & $\begin{array}{l}5 \\
5 \\
5 \\
5\end{array}$ & $\begin{array}{l}5 \\
5 \\
5 \\
5\end{array}$ \\
\hline & $\begin{array}{c}\text { epoxy } 1 \\
\text { epoxy } 2 \\
\text { polyurethanc } \\
\text { acrylic }\end{array}$ & 35 & $\begin{array}{c}5 \\
5 \\
4-5 \\
4\end{array}$ & $\begin{array}{c}5 \\
5 \\
4-5 \\
5\end{array}$ & $\begin{array}{l}5 \\
5 \\
5 \\
5\end{array}$ & $\begin{array}{l}5 \\
5 \\
5 \\
5\end{array}$ & $\begin{array}{l}5 \\
5 \\
5 \\
5\end{array}$ & $\begin{array}{l}5 \\
5 \\
5 \\
5\end{array}$ \\
\hline & $\begin{array}{c}\text { epoxy } 1 \\
\text { epoxy } 2 \\
\text { polyurethane } \\
\text { acrylic }\end{array}$ & 49 & $\begin{array}{c}5 \\
5 \\
4-5 \\
4\end{array}$ & $\begin{array}{l}5 \\
5 \\
5 \\
5\end{array}$ & $\begin{array}{l}5 \\
5 \\
5 \\
5\end{array}$ & $\begin{array}{l}5 \\
5 \\
5 \\
5\end{array}$ & $\begin{array}{l}5 \\
5 \\
5 \\
5\end{array}$ & $\begin{array}{l}5 \\
5 \\
5 \\
5\end{array}$ \\
\hline & $\begin{array}{c}\text { epoxy } 1 \\
\text { epoxy } 2 \\
\text { polyurcthane } \\
\text { acrylic }\end{array}$ & 63 & $\begin{array}{c}5 \\
5 \\
4-5 \\
4\end{array}$ & $\begin{array}{l}5 \\
5 \\
5 \\
5\end{array}$ & $\begin{array}{l}5 \\
5 \\
5 \\
5\end{array}$ & $\begin{array}{l}5 \\
5 \\
5 \\
5\end{array}$ & $\begin{array}{l}5 \\
5 \\
5 \\
5\end{array}$ & $\begin{array}{l}5 \\
5 \\
5 \\
5\end{array}$ \\
\hline \multirow[t]{5}{*}{ Steel } & $\begin{array}{c}\text { epoxy } 1 \\
\text { epoxy } 2 \\
\text { polyurethane } \\
\text { acrylic }\end{array}$ & 7 & $\begin{array}{c}4-5 \\
5 \\
4-5 \\
4\end{array}$ & $\begin{array}{c}4-5 \\
5 \\
4-5 \\
5\end{array}$ & $\begin{array}{l}5 \\
5 \\
5 \\
5\end{array}$ & $\begin{array}{l}5 \\
5 \\
5 \\
5\end{array}$ & $\begin{array}{l}5 \\
5 \\
5 \\
5\end{array}$ & $\begin{array}{l}5 \\
5 \\
5 \\
5\end{array}$ \\
\hline & $\begin{array}{c}\text { epoxy } 1 \\
\text { epoxy } 2 \\
\text { polyurethane } \\
\text { acrylic }\end{array}$ & 21 & $\begin{array}{c}4-5 \\
5 \\
4-5 \\
4\end{array}$ & $\begin{array}{c}4-5 \\
5 \\
4-5 \\
5\end{array}$ & $\begin{array}{l}5 \\
5 \\
5 \\
5\end{array}$ & $\begin{array}{l}5 \\
5 \\
5 \\
5\end{array}$ & $\begin{array}{l}5 \\
5 \\
5 \\
5\end{array}$ & $\begin{array}{l}5 \\
5 \\
5 \\
5\end{array}$ \\
\hline & $\begin{array}{c}\text { epoxy } 1 \\
\text { epoxy } 2 \\
\text { polyurethane } \\
\text { acrylic }\end{array}$ & 35 & $\begin{array}{c}4-5 \\
5 \\
4-5 \\
4\end{array}$ & $\begin{array}{c}4-5 \\
5 \\
4-5 \\
5\end{array}$ & $\begin{array}{l}5 \\
5 \\
5 \\
5\end{array}$ & $\begin{array}{l}5 \\
5 \\
5 \\
5\end{array}$ & $\begin{array}{l}5 \\
5 \\
5 \\
5\end{array}$ & $\begin{array}{l}5 \\
5 \\
5 \\
5\end{array}$ \\
\hline & $\begin{array}{c}\text { epoxy } 1 \\
\text { epoxy } 2 \\
\text { polyurethane } \\
\text { acrylic }\end{array}$ & 49 & $\begin{array}{c}4-5 \\
5 \\
4-5 \\
4\end{array}$ & $\begin{array}{l}4-5 \\
5 \\
4-5 \\
4-5\end{array}$ & $\begin{array}{l}5 \\
5 \\
5 \\
5\end{array}$ & $\begin{array}{l}5 \\
5 \\
5 \\
5\end{array}$ & $\begin{array}{l}5 \\
5 \\
5 \\
5\end{array}$ & $\begin{array}{l}5 \\
5 \\
5 \\
5\end{array}$ \\
\hline & $\begin{array}{c}\text { epoxy } 1 \\
\text { epoxy } 2 \\
\text { polyurcthane } \\
\text { acrylic }\end{array}$ & 63 & $\begin{array}{c}4-5 \\
5 \\
4-5 \\
4\end{array}$ & $\begin{array}{c}5 \\
5 \\
4-5 \\
4-5\end{array}$ & $\begin{array}{l}5 \\
5 \\
5 \\
5\end{array}$ & $\begin{array}{l}5 \\
5 \\
5 \\
5\end{array}$ & $\begin{array}{l}5 \\
5 \\
5 \\
5\end{array}$ & $\begin{array}{l}5 \\
5 \\
5 \\
5\end{array}$ \\
\hline
\end{tabular}

a Sec Table 3.8 for a description of the numcrical ranking code for assessing tamper tapes. 
Table 4.4. (Continued)

\begin{tabular}{|c|c|c|c|c|c|c|c|c|}
\hline \multirow[b]{3}{*}{ Surface } & \multirow[b]{3}{*}{ Adhesive } & \multirow[b]{3}{*}{$\begin{array}{c}\text { Days of } \\
\text { Exposure }\end{array}$} & \multicolumn{6}{|c|}{ Condition of Tamper Tape ${ }^{a}$} \\
\hline & & & \multicolumn{2}{|c|}{ Appearance } & \multicolumn{2}{|c|}{ Security Emblem } & \multicolumn{2}{|c|}{ Surface Adhesion } \\
\hline & & & $\begin{array}{c}\text { Window } \\
\text { Area } \\
\end{array}$ & $\begin{array}{l}\text { Vinyl } \\
\text { Area } \\
\end{array}$ & $\begin{array}{c}\text { Window } \\
\text { Area }\end{array}$ & $\begin{array}{l}\text { Vinyl } \\
\text { Area }\end{array}$ & $\begin{array}{c}\text { Window } \\
\text { Area } \\
\end{array}$ & Overall \\
\hline \multirow[t]{5}{*}{ Wood } & $\begin{array}{c}\text { epoxy } 1 \\
\text { epoxy } 2 \\
\text { polyurethane } \\
\text { acrylic }\end{array}$ & 7 & $\begin{array}{l}5 \\
5 \\
5 \\
4\end{array}$ & $\begin{array}{l}5 \\
5 \\
5 \\
5\end{array}$ & $\begin{array}{l}5 \\
5 \\
5 \\
4\end{array}$ & $\begin{array}{l}5 \\
5 \\
5 \\
5\end{array}$ & $\begin{array}{l}5 \\
5 \\
5 \\
5\end{array}$ & $\begin{array}{l}5 \\
5 \\
5 \\
5\end{array}$ \\
\hline & $\begin{array}{c}\text { epoxy } 1 \\
\text { epoxy } 2 \\
\text { polyurethane } \\
\text { acrylic }\end{array}$ & $\begin{array}{c}21 \\
.\end{array}$ & $\begin{array}{l}5 \\
5 \\
5 \\
4\end{array}$ & $\begin{array}{l}5 \\
5 \\
5 \\
5\end{array}$ & $\begin{array}{l}5 \\
5 \\
5 \\
4\end{array}$ & $\begin{array}{l}5 \\
5 \\
5 \\
5\end{array}$ & $\begin{array}{l}5 \\
5 \\
5 \\
5\end{array}$ & $\begin{array}{l}5 \\
5 \\
5 \\
5\end{array}$ \\
\hline & $\begin{array}{c}\text { epoxy } 1 \\
\text { epoxy } 2 \\
\text { polyurethane } \\
\text { acrylic }\end{array}$ & 35 & $\begin{array}{l}5 \\
5 \\
5 \\
4\end{array}$ & $\begin{array}{l}5 \\
5 \\
5 \\
5\end{array}$ & $\begin{array}{l}5 \\
5 \\
5 \\
4\end{array}$ & $\begin{array}{l}5 \\
5 \\
5 \\
5\end{array}$ & $\begin{array}{l}5 \\
5 \\
5 \\
5\end{array}$ & $\begin{array}{l}5 \\
5 \\
5 \\
5\end{array}$ \\
\hline & $\begin{array}{c}\text { epoxy } 1 \\
\text { epoxy } 2 \\
\text { polyurethane } \\
\text { acrylic }\end{array}$ & 49 & $\begin{array}{c}5 \\
5 \\
4-5 \\
4\end{array}$ & $\begin{array}{l}5 \\
5 \\
5 \\
5\end{array}$ & $\begin{array}{l}5 \\
5 \\
5 \\
4\end{array}$ & $\begin{array}{l}5 \\
5 \\
5 \\
5\end{array}$ & $\begin{array}{l}5 \\
5 \\
5 \\
5\end{array}$ & $\begin{array}{l}5 \\
5 \\
5 \\
5\end{array}$ \\
\hline & $\begin{array}{c}\text { epoxy } 1 \\
\text { epoxy } 2 \\
\text { polyurethane } \\
\text { acrylic }\end{array}$ & 63 & $\begin{array}{c}5 \\
5 \\
4-5 \\
4\end{array}$ & $\begin{array}{l}5 \\
5 \\
5 \\
5\end{array}$ & $\begin{array}{l}5 \\
5 \\
5 \\
4\end{array}$ & $\begin{array}{l}5 \\
5 \\
5 \\
5\end{array}$ & $\begin{array}{l}5 \\
5 \\
5 \\
5\end{array}$ & $\begin{array}{l}5 \\
5 \\
5 \\
5\end{array}$ \\
\hline \multirow[t]{5}{*}{$\begin{array}{l}\text { Polyester } \\
\text { Fiberglass } \\
\text { Board }\end{array}$} & $\begin{array}{l}\text { epoxy } 1 \\
\text { epoxy } 2 \\
\text { polyurethane } \\
\text { acrylic }\end{array}$ & 7 & $\begin{array}{c}4-5 \\
5 \\
5 \\
4\end{array}$ & $\begin{array}{l}5 \\
5 \\
5 \\
5\end{array}$ & $\begin{array}{l}5 \\
5 \\
5 \\
5\end{array}$ & $\begin{array}{l}5 \\
5 \\
5 \\
5\end{array}$ & $\begin{array}{l}5 \\
5 \\
5 \\
5\end{array}$ & $\begin{array}{l}5 \\
5 \\
5 \\
5\end{array}$ \\
\hline & $\begin{array}{c}\text { epoxy } 1 \\
\text { epoxy } 2 \\
\text { polyurethane } \\
\text { acrylic }\end{array}$ & 21 & $\begin{array}{c}4-5 \\
5 \\
5 \\
4\end{array}$ & $\begin{array}{l}5 \\
5 \\
5 \\
5\end{array}$ & $\begin{array}{l}5 \\
5 \\
5 \\
5\end{array}$ & $\begin{array}{l}5 \\
5 \\
5 \\
5\end{array}$ & $\begin{array}{l}5 \\
5 \\
5 \\
5\end{array}$ & $\begin{array}{l}5 \\
5 \\
5 \\
5\end{array}$ \\
\hline & $\begin{array}{c}\text { epoxy } 1 \\
\text { epoxy } 2 \\
\text { polyurethane } \\
\text { acrylic }\end{array}$ & 35 & $\begin{array}{l}4-5 \\
5 \\
5 \\
4\end{array}$ & $\begin{array}{l}5 \\
5 \\
5 \\
5\end{array}$ & $\begin{array}{l}5 \\
5 \\
5 \\
5\end{array}$ & $\begin{array}{l}5 \\
5 \\
5 \\
5\end{array}$ & $\begin{array}{l}5 \\
5 \\
5 \\
5\end{array}$ & $\begin{array}{l}5 \\
5 \\
5 \\
5\end{array}$ \\
\hline & $\begin{array}{c}\text { epoxy } 1 \\
\text { epoxy } 2 \\
\text { polyurethane } \\
\text { acrylic }\end{array}$ & 49 & $\begin{array}{c}4-5 \\
5 \\
4-5 \\
4\end{array}$ & $\begin{array}{l}5 \\
5 \\
5 \\
5\end{array}$ & $\begin{array}{l}5 \\
5 \\
5 \\
5\end{array}$ & $\begin{array}{l}5 \\
5 \\
5 \\
5\end{array}$ & $\begin{array}{l}5 \\
5 \\
5 \\
5\end{array}$ & $\begin{array}{l}5 \\
5 \\
5 \\
5\end{array}$ \\
\hline & $\begin{array}{c}\text { epoxy } 1 \\
\text { epoxy } 2 \\
\text { polyurethane } \\
\text { acrylic }\end{array}$ & 63 & $\begin{array}{l}5 \\
5 \\
5 \\
4 \\
\end{array}$ & $\begin{array}{l}5 \\
5 \\
5 \\
5 \\
\end{array}$ & $\begin{array}{l}5 \\
5 \\
5 \\
4 \\
\end{array}$ & $\begin{array}{l}5 \\
5 \\
5 \\
5\end{array}$ & $\begin{array}{l}5 \\
5 \\
5 \\
5 \\
\end{array}$ & $\begin{array}{l}5 \\
5 \\
5 \\
5 \\
\end{array}$ \\
\hline
\end{tabular}

a See Table 3.8 for a description of the numerical ranking code for assessing tamper tapes. 
Table 4.4. (Continued)

\begin{tabular}{|c|c|c|c|c|c|c|c|c|}
\hline \multirow[b]{3}{*}{ Surface } & \multirow[b]{3}{*}{ Adhesive } & \multirow[b]{3}{*}{$\begin{array}{c}\text { Days of } \\
\text { Exposure }\end{array}$} & \multicolumn{6}{|c|}{ Condition of Tamper Tape ${ }^{a}$} \\
\hline & & & \multicolumn{2}{|c|}{ Appearance } & \multicolumn{2}{|c|}{ Security Emblem } & \multicolumn{2}{|c|}{ Surface Adhesion } \\
\hline & & & $\begin{array}{c}\text { Window } \\
\text { Area }\end{array}$ & $\begin{array}{l}\text { Vinyl } \\
\text { Area }\end{array}$ & $\begin{array}{c}\text { Window } \\
\text { Area }\end{array}$ & $\begin{array}{l}\text { Vinyl } \\
\text { Area } \\
\end{array}$ & $\begin{array}{c}\text { Window } \\
\text { Area }\end{array}$ & Overall \\
\hline \multirow[t]{5}{*}{ Lexan } & $\begin{array}{c}\text { epoxy } 1 \\
\text { epoxy } 2 \\
\text { polyurethane } \\
\text { acrylic }\end{array}$ & 7 & $\begin{array}{l}5 \\
5 \\
5 \\
3\end{array}$ & $\begin{array}{l}5 \\
5 \\
5 \\
5\end{array}$ & $\begin{array}{l}5 \\
5 \\
5 \\
2\end{array}$ & $\begin{array}{l}5 \\
5 \\
5 \\
5\end{array}$ & $\begin{array}{l}5 \\
5 \\
5 \\
5\end{array}$ & $\begin{array}{l}5 \\
5 \\
5 \\
5\end{array}$ \\
\hline & $\begin{array}{c}\text { epoxy } 1 \\
\text { epoxy } 2 \\
\text { polyurethane } \\
\text { acrylic }\end{array}$ & 21 & $\begin{array}{l}5 \\
5 \\
5 \\
3\end{array}$ & $\begin{array}{l}5 \\
5 \\
5 \\
5\end{array}$ & $\begin{array}{l}5 \\
5 \\
5 \\
2\end{array}$ & $\begin{array}{l}5 \\
5 \\
5 \\
5\end{array}$ & $\begin{array}{l}5 \\
5 \\
5 \\
5\end{array}$ & $\begin{array}{l}5 \\
5 \\
5 \\
5\end{array}$ \\
\hline & $\begin{array}{c}\text { epoxy } 1 \\
\text { epoxy } 2 \\
\text { polyurethane } \\
\text { acrylic }\end{array}$ & 35 & $\begin{array}{l}5 \\
5 \\
5 \\
3\end{array}$ & $\begin{array}{l}5 \\
5 \\
5 \\
5\end{array}$ & $\begin{array}{l}5 \\
5 \\
5 \\
2\end{array}$ & $\begin{array}{l}5 \\
5 \\
5 \\
5\end{array}$ & $\begin{array}{l}5 \\
5 \\
5 \\
5\end{array}$ & $\begin{array}{l}5 \\
5 \\
5 \\
5\end{array}$ \\
\hline & $\begin{array}{c}\text { epoxy } 1 \\
\text { epoxy } 2 \\
\text { polyurethane } \\
\text { acrylic }\end{array}$ & 49 & $\begin{array}{l}5 \\
5 \\
4 \\
3\end{array}$ & $\begin{array}{c}5 \\
5 \\
5 \\
4-5\end{array}$ & $\begin{array}{l}5 \\
5 \\
5 \\
2\end{array}$ & $\begin{array}{l}5 \\
5 \\
5 \\
5\end{array}$ & $\begin{array}{l}5 \\
5 \\
5 \\
5\end{array}$ & $\begin{array}{l}5 \\
5 \\
5 \\
5\end{array}$ \\
\hline & $\begin{array}{c}\text { epoxy } 1 \\
\text { epoxy } 2 \\
\text { polyurethane } \\
\text { acrylic }\end{array}$ & 63 & $\begin{array}{l}5 \\
5 \\
5 \\
3\end{array}$ & $\begin{array}{l}5 \\
5 \\
5 \\
4-5\end{array}$ & $\begin{array}{l}5 \\
5 \\
5 \\
2\end{array}$ & $\begin{array}{l}5 \\
5 \\
5 \\
5\end{array}$ & $\begin{array}{l}5 \\
5 \\
5 \\
5\end{array}$ & $\begin{array}{l}5 \\
5 \\
5 \\
5\end{array}$ \\
\hline \multirow[t]{5}{*}{$\begin{array}{l}\text { Mil. Spec. } \\
\text { PUb } \\
\text { Painted } \\
\text { Steel }\end{array}$} & $\begin{array}{c}\text { epoxy } 1 \\
\text { epoxy } 2 \\
\text { polyurethane } \\
\text { acrylic }\end{array}$ & 7 & $\begin{array}{c}5 \\
5 \\
4-5 \\
4\end{array}$ & $\begin{array}{l}5 \\
5 \\
5 \\
5\end{array}$ & $\begin{array}{l}5 \\
5 \\
5 \\
5\end{array}$ & $\begin{array}{l}5 \\
5 \\
5 \\
5\end{array}$ & $\begin{array}{l}5 \\
5 \\
5 \\
5\end{array}$ & $\begin{array}{l}5 \\
5 \\
5 \\
5\end{array}$ \\
\hline & $\begin{array}{c}\text { epoxy } 1 \\
\text { epoxy } 2 \\
\text { polyurethane } \\
\text { acrylic }\end{array}$ & 21 & $\begin{array}{c}5 \\
5 \\
4-5 \\
4\end{array}$ & $\begin{array}{l}5 \\
5 \\
5 \\
5\end{array}$ & $\begin{array}{l}5 \\
5 \\
5 \\
5\end{array}$ & $\begin{array}{l}5 \\
5 \\
5 \\
5\end{array}$ & $\begin{array}{l}5 \\
5 \\
5 \\
5\end{array}$ & $\begin{array}{l}5 \\
5 \\
5 \\
5\end{array}$ \\
\hline & $\begin{array}{c}\text { epoxy } 1 \\
\text { epoxy } 2 \\
\text { polyurethane } \\
\text { acrylic }\end{array}$ & 35 & $\begin{array}{c}5 \\
5 \\
4-5 \\
4\end{array}$ & $\begin{array}{l}5 \\
5 \\
5 \\
5\end{array}$ & $\begin{array}{l}5 \\
5 \\
5 \\
5\end{array}$ & $\begin{array}{l}5 \\
5 \\
5 \\
5\end{array}$ & $\begin{array}{l}5 \\
5 \\
5 \\
5\end{array}$ & $\begin{array}{l}5 \\
5 \\
5 \\
5\end{array}$ \\
\hline & $\begin{array}{c}\text { epoxy } 1 \\
\text { epoxy } 2 \\
\text { polyurethane } \\
\text { acrylic }\end{array}$ & 49 & $\begin{array}{c}5 \\
5 \\
4-5 \\
4\end{array}$ & $\begin{array}{l}5 \\
5 \\
5 \\
5\end{array}$ & $\begin{array}{l}5 \\
5 \\
5 \\
5\end{array}$ & $\begin{array}{l}5 \\
5 \\
5 \\
5\end{array}$ & $\begin{array}{l}5 \\
5 \\
5 \\
5\end{array}$ & $\begin{array}{l}5 \\
5 \\
5 \\
5\end{array}$ \\
\hline & $\begin{array}{c}\text { epoxy } 1 \\
\text { epoxy } 2 \\
\text { polyurethane } \\
\text { acrylic }\end{array}$ & 63 & $\begin{array}{c}5 \\
5 \\
4-5 \\
4\end{array}$ & $\begin{array}{l}5 \\
5 \\
5 \\
5\end{array}$ & $\begin{array}{l}5 \\
5 \\
5 \\
5\end{array}$ & $\begin{array}{l}5 \\
5 \\
5 \\
5\end{array}$ & $\begin{array}{l}5 \\
5 \\
5 \\
5\end{array}$ & $\begin{array}{l}5 \\
5 \\
5 \\
5\end{array}$ \\
\hline
\end{tabular}

a See Table 3.8 for a description of the numerical ranking code for assessing tamper tapes.

b Polyurethanc 
Table 4.5. Evaluation of Tamper Tapes Prepared from Different Confirm ${ }^{\circledR}$ Materials, Applied with Candidate Rapid-Set Adhesives, and Exposed in Daytona Beach, Florida

\begin{tabular}{|c|c|c|c|c|c|c|c|c|c|}
\hline \multirow[b]{3}{*}{ Surface } & \multirow[b]{3}{*}{$\begin{array}{c}\text { Confirm } \\
\text { Type }\end{array}$} & \multirow[b]{3}{*}{ Adhesive } & \multirow[b]{3}{*}{$\begin{array}{c}\text { Months } \\
\text { Exposure }\end{array}$} & \multicolumn{6}{|c|}{ Condition of Tamper Tape ${ }^{a}$} \\
\hline & & & & \multicolumn{2}{|c|}{ Appearance } & \multicolumn{2}{|c|}{ Security Emblem } & \multicolumn{2}{|c|}{ Adhesion to Surface } \\
\hline & & & & $\begin{array}{c}\text { Window } \\
\text { Area } \\
\end{array}$ & Vinyl Area & $\begin{array}{c}\text { Window } \\
\text { Area }\end{array}$ & Vinyl Area & $\begin{array}{c}\text { Window } \\
\text { Area }\end{array}$ & Overall \\
\hline \multirow[t]{3}{*}{ Aluminum } & $\begin{array}{c}1500 \\
\text { w/o primer }\end{array}$ & $\begin{array}{c}\text { epoxy } 1 \\
\text { expoxy } 2 \\
\text { polyurethane } \\
\text { acrylic }\end{array}$ & 0 & $\begin{array}{c}5 \\
5 \\
4-5 \\
2\end{array}$ & $\begin{array}{l}5 \\
5 \\
5 \\
2\end{array}$ & $\begin{array}{l}5 \\
5 \\
5 \\
3\end{array}$ & $\begin{array}{l}5 \\
5 \\
5 \\
3\end{array}$ & $\begin{array}{l}5 \\
5 \\
5 \\
5\end{array}$ & $\begin{array}{l}5 \\
5 \\
5 \\
5\end{array}$ \\
\hline & & $\begin{array}{c}\text { epoxy } 1 \\
\text { expoxy } 2 \\
\text { polyurethane } \\
\text { acrylic }\end{array}$ & 1 & $\begin{array}{c}4-5 \\
4-5 \\
4 \\
2\end{array}$ & $\begin{array}{c}4 \\
4-5 \\
5 \\
2\end{array}$ & $\begin{array}{l}\text { b } \\
- \\
- \\
-\end{array}$ & $\begin{array}{l}- \\
- \\
-\end{array}$ & $\begin{array}{l}5 \\
5 \\
5 \\
5\end{array}$ & $\begin{array}{l}5 \\
5 \\
5 \\
5\end{array}$ \\
\hline & & $\begin{array}{c}\text { epoxy } 1 \\
\text { expoxy } 2 \\
\text { polyurethane } \\
\text { acrylic }\end{array}$ & 2 & $\begin{array}{c}4-5 \\
4-5 \\
4 \\
2\end{array}$ & $\begin{array}{c}4-5 \\
4-5 \\
5 \\
2\end{array}$ & $\begin{array}{l}3 c \\
4 c \\
3 c \\
1\end{array}$ & $\begin{array}{l}3^{c} \\
4^{c} \\
4^{c} \\
2\end{array}$ & $\begin{array}{l}5 \\
5 \\
5 \\
5\end{array}$ & $\begin{array}{l}5 \\
5 \\
5 \\
5\end{array}$ \\
\hline \multirow[t]{3}{*}{ Steel } & $\begin{array}{c}1500 \\
\text { w/o primer }\end{array}$ & $\begin{array}{c}\text { epoxy } 1 \\
\text { expoxy } 2 \\
\text { polyurethane } \\
\text { acrylic }\end{array}$ & 0 & $\begin{array}{c}4-5 \\
4-5 \\
5 \\
2\end{array}$ & $\begin{array}{l}5 \\
5 \\
5 \\
2\end{array}$ & $\begin{array}{l}5 \\
5 \\
5 \\
3\end{array}$ & $\begin{array}{l}5 \\
5 \\
5 \\
3\end{array}$ & $\begin{array}{l}5 \\
5 \\
5 \\
5\end{array}$ & $\begin{array}{l}5 \\
5 \\
5 \\
5\end{array}$ \\
\hline & & $\begin{array}{c}\text { epoxy } 1 \\
\text { expoxy } 2 \\
\text { polyurethane } \\
\text { acrylic }\end{array}$ & 1 & $\begin{array}{c}4-5 \\
4-5 \\
4 \\
2\end{array}$ & $\begin{array}{c}4 \\
4-5 \\
5 \\
2\end{array}$ & - & $\begin{array}{l}- \\
- \\
-\end{array}$ & $\begin{array}{l}5 \\
5 \\
5 \\
5\end{array}$ & $\begin{array}{l}5 \\
5 \\
5 \\
5\end{array}$ \\
\hline & & $\begin{array}{c}\text { epoxy } 1 \\
\text { expoxy } 2 \\
\text { polyurethane } \\
\text { acrylic } \\
\end{array}$ & 2 & $\begin{array}{c}4-5 \\
4-5 \\
4 \\
2 \\
\end{array}$ & $\begin{array}{c}4 \\
4-5 \\
5 \\
2 \\
\end{array}$ & $\begin{array}{l}4 \\
5 \\
5 \\
1 \\
\end{array}$ & $\begin{array}{l}4 \\
5 \\
5 \\
2\end{array}$ & $\begin{array}{l}5 \\
5 \\
5 \\
5\end{array}$ & $\begin{array}{r}5 \\
5 \\
5 \\
5\end{array}$ \\
\hline
\end{tabular}

a See Table 3.8 for a description of the numerical ranking code for assessing tamper tapes.

b No data

c Emblem was black. 
Table 4.5. (Continued)

\begin{tabular}{|c|c|c|c|c|c|c|c|c|c|}
\hline \multirow[b]{3}{*}{ Surface } & \multirow[b]{3}{*}{$\begin{array}{c}\text { Confirm } \\
\text { Type }\end{array}$} & \multirow[b]{3}{*}{ Adhesive } & \multirow[b]{3}{*}{$\begin{array}{c}\text { Months } \\
\text { Exposure }\end{array}$} & \multicolumn{6}{|c|}{ Condition of Tamper Tape ${ }^{a}$} \\
\hline & & & & \multicolumn{2}{|c|}{ Appearance } & \multicolumn{2}{|c|}{ Security Emblem } & \multicolumn{2}{|c|}{ Adhesion to Surface } \\
\hline & & & & $\begin{array}{c}\text { Window } \\
\text { Area }\end{array}$ & Vinyl Area & $\begin{array}{c}\text { Window } \\
\text { Area }\end{array}$ & Vinyl Area & $\begin{array}{c}\text { Window } \\
\text { Area }\end{array}$ & Overal \\
\hline \multirow[t]{3}{*}{$\begin{array}{l}\text { Polyester } \\
\text { Fiberglass } \\
\text { Board }\end{array}$} & $\begin{array}{c}1500 \\
\text { w/o primer }\end{array}$ & $\begin{array}{c}\text { epoxy } 1 \\
\text { epoxy } 2 \\
\text { polyurethane } \\
\text { acrlyic }\end{array}$ & 0 & $\begin{array}{l}5 \\
5 \\
5 \\
2\end{array}$ & $\begin{array}{l}5 \\
5 \\
5 \\
3\end{array}$ & $\begin{array}{l}5 \\
5 \\
5 \\
2\end{array}$ & $\begin{array}{l}5 \\
5 \\
5 \\
3\end{array}$ & $\begin{array}{l}5 \\
5 \\
5 \\
5\end{array}$ & $\begin{array}{l}5 \\
5 \\
5 \\
5\end{array}$ \\
\hline & - & $\begin{array}{c}\text { epoxy } 1 \\
\text { epoxy } 2 \\
\text { polyurethane } \\
\text { acrlyic }\end{array}$ & 1 & $\begin{array}{c}4-5 \\
5 \\
4-5 \\
2\end{array}$ & $\begin{array}{c}4 \\
4-5 \\
5 \\
3\end{array}$ & $\begin{array}{l}-b \\
- \\
- \\
-\end{array}$ & $\begin{array}{l}- \\
- \\
-\end{array}$ & $\begin{array}{l}5 \\
5 \\
5 \\
5\end{array}$ & $\begin{array}{l}5 \\
5 \\
5 \\
5\end{array}$ \\
\hline & & $\begin{array}{c}\text { epoxy } 1 \\
\text { epoxy } 2 \\
\text { polyurethane } \\
\text { acriyic }\end{array}$ & 2 & $\begin{array}{c}4-5 \\
5 \\
4 \\
2\end{array}$ & $\begin{array}{c}4 \\
4-5 \\
5 \\
3\end{array}$ & $\begin{array}{c}2^{c} \\
4^{c} \\
3-4^{c} \\
3^{c}\end{array}$ & $\begin{array}{c}2^{c} \\
4^{c} \\
3-4^{c} \\
3^{c}\end{array}$ & $\begin{array}{l}5 \\
5 \\
5 \\
5\end{array}$ & $\begin{array}{l}5 \\
5 \\
5 \\
5\end{array}$ \\
\hline \multirow[t]{3}{*}{$\begin{array}{l}\text { Mil. Spec. } \\
\text { Polyurethane } \\
\text { Painted Steel } \\
\text { Panel }\end{array}$} & $\begin{array}{c}1500 \\
\text { w/o primer }\end{array}$ & $\begin{array}{c}\text { epoxy } 1 \\
\text { cpoxy } 2 \\
\text { polyurethane } \\
\text { acrlyic }\end{array}$ & 0 & $\begin{array}{c}5 \\
5 \\
4-5 \\
2\end{array}$ & $\begin{array}{l}5 \\
5 \\
5 \\
3\end{array}$ & $\begin{array}{l}5 \\
5 \\
5 \\
2\end{array}$ & $\begin{array}{l}5 \\
5 \\
5 \\
3\end{array}$ & $\begin{array}{l}5 \\
5 \\
5 \\
5\end{array}$ & $\begin{array}{l}5 \\
5 \\
5 \\
5\end{array}$ \\
\hline & & $\begin{array}{c}\text { epoxy } 1 \\
\text { epoxy } 2 \\
\text { polyurethane } \\
\text { acrlyic }\end{array}$ & 1 & $\begin{array}{l}5 \\
5 \\
4 \\
2\end{array}$ & $\begin{array}{c}4 \\
4-5 \\
5 \\
3\end{array}$ & $\begin{array}{l}- \\
- \\
-\end{array}$ & $\begin{array}{l}- \\
- \\
-\end{array}$ & $\begin{array}{l}5 \\
5 \\
5 \\
5\end{array}$ & $\begin{array}{l}5 \\
5 \\
5 \\
5\end{array}$ \\
\hline & & $\begin{array}{c}\text { epoxy } 1 \\
\text { epoxy } 2 \\
\text { polyurethane } \\
\text { acrlyic }\end{array}$ & 2 & $\begin{array}{l}5 \\
5 \\
4 \\
2\end{array}$ & $\begin{array}{c}4 \\
4-5 \\
5 \\
3\end{array}$ & $\begin{array}{l}2^{c} \\
5 \\
4^{c} \\
2^{c}\end{array}$ & $\begin{array}{c}2^{c} \\
3-4^{c} \\
3^{c} \\
2^{c}\end{array}$ & $\begin{array}{l}5 \\
5 \\
5 \\
5\end{array}$ & $\begin{array}{l}5 \\
5 \\
5 \\
5\end{array}$ \\
\hline
\end{tabular}

a See Table 3.8 for a description of the numerical ranking code for assessing tamper tapes.

b No data

c Emblem was black. 
Table 4.5. (Continued)

\begin{tabular}{|c|c|c|c|c|c|c|c|c|c|}
\hline \multirow[b]{3}{*}{ Surface } & \multirow[b]{3}{*}{$\begin{array}{c}\text { Confirm } \\
\text { Type }\end{array}$} & \multirow[b]{3}{*}{ Adhesive } & \multirow[b]{3}{*}{$\begin{array}{l}\text { Months } \\
\text { Exposure }\end{array}$} & \multicolumn{6}{|c|}{ Condition of Tamper Tape ${ }^{a}$} \\
\hline & & & & \multicolumn{2}{|c|}{ Appearance } & \multicolumn{2}{|c|}{ Security Emblem } & \multicolumn{2}{|c|}{ Adhesion to Surface } \\
\hline & & & & $\begin{array}{c}\text { Window } \\
\text { Area }\end{array}$ & Vinyl Area & $\begin{array}{c}\text { Window } \\
\text { Area }\end{array}$ & Vinyl Area & $\begin{array}{c}\text { Window } \\
\text { Area }\end{array}$ & Overall \\
\hline \multirow[t]{3}{*}{ Aluminum } & $\begin{array}{c}1500 \\
\text { w/primer }\end{array}$ & $\begin{array}{c}\text { epoxy } 1 \\
\text { epoxy } 2 \\
\text { polyurethane } \\
\text { acrylic }\end{array}$ & 0 & $\begin{array}{c}5 \\
4-5 \\
4-5 \\
4\end{array}$ & $\begin{array}{l}5 \\
5 \\
5 \\
5\end{array}$ & $\begin{array}{l}5 \\
5 \\
5 \\
5\end{array}$ & $\begin{array}{l}5 \\
.5 \\
.5 \\
5\end{array}$ & $\begin{array}{l}5 \\
5 \\
5 \\
5\end{array}$ & $\begin{array}{l}5 \\
5 \\
5 \\
5\end{array}$ \\
\hline & & $\begin{array}{c}\text { epoxy } 1 \\
\text { epoxy } 2 \\
\text { polyurethane } \\
\text { acrylic }\end{array}$ & 1 & $\begin{array}{c}4-5 \\
4-5 \\
4 \\
4\end{array}$ & $\begin{array}{c}4 \\
5 \\
5 \\
4-5\end{array}$ & $\begin{array}{l}-b \\
- \\
- \\
-\end{array}$ & $\begin{array}{l}- \\
- \\
-\end{array}$ & $\begin{array}{l}5 \\
5 \\
5 \\
5\end{array}$ & $\begin{array}{l}5 \\
5 \\
5 \\
5\end{array}$ \\
\hline & & $\begin{array}{l}\text { epoxy } 1 \\
\text { epoxy } 2 \\
\text { polyurethane } \\
\text { acrylic }\end{array}$ & 2 & $\begin{array}{c}4-5 \\
4-5 \\
4 \\
4\end{array}$ & $\begin{array}{l}4 \\
5 \\
5 \\
5\end{array}$ & $\begin{array}{c}2^{c} \\
4 c \\
4-5 \\
1\end{array}$ & $\begin{array}{l}2^{c} \\
4^{c} \\
4-5 \\
2\end{array}$ & $\begin{array}{l}5 \\
5 \\
5 \\
5\end{array}$ & $\begin{array}{l}5 \\
5 \\
5 \\
5\end{array}$ \\
\hline \multirow[t]{3}{*}{ Steel } & $\begin{array}{c}1500 \\
\text { w/primer }\end{array}$ & $\begin{array}{c}\text { epoxy } 1 \\
\text { epoxy } 2 \\
\text { polyurethane } \\
\text { acrylic }\end{array}$ & 0 & $\begin{array}{c}5 \\
5 \\
4-5 \\
4\end{array}$ & $\begin{array}{l}5 \\
5 \\
5 \\
4\end{array}$ & $\begin{array}{l}5 \\
5 \\
5 \\
5\end{array}$ & $\begin{array}{l}5 \\
5 \\
5 \\
5\end{array}$ & $\begin{array}{l}5 \\
5 \\
5 \\
5\end{array}$ & $\begin{array}{l}5 \\
5 \\
5 \\
5\end{array}$ \\
\hline & & $\begin{array}{c}\text { epoxy } 1 \\
\text { epoxy } 2 \\
\text { polyurethane } \\
\text { acrylic }\end{array}$ & 1 & $\begin{array}{c}5 \\
5 \\
4-5 \\
4-5\end{array}$ & $\begin{array}{c}4 \\
5 \\
4-5 \\
4-5\end{array}$ & $\begin{array}{l}- \\
- \\
-\end{array}$ & $\begin{array}{l}- \\
- \\
-\end{array}$ & $\begin{array}{l}5 \\
5 \\
5 \\
5\end{array}$ & $\begin{array}{l}5 \\
5 \\
5 \\
5\end{array}$ \\
\hline & & $\begin{array}{c}\text { epoxy } 1 \\
\text { epoxy } 2 \\
\text { polyurethane } \\
\text { acrylic }\end{array}$ & 2 & $\begin{array}{c}5 \\
5 \\
4-5 \\
4-5\end{array}$ & $\begin{array}{c}4 \\
5 \\
4-5 \\
4-5\end{array}$ & $\begin{array}{c}3 \\
3^{c} \\
5 \\
2\end{array}$ & $\begin{array}{l}3-4 \\
3^{c} \\
5 \\
2\end{array}$ & $\begin{array}{r}5 \\
5 \\
-\quad 5 \\
5\end{array}$ & $\begin{array}{l}5 \\
5 \\
5 \\
5\end{array}$ \\
\hline
\end{tabular}

\footnotetext{
a See Table 3.8 for a description of the numerical ranking code for assessing tamper tapes.

b No data

c Emblem was black.
} 
Table 4.5. (Continued)

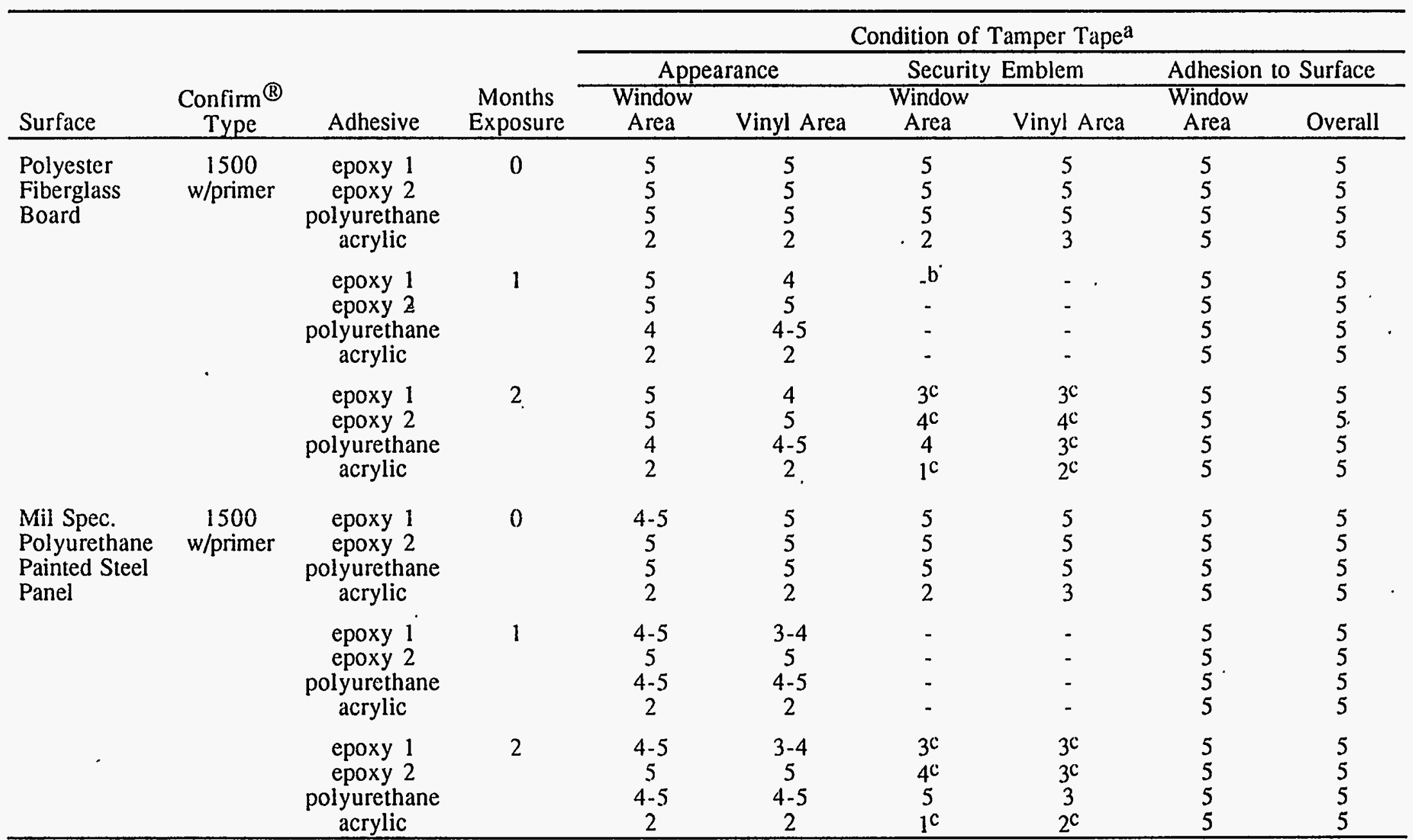

a See Table 3.8 for a description of the numerical ranking code for assessing tamper tapes.

b No data

c Emblem was black. 
Table 4.5. (Continued)

\begin{tabular}{|c|c|c|c|c|c|c|c|c|c|}
\hline \multirow[b]{3}{*}{ Surface } & \multirow[b]{3}{*}{$\begin{array}{c}\text { Confirm }^{\circledR} \\
\text { Type }\end{array}$} & \multirow[b]{3}{*}{ Adhesive } & \multirow[b]{3}{*}{$\begin{array}{c}\text { Months } \\
\text { Exposure }\end{array}$} & \multicolumn{6}{|c|}{ Condition of Tamper Tape } \\
\hline & & & & \multicolumn{2}{|c|}{ Appearance } & \multicolumn{2}{|c|}{ Security Emblem } & \multicolumn{2}{|c|}{ Adhesion to Surface } \\
\hline & & & & $\begin{array}{c}\text { Window } \\
\text { Area }\end{array}$ & Vinyl Area & $\begin{array}{c}\text { Window } \\
\text { Area }\end{array}$ & Vinyl Area & $\begin{array}{c}\text { Window } \\
\text { Area }\end{array}$ & Overall \\
\hline \multirow[t]{3}{*}{ Aluminum } & 1300 & $\begin{array}{c}\text { epoxy } 1 \\
\text { epoxy } 2 \\
\text { polyurethane } \\
\text { acrylic }\end{array}$ & 0 & $\begin{array}{l}5 \\
4-5 \\
4-5 \\
4-5\end{array}$ & $\begin{array}{l}5 \\
5 \\
5 \\
5\end{array}$ & $\begin{array}{l}5 \\
5 \\
5 \\
5\end{array}$ & $\begin{array}{l}5 \\
5 \\
5 \\
5\end{array}$ & $\begin{array}{l}5 \\
5 \\
5 \\
5\end{array}$ & $\begin{array}{l}5 \\
5 \\
5 \\
5\end{array}$ \\
\hline & & $\begin{array}{c}\text { epoxy } 1 \\
\text { epoxy } 2 \\
\text { polyurethane } \\
\text { acrylic }\end{array}$ & 11 & $\begin{array}{c}4 \\
4-5 \\
3-4 \\
4\end{array}$ & $\begin{array}{l}4 \\
5 \\
5 \\
5\end{array}$ & $\begin{array}{l}-b \\
- \\
- \\
-\end{array}$ & $\begin{array}{l}- \\
- \\
-\end{array}$ & $\begin{array}{l}5 \\
5 \\
5 \\
5\end{array}$ & $\begin{array}{l}5 \\
5 \\
5 \\
5\end{array}$ \\
\hline & & $\begin{array}{c}\text { epoxy } 1 \\
\text { epoxy } 2 \\
\text { polyurethane } \\
\text { acrylic }\end{array}$ & 2 & $\begin{array}{c}4 \\
4-5 \\
3-4 \\
4\end{array}$ & $\begin{array}{l}4 \\
5 \\
5 \\
5\end{array}$ & $\begin{array}{l}5 \\
5 \\
1 \\
4\end{array}$ & $\begin{array}{l}4 \\
5 \\
2 \\
4\end{array}$ & $\begin{array}{l}5 \\
5 \\
5 \\
5\end{array}$ & $\begin{array}{l}5 \\
5 \\
5 \\
5\end{array}$ \\
\hline \multirow[t]{3}{*}{ Steel } & 1300 & $\begin{array}{c}\text { epoxy } 1 \\
\text { epoxy } 2 \\
\text { polyurethane } \\
\text { acrylic }\end{array}$ & 0 & $\begin{array}{l}5 \\
4 \\
5 \\
5\end{array}$ & $\begin{array}{l}5 \\
5 \\
5 \\
5\end{array}$ & $\begin{array}{l}5 \\
5 \\
5 \\
5\end{array}$ & $\begin{array}{l}5 \\
5 \\
5 \\
5\end{array}$ & $\begin{array}{l}5 \\
5 \\
5 \\
5\end{array}$ & $\begin{array}{l}5 \\
5 \\
5 \\
5\end{array}$ \\
\hline & & $\begin{array}{l}\text { epoxy } 1 \\
\text { epoxy } 2 \\
\text { polyurethane } \\
\text { acrylic }\end{array}$ & 1 & $\begin{array}{c}5 \\
4 \\
4 \\
4-5\end{array}$ & $\begin{array}{l}4 \\
5 \\
5 \\
5\end{array}$ & $\begin{array}{l}- \\
- \\
-\end{array}$ & $\begin{array}{l}-- \\
- \\
-\end{array}$ & $\begin{array}{l}5 \\
5 \\
5 \\
5\end{array}$ & $\begin{array}{l}5 \\
5 \\
5 \\
5\end{array}$ \\
\hline & & $\begin{array}{c}\text { epoxy } 1 \\
\text { epoxy } 2 \\
\text { polyurethane } \\
\text { acrylic }\end{array}$ & 2 & $\begin{array}{c}5 \\
4 \\
4 \\
4-5\end{array}$ & $\begin{array}{l}4 \\
5 \\
5 \\
5\end{array}$ & $\begin{array}{l}4 \\
5 \\
1 \\
4\end{array}$ & $\begin{array}{c}5 \\
5 \\
2 \\
2-3\end{array}$ & $\begin{array}{l}5 \\
5 \\
5 \\
5\end{array}$ & $\begin{array}{l}5 \\
5 \\
5 \\
5\end{array}$ \\
\hline
\end{tabular}

a See Table 3.8 for a description of the numerical ranking code for assessing tamper tapes.

b No data 
Table 4.5. (Continued)

\begin{tabular}{|c|c|c|c|c|c|c|c|c|c|}
\hline \multirow[b]{3}{*}{ Surface } & \multirow[b]{3}{*}{$\begin{array}{c}\text { Confirm }^{\circledR} \\
\text { Type }\end{array}$} & \multirow[b]{3}{*}{ Adhesive } & \multirow[b]{3}{*}{$\begin{array}{c}\text { Months } \\
\text { Exposure }\end{array}$} & \multicolumn{6}{|c|}{ Condition of Tamper Tape ${ }^{\mathrm{a}}$} \\
\hline & & & & \multicolumn{2}{|c|}{ Appearance } & \multicolumn{2}{|c|}{ Security Emblem } & \multicolumn{2}{|c|}{ Adhesion to Surface } \\
\hline & & & & $\begin{array}{c}\text { Window } \\
\text { Area }\end{array}$ & Vinyl Area & $\begin{array}{c}\text { Window } \\
\text { Area }\end{array}$ & Vinyl Area & $\begin{array}{c}\text { Window } \\
\text { Area }\end{array}$ & Overall \\
\hline \multirow[t]{3}{*}{$\begin{array}{l}\text { Polyester } \\
\text { Fiberglass } \\
\text { Board }\end{array}$} & 1300 & $\begin{array}{c}\text { epoxy } 1 \\
\text { epoxy } 2 \\
\text { polyurethane } \\
\text { acrylic }\end{array}$ & 0. & $\begin{array}{c}5 \\
4-5 \\
5 \\
4\end{array}$ & $\begin{array}{l}5 \\
5 \\
5 \\
5\end{array}$ & $\begin{array}{l}5 \\
5 \\
5 \\
5\end{array}$ & $\begin{array}{l}5 \\
5 \\
5 \\
5\end{array}$ & $\begin{array}{l}5 \\
5 \\
5 \\
5\end{array}$ & $\begin{array}{l}5 \\
5 \\
5 \\
5\end{array}$ \\
\hline & & $\begin{array}{l}\text { epoxy } 1 \\
\text { epoxy } 2 \\
\text { polyurethane } \\
\text { acrylic }\end{array}$ & 1 & $\begin{array}{c}5 \\
4-5 \\
4-5 \\
4\end{array}$ & $\begin{array}{c}4-5 \\
5 \\
5 \\
5\end{array}$ & $\begin{array}{l}-b \\
- \\
- \\
-\end{array}$ & $\begin{array}{l}- \\
- \\
-\end{array}$ & $\begin{array}{l}5 \\
5 \\
5 \\
5\end{array}$ & $\begin{array}{l}5 \\
5 \\
5 \\
5\end{array}$ \\
\hline & & $\begin{array}{c}\text { epoxy } 1 \\
\text { epoxy } 2 \\
\text { polyurethane } \\
\text { acrylic }\end{array}$ & 2 & $\begin{array}{c}4 \\
4-5 \\
4-5 \\
4\end{array}$ & $\begin{array}{c}4-5 \\
5 \\
5 \\
5\end{array}$ & $\begin{array}{c}5 \\
5 \\
1 \\
4^{c}\end{array}$ & $\begin{array}{c}5 \\
5 \\
3 \\
4^{c}\end{array}$ & $\begin{array}{l}5 \\
5 \\
5 \\
5\end{array}$ & $\begin{array}{l}5 \\
5 \\
5 \\
5\end{array}$ \\
\hline \multirow[t]{3}{*}{$\begin{array}{l}\text { Mil Spec. } \\
\text { Polyurethane } \\
\text { Painted Steel } \\
\text { Panel }\end{array}$} & 1300 & $\begin{array}{l}\text { epoxy } 1 \\
\text { epoxy } 2 \\
\text { polyurethane } \\
\text { acrylic }\end{array}$ & 0 & $\begin{array}{c}5 \\
5 \\
5 \\
4-5\end{array}$ & $\begin{array}{l}5 \\
5 \\
5 \\
5\end{array}$ & $\begin{array}{l}5 \\
5 \\
5 \\
5\end{array}$ & $\begin{array}{l}5 \\
5 \\
5 \\
5\end{array}$ & $\begin{array}{l}5 \\
5 \\
5 \\
5\end{array}$ & $\begin{array}{l}5 \\
5 \\
5 \\
5\end{array}$ \\
\hline & & $\begin{array}{c}\text { epoxy } 1 \\
\text { epoxy } 2 \\
\text { polyurethane } \\
\text { acrylic }\end{array}$ & 1 & $\begin{array}{c}5 \\
5 \\
4 \\
4-5\end{array}$ & $\begin{array}{l}4 \\
5 \\
5 \\
5\end{array}$ & $\begin{array}{l}- \\
- \\
-\end{array}$ & $\begin{array}{l}- \\
- \\
-\end{array}$ & $\begin{array}{l}5 \\
5 \\
5 \\
5\end{array}$ & $\begin{array}{l}5 \\
5 \\
5 \\
5\end{array}$ \\
\hline & & $\begin{array}{c}\text { epoxy } 1 \\
\text { epoxy } 2 \\
\text { polyurethane } \\
\text { acrylic }\end{array}$ & 2 & $\begin{array}{c}5 \\
5 \\
4 \\
4-5\end{array}$ & $\begin{array}{c}4 \\
5 \\
5 \\
4-5\end{array}$ & $\begin{array}{c}3 c \\
5 \\
1-2 \\
3\end{array}$ & $\begin{array}{l}3 c \\
4 \\
3 \\
3 \\
\end{array}$ & $\begin{array}{l}5 \\
5 \\
5 \\
5\end{array}$ & $\begin{array}{l}5 \\
5 \\
5 \\
5\end{array}$ \\
\hline
\end{tabular}

\footnotetext{
a See Table 3.8 for a description of the numerical ranking code for assessing tamper tapes.
}

b No data

c Emblem was black. 
beads of the Confirm ${ }^{\circledR}$ material. The change is attributed to the salt spray in the air since the tapes exposed in the QUV cabinet did not show this effect. Security features on only 2 out of 16 of the tamper tapes prepared from the latex Confirm $^{\circledR} 1300$ turned black. With the Confirm ${ }^{\circledR} 1500$ materials, however, there were 10 or 11 out of 16 that showed this effect.

Of the four rapid-set adhesives on all the tamper tapes, epoxy 2 showed the least amount of change in the visibility of the security features. Only the polyurethane adhesive performed better with tamper tapes prepared from the Confirm ${ }^{\circledR} 1500$ with primer. Tamper tapes prepared with the polyurethane adhesive and the latex Confirm ${ }^{\circledR}(1300)$ lost the visibility of their security features. Overall, the second best adhesive was epoxy 1 . However, with tamper tapes made with the Confirm ${ }^{\circledR} 1500$ products, the epoxy 1 did not do well on the fiberglass or the polyurethanepainted steel surfaces. With the exception of the polyurethane, the adhesives performed better on tamper tapes prepared with the latex Confirm ${ }^{\circledR}$ than on those using the Confirm ${ }^{\circledR} 1500$ materials.

\subsubsection{QUV Cabinet Exposure Results}

The results of the 42-day QUV cabinet exposure of tamper tapes made with Confirm ${ }^{\circledR} 1500$ without primer material are given in Table 4.6. The acrylic adhesive attacked this Confirm ${ }^{\circledR}$ material, as it did the 1700 material, causing poor appearance and poor visibility of the security feature. After 5 or 6 weeks, the security features were completely degraded. With the acrylic adhesive tamper tapes, there was also some loss of adhesion after 5 weeks. The visibility of the security features changed slightly on the epoxy 1 adhesive-bonded tamper tapes; moderate to full loss of visibility of the security feature occurred in the same amount of time with the polyurethane adhesive. The security features of the epoxy 2 adhesive-bonded tamper tapes did not show any loss of visibility, and the tapes prepared with this adhesive performed well overall.

Tamper tapes prepared with Confirm ${ }^{\circledR} 1500$ with primer material, bonded to the various surfaces with the rapid-set adhesives, and exposed in the QUV cabinet for 42 days performed very similarly to the Confirm ${ }^{\circledR} 1500$ without primer material (Table 4.7). Because of a shortage of the acrylic adhesive, another commercially-available acrylic adhesive was substituted when adhering the tamper tapes to steel. This acrylic adhesive did not attack or harm the security features, therefore, the tamper tapes aged better. This adhesive had a slower gel time compared to the acrylic adhesive used in all other weathering studies, but its gel time was still comparable to the epoxy 1 adhesive.

In general, the candidate adhesives performed better in the QUV cabinet on tamper tapes prepared with the Confirm ${ }^{\circledR} 1300$ material than they did on tamper tapes prepared with the other Confirm ${ }^{\circledR}$ materials (Table 4.8). The acrylic adhesive, which attacked all of the other Confirm ${ }^{\circledR}$ 
Table 4.6. Evaluation of Confirm ${ }^{\circledR} 1500$ Without Primer Tamper Tapes Applied with Candidate Rapid-Set Adhesives and Aged in QUV Cabinet (UV, $60^{\circ} \mathrm{C}$; Condensing Humidity, $\left.40^{\circ} \mathrm{C}\right)$

\begin{tabular}{|c|c|c|c|c|c|c|c|c|}
\hline \multirow[b]{3}{*}{ Surface } & \multirow[b]{3}{*}{ Adhesive } & \multirow[b]{3}{*}{$\begin{array}{c}\text { Days of } \\
\text { Exposure }\end{array}$} & \multicolumn{6}{|c|}{ Condition of Tamper Tape ${ }^{a}$} \\
\hline & & & \multicolumn{2}{|c|}{ Appearance } & \multicolumn{2}{|c|}{ Security Emblem } & \multicolumn{2}{|c|}{ Surface Adhesion } \\
\hline & & & $\begin{array}{c}\text { Window } \\
\text { Area }\end{array}$ & $\begin{array}{l}\text { Vinyl } \\
\text { Area }\end{array}$ & $\begin{array}{c}\text { Window } \\
\text { Area }\end{array}$ & $\begin{array}{l}\text { Vinyl } \\
\text { Area } \\
\end{array}$ & $\begin{array}{c}\text { Window } \\
\text { Area }\end{array}$ & Overall \\
\hline \multirow[t]{7}{*}{ Aluminum } & $\begin{array}{c}\text { epoxy } 1 \\
\text { epoxy } 2 \\
\text { polyurethane } \\
\text { acrylic }\end{array}$ & 0 & $\begin{array}{l}4-5 \\
4-5 \\
5 \\
4-5\end{array}$ & $\begin{array}{c}4-5 \\
4-5 \\
5 \\
4-5\end{array}$ & $\begin{array}{l}5 \\
5 \\
5 \\
3\end{array}$ & $\begin{array}{l}5 \\
5 \\
5 \\
3\end{array}$ & $\begin{array}{l}5 \\
5 \\
5 \\
5\end{array}$ & $\begin{array}{l}5 \\
5 \\
5 \\
5\end{array}$ \\
\hline & $\begin{array}{l}\text { epoxy } 1 \\
\text { epoxy } 2 \\
\text { polyurethane } \\
\text { acrylic }\end{array}$ & 7 & $\begin{array}{l}4 \\
4 \\
5 \\
2\end{array}$ & $\begin{array}{l}4 \\
4 \\
5 \\
2\end{array}$ & $\begin{array}{l}5 \\
5 \\
5 \\
2\end{array}$ & $\begin{array}{l}5 \\
5 \\
5 \\
2\end{array}$ & $\begin{array}{l}5 \\
5 \\
5 \\
5\end{array}$ & $\begin{array}{l}5 \\
5 \\
5 \\
5\end{array}$ \\
\hline & $\begin{array}{c}\text { epoxy } 1 \\
\text { epoxy } 2 \\
\text { polyurethane } \\
\text { acrylic }\end{array}$ & 14 & $\begin{array}{c}4 \\
4 \\
4-5 \\
2\end{array}$ & $\begin{array}{c}4 \\
4 \\
4-5 \\
2\end{array}$ & $\begin{array}{l}5 \\
5 \\
5 \\
2\end{array}$ & $\begin{array}{l}5 \\
5 \\
5 \\
2\end{array}$ & $\begin{array}{l}5 \\
5 \\
5 \\
5\end{array}$ & $\begin{array}{l}5 \\
5 \\
5 \\
5\end{array}$ \\
\hline & $\begin{array}{c}\text { epoxy } 1 \\
\text { epoxy } 2 \\
\text { polyurethane } \\
\text { acrylic }\end{array}$ & 21 & $\begin{array}{c}4 \\
4 \\
4-5 \\
2\end{array}$ & $\begin{array}{c}4 \\
4 \\
4-5 \\
2\end{array}$ & $\begin{array}{l}5 \\
5 \\
5 \\
2\end{array}$ & $\begin{array}{l}5 \\
5 \\
5 \\
2\end{array}$ & $\begin{array}{l}5 \\
5 \\
5 \\
5\end{array}$ & $\begin{array}{l}5 \\
5 \\
5 \\
5\end{array}$ \\
\hline & $\begin{array}{l}\text { cpoxy } 1 \\
\text { epoxy } 2 \\
\text { polyurethane } \\
\text { acrylic }\end{array}$ & 28 & $\begin{array}{c}4 \\
4 \\
4-5 \\
1\end{array}$ & $\begin{array}{c}4 \\
4 \\
4-5 \\
3\end{array}$ & $\begin{array}{l}4 \\
5 \\
5 \\
2\end{array}$ & $\begin{array}{l}5 \\
5 \\
4 \\
2\end{array}$ & $\begin{array}{l}5 \\
5 \\
5 \\
3\end{array}$ & $\begin{array}{l}5 \\
5 \\
5 \\
3\end{array}$ \\
\hline & $\begin{array}{c}\text { epoxy } 1 \\
\text { epoxy } 2 \\
\text { polyurethane } \\
\text { acrylic }\end{array}$ & 35 & $\begin{array}{l}4 \\
4 \\
4 \\
1\end{array}$ & $\begin{array}{c}3 \\
4 \\
4-5 \\
2\end{array}$ & $\begin{array}{l}4 \\
5 \\
4 \\
2\end{array}$ & $\begin{array}{l}4 \\
5 \\
4 \\
2\end{array}$ & $\begin{array}{l}5 \\
5 \\
5 \\
2\end{array}$ & $\begin{array}{l}5 \\
5 \\
5 \\
3\end{array}$ \\
\hline & $\begin{array}{l}\text { epoxy } 1 \\
\text { epoxy } 2 \\
\text { polyurethane } \\
\text { acrylic }\end{array}$ & 42 & $\begin{array}{l}3 \\
4 \\
3 \\
1\end{array}$ & $\begin{array}{c}3 \\
4 \\
4-5 \\
2\end{array}$ & $\begin{array}{l}4 \\
5 \\
4 \\
1\end{array}$ & $\begin{array}{l}4 \\
5 \\
3 \\
2\end{array}$ & $\begin{array}{l}5 \\
5 \\
5 \\
2\end{array}$ & $\begin{array}{l}5 \\
5 \\
5 \\
2\end{array}$ \\
\hline \multirow[t]{2}{*}{ Steel } & $\begin{array}{c}\text { cpoxy } 1 \\
\text { epoxy } 2 \\
\text { polyurethane } \\
\text { acrylic }\end{array}$ & 0 & $\begin{array}{c}4-5 \\
5 \\
4-5 \\
1\end{array}$ & $\begin{array}{c}4-5 \\
5 \\
4-5 \\
1\end{array}$ & $\begin{array}{l}5 \\
5 \\
5 \\
1\end{array}$ & $\begin{array}{l}5 \\
5 \\
5 \\
2\end{array}$ & $\begin{array}{l}5 \\
5 \\
5 \\
5\end{array}$ & $\begin{array}{l}5 \\
5 \\
5 \\
5\end{array}$ \\
\hline & $\begin{array}{c}\text { epoxy } 1 \\
\text { epoxy } 2 \\
\text { polyurethane } \\
\text { acrylic }\end{array}$ & 7 & $\begin{array}{c}4 \\
4 \\
4-5 \\
1\end{array}$ & $\begin{array}{c}4 \\
4 \\
4-5 \\
1\end{array}$ & $\begin{array}{l}5 \\
5 \\
5 \\
1\end{array}$ & $\begin{array}{l}5 \\
5 \\
5 \\
2\end{array}$ & $\begin{array}{l}5 \\
5 \\
5 \\
5\end{array}$ & $\begin{array}{r}5 \\
5 \\
5 \\
5\end{array}$ \\
\hline
\end{tabular}

a Sce Table 3.8 for a description of the numcrical ranking code for assessing tamper tapes. 
Table 4.6. (Continued)

\begin{tabular}{|c|c|c|c|c|c|c|c|c|}
\hline \multirow[b]{3}{*}{ Surface } & \multirow[b]{3}{*}{ Adhesive } & \multirow[b]{3}{*}{$\begin{array}{c}\text { Days of } \\
\text { Exposure }\end{array}$} & \multicolumn{6}{|c|}{ Condition of Tamper Tape ${ }^{a}$} \\
\hline & & & \multicolumn{2}{|c|}{ Appearance } & \multicolumn{2}{|c|}{ Security Emblem } & \multicolumn{2}{|c|}{ Surface Adhesion } \\
\hline & & & $\begin{array}{c}\text { Window } \\
\text { Area }\end{array}$ & $\begin{array}{l}\text { Vinyl } \\
\text { Area } \\
\end{array}$ & $\begin{array}{c}\text { Window } \\
\text { Area }\end{array}$ & $\begin{array}{l}\text { Vinyl } \\
\text { Area }\end{array}$ & $\begin{array}{c}\text { Window } \\
\text { Area }\end{array}$ & Overall \\
\hline \multirow[t]{5}{*}{$\begin{array}{l}\text { Steel } \\
\text { (Cont.) }\end{array}$} & $\begin{array}{c}\text { epoxy } 1 \\
\text { epoxy } 2 \\
\text { polyurethane } \\
\text { acrylic }\end{array}$ & 14 & $\begin{array}{c}3-4 \\
4 \\
4-5 \\
1\end{array}$ & $\begin{array}{c}3-4 \\
4 \\
4-5 \\
1\end{array}$ & $\begin{array}{l}5 \\
5 \\
5 \\
1\end{array}$ & $\begin{array}{l}5 \\
5 \\
5 \\
2\end{array}$ & $\begin{array}{l}5 \\
5 \\
5 \\
5\end{array}$ & $\begin{array}{l}5 \\
5 \\
5 \\
5\end{array}$ \\
\hline & $\begin{array}{c}\text { epoxy } 1 \\
\text { epoxy } 2 \\
\text { polyurethane } \\
\text { acrylic }\end{array}$ & 21 & $\begin{array}{c}3-4 \\
4 \\
4-5 \\
1\end{array}$ & $\begin{array}{c}3-4 \\
4 \\
4-5 \\
1\end{array}$ & $\begin{array}{l}5 \\
5 \\
5 \\
1\end{array}$ & $\begin{array}{l}5 \\
5 \\
5 \\
2\end{array}$ & $\begin{array}{l}5 \\
5 \\
5 \\
5\end{array}$ & $\begin{array}{l}5 \\
5 \\
5 \\
5\end{array}$ \\
\hline & $\begin{array}{l}\text { epoxy } 1 \\
\text { cpoxy } 2 \\
\text { polyurethane } \\
\text { acrylic }\end{array}$ & 28 & $\begin{array}{c}3-4 \\
4 \\
4 \\
1\end{array}$ & $\begin{array}{c}3-4 \\
4 \\
4-5 \\
1\end{array}$ & $\begin{array}{l}4 \\
5 \\
1 \\
1\end{array}$ & $\begin{array}{l}4 \\
5 \\
2 \\
2\end{array}$ & $\begin{array}{l}5 \\
5 \\
5 \\
3\end{array}$ & $\begin{array}{l}5 \\
5 \\
5 \\
4\end{array}$ \\
\hline & $\begin{array}{c}\text { epoxy } 1 \\
\text { epoxy } 2 \\
\text { polyurethane } \\
\text { acrylic }\end{array}$ & 35 & $\begin{array}{c}3-4 \\
4 \\
4 \\
1\end{array}$ & $\begin{array}{c}3-4 \\
4 \\
4-5 \\
1\end{array}$ & $\begin{array}{l}4 \\
5 \\
1 \\
1\end{array}$ & $\begin{array}{l}4 \\
5 \\
2 \\
2\end{array}$ & $\begin{array}{l}5 \\
5 \\
5 \\
3\end{array}$ & $\begin{array}{l}5 \\
5 \\
5 \\
4\end{array}$ \\
\hline & $\begin{array}{c}\text { epoxy } 1 \\
\text { epoxy } 2 \\
\text { polyurethane } \\
\text { acrylic }\end{array}$ & 42 & $\begin{array}{l}3 \\
4 \\
3 \\
1\end{array}$ & $\begin{array}{c}3 \\
4 \\
4-5 \\
1\end{array}$ & $\begin{array}{l}4 \\
5 \\
1 \\
1\end{array}$ & $\begin{array}{l}4 \\
5 \\
2 \\
2\end{array}$ & $\begin{array}{l}5 \\
5 \\
5 \\
3\end{array}$ & $\begin{array}{l}5 \\
5 \\
5 \\
3\end{array}$ \\
\hline \multirow[t]{5}{*}{$\begin{array}{l}\text { Polyester } \\
\text { Fiberglass } \\
\text { Board }\end{array}$} & $\begin{array}{c}\text { epoxy } 1 \\
\text { epoxy } 2 \\
\text { polyurcthane } \\
\text { acrylic }\end{array}$ & 0 & $\begin{array}{c}4-5 \\
5 \\
4-5 \\
3\end{array}$ & $\begin{array}{c}4-5 \\
5 \\
4-5 \\
4-5\end{array}$ & $\begin{array}{l}5 \\
5 \\
5 \\
5\end{array}$ & $\begin{array}{l}5 \\
5 \\
5 \\
5\end{array}$ & $\begin{array}{l}5 \\
5 \\
5 \\
5\end{array}$ & $\begin{array}{l}5 \\
5 \\
5 \\
5\end{array}$ \\
\hline & $\begin{array}{c}\text { epoxy } 1 \\
\text { epoxy } 2 \\
\text { polyurethane } \\
\text { acrylic }\end{array}$ & 7 & $\begin{array}{c}3-4 \\
4-5 \\
4-5 \\
2\end{array}$ & $\begin{array}{c}3-4 \\
4-5 \\
4-5 \\
4\end{array}$ & $\begin{array}{l}5 \\
5 \\
5 \\
3\end{array}$ & $\begin{array}{l}5 \\
5 \\
5 \\
3\end{array}$ & $\begin{array}{l}5 \\
5 \\
5 \\
5\end{array}$ & $\begin{array}{l}5 \\
5 \\
5 \\
5\end{array}$ \\
\hline & $\begin{array}{c}\text { epoxy } 1 \\
\text { cpoxy } 2 \\
\text { polyurcthanc } \\
\text { acrylic }\end{array}$ & 14 & $\begin{array}{l}3 \\
4 \\
4 \\
2\end{array}$ & $\begin{array}{l}3 \\
4 \\
4 \\
4\end{array}$ & $\begin{array}{l}5 \\
5 \\
2 \\
3\end{array}$ & $\begin{array}{l}5 \\
5 \\
5 \\
3\end{array}$ & $\begin{array}{l}5 \\
5 \\
5 \\
5\end{array}$ & $\begin{array}{l}5 \\
5 \\
5 \\
5\end{array}$ \\
\hline & $\begin{array}{c}\text { epoxy } 1 \\
\text { epoxy } 2 \\
\text { polyurethane } \\
\text { acrylic }\end{array}$ & 21 & $\begin{array}{c}3-4 \\
4 \\
4 \\
2\end{array}$ & $\begin{array}{c}3-4 \\
4 \\
4 \\
4\end{array}$ & $\begin{array}{l}5 \\
5 \\
2 \\
3\end{array}$ & $\begin{array}{l}5 \\
5 \\
5 \\
3\end{array}$ & $\begin{array}{l}5 \\
5 \\
5 \\
5\end{array}$ & $\begin{array}{l}5 \\
5 \\
5 \\
5\end{array}$ \\
\hline & $\begin{array}{c}\text { epoxy } 1 \\
\text { epoxy } 2 \\
\text { polyurethane } \\
\text { acrylic }\end{array}$ & 28 & $\begin{array}{l}3 \\
4 \\
3 \\
2\end{array}$ & $\begin{array}{l}3 \\
4 \\
4 \\
4\end{array}$ & $\begin{array}{l}4 \\
5 \\
2 \\
2\end{array}$ & $\begin{array}{l}4 \\
5 \\
3 \\
3\end{array}$ & $\begin{array}{l}5 \\
5 \\
5 \\
4\end{array}$ & $\begin{array}{l}5 \\
5 \\
5 \\
4\end{array}$ \\
\hline
\end{tabular}

a See Table 3.8 for a description of the numerical ranking code for assessing tamper tapes. 
Table 4.6. (Continued)

\begin{tabular}{|c|c|c|c|c|c|c|c|c|}
\hline \multirow[b]{3}{*}{ Surface } & \multirow[b]{3}{*}{ Adhesive } & \multirow[b]{3}{*}{$\begin{array}{l}\text { Days of } \\
\text { Exposure }\end{array}$} & \multicolumn{6}{|c|}{ Condition of Tamper Tape ${ }^{a}$} \\
\hline & & & \multicolumn{2}{|c|}{ Appearance } & \multicolumn{2}{|c|}{ Security Emblem } & \multicolumn{2}{|c|}{ Surface Adhesion } \\
\hline & & & $\begin{array}{c}\text { Window } \\
\text { Area }\end{array}$ & $\begin{array}{l}\text { Vinyl } \\
\text { Area }\end{array}$ & $\begin{array}{c}\text { Window } \\
\text { Area }\end{array}$ & $\begin{array}{l}\text { Vinyl } \\
\text { Area }\end{array}$ & $\begin{array}{c}\text { Window } \\
\text { Area }\end{array}$ & Overall \\
\hline \multirow[t]{2}{*}{$\begin{array}{l}\text { Polyester } \\
\text { Fiberglass } \\
\text { Board } \\
\text { (Cont.) }\end{array}$} & $\begin{array}{c}\text { epoxy } 1 \\
\text { epoxy } 2 \\
\text { polyurethane } \\
\text { acrylic }\end{array}$ & 35 & $\begin{array}{l}3 \\
4 \\
3 \\
2\end{array}$ & $\begin{array}{l}3 \\
4 \\
4 \\
4\end{array}$ & $\begin{array}{l}5 \\
5 \\
2 \\
2\end{array}$ & $\begin{array}{l}5 \\
5 \\
3 \\
3\end{array}$ & $\begin{array}{l}5 \\
5 \\
4 \\
3\end{array}$ & $\begin{array}{l}5 \\
5 \\
4 \\
3\end{array}$ \\
\hline & $\begin{array}{c}\text { epoxy } 1 \\
\text { epoxy } 2 \\
\text { polyurethane } \\
\text { acrylic }\end{array}$ & 42 & $\begin{array}{l}3 \\
4 \\
3 \\
2\end{array}$ & $\begin{array}{l}3 \\
4 \\
4 \\
3\end{array}$ & $\begin{array}{l}4 \\
5 \\
2 \\
2\end{array}$ & $\begin{array}{l}4 \\
5 \\
3 \\
3\end{array}$ & $\begin{array}{l}5 \\
5 \\
4 \\
3\end{array}$ & $\begin{array}{l}5 \\
5 \\
4 \\
3\end{array}$ \\
\hline \multirow[t]{7}{*}{$\begin{array}{l}\text { Mil. Spec. } \\
\text { PUb } \\
\text { Painted } \\
\text { Steel }\end{array}$} & $\begin{array}{c}\text { epoxy } 1 \\
\text { epoxy } 2 \\
\text { polyurethane } \\
\text { acrylic }\end{array}$ & 0 & $\begin{array}{l}5 \\
5 \\
5 \\
3\end{array}$ & $\begin{array}{c}5 \\
5 \\
5 \\
4-5\end{array}$ & $\begin{array}{l}5 \\
5 \\
5 \\
5\end{array}$ & $\begin{array}{l}5 \\
5 \\
5 \\
5\end{array}$ & $\begin{array}{l}5 \\
5 \\
5 \\
5\end{array}$ & $\begin{array}{l}5 \\
5 \\
5 \\
5\end{array}$ \\
\hline & $\begin{array}{c}\text { epoxy } 1 \\
\text { epoxy } 2 \\
\text { polyurethane } \\
\text { acrylic }\end{array}$ & 7 & $\begin{array}{c}4 \\
4-5 \\
5 \\
2\end{array}$ & $\begin{array}{c}4 \\
4-5 \\
5 \\
4\end{array}$ & $\begin{array}{l}5 \\
5 \\
5 \\
2 .\end{array}$ & $\begin{array}{l}5 \\
5 \\
5 \\
3\end{array}$ & $\begin{array}{l}5 \\
5 \\
5 \\
5\end{array}$ & $\begin{array}{l}5 \\
5 \\
5 \\
5\end{array}$ \\
\hline & $\begin{array}{c}\text { epoxy } 1 \\
\text { epoxy } 2 \\
\text { polyurethane } \\
\text { acrylic }\end{array}$ & 14 & $\begin{array}{c}4 \\
4 \\
4-5 \\
2\end{array}$ & $\begin{array}{c}4 \\
4 \\
4-5 \\
4\end{array}$ & $\begin{array}{l}5 \\
5 \\
5 \\
2\end{array}$ & $\begin{array}{l}5 \\
5 \\
5 \\
3\end{array}$ & $\begin{array}{l}5 \\
5 \\
5 \\
5\end{array}$ & $\begin{array}{l}5 \\
5 \\
5 \\
5\end{array}$ \\
\hline & $\begin{array}{c}\text { epoxy } 1 \\
\text { epoxy } 2 \\
\text { polyurethane } \\
\text { acrylic }\end{array}$ & 21 & $\begin{array}{c}4 \\
4 \\
4-5 \\
2\end{array}$ & $\begin{array}{c}4 \\
4 \\
4-5 \\
4\end{array}$ & $\begin{array}{l}5 \\
5 \\
5 \\
2\end{array}$ & $\begin{array}{l}5 \\
5 \\
5 \\
3\end{array}$ & $\begin{array}{l}5 \\
5 \\
5 \\
5\end{array}$ & $\begin{array}{l}5 \\
5 \\
5 \\
5\end{array}$ \\
\hline & $\begin{array}{c}\text { epoxy } 1 \\
\text { epoxy } 2 \\
\text { polyurethane } \\
\text { acrylic }\end{array}$ & 28 & $\begin{array}{l}4 \\
4 \\
3 \\
2\end{array}$ & $\begin{array}{c}4 \\
4 \\
4-5 \\
4\end{array}$ & $\begin{array}{l}5 \\
5 \\
4 \\
2\end{array}$ & $\begin{array}{l}5 \\
5 \\
4 \\
3\end{array}$ & $\begin{array}{l}5 \\
5 \\
5 \\
4\end{array}$ & $\begin{array}{c}5 \\
5 \\
5 \\
4-5\end{array}$ \\
\hline & $\begin{array}{c}\text { epoxy } 1 \\
\text { epoxy } 2 \\
\text { polyurethane } \\
\text { acrylic }\end{array}$ & 35 & $\begin{array}{l}4 \\
4 \\
3 \\
2\end{array}$ & $\begin{array}{c}3 \\
4 \\
4-5 \\
4\end{array}$ & $\begin{array}{l}4 \\
5 \\
4 \\
2\end{array}$ & $\begin{array}{l}4 \\
5 \\
4 \\
3\end{array}$ & $\begin{array}{l}5 \\
5 \\
4 \\
4\end{array}$ & $\begin{array}{l}5 \\
5 \\
4-5 \\
4-5\end{array}$ \\
\hline & $\begin{array}{c}\text { epoxy } 1 \\
\text { epoxy } 2 \\
\text { polyurethane } \\
\text { acrylic }\end{array}$ & 42 & $\begin{array}{l}3 \\
4 \\
3 \\
2\end{array}$ & $\begin{array}{c}3 \\
4 \\
4-5 \\
3\end{array}$ & $\begin{array}{l}4 \\
5 \\
3 \\
2\end{array}$ & $\begin{array}{l}4 \\
4 \\
3 \\
3\end{array}$ & $\begin{array}{l}5 \\
5 \\
4 \\
4\end{array}$ & $\begin{array}{c}5 \\
5 \\
4-5 \\
4\end{array}$ \\
\hline
\end{tabular}

a See Table 3.8 for a description of the numerical ranking code for assessing tamper tapes.

b Polyurethane 
Table 4.7. Evaluation of Confirm ${ }^{\circledR} 1500$ With Primer Tamper Tapes Applied with Candidate Rapid-Set Adhesives and Aged in QUV Cabinet (UV, $60^{\circ} \mathrm{C}$; Condensing Humidity, $\left.40^{\circ} \mathrm{C}\right)$

\begin{tabular}{|c|c|c|c|c|c|c|c|c|}
\hline \multirow[b]{3}{*}{ Surface } & \multirow[b]{3}{*}{ Adhesive } & \multirow[b]{3}{*}{$\begin{array}{c}\text { Days of } \\
\text { Exposure }\end{array}$} & \multicolumn{6}{|c|}{ Condition of Tamper Tape ${ }^{a}$} \\
\hline & & & \multicolumn{2}{|c|}{ Appearance } & \multirow{2}{*}{$\begin{array}{c}\text { Security } \\
\text { Window } \\
\text { Area } \\
\end{array}$} & \multirow{2}{*}{$\begin{array}{c}\text { Emblem } \\
\text { Vinyl } \\
\text { Area } \\
\end{array}$} & \multicolumn{2}{|c|}{ Surface Adhesion } \\
\hline & & & $\begin{array}{c}\text { Window } \\
\text { Area }\end{array}$ & $\begin{array}{l}\text { Vinyl } \\
\text { Area } \\
\end{array}$ & & & $\begin{array}{c}\text { Window } \\
\text {.Area } \\
\end{array}$ & Overall \\
\hline Aluminum & $\begin{array}{c}\text { epoxy } 1 \\
\text { epoxy } 2 \\
\text { polyurethane } \\
\text { acrylic }\end{array}$ & 0 & $\begin{array}{l}4-5 \\
5 \\
4-5 \\
3-4\end{array}$ & $\begin{array}{c}4-5 \\
5 \\
4-5 \\
5\end{array}$ & $\begin{array}{l}5 \\
5 \\
5 \\
5\end{array}$ & $\begin{array}{l}5 \\
5 \\
5 \\
5\end{array}$ & $\begin{array}{l}5 \\
5 \\
5 \\
5\end{array}$ & $\begin{array}{l}5 \\
5 \\
5 \\
5\end{array}$ \\
\hline & $\begin{array}{c}\text { epoxy } 1 \\
\text { epoxy } 2 \\
\text { polyurethane } \\
\text { acrylic }\end{array}$ & 7 & $\begin{array}{c}3-4 \\
5 \\
4 \\
3\end{array}$ & $\begin{array}{c}3-4 \\
5 \\
4 \\
5\end{array}$ & $\begin{array}{l}5 \\
5 \\
5 \\
3\end{array}$ & $\begin{array}{l}5 \\
5 \\
5 \\
4\end{array}$ & $\begin{array}{l}5 \\
5 \\
5 \\
5\end{array}$ & $\begin{array}{l}5 \\
5 \\
5 \\
5\end{array}$ \\
\hline & $\begin{array}{c}\text { epoxy } 1 \\
\text { epoxy } 2 \\
\text { polyurethane } \\
\text { acrylic }\end{array}$ & 14 & $\begin{array}{c}3-4 \\
4-5 \\
4 \\
3\end{array}$ & $\begin{array}{c}3-4 \\
4-5 \\
4 \\
5\end{array}$ & $\begin{array}{l}5 \\
5 \\
5 \\
3\end{array}$ & $\begin{array}{l}5 \\
5 \\
5 \\
4\end{array}$ & $\begin{array}{l}5 \\
5 \\
5 \\
5\end{array}$ & $\begin{array}{l}5 \\
5 \\
5 \\
5\end{array}$ \\
\hline & $\begin{array}{c}\text { epoxy } 1 \\
\text { epoxy } 2 \\
\text { polyurethane } \\
\text { acrylic }\end{array}$ & 21 & $\begin{array}{c}3-4 \\
4-5 \\
4 \\
3\end{array}$ & $\begin{array}{c}3-4 \\
4-5 \\
4 \\
5\end{array}$ & $\begin{array}{l}5 \\
5 \\
5 \\
3\end{array}$ & $\begin{array}{l}5 \\
5 \\
5 \\
4\end{array}$ & $\begin{array}{l}5 \\
5 \\
5 \\
5\end{array}$ & $\begin{array}{l}5 \\
5 \\
5 \\
5\end{array}$ \\
\hline & $\begin{array}{c}\text { epoxy } 1 \\
\text { epoxy } 2 \\
\text { polyurethanc } \\
\text { acrylic }\end{array}$ & 28 & $\begin{array}{c}3-4 \\
4-5 \\
4 \\
3\end{array}$ & $\begin{array}{c}3-4 \\
4-5 \\
4 \\
5\end{array}$ & $\begin{array}{l}4 \\
5 \\
4 \\
3\end{array}$ & $\begin{array}{l}5 \\
5 \\
4 \\
4\end{array}$ & $\begin{array}{l}5 \\
5 \\
5 \\
5\end{array}$ & $\begin{array}{l}5 \\
5 \\
5 \\
5\end{array}$ \\
\hline & $\begin{array}{l}\text { epoxy } 1 \\
\text { epoxy } 2 \\
\text { polyurethane } \\
\text { acrylic }\end{array}$ & 35 & $\begin{array}{c}3-4 \\
4-5 \\
4 \\
3\end{array}$ & $\begin{array}{c}3 \\
4-5 \\
4 \\
5\end{array}$ & $\begin{array}{l}4 \\
5 \\
4 \\
3\end{array}$ & $\begin{array}{l}4 \\
5 \\
4 \\
4\end{array}$ & $\begin{array}{l}5 \\
5 \\
5 \\
4\end{array}$ & $\begin{array}{l}5 \\
5 \\
5 \\
4\end{array}$ \\
\hline . & $\begin{array}{l}\text { epoxy } 1 \\
\text { epoxy } 2 \\
\text { polyurethane } \\
\text { acrylic }\end{array}$ & 42 & $\begin{array}{c}3 \\
4-5 \\
3 \\
2\end{array}$ & $\begin{array}{c}2 \\
4-5 \\
3 \\
3\end{array}$ & $\begin{array}{l}4 \\
5 \\
4 \\
1\end{array}$ & $\begin{array}{l}4 \\
5 \\
4 \\
3\end{array}$ & $\begin{array}{l}5 \\
5 \\
5 \\
4\end{array}$ & $\begin{array}{l}5 \\
5 \\
5 \\
4\end{array}$ \\
\hline Steel & $\begin{array}{c}\text { epoxy } 1 \\
\text { epoxy } 2 \\
\text { polyurethane } \\
\text { acrylic }^{b}\end{array}$ & 0 & $\begin{array}{l}4-5 \\
5 \\
5 \\
5\end{array}$ & $\begin{array}{l}4-5 \\
5 \\
5 \\
5\end{array}$ & $\begin{array}{l}5 \\
5 \\
5 \\
5\end{array}$ & $\begin{array}{l}5 \\
5 \\
5 \\
5\end{array}$ & $\begin{array}{l}5 \\
5 \\
5 \\
5\end{array}$ & $\begin{array}{l}5 \\
5 \\
5 \\
5\end{array}$ \\
\hline & $\begin{array}{c}\text { epoxy } 1 \\
\text { epoxy } 2 \\
\text { polyurethanc } \\
\text { acrylic }\end{array}$ & 7 & $\begin{array}{c}3-4 \\
5 \\
4-5 \\
4\end{array}$ & $\begin{array}{c}3-4 \\
5 \\
4-5 \\
5\end{array}$ & $\begin{array}{l}5 \\
5 \\
5 \\
3\end{array}$ & $\begin{array}{l}5 \\
5 \\
5 \\
5\end{array}$ & $\begin{array}{l}5 \\
5 \\
5 \\
5\end{array}$ & $\begin{array}{l}5 \\
5 \\
5 \\
5\end{array}$ \\
\hline
\end{tabular}

a See Table 3.8 for a description of the numerical ranking code for assessing tamper tapes.

$\mathrm{b}$ A different rapid-set acrylic adhesive was used on this surface compared to the others. 
Table 4.7. (Continued)

\begin{tabular}{|c|c|c|c|c|c|c|c|c|}
\hline \multirow[b]{3}{*}{ Surface } & \multirow[b]{3}{*}{ Adhesive } & \multirow[b]{3}{*}{$\begin{array}{c}\text { Days of } \\
\text { Exposure }\end{array}$} & \multicolumn{6}{|c|}{ Condition of Tamper Tape ${ }^{a}$} \\
\hline & & & \multicolumn{2}{|c|}{ Appearance } & \multicolumn{2}{|c|}{ Security Emblem } & \multirow{2}{*}{$\begin{array}{c}\text { Surface } \\
\text { Window } \\
\text { Area }\end{array}$} & \multirow{2}{*}{$\begin{array}{r}\text { Adhesion } \\
\text { Overall } \\
\end{array}$} \\
\hline & & & $\begin{array}{c}\text { Window } \\
\text { Area }\end{array}$ & $\begin{array}{l}\text { Vinyl } \\
\text { Area }\end{array}$ & $\begin{array}{c}\text { Window } \\
\text { Area }\end{array}$ & $\begin{array}{l}\text { Vinyl } \\
\text { Area }\end{array}$ & & \\
\hline \multirow[t]{5}{*}{$\begin{array}{l}\text { Steel } \\
\text { (Cont.) }\end{array}$} & $\begin{array}{c}\text { epoxy } 1 \\
\text { epoxy } 2 \\
\text { polyurethane } \\
\text { acrylic } b\end{array}$ & 14 & $\begin{array}{c}3-4 \\
4-5 \\
4 \\
4\end{array}$ & $\begin{array}{c}3-4 \\
4-5 \\
4 \\
5\end{array}$ & $\begin{array}{l}5 \\
5 \\
5 \\
4\end{array}$ & $\begin{array}{l}5 \\
5 \\
5 \\
5\end{array}$ & $\begin{array}{l}5 \\
5 \\
5 \\
5\end{array}$ & $\begin{array}{l}5 \\
5 \\
5 \\
5\end{array}$ \\
\hline & $\begin{array}{c}\text { epoxy } 1 \\
\text { epoxy } 2 \\
\text { polyurethane } \\
\text { acrylic }\end{array}$ & 21 & $\begin{array}{c}3-4 \\
4-5 \\
4 \\
4\end{array}$ & $\begin{array}{c}3-4 \\
4-5 \\
4 \\
5\end{array}$ & $\begin{array}{l}5 \\
5 \\
5 \\
4\end{array}$ & $\begin{array}{l}5 \\
5 \\
5 \\
5\end{array}$ & $\begin{array}{l}5 \\
5 \\
5 \\
5\end{array}$ & $\begin{array}{l}5 \\
5 \\
5 \\
5\end{array}$ \\
\hline & $\begin{array}{c}\text { epoxy } 1 \\
\text { epoxy } 2 \\
\text { polyurethane } \\
\text { acrylic } b\end{array}$ & 28 & $\begin{array}{c}3-4 \\
4-5 \\
4 \\
4\end{array}$ & $\begin{array}{c}3 \\
4-5 \\
4 \\
5\end{array}$ & $\begin{array}{l}4 \\
5 \\
5 \\
4\end{array}$ & $\begin{array}{l}5 \\
5 \\
5 \\
5\end{array}$ & $\begin{array}{l}5 \\
5 \\
5 \\
5\end{array}$ & $\begin{array}{l}5 \\
5 \\
5 \\
5\end{array}$ \\
\hline & $\begin{array}{c}\text { epoxy } 1 \\
\text { epoxy } 2 \\
\text { polyurethane } \\
\text { acrylic }^{b}\end{array}$ & 35 & $\begin{array}{c}3-4 \\
4-5 \\
4 \\
4\end{array}$ & $\begin{array}{c}3 \\
4-5 \\
4 \\
5\end{array}$ & $\begin{array}{l}4 \\
5 \\
5 \\
4\end{array}$ & $\begin{array}{l}3 \\
5 \\
5 \\
5\end{array}$ & $\begin{array}{l}5 \\
5 \\
5 \\
5\end{array}$ & $\begin{array}{l}5 \\
5 \\
5 \\
5\end{array}$ \\
\hline & $\begin{array}{c}\text { epoxy } 1 \\
\text { epoxy } 2 \\
\text { polyurethane } \\
\text { acrylic }^{b}\end{array}$ & 42 & $\begin{array}{c}3 \\
4-5 \\
3 \\
4\end{array}$ & $\begin{array}{c}2 \\
4-5 \\
3 \\
3\end{array}$ & $\begin{array}{l}4 \\
5 \\
3 \\
4\end{array}$ & $\begin{array}{l}3 \\
5 \\
4 \\
5\end{array}$ & $\begin{array}{l}5 \\
5 \\
5 \\
5\end{array}$ & $\begin{array}{l}5 \\
5 \\
5 \\
5\end{array}$ \\
\hline \multirow[t]{5}{*}{$\begin{array}{l}\text { Polyester } \\
\text { Fiberglass } \\
\text { Board }\end{array}$} & $\begin{array}{c}\text { epoxy } 1 \\
\text { epoxy } 2 \\
\text { polyurethane } \\
\text { acrylic }\end{array}$ & 0 & $\begin{array}{l}5 \\
5 \\
5 \\
2\end{array}$ & $\begin{array}{l}5 \\
5 \\
5 \\
2\end{array}$ & $\begin{array}{l}5 \\
5 \\
5 \\
3\end{array}$ & $\begin{array}{l}5 \\
5 \\
5 \\
3\end{array}$ & $\begin{array}{l}5 \\
5 \\
5 \\
5\end{array}$ & $\begin{array}{l}5 \\
5 \\
5 \\
5\end{array}$ \\
\hline & $\begin{array}{c}\text { epoxy } 1 \\
\text { epoxy } 2 \\
\text { polyurethane } \\
\text { acrylic }\end{array}$ & 7 & $\begin{array}{c}3-4 \\
5 \\
4-5 \\
2\end{array}$ & $\begin{array}{c}3-4 \\
5 \\
4-5 \\
2\end{array}$ & $\begin{array}{l}5 \\
5 \\
5 \\
2\end{array}$ & $\begin{array}{l}5 \\
5 \\
5 \\
3\end{array}$ & $\begin{array}{l}5 \\
5 \\
5 \\
5\end{array}$ & $\begin{array}{l}5 \\
5 \\
5 \\
5\end{array}$ \\
\hline & $\begin{array}{c}\text { epoxy } 1 \\
\text { epoxy } 2 \\
\text { polyurethane } \\
\text { acrylic }\end{array}$ & 14 & $\begin{array}{c}3-4 \\
4-5 \\
4 \\
2\end{array}$ & $\begin{array}{c}3-4 \\
4-5 \\
4 \\
2\end{array}$ & $\begin{array}{l}5 \\
5 \\
5 \\
2\end{array}$ & $\begin{array}{l}5 \\
5 \\
5 \\
3\end{array}$ & $\begin{array}{l}5 \\
5 \\
5 \\
5\end{array}$ & $\begin{array}{l}5 \\
5 \\
5 \\
5\end{array}$ \\
\hline & $\begin{array}{c}\text { epoxy } 1 \\
\text { epoxy } 2 \\
\text { polyurethane } \\
\text { acrylic }\end{array}$ & 21 & $\begin{array}{c}3-4 \\
4-5 \\
4 \\
2\end{array}$ & $\begin{array}{c}3-4 \\
4-5 \\
4 \\
2\end{array}$ & $\begin{array}{l}5 \\
5 \\
5 \\
2\end{array}$ & $\begin{array}{l}5 \\
5 \\
5 \\
3\end{array}$ & $\begin{array}{l}5 \\
5 \\
5 \\
5\end{array}$ & $\begin{array}{l}5 \\
5 \\
5 \\
5\end{array}$ \\
\hline & $\begin{array}{l}\text { epoxy } 1 \\
\text { epoxy } 2 \\
\text { polyurethane } \\
\text { acrylic }\end{array}$ & .28 & $\begin{array}{c}3-4 \\
4-5 \\
3 \\
1\end{array}$ & $\begin{array}{c}3-4 \\
4-5 \\
4 \\
2\end{array}$ & $\begin{array}{l}4 \\
5 \\
5 \\
2\end{array}$ & $\begin{array}{l}5 \\
5 \\
5 \\
3\end{array}$ & $\begin{array}{l}5 \\
5 \\
5 \\
4\end{array}$ & $\begin{array}{l}5 \\
5 \\
5 \\
4\end{array}$ \\
\hline
\end{tabular}

a See Table 3.8 for a description of the numerical ranking code for assessing tamper tapes.

b A different rapid-set acrylic adhesive was used on this surface compared to the others. 
Table 4.7. (Continued)

\begin{tabular}{|c|c|c|c|c|c|c|c|c|}
\hline \multirow[b]{3}{*}{ Surface } & \multirow[b]{3}{*}{ Adhesive } & \multirow[b]{3}{*}{$\begin{array}{c}\text { Days of } \\
\text { Exposure }\end{array}$} & \multicolumn{6}{|c|}{ Condition of Tamper Tape ${ }^{a}$} \\
\hline & & & \multicolumn{2}{|c|}{ Appearance } & \multicolumn{2}{|c|}{ Security Emblem } & \multicolumn{2}{|c|}{ Surface Adhesion } \\
\hline & & & $\begin{array}{c}\text { Window } \\
\text { Area }\end{array}$ & $\begin{array}{l}\text { Vinyl } \\
\text { Area } \\
\end{array}$ & $\begin{array}{c}\text { Window } \\
\text { Area } \\
\end{array}$ & $\begin{array}{l}\text { Vinyl } \\
\text { Area }\end{array}$ & $\begin{array}{c}\text { Window } \\
\text { Area } \\
\end{array}$ & Overall \\
\hline \multirow[t]{2}{*}{$\begin{array}{l}\text { Polyester } \\
\text { Fiberglass } \\
\text { Board } \\
\text { (Cont.) }\end{array}$} & $\begin{array}{c}\text { epoxy } 1 \\
\text { epoxy } 2 \\
\text { polyurethane } \\
\text { acrylic }\end{array}$ & 35 & $\begin{array}{c}3-4 \\
4-5 \\
4 \\
1\end{array}$ & $\begin{array}{c}3 \\
4-5 \\
4 \\
2\end{array}$ & $\begin{array}{l}3 \\
5 \\
5 \\
2\end{array}$ & $\begin{array}{l}4 \\
5 \\
5 \\
3\end{array}$ & $\begin{array}{l}5 \\
5 \\
5 \\
4\end{array}$ & $\begin{array}{l}5 \\
5 \\
5 \\
4\end{array}$ \\
\hline & $\begin{array}{c}\text { epoxy. } 1 \\
\text { epoxy } 2 \\
\text { polyurethane } \\
\text { acrylic }\end{array}$ & 42 & $\begin{array}{c}3 \\
4-5 \\
3 \\
1\end{array}$ & $\begin{array}{c}2 \\
4-5 \\
3 \\
2\end{array}$ & $\begin{array}{l}3 \\
4 \\
4 \\
1\end{array}$ & $\begin{array}{l}4 \\
5 \\
3 \\
2\end{array}$ & $\begin{array}{l}5 \\
5 \\
5 \\
3\end{array}$ & $\begin{array}{l}5 \\
5 \\
5 \\
3\end{array}$ \\
\hline \multirow[t]{7}{*}{$\begin{array}{l}\text { Mil. Spec. } \\
\text { PUc } \\
\text { Painted } \\
\text { Steel }\end{array}$} & $\begin{array}{c}\text { epoxy } 1 \\
\text { epoxy } 2 \\
\text { polyurethanc } \\
\text { acrylic }\end{array}$ & 0 & $\begin{array}{c}4-5 \\
5 \\
5 \\
2\end{array}$ & $\begin{array}{c}4-5 \\
5 \\
5 \\
2\end{array}$ & $\begin{array}{l}5 \\
5 \\
5 \\
1\end{array}$ & $\begin{array}{l}5 \\
5 \\
5 \\
3\end{array}$ & $\begin{array}{l}5 \\
5 \\
5 \\
5\end{array}$ & $\begin{array}{l}5 \\
5 \\
5 \\
5\end{array}$ \\
\hline & $\begin{array}{c}\text { epoxy } 1 \\
\text { epoxy } 2 \\
\text { polyurethane } \\
\text { acrylic }\end{array}$ & 7 & $\begin{array}{c}3-4 \\
5 \\
4-5 \\
2\end{array}$ & $\begin{array}{c}3-4 \\
5 \\
4-5 \\
2\end{array}$ & $\begin{array}{l}5 \\
5 \\
5 \\
1\end{array}$ & $\begin{array}{l}5 \\
5 \\
5 \\
3\end{array}$ & $\begin{array}{l}5 \\
5 \\
5 \\
5\end{array}$ & $\begin{array}{r}5 \\
5 \\
5 \\
5\end{array}$ \\
\hline & $\begin{array}{l}\text { epoxy } 1 \\
\text { epoxy } 2 \\
\text { polyurethane } \\
\text { acrylic }\end{array}$ & 14 & $\begin{array}{c}3-4 \\
4-5 \\
4 \\
2\end{array}$ & $\begin{array}{c}3-4 \\
4-5 \\
4-5 \\
2\end{array}$ & $\begin{array}{l}5 \\
5 \\
5 \\
1\end{array}$ & $\begin{array}{l}5 \\
5 \\
5 \\
3\end{array}$ & $\begin{array}{l}5 \\
5 \\
5 \\
5\end{array}$ & $\begin{array}{l}5 \\
5 \\
5 \\
5\end{array}$ \\
\hline & $\begin{array}{c}\text { epoxy } 1 \\
\text { epoxy } 2 \\
\text { polyurethane } \\
\text { acrylic }\end{array}$ & 21 & $\begin{array}{c}3-4 \\
4-5 \\
4 \\
2\end{array}$ & $\begin{array}{c}3-4 \\
4-5 \\
4-5 \\
2\end{array}$ & $\begin{array}{l}5 \\
5 \\
5 \\
1\end{array}$ & $\begin{array}{l}5 \\
5 \\
5 \\
3\end{array}$ & $\begin{array}{l}5 \\
5 \\
5 \\
5\end{array}$ & $\begin{array}{l}5 \\
5 \\
5 \\
5\end{array}$ \\
\hline & $\begin{array}{c}\text { cpoxy } 1 \\
\text { epoxy } 2 \\
\text { polyurethane } \\
\text { acrylic }\end{array}$ & 28 & $\begin{array}{c}3-4 \\
4-5 \\
4 \\
2\end{array}$ & $\begin{array}{c}3-4 \\
4-5 \\
4-5 \\
2\end{array}$ & $\begin{array}{l}3 \\
5 \\
3 \\
1\end{array}$ & $\begin{array}{l}4 \\
5 \\
3 \\
2\end{array}$ & $\begin{array}{l}5 \\
5 \\
5 \\
4\end{array}$ & $\begin{array}{l}5 \\
5 \\
5 \\
4\end{array}$ \\
\hline & $\begin{array}{c}\text { epoxy } 1 \\
\text { epoxy } 2 \\
\text { polyurethane } \\
\text { acrylic }\end{array}$ & 35 & $\begin{array}{c}3-4 \\
4-5 \\
4 \\
2\end{array}$ & $\begin{array}{c}3 \\
4-5 \\
4-5 \\
2\end{array}$ & $\begin{array}{l}3 \\
5 \\
3 \\
1\end{array}$ & $\begin{array}{l}4 \\
5 \\
3 \\
2\end{array}$ & $\begin{array}{l}5 \\
5 \\
5 \\
4\end{array}$ & $\begin{array}{l}5 \\
5 \\
5 \\
4\end{array}$ \\
\hline & $\begin{array}{c}\text { epoxy } 1 \\
\text { epoxy } 2 \\
\text { polyurethane } \\
\text { acrylic }\end{array}$ & 42 & $\begin{array}{c}3 \\
4-5 \\
3 \\
1\end{array}$ & $\begin{array}{c}2 \\
4-5 \\
4 \\
2\end{array}$ & $\begin{array}{l}3 \\
5 \\
3 \\
1\end{array}$ & $\begin{array}{l}3 \\
5 \\
3 \\
2\end{array}$ & $\begin{array}{l}5 \\
5 \\
5 \\
3\end{array}$ & $\begin{array}{l}5 \\
5 \\
5 \\
3\end{array}$ \\
\hline
\end{tabular}

a See Table 3.8 for a description of the numerical ranking code for assessing tamper tapes.

c Polyurethane 
Table 4.8. Evaluation of Confirm ${ }^{\circledR} 1300$ Tamper Tapes Applied with Candidate Rapid-Set Adhesives and Aged in QUV Cabinet (UV, $60^{\circ} \mathrm{C}$; Condensing Humidity, $40^{\circ} \mathrm{C}$ )

\begin{tabular}{|c|c|c|c|c|c|c|c|c|}
\hline \multirow[b]{3}{*}{ Surface } & \multirow[b]{3}{*}{ Adhesive } & \multirow[b]{3}{*}{$\begin{array}{c}\text { Days of } \\
\text { Exposure }\end{array}$} & \multicolumn{6}{|c|}{ Condition of Tamper Tape ${ }^{a}$} \\
\hline & & & \multicolumn{2}{|c|}{ Appearance } & \multicolumn{2}{|c|}{ Security Emblem } & \multicolumn{2}{|c|}{ Surface Adhesion } \\
\hline & & & $\begin{array}{c}\text { Window } \\
\text { Area }\end{array}$ & $\begin{array}{l}\text { Vinyl } \\
\text { Area } \\
\end{array}$ & $\begin{array}{c}\text { Window } \\
\text { Area }\end{array}$ & $\begin{array}{l}\text { Vinyl } \\
\text { Area } \\
\end{array}$ & $\begin{array}{c}\text { Window } \\
\text { Area }\end{array}$ & Overall \\
\hline \multirow[t]{7}{*}{ Aluminum } & $\begin{array}{c}\text { epoxy } 1 \\
\text { epoxy } 2 \\
\text { polyurethane } \\
\text { acrylic }\end{array}$ & 0 & $\begin{array}{l}5 \\
5 \\
5 \\
5\end{array}$ & $\begin{array}{l}5 \\
5 \\
5 \\
5\end{array}$ & $\begin{array}{l}5 \\
5 \\
5 \\
5\end{array}$ & $\begin{array}{l}5 \\
5 \\
5 \\
5\end{array}$ & $\begin{array}{l}5 \\
5 \\
5 \\
5\end{array}$ & $\begin{array}{l}5 \\
5 \\
5 \\
5\end{array}$ \\
\hline & $\begin{array}{c}\text { epoxy } 1 \\
\text { epoxy } 2 \\
\text { polyurethane } \\
\text { acrylic }\end{array}$ & 7 & $\begin{array}{l}4-5 \\
5 \\
5 \\
5\end{array}$ & $\begin{array}{l}4-5 \\
5 \\
5 \\
5\end{array}$ & $\begin{array}{l}5 \\
5 \\
5 \\
5\end{array}$ & $\begin{array}{l}5 \\
5 \\
5 \\
5\end{array}$ & $\begin{array}{l}5 \\
5 \\
5 \\
5\end{array}$ & $\begin{array}{l}5 \\
5 \\
5 \\
5\end{array}$ \\
\hline & $\begin{array}{c}\text { epoxy } 1 \\
\text { epoxy } 2 \\
\text { polyurethane } \\
\text { acrylic }\end{array}$ & 14 & $\begin{array}{l}4-5 \\
4-5 \\
4-5 \\
4-5\end{array}$ & $\begin{array}{l}4-5 \\
4-5 \\
4-5 \\
4-5\end{array}$ & $\begin{array}{l}5 \\
5 \\
5 \\
5\end{array}$ & $\begin{array}{l}5 \\
5 \\
5 \\
5\end{array}$ & $\begin{array}{l}5 \\
5 \\
5 \\
5\end{array}$ & $\begin{array}{l}5 \\
5 \\
5 \\
5\end{array}$ \\
\hline & $\begin{array}{c}\text { epoxy } 1 \\
\text { epoxy } 2 \\
\text { polyurethane } \\
\text { acrylic }\end{array}$ & 21 & $\begin{array}{l}4-5 \\
4-5 \\
4-5 \\
4-5\end{array}$ & $\begin{array}{l}4-5 \\
4-5 \\
4-5 \\
4-5\end{array}$ & $\begin{array}{l}5 \\
5 \\
5 \\
5\end{array}$ & $\begin{array}{l}5 \\
5 \\
5 \\
5\end{array}$ & $\begin{array}{l}5 \\
5 \\
5 \\
5\end{array}$ & $\begin{array}{l}5 \\
5 \\
5 \\
5\end{array}$ \\
\hline & $\begin{array}{c}\text { epoxy } 1 \\
\text { epoxy } 2 \\
\text { polyurethane } \\
\text { acrylic }\end{array}$ & 28 & $\begin{array}{c}4 \\
4-5 \\
4 \\
4-5\end{array}$ & $\begin{array}{l}4 \\
4-5 \\
4-5 \\
4-5\end{array}$ & $\begin{array}{l}5 \\
5 \\
5 \\
5\end{array}$ & $\begin{array}{l}5 \\
5 \\
5 \\
5\end{array}$ & $\begin{array}{l}5 \\
5 \\
5 \\
5\end{array}$ & $\begin{array}{l}5 \\
5 \\
5 \\
5\end{array}$ \\
\hline & $\begin{array}{c}\text { epoxy } 1 \\
\text { epoxy } 2 \\
\text { polyurethane } \\
\text { acrylic }\end{array}$ & 35 & $\begin{array}{c}4 \\
4-5 \\
4 \\
4-5\end{array}$ & $\begin{array}{l}3 \\
4-5 \\
4-5 \\
4-5\end{array}$ & $\begin{array}{l}5 \\
5 \\
5 \\
4\end{array}$ & $\begin{array}{l}5 \\
5 \\
5 \\
5\end{array}$ & $\begin{array}{l}5 \\
5 \\
4 \\
5\end{array}$ & $\begin{array}{l}5 \\
5 \\
4 \\
5\end{array}$ \\
\hline & $\begin{array}{c}\text { epoxy } 1 \\
\text { epoxy } 2 \\
\text { polyurethane } \\
\text { acrylic }\end{array}$ & 42 & $\begin{array}{c}3 \\
4-5 \\
3 \\
4-5\end{array}$ & $\begin{array}{l}3 \\
4-5 \\
4-5 \\
4-5\end{array}$ & $\begin{array}{l}4 \\
5 \\
5 \\
4\end{array}$ & $\begin{array}{l}4 \\
5 \\
5 \\
4\end{array}$ & $\begin{array}{l}5 \\
5 \\
5 \\
5\end{array}$ & $\begin{array}{l}5 \\
5 \\
5 \\
5\end{array}$ \\
\hline \multirow[t]{2}{*}{ Steel } & $\begin{array}{c}\text { epoxy } 1 \\
\text { epoxy } 2 \\
\text { polyurethane } \\
\text { acrylic }\end{array}$ & 0 & $\begin{array}{c}4-5 \\
5 \\
5 \\
5\end{array}$ & $\begin{array}{l}5 \\
5 \\
5 \\
5\end{array}$ & $\begin{array}{l}5 \\
5 \\
5 \\
5\end{array}$ & $\begin{array}{l}5 \\
5 \\
5 \\
5\end{array}$ & $\begin{array}{l}5 \\
5 \\
5 \\
5\end{array}$ & $\begin{array}{l}5 \\
5 \\
5 \\
5\end{array}$ \\
\hline & $\begin{array}{c}\text { epoxy } 1 \\
\text { epoxy } 2 \\
\text { polyurethane } \\
\text { acrylic }\end{array}$ & 7 & $\begin{array}{l}3-4 \\
5 \\
5 \\
5\end{array}$ & $\begin{array}{l}4 \\
5 \\
5 \\
5\end{array}$ & $\begin{array}{l}5 \\
5 \\
5 \\
5\end{array}$ & $\begin{array}{l}5 \\
5 \\
5 \\
5\end{array}$ & $\begin{array}{l}5 \\
5 \\
5 \\
5\end{array}$ & $\begin{array}{l}5 \\
5 \\
5 \\
5\end{array}$ \\
\hline
\end{tabular}

a See Table 3.8 for a description of the numerical ranking code for assessing tamper tapes. 
Table 4.8. (Continued)

\begin{tabular}{|c|c|c|c|c|c|c|c|c|}
\hline \multirow[b]{3}{*}{ Surface } & \multirow[b]{3}{*}{ Adhesive } & \multirow[b]{3}{*}{$\begin{array}{c}\text { Days of } \\
\text { Exposure }\end{array}$} & \multicolumn{6}{|c|}{ Condition of Tamper Tape ${ }^{a}$} \\
\hline & & & \multicolumn{2}{|c|}{ Appearance } & \multicolumn{2}{|c|}{ Security Emblem } & \multicolumn{2}{|c|}{ Surface Adhesion } \\
\hline & & & $\begin{array}{c}\text { Window } \\
\text { Area }\end{array}$ & $\begin{array}{l}\text { Vinyl } \\
\text { Area } \\
\end{array}$ & $\begin{array}{c}\text { Window } \\
\text { Area } \\
\end{array}$ & $\begin{array}{l}\text { Vinyl } \\
\text { Area }\end{array}$ & $\begin{array}{c}\text { Window } \\
\text { Area }\end{array}$ & Overall \\
\hline \multirow[t]{5}{*}{$\begin{array}{l}\text { Steel } \\
\text { (Cont.) }\end{array}$} & $\begin{array}{c}\text { epoxy } 1 \\
\text { epoxy } 2 \\
\text { polyurethane } \\
\text { acrylic }\end{array}$ & 14 & $\begin{array}{l}3-4 \\
4-5 \\
4-5 \\
4-5\end{array}$ & $\begin{array}{l}4 \\
4-5 \\
4-5 \\
4-5\end{array}$ & $\begin{array}{l}5 \\
5 \\
5 \\
5\end{array}$ & $\begin{array}{l}5 \\
5 \\
5 \\
5\end{array}$ & $\begin{array}{l}5 \\
5 \\
5 \\
5\end{array}$ & $\begin{array}{l}5 \\
5 \\
5 \\
5\end{array}$ \\
\hline & $\begin{array}{c}\text { epoxy } 1 \\
\text { epoxy } 2 \\
\text { polyurethane } \\
\text { acrylic }\end{array}$ & 21 & $\begin{array}{l}3-4 \\
4-5 \\
4-5 \\
4-5\end{array}$ & $\begin{array}{l}4 \\
4-5 \\
4-5 \\
4-5\end{array}$ & $\begin{array}{l}5 \\
5 \\
5 \\
5\end{array}$ & $\begin{array}{l}5 \\
5 \\
5 \\
5\end{array}$ & $\begin{array}{l}5 \\
5 \\
5 \\
5\end{array}$ & $\begin{array}{l}5 \\
5 \\
5 \\
5\end{array}$ \\
\hline & $\begin{array}{c}\text { epoxy } 1 \\
\text { epoxy } 2 \\
\text { polyurethane } \\
\text { acrylic }\end{array}$ & 28 & $\begin{array}{c}3-4 \\
4-5 \\
4 \\
4-5\end{array}$ & $\begin{array}{c}4 \\
4-5 \\
4-5 \\
4-5\end{array}$ & $\begin{array}{l}4 \\
5 \\
5 \\
5\end{array}$ & $\begin{array}{l}5 \\
5 \\
5 \\
5\end{array}$ & $\begin{array}{l}5 \\
5 \\
5 \\
5\end{array}$ & $\begin{array}{l}5 \\
5 \\
5 \\
5\end{array}$ \\
\hline & $\begin{array}{c}\text { epoxy } 1 \\
\text { epoxy } 2 \\
\text { polyurethane } \\
\text { acrylic }\end{array}$ & 35 & $\begin{array}{c}3-4 \\
4-5 \\
4 \\
4-5\end{array}$ & $\begin{array}{l}3 \\
4-5 \\
4-5 \\
4-5\end{array}$ & $\begin{array}{l}4 \\
5 \\
4 \\
5\end{array}$ & $\begin{array}{l}5 \\
5 \\
5 \\
5\end{array}$ & $\begin{array}{l}5 \\
5 \\
4 \\
5\end{array}$ & $\begin{array}{l}5 \\
5 \\
4 \\
5\end{array}$ \\
\hline & $\begin{array}{c}\text { epoxy } 1 \\
\text { epoxy } 2 \\
\text { polyurethane } \\
\text { acrylic }\end{array}$ & 42 & $\begin{array}{c}3 \\
4-5 \\
3 \\
4-5\end{array}$ & $\begin{array}{l}3 \\
4-5 \\
4-5 \\
4-5\end{array}$ & $\begin{array}{l}4 \\
5 \\
4 \\
3\end{array}$ & $\begin{array}{l}3 \\
5 \\
4 \\
4\end{array}$ & $\begin{array}{l}5 \\
5 \\
5 \\
5\end{array}$ & $\begin{array}{l}5 \\
5 \\
5 \\
5\end{array}$ \\
\hline \multirow[t]{5}{*}{$\begin{array}{l}\text { Polyester } \\
\text { Fiberglass } \\
\text { Board }\end{array}$} & $\begin{array}{c}\text { epoxy } 1 \\
\text { epoxy. } 2 \\
\text { polyurethane } \\
\text { acrylic }\end{array}$ & 0 & $\begin{array}{l}5 \\
5 \\
5 \\
5\end{array}$ & $\begin{array}{l}5 \\
5 \\
5 \\
5\end{array}$ & $\begin{array}{l}5 \\
5 \\
5 \\
5\end{array}$ & $\begin{array}{l}5 \\
5 \\
5 \\
5\end{array}$ & $\begin{array}{l}5 \\
5 \\
5 \\
5\end{array}$ & $\begin{array}{l}5 \\
5 \\
5 \\
5\end{array}$ \\
\hline & $\begin{array}{l}\text { epoxy } 1 \\
\text { epoxy } 2 \\
\text { polyurethane } \\
\text { acrylic }\end{array}$ & 7 & $\begin{array}{c}4 \\
5 \\
4-5 \\
5\end{array}$ & $\begin{array}{c}4 .-5 \\
5 \\
5 \\
5\end{array}$ & $\begin{array}{l}5 \\
5 \\
5 \\
5\end{array}$ & $\begin{array}{l}5 \\
5 \\
5 \\
5\end{array}$ & $\begin{array}{l}5 \\
5 \\
5 \\
5\end{array}$ & $\begin{array}{l}5 \\
5 \\
5 \\
5\end{array}$ \\
\hline & $\begin{array}{c}\text { epoxy } 1 \\
\text { epoxy } 2 \\
\text { polyurethane } \\
\text { acrylic }\end{array}$ & 14 & $\begin{array}{c}4 \\
4-5 \\
4-5 \\
4\end{array}$ & $\begin{array}{l}4-5 \\
4-5 \\
4-5 \\
4-5\end{array}$ & $\begin{array}{l}5 \\
5 \\
5 \\
5\end{array}$ & $\begin{array}{l}5 \\
5 \\
5 \\
5\end{array}$ & $\begin{array}{l}5 \\
5 \\
5 \\
5\end{array}$ & $\begin{array}{l}5 \\
5 \\
5 \\
5\end{array}$ \\
\hline & $\begin{array}{c}\text { epoxy } 1 \\
\text { epoxy } 2 \\
\text { polyurethane } \\
\text { acrylic }\end{array}$ & 21 & $\begin{array}{c}4 \\
4-5 \\
4-5 \\
4\end{array}$ & $\begin{array}{l}4-5 \\
4-5 \\
4-5 \\
4-5\end{array}$ & $\begin{array}{l}5 \\
5 \\
5 \\
5\end{array}$ & $\begin{array}{l}5 \\
5 \\
5 \\
5\end{array}$ & $\begin{array}{l}5 \\
5 \\
5 \\
5\end{array}$ & $\begin{array}{l}5 \\
5 \\
5 \\
5\end{array}$ \\
\hline & $\begin{array}{c}\text { epoxy } 1 \\
\text { epoxy } 2 \\
\text { polyurethane } \\
\text { acrylic }\end{array}$ & 28 & $\begin{array}{c}4 \\
4-5 \\
4 \\
4\end{array}$ & $\begin{array}{c}4 \\
4-5 \\
4-5 \\
4-5\end{array}$ & $\begin{array}{l}4 \\
5 \\
4 \\
4\end{array}$ & $\begin{array}{l}5 \\
5 \\
5 \\
5\end{array}$ & $\begin{array}{l}5 \\
5 \\
5 \\
5\end{array}$ & $\begin{array}{l}5 \\
5 \\
5 \\
5\end{array}$ \\
\hline
\end{tabular}

a See Table 3.8 for a description of the numerical ranking code for assessing tamper tapes. 
Table 4.8. (Continued)

\begin{tabular}{|c|c|c|c|c|c|c|c|c|}
\hline \multirow[b]{3}{*}{ Surface } & \multirow[b]{3}{*}{ Adhesive } & \multirow[b]{3}{*}{$\begin{array}{c}\text { Days of } \\
\text { Exposure }\end{array}$} & \multicolumn{6}{|c|}{ Condition of Tamper Tape ${ }^{a}$} \\
\hline & & & \multicolumn{2}{|c|}{ Appearance } & \multicolumn{2}{|c|}{ Security Emblem } & \multicolumn{2}{|c|}{ Surface Adhesion } \\
\hline & & & $\begin{array}{c}\text { Window } \\
\text { Area }\end{array}$ & $\begin{array}{l}\text { Vinyl } \\
\text { Area } \\
\end{array}$ & $\begin{array}{c}\text { Window } \\
\text { Area }\end{array}$ & $\begin{array}{l}\text { Vinyl } \\
\text { Area } \\
\end{array}$ & $\begin{array}{c}\text { Window } \\
\text { Area }\end{array}$ & Overall \\
\hline \multirow[t]{2}{*}{$\begin{array}{l}\text { Polyester } \\
\text { Fiberglass } \\
\text { Board } \\
\text { (Cont.) }\end{array}$} & $\begin{array}{c}\text { epoxy } 1 \\
\text { epoxy } 2 \\
\text { polyurethane } \\
\text { acrylic }\end{array}$ & 35 & $\begin{array}{c}4 \\
4-5 \\
4 \\
4\end{array}$ & $\begin{array}{l}3 \\
4-5 \\
4-5 \\
4-5\end{array}$ & $\begin{array}{l}4 \\
5 \\
4 \\
4\end{array}$ & $\begin{array}{l}5 \\
5 \\
5 \\
5\end{array}$ & $\begin{array}{l}5 \\
5 \\
5 \\
5\end{array}$ & $\begin{array}{l}5 \\
5 \\
5 \\
5\end{array}$ \\
\hline & $\begin{array}{c}\text { epoxy } 1 \\
\text { epoxy } 2 \\
\text { polyurethane } \\
\text { acrylic }\end{array}$ & 42 & $\begin{array}{c}3 \\
4-5 \\
3 \\
4\end{array}$ & $\begin{array}{l}3 \\
4-5 \\
4-5 \\
4\end{array}$ & $\begin{array}{l}4 \\
5 \\
4 \\
2\end{array}$ & $\begin{array}{l}5 \\
5 \\
5 \\
3\end{array}$ & $\begin{array}{l}5 \\
5 \\
5 \\
5\end{array}$ & $\begin{array}{l}5 \\
5 \\
5 \\
5\end{array}$ \\
\hline \multirow[t]{7}{*}{$\begin{array}{l}\text { Mil. Spec. } \\
\text { PUb } \\
\text { Painted } \\
\text { Steel }\end{array}$} & $\begin{array}{c}\text { epoxy } 1 \\
\text { epoxy } 2 \\
\text { polyurethane } \\
\text { acrylic }\end{array}$ & 0 & $\begin{array}{l}5 \\
5 \\
5 \\
5\end{array}$ & $\begin{array}{l}5 \\
5 \\
5 \\
5\end{array}$ & $\begin{array}{l}5 \\
5 \\
5 \\
5\end{array}$ & $\begin{array}{l}5 \\
5 \\
5 \\
5\end{array}$ & $\begin{array}{l}5 \\
5 \\
5 \\
5\end{array}$ & $\begin{array}{l}5 \\
5 \\
5 \\
5\end{array}$ \\
\hline & $\begin{array}{c}\text { epoxy } 1 \\
\text { epoxy } 2 \\
\text { polyurethane } \\
\text { acrylic }\end{array}$ & 7 & $\begin{array}{l}4-5 \\
5 \\
5 \\
4-5\end{array}$ & $\begin{array}{l}4-5 \\
5 \\
5 \\
4-5\end{array}$ & $\begin{array}{l}5 \\
5 \\
5 \\
5\end{array}$ & $\begin{array}{l}5 \\
5 \\
5 \\
5\end{array}$ & $\begin{array}{l}5 \\
5 \\
5 \\
5\end{array}$ & $\begin{array}{l}5 \\
5 \\
5 \\
5\end{array}$ \\
\hline & $\begin{array}{c}\text { epoxy } 1 \\
\text { epoxy } 2 \\
\text { polyurethane } \\
\text { acrylic }\end{array}$ & 14 & $\begin{array}{l}4 \\
4-5 \\
4-5 \\
4-5\end{array}$ & $\begin{array}{l}4 \\
4-5 \\
4-5 \\
4-5\end{array}$ & $\begin{array}{l}5 \\
5 \\
5 \\
5\end{array}$ & $\begin{array}{l}5 \\
5 \\
5 \\
5\end{array}$ & $\begin{array}{l}5 \\
5 \\
5 \\
5\end{array}$ & $\begin{array}{l}5 \\
5 \\
5 \\
5\end{array}$ \\
\hline & $\begin{array}{c}\text { epoxy } 1 \\
\text { epoxy } 2 \\
\text { polyurethane } \\
\text { acrylic }\end{array}$ & 21 & $\begin{array}{l}4 \\
4-5 \\
4-5 \\
4-5\end{array}$ & $\begin{array}{c}4 \\
4-5 \\
4-5 \\
4-5\end{array}$ & $\begin{array}{l}5 \\
5 \\
5 \\
5\end{array}$ & $\begin{array}{l}5 \\
5 \\
5 \\
5\end{array}$ & $\begin{array}{l}5 \\
5 \\
5 \\
5\end{array}$ & $\begin{array}{l}5 \\
5 \\
5 \\
5\end{array}$ \\
\hline & $\begin{array}{c}\text { epoxy } 1 \\
\text { epoxy } 2 \\
\text { polyurethane } \\
\text { acrylic }\end{array}$ & 28 & $\begin{array}{c}4 \\
4-5 \\
4 \\
4-5\end{array}$ & $\begin{array}{c}4 \\
4-5 \\
4-5 \\
4-5\end{array}$ & $\begin{array}{l}5 \\
5 \\
5 \\
4\end{array}$ & $\begin{array}{l}5 \\
5 \\
5 \\
5\end{array}$ & $\begin{array}{l}5 \\
5 \\
5 \\
5\end{array}$ & $\begin{array}{l}5 \\
5 \\
5 \\
5\end{array}$ \\
\hline & $\begin{array}{c}\text { epoxy } 1 \\
\text { epoxy } 2 \\
\text { polyurethane } \\
\text { acrylic }\end{array}$ & 35 & $\begin{array}{c}4 \\
4-5 \\
4 \\
4-5\end{array}$ & $\begin{array}{l}3 \\
4-5 \\
4-5 \\
4-5\end{array}$ & $\begin{array}{l}5 \\
5 \\
4 \\
4\end{array}$ & $\begin{array}{l}4 \\
5 \\
5 \\
4\end{array}$ & $\begin{array}{l}5 \\
5 \\
4 \\
5\end{array}$ & $\begin{array}{l}5 \\
5 \\
4 \\
5\end{array}$ \\
\hline & $\begin{array}{c}\text { epoxy } 1 \\
\text { epoxy } 2 \\
\text { polyurethane } \\
\text { acrylic }\end{array}$ & 42 & $\begin{array}{c}3 \\
4-5 \\
3 \\
4-5\end{array}$ & $\begin{array}{c}3 \\
4-5 \\
4 \\
4-5\end{array}$ & $\begin{array}{l}5 \\
5 \\
5 \\
3\end{array}$ & $\begin{array}{l}5 \\
5 \\
5 \\
4\end{array}$ & $\begin{array}{l}5 \\
5 \\
5 \\
5\end{array}$ & $\begin{array}{l}5 \\
5 \\
5 \\
5\end{array}$ \\
\hline
\end{tabular}

a See Table 3.8 for a description of the numerical ranking code for assessing tamper tapes.

$b$ Polyurethane 
materials, did not attack the latex Confirm ${ }^{\circledR}$ 1300. An examination of the tamper tapes' security features showed that the most loss in visibility occurred with the acrylic adhesive when tamper tapes were bonded to fiberglass board. Otherwise, there was only a slight or no change in visibility of the security features on tamper tapes bonded with the rapid-set adhesives. Adhesion, in general, was very good. The epoxy 2 adhesive essentially had no change in the 42 days of exposure.

\subsubsection{Thermal Cycling Exposure Results}

Tables 3.9, 3.10, and 3.11 give the results of the 42-day thermal cycling exposures for the tamper tapes made with Confirm ${ }^{\circledR} 1500$ without primer, Confirm ${ }^{\circledR} 1500$ with primer, and Confirm $^{\circledR} 1300$ materials, respectively. Changes in temperature from $-18^{\circ} \mathrm{C}$ to $46^{\circ} \mathrm{C}$ did not appear to affect the security feature or the adhesion of the tamper tapes nearly as much as did the conditions in the QUV cabinet. Overall, the tamper tapes held up very well throughout the thermal cycling exposure. The acrylic-adhesive bonded tamper tapes showed the same appearance and were attacked similarly as they were in the other exposures with this rapid-set adhesive, i.e., some of the security features on the tapes were essentially not visible or were very faded at the start of the exposure. Thermal cycling did not significantly worsen the condition of these tamper tapes. With the epoxy 1 and 2 adhesive-bonded tamper tapes, slight changes in the visibility of the security features were noted. With the epoxy 1 adhesive, these effects were apparent only on the tamper tapes prepared with the Confirm ${ }^{(B)} 1300$ and adhered to aluminum and steel surfaces. For the epoxy 2 adhesive, the effects were only on the tamper tapes prepared with the Confirm ${ }^{\circledR} 1500$ with primer material bonded to the fiberglass board. Otherwise, all of the other tamper tapes performed very well.

\subsection{SUMMARY}

Of the four Confirm ${ }^{\circledR}$ materials, i.e., 1700,1500 without primer, 1500 with primer, and 1300 , the Confirm ${ }^{\circledR} 1300$ was the least affected by the candidate rapid-set adhesives, especially the acrylic adhesive. The tamper tapes prepared with the Confirm ${ }^{\circledR} 1300$ also showed less deterioration in appearance and security feature visibility in comparison to tamper tapes prepared with the other Confirm ${ }^{\circledR}$ materials. Adhesion, in general, was quite good with all of the tamper tapes with one exception, that of the epoxy 1 on Lexan with the tamper tapes prepared from the Confirm 1700 material.

The epoxy 2 rapid-set adhesive weathered better than the other adhesives on tamper tapes prepared with any of the Confirm ${ }^{\circledR}$ materials. 
Table 4.9. Evaluation of Confirm ${ }^{\circledR} 1500$ Without Primer Tamper Tapes Applied with Candidate Rapid-Set Adhesives and Aged in the Thermal Cycling Cabinet $\left(-18^{\circ} \mathrm{C}\right.$ to $\left.46^{\circ} \mathrm{C}\right)$

\begin{tabular}{|c|c|c|c|c|c|c|c|c|}
\hline \multirow[b]{3}{*}{ Surface } & \multirow[b]{3}{*}{ Adhesive } & \multirow[b]{3}{*}{$\begin{array}{c}\text { Days of } \\
\text { Exposure }\end{array}$} & \multicolumn{6}{|c|}{ Condition of Tamper Tape ${ }^{a}$} \\
\hline & & & \multicolumn{2}{|c|}{ Appearance } & \multicolumn{2}{|c|}{ Security Emblem } & \multicolumn{2}{|c|}{ Surface Adhesion } \\
\hline & & & $\begin{array}{c}\text { Window } \\
\text { Area }\end{array}$ & $\begin{array}{l}\text { Vinyl } \\
\text { Area }\end{array}$ & $\begin{array}{c}\text { Window } \\
\text { Area }\end{array}$ & $\begin{array}{l}\text { Vinyl } \\
\text { Area }\end{array}$ & $\begin{array}{c}\text { Window } \\
\text { Area }\end{array}$ & Overall \\
\hline \multirow[t]{7}{*}{ Aluminum } & $\begin{array}{c}\text { epoxy } 1 \\
\text { epoxy } 2 \\
\text { polyurethane } \\
\text { acrylic }\end{array}$ & 0 & $\begin{array}{c}5 \\
4-5 \\
4-5 \\
2\end{array}$ & $\begin{array}{c}5 \\
4-5 \\
4-5 \\
2\end{array}$ & $\begin{array}{r}5 \\
.5 \\
5 \\
3\end{array}$ & $\begin{array}{l}5 \\
5 \\
5 \\
3\end{array}$ & $\begin{array}{l}5 \\
5 \\
5 \\
5\end{array}$ & $\begin{array}{l}5 \\
5 \\
5 \\
5\end{array}$ \\
\hline & $\begin{array}{c}\text { epoxy } 1 \\
\text { epoxy } 2 \\
\text { polyurethane } \\
\text { acrylic }\end{array}$ & 7 & $\begin{array}{c}5 \\
4 \\
4-5 \\
2\end{array}$ & $\begin{array}{c}5 \\
4 \\
4-5 \\
2\end{array}$ & $\begin{array}{l}5 \\
5 \\
5 \\
2\end{array}$ & $\begin{array}{l}5 \\
5 \\
5 \\
3\end{array}$ & $\begin{array}{l}5 \\
5 \\
5 \\
5\end{array}$ & $\begin{array}{l}5 \\
5 \\
5 \\
5\end{array}$ \\
\hline & $\begin{array}{c}\text { epoxy } 1 \\
\text { epoxy } 2 \\
\text { polyurethane } \\
\text { acrylic }\end{array}$ & 14 & $\begin{array}{c}4-5 \\
3-4 \\
4 \\
2\end{array}$ & $\begin{array}{c}4-5 \\
4 \\
4 \\
2\end{array}$ & $\begin{array}{l}5 \\
5 \\
5 \\
2\end{array}$ & $\begin{array}{l}5 \\
5 \\
5 \\
3\end{array}$ & $\begin{array}{l}5 \\
5 \\
5 \\
5\end{array}$ & $\begin{array}{l}5 \\
5 \\
5 \\
5\end{array}$ \\
\hline & $\begin{array}{c}\text { cpoxy } 1 \\
\text { epoxy } 2 \\
\text { polyurethane } \\
\text { acrylic }\end{array}$ & 21 & $\begin{array}{c}4-5 \\
3-4 \\
4 \\
2\end{array}$ & $\begin{array}{c}4-5 \\
4 \\
4 \\
2\end{array}$ & $\begin{array}{l}5 \\
5 \\
5 \\
2\end{array}$ & $\begin{array}{l}5 \\
5 \\
5 \\
3\end{array}$ & $\begin{array}{l}5 \\
5 \\
5 \\
5\end{array}$ & $\begin{array}{l}5 \\
5 \\
5 \\
5\end{array}$ \\
\hline & $\begin{array}{c}\text { epoxy } 1 \\
\text { epoxy } 2 \\
\text { polyurethane } \\
\text { acrylic }\end{array}$ & 28 & $\begin{array}{c}4-5 \\
3-4 \\
4 \\
1\end{array}$ & $\begin{array}{c}4-5 \\
4 \\
4 \\
2\end{array}$ & $\begin{array}{l}5 \\
5 \\
5 \\
2\end{array}$ & $\begin{array}{l}5 \\
5 \\
5 \\
3\end{array}$ & $\begin{array}{l}5 \\
5 \\
5 \\
2\end{array}$ & $\begin{array}{l}5 \\
5 \\
5 \\
3\end{array}$ \\
\hline & $\begin{array}{c}\text { epoxy } 1 \\
\text { epoxy } 2 \\
\text { polyurethane } \\
\text { acrylic }\end{array}$ & 35 & $\begin{array}{c}4-5 \\
3-4 \\
4 \\
1\end{array}$ & $\begin{array}{c}4-5 \\
4 \\
4 \\
2\end{array}$ & $\begin{array}{l}5 \\
5 \\
5 \\
2\end{array}$ & $\begin{array}{l}5 \\
5 \\
5 \\
2\end{array}$ & $\begin{array}{l}5 \\
5 \\
5 \\
2\end{array}$ & $\begin{array}{l}5 \\
5 \\
5 \\
3\end{array}$ \\
\hline & $\begin{array}{c}\text { epoxy } 1 \\
\text { epoxy } 2 \\
\text { polyurethanc } \\
\text { acrylic }\end{array}$ & 42 & $\begin{array}{c}4-5 \\
3-4 \\
4 \\
1\end{array}$ & $\begin{array}{c}4-5 \\
4 \\
4 \\
2\end{array}$ & $\begin{array}{l}5 \\
5 \\
5 \\
2\end{array}$ & $\begin{array}{l}5 \\
5 \\
5 \\
2\end{array}$ & $\begin{array}{l}5 \\
5 \\
5 \\
2\end{array}$ & $\begin{array}{l}5 \\
5 \\
5 \\
3\end{array}$ \\
\hline Siccl & $\begin{array}{c}\text { epoxy l } \\
\text { epoxy } 2 \\
\text { polyurethane } \\
\text { acrylic }\end{array}$ & 0 & $\begin{array}{c}5 \\
4-5 \\
4-5 \\
2\end{array}$ & $\begin{array}{c}5 \\
4-5 \\
4-5 \\
2\end{array}$ & $\begin{array}{l}5 \\
5 \\
5 \\
3\end{array}$ & $\begin{array}{l}5 \\
5 \\
5 \\
3\end{array}$ & $\begin{array}{l}5 \\
5 \\
5 \\
5\end{array}$ & $\begin{array}{l}5 \\
5 \\
5 \\
5\end{array}$ \\
\hline & $\begin{array}{c}\text { epoxy } 1 \\
\text { cpoxy } 2 \\
\text { polyurethane } \\
\text { acrylic }\end{array}$ & 7 & $\begin{array}{c}5 \\
4 \\
4-5 \\
2\end{array}$ & $\begin{array}{c}5 \\
4 \\
4-5 \\
2\end{array}$ & $\begin{array}{l}5 \\
5 \\
5 \\
2\end{array}$ & $\begin{array}{l}5 \\
5 \\
5 \\
3\end{array}$ & $\begin{array}{l}5 \\
5 \\
5 \\
5\end{array}$ & $\begin{array}{l}5 \\
5 \\
5 \\
5\end{array}$ \\
\hline
\end{tabular}

a See Table 3.8 for a description of the numerical ranking code for assessing tamper tapes. 
Table 4.9. (Continued)

\begin{tabular}{|c|c|c|c|c|c|c|c|c|}
\hline \multirow[b]{3}{*}{ Surface } & \multirow[b]{3}{*}{ Adhesive } & \multirow[b]{3}{*}{$\begin{array}{c}\text { Days of } \\
\text { Exposure }\end{array}$} & \multicolumn{6}{|c|}{ Condition of Tamper Tape ${ }^{a}$} \\
\hline & & & \multicolumn{2}{|c|}{ Appearance } & \multicolumn{2}{|c|}{ Security Emblem } & \multicolumn{2}{|c|}{ Surface Adhesion } \\
\hline & & & $\begin{array}{c}\text { Window } \\
\text { Area }\end{array}$ & $\begin{array}{l}\text { Vinyl } \\
\text { Area } \\
\end{array}$ & $\begin{array}{c}\text { Window } \\
\text { Area }\end{array}$ & $\begin{array}{l}\text { Vinyl } \\
\text { Area }\end{array}$ & $\begin{array}{c}\text { Window } \\
\text { Area }\end{array}$ & Overall \\
\hline $\begin{array}{l}\text { Steel } \\
\text { (Cont.) }\end{array}$ & $\begin{array}{c}\text { epoxy } 1 \\
\text { epoxy } 2 \\
\text { polyurethane } \\
\text { acrylic }\end{array}$ & 14 & $\begin{array}{c}4-5 \\
3-4 \\
4 \\
2\end{array}$ & $\begin{array}{c}4-5 \\
4 \\
4 \\
2\end{array}$ & $\begin{array}{l}5 \\
5 \\
5 \\
2\end{array}$ & $\begin{array}{l}5 \\
5 \\
5 \\
3\end{array}$ & $\begin{array}{l}5 \\
5 \\
5 \\
5\end{array}$ & $\begin{array}{l}5 \\
5 \\
5 \\
5\end{array}$ \\
\hline$\cdot$ & $\begin{array}{c}\text { epoxy } 1 \\
\text { epoxy } 2 \\
\text { polyurethane } \\
\text { acrylic }\end{array}$ & 21 & $\begin{array}{c}4-5 \\
3-4 \\
4 \\
2\end{array}$ & $\begin{array}{c}4-5 \\
4 \\
4 \\
2\end{array}$ & $\begin{array}{l}5 \\
5 \\
5 \\
2\end{array}$ & $\begin{array}{l}5 \\
5 \\
5 \\
3\end{array}$ & $\begin{array}{l}5 \\
5 \\
5 \\
5\end{array}$ & $\begin{array}{l}5 \\
5 \\
5 \\
5\end{array}$ \\
\hline & $\begin{array}{c}\text { epoxy } 1 \\
\text { epoxy } 2 \\
\text { polyurethane } \\
\text { acrylic }\end{array}$ & 28 & $\begin{array}{c}4-5 \\
3-4 \\
4 \\
2\end{array}$ & $\begin{array}{c}4-5 \\
4 \\
4 \\
2\end{array}$ & $\begin{array}{l}5 \\
5 \\
5 \\
2\end{array}$ & $\begin{array}{l}5 \\
5 \\
5 \\
3\end{array}$ & $\begin{array}{l}5 \\
5 \\
5 \\
2\end{array}$ & $\begin{array}{l}5 \\
5 \\
5 \\
3\end{array}$ \\
\hline & $\begin{array}{c}\text { epoxy } 1 \\
\text { epoxy } 2 \\
\text { polyurethane } \\
\text { acrylic }\end{array}$ & 35 & $\begin{array}{c}4-5 \\
3-4 \\
4 \\
2\end{array}$ & $\begin{array}{c}4-5 \\
4 \\
4 \\
2\end{array}$ & $\begin{array}{l}5 \\
5 \\
5 \\
2\end{array}$ & $\begin{array}{l}5 \\
5 \\
5 \\
3\end{array}$ & $\begin{array}{l}5 \\
5 \\
4 \\
2\end{array}$ & $\begin{array}{l}5 \\
5 \\
4 \\
3\end{array}$ \\
\hline & $\begin{array}{c}\text { epoxy } 1 \\
\text { epoxy } 2 \\
\text { polyurethane } \\
\text { acrylic }\end{array}$ & 42 & $\begin{array}{c}4-5 \\
3-4 \\
4 \\
2\end{array}$ & $\begin{array}{c}4-5 \\
4 \\
4 \\
2\end{array}$ & $\begin{array}{l}5 \\
5 \\
5 \\
2\end{array}$ & $\begin{array}{l}5 \\
5 \\
5 \\
3\end{array}$ & $\begin{array}{l}5 \\
5 \\
4 \\
2\end{array}$ & $\begin{array}{l}5 \\
5 \\
4 \\
3\end{array}$ \\
\hline \multirow[t]{5}{*}{$\begin{array}{l}\text { Polyester } \\
\text { Fiberglass } \\
\text { Board }\end{array}$} & $\begin{array}{c}\text { epoxy } 1 \\
\text { epoxy } 2 \\
\text { polyurethane } \\
\text { acrylic }\end{array}$ & 0 & $\begin{array}{l}5 \\
5 \\
5 \\
2\end{array}$ & $\begin{array}{l}5 \\
5 \\
5 \\
4\end{array}$ & $\begin{array}{l}5 \\
5 \\
5 \\
3\end{array}$ & $\begin{array}{l}5 \\
5 \\
5 \\
5\end{array}$ & $\begin{array}{l}5 \\
5 \\
5 \\
5\end{array}$ & $\begin{array}{l}5 \\
5 \\
5 \\
5\end{array}$ \\
\hline & $\begin{array}{c}\text { epoxy } 1 \\
\text { epoxy } 2 \\
\text { polyurethane } \\
\text { acrylic }\end{array}$ & 7 & $\begin{array}{c}5 \\
4-5 \\
5 \\
2\end{array}$ & $\begin{array}{c}5 \\
4-5 \\
5 \\
4\end{array}$ & $\begin{array}{r}5 \\
\cdot 5 \\
5 \\
3\end{array}$ & $\begin{array}{l}5 \\
5 \\
5 \\
5\end{array}$ & $\begin{array}{l}5 \\
5 \\
5 \\
5\end{array}$ & $\begin{array}{l}5 \\
5 \\
5 \\
5\end{array}$ \\
\hline & $\begin{array}{c}\text { epoxy } 1 \\
\text { epoxy } 2 \\
\text { polyurethane } \\
\text { acrylic }\end{array}$ & 14 & $\begin{array}{c}4-5 \\
4 \\
4-5 \\
2\end{array}$ & $\begin{array}{c}4-5 \\
4 \\
4-5 \\
4\end{array}$ & $\begin{array}{l}5 \\
5 \\
5 \\
3\end{array}$ & $\begin{array}{l}5 \\
5 \\
5 \\
5\end{array}$ & $\begin{array}{l}5 \\
5 \\
5 \\
5\end{array}$ & $\begin{array}{l}5 \\
5 \\
5 \\
5\end{array}$ \\
\hline & $\begin{array}{c}\text { cpoxy } 1 \\
\text { cpoxy } 2 \\
\text { polyurethane } \\
\text { acrylic }\end{array}$ & 21 & $\begin{array}{c}4-5 \\
4 \\
4-5 \\
2\end{array}$ & $\begin{array}{c}4-5 \\
4 \\
4-5 \\
4\end{array}$ & $\begin{array}{l}5 \\
5 \\
5 \\
3\end{array}$ & $\begin{array}{l}5 \\
5 \\
5 \\
5\end{array}$ & $\begin{array}{l}5 \\
5 \\
5 \\
5\end{array}$ & $\begin{array}{l}5 \\
5 \\
5 \\
5\end{array}$ \\
\hline & $\begin{array}{c}\text { epoxy } 1 \\
\text { epoxy } 2 \\
\text { polyurcthane } \\
\text { acrylic }\end{array}$ & 28 & $\begin{array}{c}4-5 \\
4 \\
4-5 \\
2\end{array}$ & $\begin{array}{c}4-5 \\
4 \\
4-5 \\
4\end{array}$ & $\begin{array}{l}5 \\
5 \\
5 \\
3\end{array}$ & $\begin{array}{l}5 \\
5 \\
5 \\
5\end{array}$ & $\begin{array}{l}5 \\
5 \\
5 \\
4\end{array}$ & $\begin{array}{l}5 \\
5 \\
5 \\
4\end{array}$ \\
\hline
\end{tabular}

a See Table 3.8 for a description of the numerical ranking code for assessing tamper tapes. 
Table 4.9. (Continued)

\begin{tabular}{|c|c|c|c|c|c|c|c|c|}
\hline \multirow[b]{3}{*}{ Surface } & \multirow[b]{3}{*}{ Adhesive } & \multirow[b]{3}{*}{$\begin{array}{l}\text { Days of } \\
\text { Exposure }\end{array}$} & \multicolumn{6}{|c|}{ Condition of Tamper Tape ${ }^{a}$} \\
\hline & & & \multicolumn{2}{|c|}{ Appearance } & \multicolumn{2}{|c|}{ Security Emblem } & \multirow{2}{*}{$\begin{array}{c}\text { Surface } \\
\text { Window } \\
\text { Area }\end{array}$} & \multirow{2}{*}{$\begin{array}{c}\text { Adhesion } \\
\text { 'Overal }\end{array}$} \\
\hline & & & $\begin{array}{c}\text { Window } \\
\text { Area }\end{array}$ & $\begin{array}{l}\text { Vinyl } \\
\text { Area }\end{array}$ & $\begin{array}{c}\text { Window } \\
\text { Area }\end{array}$ & $\begin{array}{l}\text { Vinyl } \\
\text { Area }\end{array}$ & & \\
\hline \multirow[t]{2}{*}{$\begin{array}{l}\text { Polyester } \\
\text { Fiberglass } \\
\text { Board } \\
\text { (Cont.) }\end{array}$} & $\begin{array}{l}\text { epoxy } 1 \\
\text { epoxy } 2 \\
\text { polyurethane } \\
\text { acrylic }\end{array}$ & 35 & $\begin{array}{c}4-5 \\
4 \\
45- \\
2\end{array}$ & $\begin{array}{c}4-5 \\
4 \\
4-5 \\
4\end{array}$ & $\begin{array}{l}5 \\
5 \\
5 \\
3\end{array}$ & $\begin{array}{l}5 \\
5 \\
5 \\
4\end{array}$ & $\begin{array}{l}5 \\
5 \\
5 \\
4\end{array}$ & $\begin{array}{l}5 \\
5 \\
5 \\
4\end{array}$ \\
\hline & $\begin{array}{l}\text { epoxy } 1 \\
\text { epoxy } 2 \\
\text { polyurethane } \\
\text { acrylic }\end{array}$ & 42 & $\begin{array}{c}4-5 \\
4 \\
4-5 \\
2\end{array}$ & $\begin{array}{c}4-5 \\
4 \\
4-5 \\
4\end{array}$ & $\begin{array}{l}5 \\
5 \\
5 \\
3\end{array}$ & $\begin{array}{l}5 \\
5 \\
5 \\
4\end{array}$ & $\begin{array}{l}5 \\
5 \\
5 \\
4\end{array}$ & $\begin{array}{l}5 \\
5 \\
5 \\
4\end{array}$ \\
\hline \multirow[t]{7}{*}{$\begin{array}{l}\text { Mil. Spec. } \\
\text { PUb } \\
\text { Painted } \\
\text { Steel }\end{array}$} & $\begin{array}{c}\text { epoxy } 1 \\
\text { epoxy } 2 \\
\text { polyurethane } \\
\text { acrylic }\end{array}$ & 0 & $\begin{array}{l}5 \\
5 \\
5 \\
2\end{array}$ & $\begin{array}{l}5 \\
5 \\
5 \\
3\end{array}$ & $\begin{array}{l}5 \\
5 \\
5 \\
2\end{array}$ & $\begin{array}{l}5 \\
5 \\
5 \\
3\end{array}$ & $\begin{array}{l}5 \\
5 \\
5 \\
5\end{array}$ & $\begin{array}{l}5 \\
5 \\
5 \\
5\end{array}$ \\
\hline & $\begin{array}{l}\text { epoxy } 1 \\
\text { epoxy } 2 \\
\text { polyurethane } \\
\text { acrylic }\end{array}$ & 7 & $\begin{array}{c}5 \\
4-5 \\
5 \\
2\end{array}$ & $\begin{array}{c}5 \\
4-5 \\
5 \\
3\end{array}$ & $\begin{array}{l}5 \\
5 \\
5 \\
2\end{array}$ & $\begin{array}{l}5 \\
5 \\
5 \\
3\end{array}$ & $\begin{array}{l}5 \\
5 \\
5 \\
5\end{array}$ & $\begin{array}{l}5 \\
5 \\
5 \\
5\end{array}$ \\
\hline & $\begin{array}{l}\text { epoxy } 1 \\
\text { epoxy } 2 \\
\text { polyurethane } \\
\text { acrylic }\end{array}$ & 14 & $\begin{array}{c}4-5 \\
4 \\
4-5 \\
2\end{array}$ & $\begin{array}{c}4-5 \\
4 \\
4-5 \\
3\end{array}$ & $\begin{array}{l}5 \\
5 \\
5 \\
2\end{array}$ & $\begin{array}{l}5 \\
5 \\
5 \\
3\end{array}$ & $\begin{array}{l}5 \\
5 \\
5 \\
5\end{array}$ & $\begin{array}{l}5 \\
5 \\
5 \\
5\end{array}$ \\
\hline & $\begin{array}{l}\text { epoxy } 1 \\
\text { epoxy } 2 \\
\text { polyurethane } \\
\text { acrylic }\end{array}$ & 21 & $\begin{array}{c}4-5 \\
4 \\
4-5 \\
2\end{array}$ & $\begin{array}{c}4-5 \\
4 \\
4-5 \\
3\end{array}$ & $\begin{array}{l}5 \\
5 \\
5 \\
2\end{array}$ & $\begin{array}{l}5 \\
5 \\
5 \\
3\end{array}$ & $\begin{array}{l}5 \\
5 \\
5 \\
5\end{array}$ & $\begin{array}{l}5 \\
5 \\
5 \\
5\end{array}$ \\
\hline & $\begin{array}{c}\text { epoxy } 1 \\
\text { epoxy } 2 \\
\text { polyurethane } \\
\text { acrylic }\end{array}$ & 28 & $\begin{array}{c}4-5 \\
4 \\
4-5 \\
2\end{array}$ & $\begin{array}{c}4-5 \\
4 \\
4-5 \\
3\end{array}$ & $\begin{array}{l}5 \\
5 \\
5 \\
2\end{array}$ & $\begin{array}{l}5 \\
5 \\
5 \\
3\end{array}$ & $\begin{array}{l}5 \\
5 \\
5 \\
3\end{array}$ & $\begin{array}{l}5 \\
5 \\
5 \\
4\end{array}$ \\
\hline & $\begin{array}{c}\text { epoxy } 1 \\
\text { epoxy } 2 \\
\text { polyurethane } \\
\text { acrylic }\end{array}$ & 35 & $\begin{array}{c}4-5 \\
4 \\
4 \\
2\end{array}$ & $\begin{array}{c}4-5 \\
4 \\
4-5 \\
3\end{array}$ & $\begin{array}{l}5 \\
5 \\
5 \\
2\end{array}$ & $\begin{array}{l}5 \\
5 \\
5 \\
3\end{array}$ & $\begin{array}{l}5 \\
5 \\
5 \\
3\end{array}$ & $\begin{array}{l}5 \\
5 \\
5 \\
3\end{array}$ \\
\hline & $\begin{array}{c}\text { epoxy } 1 \\
\text { epoxy } 2 \\
\text { polyurethane } \\
\text { acrylic }\end{array}$ & 42 & $\begin{array}{c}4-5 \\
4 \\
4 \\
2\end{array}$ & $\begin{array}{c}4-5 \\
4 \\
4-5 \\
3\end{array}$ & $\begin{array}{l}5 \\
5 \\
5 \\
2\end{array}$ & $\begin{array}{l}5 \\
5 \\
5 \\
3\end{array}$ & $\begin{array}{l}5 \\
5 \\
5 \\
2\end{array}$ & $\begin{array}{l}5 \\
5 \\
5 \\
3\end{array}$ \\
\hline
\end{tabular}

a See Table 3.8 for a description of the numerical ranking code for assessing tamper tapes.

$b$ Polyurethane 
Table 4.10. Evaluation of Confirm ${ }^{\circledR} 1500$ With Primer Tamper Tapes Applied with Candidate Rapid-Set Adhesives and Aged in the Thermal Cycling Cabinet $\left(-18^{\circ} \mathrm{C}\right.$ to $\left.46^{\circ} \mathrm{C}\right)$

\begin{tabular}{|c|c|c|c|c|c|c|c|c|}
\hline \multirow[b]{3}{*}{ Surface } & \multirow[b]{3}{*}{ Adhesive } & \multirow[b]{3}{*}{$\begin{array}{c}\text { Days of } \\
\text { Exposure }\end{array}$} & \multicolumn{6}{|c|}{ Condition of Tamper Tape ${ }^{a}$} \\
\hline & & & \multicolumn{2}{|c|}{ Appearance } & \multicolumn{2}{|c|}{ Security Emblem } & \multicolumn{2}{|c|}{ Surface Adhesion } \\
\hline & & & $\begin{array}{c}\text { Window } \\
\text { Area } \\
\end{array}$ & $\begin{array}{l}\text { Vinyl } \\
\text { Area } \\
\end{array}$ & $\begin{array}{c}\text { Window } \\
\text { Area }\end{array}$ & $\begin{array}{l}\text { Vinyl } \\
\text { Area } \\
\end{array}$ & $\begin{array}{c}\text { Window } \\
\text { Area } \\
\end{array}$ & Overall \\
\hline \multirow[t]{7}{*}{ Aluminum } & $\begin{array}{c}\text { epoxy } 1 \\
\text { epoxy } 2 \\
\text { polyurethane } \\
\text { acrylic }\end{array}$ & 0 & $\begin{array}{c}5 \\
5 \\
4-5 \\
4\end{array}$ & $\begin{array}{l}5 \\
5 \\
5 \\
5\end{array}$ & $\begin{array}{l}5 \\
5 \\
5 \\
5\end{array}$ & $\begin{array}{l}5 \\
5 \\
5 \\
5\end{array}$ & $\begin{array}{l}5 \\
5 \\
5 \\
5\end{array}$ & $\begin{array}{l}5 \\
5 \\
5 \\
5\end{array}$ \\
\hline & $\begin{array}{c}\text { epoxy } 1 \\
\text { epoxy } 2 \\
\text { polyurethane } \\
\text { acrylic }\end{array}$ & 7 & $\begin{array}{c}4 \\
5 \\
4-5 \\
4\end{array}$ & $\begin{array}{l}5 \\
5 \\
5 \\
5\end{array}$ & $\begin{array}{l}5 \\
5 \\
5 \\
5\end{array}$ & $\begin{array}{l}5 \\
5 \\
5 \\
5\end{array}$ & $\begin{array}{l}5 \\
5 \\
5 \\
5\end{array}$ & $\begin{array}{l}5 \\
5 \\
5 \\
5\end{array}$ \\
\hline & $\begin{array}{c}\text { epoxy } 1 \\
\text { epoxy } 2 \\
\text { polyurethane } \\
\text { acrylic }\end{array}$ & 14 & $\begin{array}{c}4 \\
4-5 \\
4 \\
4\end{array}$ & $\begin{array}{l}4-5 \\
4-5 \\
4 \\
5\end{array}$ & $\begin{array}{l}5 \\
5 \\
5 \\
5\end{array}$ & $\begin{array}{l}5 \\
5 \\
5 \\
5\end{array}$ & $\begin{array}{l}5 \\
5 \\
5 \\
5\end{array}$ & $\begin{array}{l}5 \\
5 \\
5 \\
5\end{array}$ \\
\hline & $\begin{array}{c}\text { epoxy } 1 \\
\text { epoxy } 2 \\
\text { polyurethane } \\
\text { acrylic }\end{array}$ & 21 & $\begin{array}{c}4 \\
4-5 \\
4 \\
4\end{array}$ & $\begin{array}{c}4-5 \\
4-5 \\
4 \\
5\end{array}$ & $\begin{array}{l}5 \\
5 \\
5 \\
4\end{array}$ & $\begin{array}{l}5 \\
5 \\
5 \\
5\end{array}$ & $\begin{array}{l}5 \\
5 \\
5 \\
5\end{array}$ & $\begin{array}{l}5 \\
5 \\
5 \\
5\end{array}$ \\
\hline & $\begin{array}{c}\text { epoxy } 1 \\
\text { epoxy } 2 \\
\text { polyurethane } \\
\text { acrylic }\end{array}$ & 28 & $\begin{array}{c}4 \\
4-5 \\
4 \\
4\end{array}$ & $\begin{array}{c}4-5 \\
4-5 \\
4 \\
5\end{array}$ & $\begin{array}{l}5 \\
5 \\
5 \\
4\end{array}$ & $\begin{array}{l}5 \\
5 \\
5 \\
5\end{array}$ & $\begin{array}{l}5 \\
5 \\
5 \\
5\end{array}$ & $\begin{array}{l}5 \\
5 \\
5 \\
5\end{array}$ \\
\hline & $\begin{array}{c}\text { epoxy } 1 \\
\text { epoxy } 2 \\
\text { polyurethane } \\
\text { acrylic }\end{array}$ & 35 & $\begin{array}{c}4 \\
4-5 \\
4 \\
4\end{array}$ & $\begin{array}{c}4-5 \\
4-5 \\
4 \\
5\end{array}$ & $\begin{array}{l}5 \\
5 \\
5 \\
3\end{array}$ & $\begin{array}{l}5 \\
5 \\
5 \\
4\end{array}$ & $\begin{array}{l}5 \\
5 \\
5 \\
5\end{array}$ & $\begin{array}{l}5 \\
5 \\
5 \\
5\end{array}$ \\
\hline & $\begin{array}{c}\text { epoxy } 1 \\
\text { epoxy } 2 \\
\text { polyurcthane } \\
\text { acrylic }\end{array}$ & 42 & $\begin{array}{c}4 \\
4-5 \\
4 \\
3\end{array}$ & $\begin{array}{c}4-5 \\
4-5 \\
4 \\
4\end{array}$ & $\begin{array}{l}5 \\
5 \\
5 \\
3\end{array}$ & $\begin{array}{l}5 \\
5 \\
5 \\
4\end{array}$ & $\begin{array}{l}5 \\
5 \\
5 \\
5\end{array}$ & $\begin{array}{l}5 \\
5 \\
5 \\
5\end{array}$ \\
\hline Steel & $\begin{array}{c}\text { epoxy } 1 \\
\text { epoxy } 2 \\
\text { polyurethane } \\
\text { acrylic }\end{array}$ & 0 & $\begin{array}{c}4-5 \\
5 \\
4-5 \\
4\end{array}$ & $\begin{array}{l}5 \\
5 \\
5 \\
5\end{array}$ & $\begin{array}{l}5 \\
5 \\
5 \\
5\end{array}$ & $\begin{array}{l}5 \\
5 \\
5 \\
5\end{array}$ & $\begin{array}{l}5 \\
5 \\
5 \\
5\end{array}$ & $\begin{array}{l}5 \\
5 \\
5 \\
5\end{array}$ \\
\hline & $\begin{array}{c}\text { epoxy } 1 \\
\text { epoxy } 2 \\
\text { polyurethane } \\
\text { acrylic }\end{array}$ & 7 & $\begin{array}{c}4 \\
5 \\
4-5 \\
4\end{array}$ & $\begin{array}{l}5 \\
5 \\
5 \\
5\end{array}$ & $\begin{array}{l}5 \\
5 \\
5 \\
4\end{array}$ & $\begin{array}{l}5 \\
5 \\
5 \\
5\end{array}$ & $\begin{array}{l}5 \\
5 \\
5 \\
5\end{array}$ & $\begin{array}{l}5 \\
5 \\
5 \\
5\end{array}$ \\
\hline
\end{tabular}

a See Table 3.8 for a description of the numerical ranking code for assessing tamper tapes. 
Table 4.10. (Continued)

\begin{tabular}{|c|c|c|c|c|c|c|c|c|}
\hline \multirow[b]{3}{*}{ Surface } & \multirow[b]{3}{*}{ Adhesive } & \multirow[b]{3}{*}{$\begin{array}{c}\text { Days of } \\
\text { Exposure }\end{array}$} & \multicolumn{6}{|c|}{ Condition of Tamper Tape ${ }^{a}$} \\
\hline & & & \multicolumn{2}{|c|}{ Appearance } & \multicolumn{2}{|c|}{ Security Emblem } & \multicolumn{2}{|c|}{ Surface Adhesion } \\
\hline & & & $\begin{array}{c}\text { Window } \\
\text { Area }\end{array}$ & $\begin{array}{l}\text { Vinyl } \\
\text { Area }\end{array}$ & $\begin{array}{c}\text { Window } \\
\text { Area }\end{array}$ & $\begin{array}{l}\text { Vinyl } \\
\text { Area }\end{array}$ & $\begin{array}{c}\text { Window } \\
\text { Area }\end{array}$ & Overall \\
\hline \multirow[t]{5}{*}{$\begin{array}{l}\text { Steel } \\
\text { (Cont.) }\end{array}$} & $\begin{array}{c}\text { epoxy } 1 \\
\text { epoxy } 2 \\
\text { polyurethane } \\
\text { acrylic }\end{array}$ & 14 & $\begin{array}{c}3-4 \\
4-5 \\
4 \\
4\end{array}$ & $\begin{array}{l}4-5 \\
4-5 \\
4-5 \\
5\end{array}$ & $\begin{array}{l}5 \\
5 \\
5 \\
4\end{array}$ & $\begin{array}{l}5 \\
5 \\
5 \\
5\end{array}$ & $\begin{array}{l}5 \\
5 \\
5 \\
5\end{array}$ & $\begin{array}{l}5 \\
5 \\
5 \\
5\end{array}$ \\
\hline & $\begin{array}{c}\text { epoxy } 1 \\
\text { epoxy } 2 \\
\text { polyurethane } \\
\text { acrylic }\end{array}$ & 21 & $\begin{array}{c}3-4 \\
4-5 \\
4 \\
4\end{array}$ & $\begin{array}{c}4-5 \\
4-5 \\
4-5 \\
5\end{array}$ & $\begin{array}{l}5 \\
5 \\
5 \\
4\end{array}$ & $\begin{array}{l}5 \\
5 \\
5 \\
5\end{array}$ & $\begin{array}{l}5 \\
5 \\
5 \\
5\end{array}$ & $\begin{array}{l}5 \\
5 \\
5 \\
5\end{array}$ \\
\hline & $\begin{array}{c}\text { epoxy } 1 \\
\text { epoxy } 2 \\
\text { polyurethane } \\
\text { acrylic }\end{array}$ & 28 & $\begin{array}{c}3-4 \\
4-5 \\
4 \\
4\end{array}$ & $\begin{array}{c}4-5 \\
4-5 \\
4-5 \\
5\end{array}$ & $\begin{array}{l}5 \\
5 \\
5 \\
3\end{array}$ & $\begin{array}{l}5 \\
5 \\
5 \\
5\end{array}$ & $\begin{array}{l}5 \\
5 \\
5 \\
5\end{array}$ & $\begin{array}{l}5 \\
5 \\
5 \\
5\end{array}$ \\
\hline & $\begin{array}{c}\text { epoxy } 1 \\
\text { epoxy } 2 \\
\text { polyurethane } \\
\text { acrylic }\end{array}$ & 35 & $\begin{array}{c}3-4 \\
4-5 \\
4 \\
4\end{array}$ & $\begin{array}{l}4-5 \\
4-5 \\
4-5 \\
4-5\end{array}$ & $\begin{array}{l}5 \\
5 \\
5 \\
3\end{array}$ & $\begin{array}{l}5 \\
5 \\
5 \\
5\end{array}$ & $\begin{array}{l}5 \\
5 \\
5 \\
5\end{array}$ & $\begin{array}{l}5 \\
5 \\
5 \\
5\end{array}$ \\
\hline & $\begin{array}{c}\text { epoxy } 1 \\
\text { epoxy } 2 \\
\text { polyurethane } \\
\text { acrylic }\end{array}$ & 42 & $\begin{array}{c}3-4 \\
4-5 \\
4 \\
4\end{array}$ & $\begin{array}{l}4-5 \\
4-5 \\
4-5 \\
4-5\end{array}$ & $\begin{array}{l}5 \\
5 \\
5 \\
3\end{array}$ & $\begin{array}{l}5 \\
5 \\
5 \\
4\end{array}$ & $\begin{array}{l}5 \\
5 \\
5 \\
5\end{array}$ & $\begin{array}{l}5 \\
5 \\
5 \\
5\end{array}$ \\
\hline \multirow[t]{5}{*}{$\begin{array}{l}\text { Polyester } \\
\text { Fiberglass } \\
\text { Board }\end{array}$} & $\begin{array}{l}\text { epoxy } 1 \\
\text { epoxy } 2 \\
\text { polyurethane } \\
\text { acrylic }\end{array}$ & 0 & $\begin{array}{l}5 \\
5 \\
5 \\
2\end{array}$ & $\begin{array}{l}5 \\
5 \\
5 \\
2\end{array}$ & $\begin{array}{l}5 \\
5 \\
5 \\
3\end{array}$ & $\begin{array}{l}5 \\
5 \\
5 \\
5\end{array}$ & $\begin{array}{l}5 \\
5 \\
5 \\
5\end{array}$ & $\begin{array}{l}5 \\
5 \\
5 \\
5\end{array}$ \\
\hline & $\begin{array}{l}\text { epoxy } 1 \\
\text { epoxy } 2 \\
\text { polyurethane } \\
\text { acrylic }\end{array}$ & 7 & $\begin{array}{c}4-5 \\
5 \\
5 \\
2\end{array}$ & $\begin{array}{l}5 \\
5 \\
5 \\
2\end{array}$ & $\begin{array}{l}5 \\
5 \\
5 \\
2\end{array}$ & $\begin{array}{l}5 \\
5 \\
5 \\
5\end{array}$ & $\begin{array}{l}5 \\
5 \\
5 \\
5\end{array}$ & $\begin{array}{l}5 \\
5 \\
5 \\
5\end{array}$ \\
\hline & $\begin{array}{c}\text { epoxy } 1 \\
\text { epoxy } 2 \\
\text { polyurethane } \\
\text { acrylic }\end{array}$ & 14 & $\begin{array}{c}4 \\
4-5 \\
4-5 \\
2\end{array}$ & $\begin{array}{c}4-5 \\
4-5 \\
4-5 \\
2\end{array}$ & $\begin{array}{l}5 \\
5 \\
5 \\
2\end{array}$ & $\begin{array}{l}5 \\
5 \\
5 \\
5\end{array}$ & $\begin{array}{l}5 \\
5 \\
5 \\
5\end{array}$ & $\begin{array}{l}5 \\
5 \\
5 \\
5\end{array}$ \\
\hline & $\begin{array}{c}\text { epoxy } 1 \\
\text { epoxy } 2 \\
\text { polyurethane } \\
\text { acrylic }\end{array}$ & 21 & $\begin{array}{c}4 \\
4-5 \\
4-5 \\
2\end{array}$ & $\begin{array}{l}4-5 \\
4-5 \\
4-5 \\
2\end{array}$ & $\begin{array}{l}5 \\
5 \\
5 \\
2\end{array}$ & $\begin{array}{l}5 \\
5 \\
5 \\
5\end{array}$ & $\begin{array}{l}5 \\
5 \\
5 \\
5\end{array}$ & $\begin{array}{l}5 \\
5 \\
5 \\
5\end{array}$ \\
\hline & $\begin{array}{c}\text { epoxy } 1 \\
\text { epoxy } 2 \\
\text { polyurethane } \\
\text { acrylic }\end{array}$ & 28 & $\begin{array}{c}4 \\
4-5 \\
4-5 \\
2\end{array}$ & $\begin{array}{l}4-5 \\
4-5 \\
4-5 \\
2\end{array}$ & $\begin{array}{l}5 \\
5 \\
5 \\
2\end{array}$ & $\begin{array}{l}5 \\
5 \\
5 \\
4\end{array}$ & $\begin{array}{l}5 \\
5 \\
5 \\
3\end{array}$ & $\begin{array}{l}5 \\
5 \\
5 \\
3\end{array}$ \\
\hline
\end{tabular}

a See Table 3.8 for a description of the numerical ranking code for assessing tamper tapes. 
Table 4.10. (Continued)

\begin{tabular}{|c|c|c|c|c|c|c|c|c|}
\hline \multirow[t]{2}{*}{ - } & & & \multicolumn{6}{|c|}{ Condition of Tamper Tape ${ }^{a}$} \\
\hline & & & \multicolumn{2}{|c|}{ Appearance } & \multirow{2}{*}{$\begin{array}{c}\text { Security } \\
\text { Window } \\
\text { Area } \\
\end{array}$} & \multirow{2}{*}{$\begin{array}{c}\text { Emblem } \\
\text { Vinyl } \\
\text { Area } \\
\end{array}$} & \multirow{2}{*}{$\begin{array}{c}\text { Surface } \\
\text { Window } \\
\text { Area }\end{array}$} & \multirow{2}{*}{$\begin{array}{r}\text { Adhesion } \\
\text { Overall } \\
\end{array}$} \\
\hline Surface & Adhesive & $\begin{array}{c}\text { Days of } \\
\text { Exposure } \\
\end{array}$ & $\begin{array}{c}\text { Window } \\
\text { Area }\end{array}$ & $\begin{array}{l}\text { Vinyl } \\
\text { Area } \\
\end{array}$ & & & & \\
\hline \multirow[t]{2}{*}{$\begin{array}{l}\text { Polyester } \\
\text { Fiberglass } \\
\text { Board } \\
\text { (Cont.) }\end{array}$} & $\begin{array}{c}\text { epoxy } 1 \\
\text { epoxy } 2 \\
\text { polyurethane } \\
\text { acrylic }\end{array}$ & 35 & $\begin{array}{c}4 \\
4-5 \\
4 \\
2\end{array}$ & $\begin{array}{c}4-5 \\
4-5 \\
4-5 \\
2\end{array}$ & $\begin{array}{l}5 \\
5 \\
5 \\
2\end{array}$ & $\begin{array}{l}5 \\
5 \\
5 \\
4\end{array}$ & $\begin{array}{l}5 \\
5 \\
5 \\
3\end{array}$ & $\begin{array}{l}5 \\
5 \\
5 \\
3\end{array}$ \\
\hline & $\begin{array}{c}\text { epoxy } 1 \\
\text { epoxy } 2 \\
\text { polyurethane } \\
\text { acrylic }\end{array}$ & 42 & $\begin{array}{l}4 \\
4-5 \\
4 \\
2\end{array}$ & $\begin{array}{c}4-5 \\
4-5 \\
4-5 \\
2\end{array}$ & $\begin{array}{l}5 \\
5 \\
5 \\
2\end{array}$ & $\begin{array}{l}5 \\
5 \\
5 \\
3\end{array}$ & $\begin{array}{l}5 \\
5 \\
5 \\
3\end{array}$ & $\begin{array}{l}5 \\
5 \\
5 \\
3\end{array}$ \\
\hline \multirow[t]{7}{*}{$\begin{array}{l}\text { Mil. Spec. } \\
\text { PUb } \\
\text { Painted } \\
\text { Steel }\end{array}$} & $\begin{array}{c}\text { epoxy } 1 \\
\text { epoxy } 2 \\
\text { polyurethane } \\
\text { acrylic }\end{array}$ & 0 & $\begin{array}{c}4-5 \\
5 \\
5 \\
2\end{array}$ & $\begin{array}{l}5 \\
5 \\
5 \\
2\end{array}$ & $\begin{array}{l}5 \\
5 \\
5 \\
2\end{array}$ & $\begin{array}{l}5 \\
5 \\
5 \\
4\end{array}$ & $\begin{array}{l}5 \\
5 \\
5 \\
5\end{array}$ & $\begin{array}{l}5 \\
5 \\
5 \\
5\end{array}$ \\
\hline & $\begin{array}{c}\text { epoxy } 1 \\
\text { epoxy } 2 \\
\text { polyurethane } \\
\text { acrylic }\end{array}$ & 7 & $\begin{array}{l}4-5 \\
5 \\
5 \\
2\end{array}$ & $\begin{array}{l}5 \\
5 \\
5 \\
2\end{array}$ & $\begin{array}{l}5 \\
5 \\
5 \\
2\end{array}$ & $\begin{array}{l}5 \\
5 \\
5 \\
4\end{array}$ & $\begin{array}{l}5 \\
5 \\
5 \\
5\end{array}$ & $\begin{array}{l}5 \\
5 \\
5 \\
5\end{array}$ \\
\hline & $\begin{array}{c}\text { epoxy } 1 \\
\text { epoxy } 2 \\
\text { polyurethane } \\
\text { acrylic }\end{array}$ & 14 & $\begin{array}{c}4 \\
4-5 \\
4-5 \\
2\end{array}$ & $\begin{array}{c}4-5 \\
4-5 \\
4-5 \\
2\end{array}$ & $\begin{array}{l}5 \\
5 \\
5 \\
2\end{array}$ & $\begin{array}{l}5 \\
5 \\
5 \\
4\end{array}$ & $\begin{array}{l}5 \\
5 \\
5 \\
5\end{array}$ & $\begin{array}{l}5 \\
5 \\
5 \\
5\end{array}$ \\
\hline & $\begin{array}{c}\text { epoxy } 1 \\
\text { epoxy } 2 \\
\text { polyurethane } \\
\text { acrylic }\end{array}$ & 21 & $\begin{array}{c}4 \\
4-5 \\
4-5 \\
2\end{array}$ & $\begin{array}{c}4-5 \\
4-5 \\
4-5 \\
2\end{array}$ & $\begin{array}{l}5 \\
5 \\
5 \\
2\end{array}$ & $\begin{array}{l}5 \\
5 \\
5 \\
4\end{array}$ & $\begin{array}{l}5 \\
5 \\
5 \\
5\end{array}$ & $\begin{array}{l}5 \\
5 \\
5 \\
5\end{array}$ \\
\hline & $\begin{array}{c}\text { epoxy } 1 \\
\text { epoxy } 2 \\
\text { polyurethane } \\
\text { acrylic }\end{array}$ & 28 & $\begin{array}{c}4 \\
4-5 \\
4-5 \\
2\end{array}$ & $\begin{array}{c}4-5 \\
4-5 \\
4-5 \\
2\end{array}$ & $\begin{array}{l}5 \\
5 \\
5 \\
2\end{array}$ & $\begin{array}{l}5 \\
5 \\
5 \\
4\end{array}$ & $\begin{array}{l}5 \\
5 \\
5 \\
4\end{array}$ & $\begin{array}{l}5 \\
5 \\
5 \\
4\end{array}$ \\
\hline & $\begin{array}{c}\text { epoxy } 1 \\
\text { epoxy } 2 \\
\text { polyurethane } \\
\text { acrylic }\end{array}$ & 35 & $\begin{array}{c}4 \\
4-5 \\
4 \\
2\end{array}$ & $\begin{array}{c}4-5 \\
4-5 \\
4-5 \\
2\end{array}$ & $\begin{array}{l}5 \\
5 \\
5 \\
2\end{array}$ & $\begin{array}{l}5 \\
5 \\
5 \\
4\end{array}$ & $\begin{array}{l}5 \\
5 \\
5 \\
4\end{array}$ & $\begin{array}{l}5 \\
5 \\
5 \\
4\end{array}$ \\
\hline & $\begin{array}{c}\text { epoxy } 1 \\
\text { epoxy } 2 \\
\text { polyurethane } \\
\text { acrylic }\end{array}$ & 42 & $\begin{array}{c}4 \\
4-5 \\
4 \\
2\end{array}$ & $\begin{array}{c}4-5 \\
4-5 \\
4-5 \\
2\end{array}$ & $\begin{array}{l}5 \\
5 \\
5 \\
2\end{array}$ & $\begin{array}{l}5 \\
5 \\
5 \\
3\end{array}$ & $\begin{array}{l}5 \\
5 \\
5 \\
4\end{array}$ & $\begin{array}{l}5 \\
5 \\
5 \\
4\end{array}$ \\
\hline
\end{tabular}

a See Table 3.8 for a description of the numerical ranking code for assessing tamper tapes.

$b$ Polyurethane 
Table 4.11. Evaluation of Confirm ${ }^{\circledR} 1300$ Tamper Tapes Applied with Candidate Rapid-Set Adhesives and Aged in the Thermal Cycling Cabinet $\left(-18^{\circ} \mathrm{C}\right.$ to $\left.46^{\circ} \mathrm{C}\right)$

\begin{tabular}{|c|c|c|c|c|c|c|c|c|}
\hline \multirow[b]{3}{*}{ Surface } & \multirow[b]{3}{*}{ Adhesive } & \multirow[b]{3}{*}{$\begin{array}{c}\text { Days of } \\
\text { Exposure }\end{array}$} & \multicolumn{6}{|c|}{ Condition of Tamper Tape ${ }^{a}$} \\
\hline & & & \multicolumn{2}{|c|}{ Appearance } & \multicolumn{2}{|c|}{ Security Emblem } & \multicolumn{2}{|c|}{ Surface Adhesion } \\
\hline & & & $\begin{array}{c}\text { Window } \\
\text { Area }\end{array}$ & $\begin{array}{l}\text { Vinyl } \\
\text { Area }\end{array}$ & $\begin{array}{c}\text { Window } \\
\text { Area }\end{array}$ & $\begin{array}{l}\text { Vinyl } \\
\text { Area }\end{array}$ & $\begin{array}{c}\text { Window } \\
\text { Area }\end{array}$ & Overall \\
\hline \multirow[t]{7}{*}{ Aluminum } & $\begin{array}{c}\text { epoxy } 1 \\
\text { epoxy } 2 \\
\text { polyurethane } \\
\text { acrylic }\end{array}$ & 0 & $\begin{array}{l}5 \\
5 \\
5 \\
5\end{array}$ & $\begin{array}{l}5 \\
5 \\
5 \\
5\end{array}$ & $\begin{array}{l}5 \\
5 \\
5 \\
5\end{array}$ & $\begin{array}{l}5 \\
5 \\
5 \\
5\end{array}$ & $\begin{array}{l}5 \\
5 \\
5 \\
5\end{array}$ & $\begin{array}{l}5 \\
5 \\
5 \\
5\end{array}$ \\
\hline & $\begin{array}{c}\text { epoxy } 1 \\
\text { epoxy } 2 \\
\text { polyurethane } \\
\text { acrylic }\end{array}$ & 7 & $\begin{array}{l}5 \\
5 \\
5 \\
5\end{array}$ & $\begin{array}{l}5 \\
5 \\
5 \\
5\end{array}$ & $\begin{array}{l}5 \\
5 \\
5 \\
5\end{array}$ & $\begin{array}{l}5 \\
5 \\
5 \\
5\end{array}$ & $\begin{array}{l}5 \\
5 \\
5 \\
5\end{array}$ & $\begin{array}{l}5 \\
5 \\
5 \\
5\end{array}$ \\
\hline & $\begin{array}{l}\text { epoxy } 1 \\
\text { epoxy } 2 \\
\text { polyurethane } \\
\text { acrylic }\end{array}$ & 14 & $\begin{array}{l}4-5 \\
4-5 \\
4-5 \\
4-5\end{array}$ & $\begin{array}{l}4-5 \\
4-5 \\
4-5 \\
4-5\end{array}$ & $\begin{array}{l}5 \\
5 \\
5 \\
5\end{array}$ & $\begin{array}{l}5 \\
5 \\
5 \\
5\end{array}$ & $\begin{array}{l}5 \\
5 \\
5 \\
5\end{array}$ & $\begin{array}{l}5 \\
5 \\
5 \\
5\end{array}$ \\
\hline & $\begin{array}{c}\text { epoxy } 1 \\
\text { epoxy } 2 \\
\text { polyurethane } \\
\text { acrylic }\end{array}$ & 21 & $\begin{array}{l}4-5 \\
4-5 \\
4-5 \\
4-5\end{array}$ & $\begin{array}{l}4-5 \\
4-5 \\
4-5 \\
4-5\end{array}$ & $\begin{array}{l}5 \\
5 \\
5 \\
5\end{array}$ & $\begin{array}{l}5 \\
5 \\
5 \\
5\end{array}$ & $\begin{array}{l}5 \\
5 \\
5 \\
5\end{array}$ & $\begin{array}{l}5 \\
5 \\
5 \\
5\end{array}$ \\
\hline & $\begin{array}{c}\text { epoxy } 1 \\
\text { epoxy } 2 \\
\text { polyurethane } \\
\text { acrylic }\end{array}$ & 28 & $\begin{array}{l}4-5 \\
4-5 \\
4-5 \\
4-5\end{array}$ & $\begin{array}{l}4-5 \\
4-5 \\
4-5 \\
4-5\end{array}$ & $\begin{array}{l}5 \\
5 \\
5 \\
5\end{array}$ & $\begin{array}{l}5 \\
5 \\
5 \\
5\end{array}$ & $\begin{array}{l}5 \\
5 \\
5 \\
5\end{array}$ & $\begin{array}{l}5 \\
5 \\
5 \\
5\end{array}$ \\
\hline & $\begin{array}{c}\text { epoxy } 1 \\
\text { epoxy } 2 \\
\text { polyurethane } \\
\text { acrylic }\end{array}$ & 35 & $\begin{array}{l}4-5 \\
4-5 \\
4-5 \\
4-5\end{array}$ & $\begin{array}{l}4-5 \\
4-5 \\
4-5 \\
4-5\end{array}$ & $\begin{array}{l}4 \\
5 \\
5 \\
5\end{array}$ & $\begin{array}{r}5 \\
5 \\
.5 \\
5\end{array}$ & $\begin{array}{l}5 \\
5 \\
5 \\
5\end{array}$ & $\begin{array}{l}5 \\
5 \\
5 \\
5\end{array}$ \\
\hline & $\begin{array}{l}\text { epoxy } 1 \\
\text { epoxy } 2 \\
\text { polyurethane } \\
\text { acrylic }\end{array}$ & 42 & $\begin{array}{l}4-5 \\
4-5 \\
4-5 \\
4-5\end{array}$ & $\begin{array}{l}4-5 \\
4-5 \\
4-5 \\
4-5\end{array}$ & $\begin{array}{l}4 \\
5 \\
5 \\
5\end{array}$ & $\begin{array}{l}5 \\
5 \\
5 \\
5\end{array}$ & $\begin{array}{l}5 \\
5 \\
5 \\
5\end{array}$ & $\begin{array}{l}5 \\
5 \\
5 \\
5\end{array}$ \\
\hline Steel & $\begin{array}{c}\text { epoxy } 1 \\
\text { epoxy } 2 \\
\text { polyurethane } \\
\text { acrylic }\end{array}$ & 0 & $\begin{array}{c}5 \\
4-5 \\
5 \\
5\end{array}$ & $\begin{array}{c}5 \\
4-5 \\
5 \\
5\end{array}$ & $\begin{array}{l}5 \\
5 \\
5 \\
5\end{array}$ & $\begin{array}{l}5 \\
5 \\
5 \\
5\end{array}$ & $\begin{array}{l}5 \\
5 \\
5 \\
5\end{array}$ & $\begin{array}{l}5 \\
5 \\
5 \\
5\end{array}$ \\
\hline & $\begin{array}{c}\text { epoxy } 1 \\
\text { cpoxy } 2 \\
\text { polyurethane } \\
\text { acrylic }\end{array}$ & 7 & $\begin{array}{c}5 \\
4-5 \\
5 \\
5\end{array}$ & $\begin{array}{c}5 \\
4-5 \\
5 \\
5\end{array}$ & $\begin{array}{l}5 \\
5 \\
5 \\
5\end{array}$ & $\begin{array}{l}5 \\
5 \\
5 \\
5\end{array}$ & $\begin{array}{l}5 \\
5 \\
5 \\
5\end{array}$ & $\begin{array}{l}5 \\
5 \\
5 \\
5\end{array}$ \\
\hline
\end{tabular}

a See Table 3.8 for a description of the numerical ranking code for assessing tamper tapes. 
Table 4.11. (Continued)

\begin{tabular}{|c|c|c|c|c|c|c|c|c|}
\hline \multirow[b]{3}{*}{ Surface } & \multirow[b]{3}{*}{ Adhesive } & \multirow[b]{3}{*}{$\begin{array}{c}\text { Days of } \\
\text { Exposure }\end{array}$} & \multicolumn{6}{|c|}{ Condition of Tamper Tape ${ }^{a}$} \\
\hline & & & \multicolumn{2}{|c|}{ Appearance } & \multicolumn{2}{|c|}{ Security Emblem } & \multicolumn{2}{|c|}{ Surface Adhesion } \\
\hline & & & $\begin{array}{c}\text { Window } \\
\text { Area }\end{array}$ & $\begin{array}{l}\text { Vinyl } \\
\text { Area }\end{array}$ & $\begin{array}{c}\text { Window } \\
\text { Area }\end{array}$ & $\begin{array}{c}\text { Vinyl } \\
\text { Area }\end{array}$ & $\begin{array}{c}\text { Window } \\
\text { Area }\end{array}$ & Overall \\
\hline \multirow[t]{5}{*}{$\begin{array}{l}\text { Steel } \\
\text { (Cont.) }\end{array}$} & $\begin{array}{c}\text { epoxy } 1 \\
\text { epoxy } 2 \\
\text { polyurethane } \\
\text { acrylic }\end{array}$ & 14 & $\begin{array}{c}4-5 \\
4 \\
4-5 \\
4-5\end{array}$ & $\begin{array}{c}4-5 \\
4 \\
4-5 \\
4\end{array}$ & $\begin{array}{l}5 \\
5 \\
5 \\
5\end{array}$ & $\begin{array}{l}5 \\
5 \\
5 \\
5\end{array}$ & $\begin{array}{l}5 \\
5 \\
5 \\
5\end{array}$ & $\begin{array}{l}5 \\
5 \\
5 \\
5\end{array}$ \\
\hline & $\begin{array}{c}\text { epoxy } 1 \\
\text { epoxy } 2 \\
\text { polyurethane } \\
\text { acrylic }\end{array}$ & 21 & $\begin{array}{c}4-5 \\
4 \\
4-5 \\
4-5\end{array}$ & $\begin{array}{c}4-5 \\
4 \\
4-5 \\
4\end{array}$ & $\begin{array}{l}5 \\
5 \\
5 \\
5\end{array}$ & $\begin{array}{l}5 \\
5 \\
5 \\
5\end{array}$ & $\begin{array}{l}5 \\
5 \\
5 \\
5\end{array}$ & $\begin{array}{l}5 \\
5 \\
5 \\
5\end{array}$ \\
\hline & $\begin{array}{c}\text { epoxy } 1 \\
\text { epoxy } 2 \\
\text { polyurethane } \\
\text { acrylic }\end{array}$ & 28 & $\begin{array}{c}4-5 \\
4 \\
4-5 \\
4-5\end{array}$ & $\begin{array}{c}4-5 \\
4 \\
4-5 \\
4\end{array}$ & $\begin{array}{l}5 \\
5 \\
5 \\
5\end{array}$ & $\begin{array}{l}5 \\
5 \\
5 \\
5\end{array}$ & $\begin{array}{l}5 \\
5 \\
5 \\
5\end{array}$ & $\begin{array}{l}5 \\
5 \\
5 \\
5\end{array}$ \\
\hline & $\begin{array}{c}\text { epoxy } 1 \\
\text { epoxy } 2 \\
\text { polyurcthane } \\
\text { acrylic }\end{array}$ & 35 & $\begin{array}{c}4-5 \\
4 \\
4 \\
4-5\end{array}$ & $\begin{array}{c}4-5 \\
4 \\
4-5 \\
4\end{array}$ & $\begin{array}{l}4 \\
5 \\
5 \\
5\end{array}$ & $\begin{array}{l}5 \\
5 \\
5 \\
5\end{array}$ & $\begin{array}{r}5 \\
\cdot 5 \\
5 \\
5\end{array}$ & $\begin{array}{l}5 \\
5 \\
5 \\
5\end{array}$ \\
\hline & $\begin{array}{c}\text { epoxy } 1 \\
\text { epoxy } 2 \\
\text { polyurethane } \\
\text { acrylic }\end{array}$ & 42 & $\begin{array}{c}4-5 \\
4 \\
4 \\
4-5\end{array}$ & $\begin{array}{c}4-5 \\
4 \\
4-5 \\
4\end{array}$ & $\begin{array}{l}4 \\
5 \\
5 \\
5\end{array}$ & $\begin{array}{l}5 \\
5 \\
5 \\
5\end{array}$ & $\begin{array}{l}5 \\
5 \\
5 \\
5\end{array}$ & $\begin{array}{l}5 \\
5 \\
5 \\
5\end{array}$ \\
\hline \multirow[t]{5}{*}{$\begin{array}{l}\text { Polyester } \\
\text { Fiberglass } \\
\text { Board }\end{array}$} & $\begin{array}{c}\text { epoxy } 1 \\
\text { epoxy } 2 \\
\text { polyurethane } \\
\text { acrylic }\end{array}$ & 0 & $\begin{array}{c}5 \\
4-5 \\
5 \\
4-5\end{array}$ & $\begin{array}{c}5 \\
4-5 \\
5 \\
5\end{array}$ & $\begin{array}{l}5 \\
5 \\
5 \\
5\end{array}$ & $\begin{array}{l}5 \\
5 \\
5 \\
5\end{array}$ & $\begin{array}{l}5 \\
5 \\
5 \\
5\end{array}$ & $\begin{array}{l}5 \\
5 \\
5 \\
5\end{array}$ \\
\hline & $\begin{array}{l}\text { epoxy } 1 \\
\text { epoxy } 2 \\
\text { polyurethane } \\
\text { acrylic }\end{array}$ & 7 & $\begin{array}{c}5 \\
4-5 \\
5 \\
4-5\end{array}$ & $\begin{array}{c}5 \\
4-5 \\
5 \\
5\end{array}$ & $\begin{array}{l}5 \\
5 \\
5 \\
5\end{array}$ & $\begin{array}{l}5 \\
5 \\
5 \\
5\end{array}$ & $\begin{array}{l}5 \\
5 \\
5 \\
5\end{array}$ & $\begin{array}{l}5 \\
5 \\
5 \\
5\end{array}$ \\
\hline & $\begin{array}{l}\text { epoxy } 1 \\
\text { epoxy } 2 \\
\text { polyurethane } \\
\text { acrylic }\end{array}$ & 14 & $\begin{array}{c}4-5 \\
4 \\
4-5 \\
4-5\end{array}$ & $\begin{array}{c}4-5 \\
4 \\
4-5 \\
4\end{array}$ & $\begin{array}{l}5 \\
5 \\
5 \\
5\end{array}$ & $\begin{array}{l}5 \\
5 \\
5 \\
5\end{array}$ & $\begin{array}{l}5 \\
5 \\
5 \\
5\end{array}$ & $\begin{array}{l}5 \\
5 \\
5 \\
5\end{array}$ \\
\hline & $\begin{array}{c}\text { epoxy } 1 \\
\text { epoxy } 2 \\
\text { polyurcthanc } \\
\text { acrylic }\end{array}$ & 21 & $\begin{array}{l}4-5 \\
4 \\
4-5 \\
4-5\end{array}$ & $\begin{array}{c}4-5 \\
4 \\
4-5 \\
4\end{array}$ & $\begin{array}{l}5 \\
5 \\
5 \\
5\end{array}$ & $\begin{array}{l}5 \\
5 \\
5 \\
5\end{array}$ & $\begin{array}{l}5 \\
5 \\
5 \\
5\end{array}$ & $\begin{array}{l}5 \\
5 \\
5 \\
5\end{array}$ \\
\hline & $\begin{array}{c}\text { epoxy } 1 \\
\text { epoxy } 2 \\
\text { polyurethane } \\
\text { acrylic }\end{array}$ & 28 & $\begin{array}{c}4-5 \\
4 \\
4-5 \\
4-5\end{array}$ & $\begin{array}{c}4-5 \\
4 \\
4-5 \\
4\end{array}$ & $\begin{array}{l}5 \\
5 \\
5 \\
5\end{array}$ & $\begin{array}{l}5 \\
5 \\
5 \\
5\end{array}$ & $\begin{array}{l}5 \\
5 \\
5 \\
5\end{array}$ & $\begin{array}{l}5 \\
5 \\
5 \\
5\end{array}$ \\
\hline
\end{tabular}

a See Table 3.8 for a description of the numerical ranking code for assessing tamper tapes. 
Table 4.11. (Continued)

\begin{tabular}{|c|c|c|c|c|c|c|c|c|}
\hline \multirow[b]{3}{*}{ Surface } & \multirow[b]{3}{*}{ Adhesive } & \multirow{3}{*}{$\begin{array}{c}- \\
\text { Days of } \\
\text { Exposure }\end{array}$} & \multicolumn{6}{|c|}{ Condition of Tamper Tape ${ }^{\mathbf{a}}$} \\
\hline & & & \multicolumn{2}{|c|}{ Appearance } & \multicolumn{2}{|c|}{ Security Emblem } & \multicolumn{2}{|c|}{ Surface Adhesion } \\
\hline & & & $\begin{array}{c}\text { Window } \\
\text { Area }\end{array}$ & $\begin{array}{l}\text { Vinyl } \\
\text { Area } \\
\end{array}$ & $\begin{array}{c}\text { Window } \\
\text { Area }\end{array}$ & $\begin{array}{l}\text { Vinyl } \\
\text { Area } \\
\end{array}$ & $\begin{array}{c}\text { Window } \\
\text { Area }\end{array}$ & Overall \\
\hline \multirow[t]{2}{*}{$\begin{array}{l}\text { Polyester } \\
\text { Fiberglass } \\
\text { Board } \\
\text { (Cont.) }\end{array}$} & $\begin{array}{c}\text { epoxy } 1 \\
\text { epoxy } 2 \\
\text { polyurethane } \\
\text { acrylic }\end{array}$ & 35 & $\begin{array}{c}4-5 \\
4 \\
4 \\
4-5\end{array}$ & $\begin{array}{c}4-5 \\
4 \\
4-5 \\
4\end{array}$ & $\begin{array}{l}5 \\
5 \\
5 \\
5\end{array}$ & $\begin{array}{l}5 \\
5 \\
5 \\
5\end{array}$ & $\begin{array}{l}5 \\
5 \\
5 \\
5\end{array}$ & $\begin{array}{l}5 \\
5 \\
5 \\
5\end{array}$ \\
\hline & $\begin{array}{c}\text { epoxy } 1 \\
\text { epoxy } 2 \\
\text { polyurethane } \\
\text { acrylic }\end{array}$ & 42 & $\begin{array}{c}4-5 \\
4 \\
4 \\
4-5\end{array}$ & $\begin{array}{c}4-5 \\
4 \\
4-5 \\
4\end{array}$ & $\begin{array}{l}5 \\
5 \\
5 \\
5\end{array}$ & $\begin{array}{l}5 \\
5 \\
5 \\
5\end{array}$ & $\begin{array}{l}5 \\
5 \\
5 \\
5\end{array}$ & $\begin{array}{l}5 \\
5 \\
5 \\
5\end{array}$ \\
\hline \multirow[t]{7}{*}{$\begin{array}{l}\text { Mil. Spec. } \\
\text { PUb } \\
\text { Painted } \\
\text { Steel }\end{array}$} & $\begin{array}{c}\text { epoxy } 1 \\
\text { epoxy } 2 \\
\text { polyurethane } \\
\text { acrylic }\end{array}$ & 0 & $\begin{array}{l}5 \\
5 \\
5 \\
5\end{array}$ & $\begin{array}{l}5 \\
5 \\
5 \\
5\end{array}$ & $\begin{array}{l}5 \\
5 \\
5 \\
5\end{array}$ & $\begin{array}{l}5 \\
5 \\
5 \\
5\end{array}$ & $\begin{array}{l}5 \\
5 \\
5 \\
5\end{array}$ & $\begin{array}{l}5 \\
5 \\
5 \\
5\end{array}$ \\
\hline & $\begin{array}{c}\text { epoxy } 1 \\
\text { epoxy } 2 \\
\text { polyurethane } \\
\text { acrylic }\end{array}$ & 7 & $\begin{array}{l}5 \\
5 \\
5 \\
5\end{array}$ & $\begin{array}{l}5 \\
5 \\
5 \\
5\end{array}$ & $\begin{array}{l}5 \\
5 \\
5 \\
5\end{array}$ & $\begin{array}{l}5 \\
5 \\
5 \\
5\end{array}$ & $\begin{array}{l}5 \\
5 \\
5 \\
5\end{array}$ & $\begin{array}{l}5 \\
5 \\
5 \\
5\end{array}$ \\
\hline & $\begin{array}{c}\text { epoxy } 1 \\
\text { epoxy } 2 \\
\text { polyurethane } \\
\text { acrylic }\end{array}$ & 14 & $\begin{array}{l}4-5 \\
4-5 \\
4-5 \\
4-5\end{array}$ & $\begin{array}{c}4-5 \\
4-5 \\
4-5 \\
4\end{array}$ & $\begin{array}{l}5 \\
5 \\
5 \\
5\end{array}$ & $\begin{array}{l}5 \\
5 \\
5 \\
5\end{array}$ & $\begin{array}{l}5 \\
5 \\
5 \\
5\end{array}$ & $\begin{array}{l}5 \\
5 \\
5 \\
5\end{array}$ \\
\hline & $\begin{array}{c}\text { epoxy } 1 \\
\text { epoxy } 2 \\
\text { polyurethane } \\
\text { acrylic }\end{array}$ & 21 & $\begin{array}{l}4-5 \\
4-5 \\
4-5 \\
4-5\end{array}$ & $\begin{array}{c}4-5 \\
4-5 \\
4-5 \\
4\end{array}$ & $\begin{array}{l}5 \\
5 \\
5 \\
5\end{array}$ & $\begin{array}{l}5 \\
5 \\
5 \\
5\end{array}$ & $\begin{array}{l}5 \\
5 \\
5 \\
5\end{array}$ & $\begin{array}{l}5 \\
5 \\
5 \\
5\end{array}$ \\
\hline & $\begin{array}{c}\text { epoxy } 1 \\
\text { epoxy } 2 \\
\text { polyurethane } \\
\text { acrylic }\end{array}$ & 28 & $\begin{array}{l}4-5 \\
4-5 \\
4-5 \\
4-5\end{array}$ & $\begin{array}{c}4-5 \\
4-5 \\
4-5 \\
4\end{array}$ & $\begin{array}{l}4 \\
5 \\
5 \\
5\end{array}$ & $\begin{array}{l}5 \\
5 \\
5 \\
5\end{array}$ & $\begin{array}{l}5 \\
5 \\
5 \\
5\end{array}$ & $\begin{array}{l}5 \\
5 \\
5 \\
5\end{array}$ \\
\hline & $\begin{array}{c}\text { epoxy } 1 \\
\text { epoxy } 2 \\
\text { polyurethane } \\
\text { acrylic }\end{array}$ & 35 & $\begin{array}{c}4-5 \\
4-5 \\
4 \\
4-5\end{array}$ & $\begin{array}{l}4-5 \\
4-5 \\
4-5 \\
4-5\end{array}$ & $\begin{array}{l}5 \\
5 \\
5 \\
5\end{array}$ & $\begin{array}{l}5 \\
5 \\
5 \\
5\end{array}$ & $\begin{array}{l}5 \\
5 \\
5 \\
5\end{array}$ & $\begin{array}{l}5 \\
5 \\
5 \\
5\end{array}$ \\
\hline & $\begin{array}{c}\text { epoxy } 1 \\
\text { epoxy } 2 \\
\text { polyurethane } \\
\text { acrylic }\end{array}$ & 42 & $\begin{array}{l}4-5 \\
4-5 \\
4 \\
4-5\end{array}$ & $\begin{array}{c}4-5 \\
4-5 \\
4-5 \\
4\end{array}$ & $\begin{array}{l}5 \\
5 \\
5 \\
5\end{array}$ & $\begin{array}{l}5 \\
5 \\
5 \\
5\end{array}$ & $\begin{array}{l}5 \\
5 \\
5 \\
5\end{array}$ & $\begin{array}{l}5 \\
5 \\
5 \\
5\end{array}$ \\
\hline
\end{tabular}

a See Table 3.8 for a description of the numerical ranking code for assessing tamper tapes.

b Polyurethane 


\section{DISTRIBUTION}

No. of

Copies

\section{OFFSITE}

2 DOE/Office of Scientific and

Technical Information

$2 \quad$ Michael O'Connell

Office of Research \& Development/NN-20

U.S. Department of Energy

1000 Independence Ave.

Washington, DC 20585

LTC William McKune

Defense Nuclear Agency/OPAC

6801 Telegraph Road

Alexandria, VA 22310-3398

5 Gordon Pickett

Battelle Columbus

505 King Avenue

Columbus, OH 43201-2693

\section{QNSITE}

21 Pacific Northwest Laboratory

J. R. Abraham, P8-08

J. L. Fuller, K6-48

H. A. Undem, K6-48 (4)

B. W. Wright, P7-07 (8)

C. W. Wright, P7-07

Publishing Coordination

Technical Report Files (5)

Distr. 1 